\title{
SIMULACIÓN ESTOCÁSTICA DE EVENTOS EN REDES DE DISTRIBUCIÓN DE ENERGÍA ELÉCTRICA
}




\section{SIMULACIÓN ESTOCÁSTICA DE EVENTOS EN REDES DE DISTRIBUCIÓN DE ENERGÍA ELÉCTRICA}

por

Fernando A. Frechoso Escudero

presentada en la

UNIVERSIDAD DE VALLADOLID

en el

DEPARTAMENTO DE INGENIERÍA ELÉCTRICA

en la

ESCUELA TÉCNICA SUPERIOR DE INGENIEROS INDUSTRIALES

para la obtención del

Grado de Doctor Ingeniero Industrial 


\title{
SIMULACIÓN ESTOCÁSTICA DE EVENTOS EN REDES DE DISTRIBUCIÓN DE ENERGÍA ELÉCTRICA
}

\author{
por \\ Fernando A. Frechoso Escudero
}

Director de Tesis

Dr. D. José Luis del Álamo y del Sarmiento

\section{TRIBUNAL CALIFICADOR}

\author{
Presidente: Dr.D. Ricardo Álvarez Isasi \\ Catedrático de Universidad. Área de Ingeniería Eléctrica. \\ Escuela Superior de Ingenieros de Bilbao. \\ Universidad del País Vasco. \\ Vocales: $\quad$ Dr.D. Jesús Fraile Mora \\ Catedrático de Universidad. Área de Ingeniería Eléctrica. \\ E.T.S. de Ingenieros de Caminos, Canales y Puertos. \\ Universidad Politécnica de Madrid.

\section{Dr.D. José Cidrás Pidre} \\ Catedrático de Universidad. Área de Ingeniería Eléctrica. \\ E.T.S. de Ingenieros Industriales. \\ Universidad de Vigo.

\section{Dr.D. Francisco Javier Alonso Ripoll} \\ Profesor Titular de Universidad. Área de Ingeniería Eléctrica. \\ E.T.S. de Ingenieros Industriales. \\ Universidad de Valladolid. \\ Secretario: Dr.D. Manuel Vicente Riesco Sanz \\ Profesor Titular de Universidad. Área de Ingeniería Eléctrica. \\ E.T.S. de Ingenieros Industriales. \\ Universidad de Valladolid.
}


A mis padres y hermanos 
Quisiera expresar mi más sincero agradecimiento a todos aquellos que, de alguna manera, tuvisteis que ver con el desarrollo del presente trabajo. Me sería muy difícil enumeraros a todos, pues abundáis y no tendría suficiente con una página. Tampoco quisiera olvidarme de nadie, así que hablando en general, puedo decir que, cada uno, habéis cumplido una pequeña misión durante el tiempo que me ha llevado desarrollar esta Tesis. Familia, amigos $y$ compañeros de trabajo, a todos, gracias.

En particular quiero agradecer a $D$. José Luis del Alamo, director de esta Tesis Doctoral, su orientación, dedicación y apoyo en todo momento. A todos los componentes del Proyecto CALSER, la gran ayuda prestada, gracias a la cual se ha cimentado este trabajo. $Y$, en fin, corriendo el riesgo de repetirme, a todos aquellos que con su cariño me rodearon y no apretaron demasiado, gracias. 
"Alea jacta est" Julio Cesar 


\section{O. INTRODUCCIÓN}

0.1 Objetivos. 1

0.2 Planteamiento. Justificación. 3

0.3 Antecedentes. 5

\section{FORMULACIÓN MATEMÁTICA}

1.0 Conceptos de Fiabilidad.

1.0.1 Fiabilidad. 11

1.0.2 Disponibilidad. $\quad 17$

1.0.3. Fiabilidad y Disponibilidad de Conjuntos de Componentes en Serie. 18

1.0.4. Índices de Calidad de Servicio. 19

1.1 Grupos Mínimos de Corte. 22

1.1.1 Redes de Fiabilidad. $\quad 22$

1.1.2 Análisis de Redes. Método de los Grupos Mínimos de Corte. 25

1.2 Simulación. Método de Montecarlo. 27

\section{MODELO PROPUESTO}

2.0 Generación de Variables Aleatorias Uniformes. 29

2.1 Pruebas sobre los Generadores de Variables Aleatorias Uniformes. $\quad 33$

2.2 Generación de Variables Aleatorias No-uniformes. 39

2.3 Diseño del Experimento de Simulación. 42

2.3.1 Sin considerar la influencia de las condiciones meteorológicas. $\quad 42$

2.3.2 Considerando la influencia de las condiciones meteorológicas. $\quad 44$

2.4 Simulación de Eventos en la Red. 47

2.5 La Herramienta Informática. 56

\section{ESCENARIOS DE APLICACIÓN}

3.0 Introducción. 61

3.1 Redes de Ensayo. 63

3.1.1 Escenarios para la obtención de Índices de Calidad. 67

3.1.2 Escenarios para el análisis del comportamiento de las Distribuciones. 70 


\section{RESULTADOS}

4.0 Generadores de Variables Aleatorias. Resultados de las Pruebas

Estadísticas.

4.1 Diseño de Experimentos. Resultados. 75

4.1.1 Sin considerar la influencia de las condiciones meteorológicas. $\quad 75$

4.1.2 Considerando la influencia de las condiciones meteorológicas. $\quad 77$

4.2 Índices de Calidad. Resultados. 79

4.3 Distribuciones y su comportamiento. 98

4.4 Simulación del comportamiento de la Red mediante las distribuciones 136

asociadas a sus puntos de carga.

\section{CONCLUSIONES}

5.0 Conclusiones

BIBLIOGRAFÍA

\section{ANEXOS}

Anexo 1: Componentes Considerados.

Anexo 2: Parámetros de Fiabilidad de los Componentes.

Anexo 3: Datos Topológicos de las Redes de Ensayo. 


\subsection{OBJETIVO S}

Si se realiza un análisis estadístico de las Tasas de Indisponibilidad de Servicio a usuarios, se encuentra con facilidad que dentro del Sistema Eléctrico, es la Distribución de Energía Eléctrica el que aporta una mayor contribución. De hecho, del orden del $80 \%$ de las interrupciones de servicio son resultados de incidentes en el Sistema de Distribución. Por otro lado, es evidente la creciente exigencia en lo que se refiere a Calidad del Suministro de Energía Eléctrica como Producto. Se concluye entonces, que es importante disponer de un procedimiento de análisis de los eventos que se producen en una Red de Distribución Eléctrica en Media Tensión, que facilite la toma de decisiones sobre la implantación de nuevas instalaciones, modificación y crecimiento de estructuras ya existentes, y establecimiento de pautas de mantenimiento correctivo y preventivo.

Esta Tesis se enmarca dentro del Proyecto CALSER (Análisis Predictivo de la Fiabilidad en Redes de Distribución Para la mejora de la Calidad de Servicio), que el Departamento de Ingeniería Eléctrica de la Universidad de Valladolid está realizando en colaboración con Iberdrola y subvencionado por OCIDE dentro de los Proyectos de Investigación Electrotécnico (PIE), con el número 132.319. Los índices de calidad al uso en Sistemas de Distribución, obtenidos como resultado de métodos analíticos tradicionales, como el de Frecuencia-Duración y el basado en Procesos homogéneos de Markov, ha sido necesario validarlos con un método como el de Simulación Estocástica de Eventos por la ventaja que presentaba, de poder incorporar un tratamiento más realista de la explotación de un Sistema de Distribución y la 
facilidad de poder suministrar nuevos índices de acotado de la calidad basados en las distribuciones de probabilidad del "Tiempo hasta la Interrupción" y del "Tiempo de Indisponibilidad" que se obtienen para cada punto de carga de la red bajo análisis.

El objetivo fundamental que se plantea para esta Tesis es el diseño de un procedimiento de simulación estocástica de eventos para diversas configuraciones de componentes en una Red de Distribución Eléctrica de Media Tensión. Este procedimiento de simulación, implementado en computadora digital, partiendo de los datos estimados de tasas de fallo y tiempos de reposición de servicio de los componentes de la red, deberá permitir, por un lado verificar o, en su caso, predecir los índices de calidad al uso en Sistemas de Distribución y, por otro, obtener la distribución de frecuencias del "Tiempo hasta la Interrupción" y del "Tiempo de Reposición de Servicio" o "Tiempo de Indisponibilidad" de cada punto de carga del Sistema de Distribución de Energía Eléctrica en Media Tensión, como magnitudes debidamente parametrizadas.

La orientación del método de análisis hacia la predicción de índices de calidad, esto es, la determinación a priori de tales índices, constituirá una potente herramienta, ya sea como ayuda a la toma de decisiones sobre actuaciones a fin de mejorar la calidad de servicio, ya sea como análisis de la bondad de datos utilizados o estrategias de explotación empleadas, toda vez que, aquéllos son determinados en la actualidad, por las compañías eléctricas, a posteriori, a partir de incidencias registradas durante largos períodos de tiempo.

A partir de la distribución de frecuencias del "Tiempo hasta la Interrupción" y del "Tiempo de Indisponibilidad" de cada punto de carga de la red, encontraremos los límites inferior y superior de la duración de las interrupciones y de la continuidad de servicio, como nuevos índices que nos permiten hablar asimismo de la calidad del servicio que ofrece una Compañía Eléctrica.

La capacidad de ajustar por una distribución estadística conocida las distribuciones de frecuencias del "Tiempo hasta la Interrupción" y del "Tiempo de Indisponibilidad" de cada punto de carga de la red, facilitará la búsqueda de otros índices asociados con una distribución de probabilidad, como la probabilidad de que la duración de una interrupción sea menor que una de valor dado o la probabilidad de que se llegue a un tiempo dado sin que el servicio se halla interrumpido.

La utilización de estas dos distribuciones asociadas a cada punto de carga para posteriores simulaciones, como si de la simulación de un único elemento se tratase, incrementaría la velocidad de cómputo en la búsqueda de otros resultados derivados de los ciclos de funcionamiento-fallo de los puntos carga, y asociados, por ejemplo, a los costos que los fallos de la red provocan en el consumidor o la compañía.

Dado que los métodos de simulación, habilitan técnicas de análisis más flexibles que los planteamientos denominados clásicos, esta Tesis se centrará en sistemas cuyos componentes se encuentren sometidos a ciclos de funcionamientoreparación bajo condiciones atmosféricas, en su caso, variables, y en donde los 
tiempos correspondientes, no se hallen, necesariamente, distribuidos de forma exponencial.

Cuantitativamente, mostraremos, en función de diferentes factores, el grado de aproximación de la solución aportada. En particular, obtendremos resultados comparativos en aquellos supuestos en que sea aplicable una técnica basada en el empleo de procesos homogéneos de Markov y, en su caso, Grupos Mínimos de Corte. Se estudiará la dependencia que los índices de calidad, al uso en Sistemas de Distribución, tienen respecto las características de las distribuciones que siguen los diferentes tiempos entre eventos en la red.

Analizaremos si, realmente, las aportaciones realizadas mediante la simulación modifican sustancialmente los resultados de las técnicas clásicas y, en consecuencia, el grado de validez de las hipótesis simplificadoras empleadas por tales técnicas.

Finalmente, destacaremos las aportaciones de la simulación, como una mejor aproximación al análisis del funcionamiento real de las Redes de Distribución y a un mayor conocimiento de la fiabilidad de éstas.

\subsection{PLANTEAMIENTO. JUSTIFICACIÓN}

En los últimos años se han acrecentado los esfuerzos en las líneas de investigación conducentes a una mejora en los índices de calidad de los Sistemas Eléctricos de Potencia. El camino recorrido tanto en la parte de generación como en la de transporte es muy importante, encontrándose, sin embargo, la investigación sobre Sistemas de Distribución de Energía Eléctrica, prácticamente en sus inicios. En este campo es, precisamente, donde esta Tesis pretende realizar su contribución.

En esta Tesis optamos por la simulación estocástica como método para realizar nuestro estudio por dos razones principalmente, implícitas ya en las recogidas por Naylor (L_NAY-66):

- En primer lugar, el modelo usado en la técnica analítica es generalmente una simplificación del sistema que, a veces, puede llegar a ser poco realista. La simulación, sin embargo, puede incorporar y simular cualquier característica del sistema que pueda ser reconocida. En nuestro caso existen, principalmente, tres aproximaciones analíticas (método de la tasa media de fallo, método de duración y frecuencia, y el método basado en procesos de Markov), que no resuelven cuestiones como distribuciones temporales de eventos en la red diferentes a la exponencial o la influencia de los fallos producidos simultáneamente (modo común). Con la técnica de simulación estocástica esperamos incorporar estas características a nuestro modelo.

- En segundo lugar, las técnicas de simulación pueden suministrar un amplio campo de parámetros de salida incluyendo todos los momentos y las funciones 
de densidad de probabilidad completas, mientras que la salida de los métodos analíticos está generalmente limitada sólo a valores esperados.

Nuestro esquema de trabajo comenzará dando una sucinta visión sobre la teoría de fiabilidad aplicada a sistemas eléctricos; trataremos, por tanto, de los conceptos de componentes reparables y no reparables, de sus tasas de fallo y tiempos de reposición de servicio, así como de las funciones de distribución de probabilidad de los tiempos asociados a esos componentes e involucrados en procesos de fallo y reposición de servicio.

Después de pasar revista al método de los Grupos Mínimos de Corte, que nos permitirá, de forma sencilla, aplicar las técnicas de simulación a redes de cierta complejidad, comenzaremos la descripción de la técnica de simulación estocástica que empleamos, conocida como método de Montecarlo. Como introducción al mismo, realizaremos una breve disgresión acerca de los generadores de números aleatorios, así como un análisis sobre la bondad de los mismos, toda vez que constituyen un aspecto básico del método de simulación a seguir. Para finalizar, llevaremos a cabo una serie de pruebas de precisión sobre un conjunto de generadores, optimizando exactitud y tiempo de cálculo.

Presentaremos a continuación, tanto la técnica de simulación, como el modelo matemático a desarrollar para las redes consideradas. Este modelo será validado previamente realizando una serie de pruebas con un sencillo sistema. La técnica desarrollada tendrá dos etapas bien diferenciadas.

- Una primera, consistirá en establecer a partir de la red física y de su lógica de funcionamiento, una red de sucesos que, al producirse, provoquen periodos de indisponibilidad para cada punto de carga.

- La segunda etapa, consistirá en aplicar la simulación secuencial (generación de ciclos de operación-fallo a lo largo del tiempo) a cada uno de los eventos que constituyen estas redes de fiabilidad, hallando, para cada punto de carga, una secuencia equivalente por asociación lógica de eventos.

Estas secuencias equivalentes, que representarán el comportamiento simulado a los largo del tiempo, nos permitirán obtener los datos necesarios para el cálculo de los índices de Calidad de Servicio, y las distribuciones de "Tiempo hasta la Interrupción" y del "Tiempo de Indisponibilidad" de cada punto de carga. También, incidiremos a lo largo de ese capítulo en las políticas de mantenimiento, tanto en su aspecto correctivo como preventivo.

Posteriormente describiremos, brevemente, la herramienta informática implementada, su planteamiento y algoritmos, el funcionamiento práctico de la aplicación y finalmente los ficheros de resultados.

Seguidamente haremos una descripción esquemática de las Redes de Distribución de Energía Eléctrica en media tensión, los diferentes tipos de 
estructuras, y las correspondientes filosofías de explotación. Describiremos brevemente los principales componentes de las mismas, así como las protecciones y la lógica de funcionamiento.

Nuestros sistemas a estudio consistirán en Redes de Distribución de Energía Eléctrica en Media Tensión, que dividiremos en aéreas, subterráneas y mixtas. Para cada una de esta redes definiremos unos escenarios, como un conjunto de hipótesis sobre la distribución del tiempo entre eventos y características del experimento de simulación, bajo los que desarrollaremos nuestros experimentos de simulación.

A continuación presentaremos los índices de calidad obtenidos mediante nuestra técnica de simulación para diversas condiciones de operación en las redes empleadas, y los compararemos con los obtenidos mediante el método analítico basado en procesos de Markov. También presentaremos las variaciones que experimentan estos índices frente a modificación de las características de las distribuciones que siguen los tiempos entre eventos en la red.

Seguidamente, analizaremos las variaciones de las distribuciones de frecuencias de "tiempo hasta la interrupción" y del "tiempo de indisponibilidad" de cada punto de carga con las condiciones de operación de las redes utilizadas. Y finalizaremos, ajustando estas distribuciones de frecuencias por distribuciones de probabilidad conocidas.

Finalizaremos esta Tesis con una relación de conclusiones y de las aportaciones que realiza.

\subsection{ANTECEDENTES}

Las primeras aplicaciones prácticas de la teoría de fiabilidad lo fueron en el campo militar y aerospacial (L_VILL-92). Aunque antes de 1964 se conocían publicaciones sobre fiabilidad aplicada a Sistemas Eléctricos, fue a partir de ese año cuando se publicaron dos artículos básicos, a cargo de Todd (A_TOD-64), y Gaver y Montmeat (A_GAV-MON-64), describiendo dos técnicas diferentes aplicadas al calculo de la Fiabilidad en Redes de Transporte de Energía Eléctrica: El Método de la Tasa Media de Fallo y el Método de Frecuencia-Duración, respectivamente.

El método de la Tasa Media de Fallo (A_TOD-64) es sencillo de aplicar a redes complejas y, al igual que la mayoría de los métodos analíticos, está orientado más a proporcionar una medida de la continuidad de servicio que un índice de la calidad del mismo, examinando las condiciones que deben existir para que la "potencia fluya" por combinaciones de elementos serie y paralelo. Los componentes en que se divide la red pueden encontrarse en dos estados, fallo y funcionamiento. La probabilidad de que un componente esté en modo de fallo viene dada por su tasa de fallo que, en este método, es igual al sumatorio de los días en los que, un fallo, ha provocado, al menos, una interrupción significativa, dividido por todo el período de 
días considerado. Se supone que los sucesos de fallo de los componentes son estocásticamente independientes.

Entre los inconvenientes que presenta, destaca el no poder incluir la influencia de las condiciones atmosféricas sobre la red, por lo que sólo resulta un buen método cuando las condiciones atmosféricas no influyen en el estado de operatividad de los componentes de la red. Tampoco permite incluir la influencia de la variación en la carga por lo que los índices de energía no suministrada, calculados, serán valores medios. Y como último inconveniente, supone que los fallos que ocurren un mismo día son simultáneos, por lo que no puede reflejar, por ejemplo, el efecto de los fallos en cascada provocados por una tormenta.

El segundo de estos métodos (A_GAV-MON-64), denominado el método de Frecuencia y Duración predice la frecuencia y duración de las interrupciones de servicio en los puntos de carga de la red suponiendo que las distribuciones de probabilidad de las tasas de fallo y tiempos de reparación de sus componentes están exponencialmente distribuidas. Este método, a diferencia del anterior, introduce la influencia del tiempo atmosférico que condicionará la operatividad del componente.

Este método puede aplicarse suponiendo, para cada elemento, tasas medias anuales de fallo y reparación, o suponiendo tasas de fallo y de reparación medias estacionales, esto es, distintas para cada tipo de tiempo atmosférico que consideremos. El efecto de fallos en cascada cuando hay tormentas puede hacer inexactas las predicciones con tasas medias anuales, siendo más evidente este efecto, en redes con elementos en paralelo. La aplicación del método se complica a medida que la red se hace más compleja, si se utilizan tasas medias estacionales, en vez de anuales. En una artículo muy posterior, Koval y Billinton (A_KOV-BIL-79) emplean este método utilizando tasas medias anuales para estudiar el efecto de los equipos de protección en los índices de calidad de la red.

El método proporciona el número medio de interrupciones, el tiempo medio por interrupción y el tiempo total medio de interrupción, por punto de carga y por año.

Este método, incluye la influencia del mantenimiento preventivo en los índices de calidad de la red. Sin embargo lo hace tratándolo como una variable aleatoria más, cuando en realidad el mantenimiento preventivo lo planifica la empresa eléctrica de antemano y no es, esencialmente, aleatorio.

El empleo de procesos homogéneos de Markov, con Espacio de Estado discreto y Parámetro (tiempo) continuo, para la determinación de las probabilidades de estado estacionario (A_BIL-BOL-68), surgió ante la necesidad de disponer de una herramienta analítica más exacta y que permitiera resolver configuraciones de red complejas en cuanto topología o lógica de funcionamiento. Pero en la práctica, este tratamiento sólo es aplicable a pequeños grupos de componentes ya que las soluciones analíticas de las ecuaciones que se plantean con esta formulación son 
difíciles de obtener, a poco grande que sea la red, y las soluciones numéricas, aunque sencillas de implementar en un ordenador, resultan muy lentas y requieren mucha capacidad de almacenamiento del ordenador. Este método está especialmente indicado para redes con elementos en paralelo, pues en este caso, la solución que aporta es más exacta que los resultados de los precedentes.

$\mathrm{Al}$ igual que en las técnicas anteriores, se admite que la tasa de fallo permanece constante durante toda la vida de los elementos, y que los fallos de éstos son estocásticamente independientes. No es usual, aunque sí posible, considerar la aparición de un segundo fallo mientras la red tiene alguna zona en avería, porque su incorporación supone la aparición de más estados y, en consecuencia, el aumento del tamaño de las matrices estocásticas de transición, sin una contrapartida de una mejora sustancial en la modelización para los sistemas que consideramos.

La implementación en computadora del método de Markov necesita una elevada disponibilidad de memoria incluso para redes de tamaño moderado. Para solventar este problema se recurre a modelos mixtos. En unos se aplica el método de Markov a determinadas zonas de una red para luego agrupar los resultados, como en Ringlee y Goode (A_RIN-GOO-70) donde con un procedimiento del tipo "paso a paso", se estudia la calidad de servicio en los puntos carga de la red. En otros métodos mixtos, se suelen agrupan las líneas en zonas para luego aplicar Markov a éstas como si de elementos individuales se tratasen tal y como, por ejemplo hace Roldán en (A_ROL-ALV-91), en donde se utiliza como herramienta para estudiar la posible reconfiguración de Redes de Distribución a fin de minimizar la energía no suministrada en caso de fallo.

La simulación estocástica, también llamada simulación de Monte Carlo, surgió ante la necesidad de manejar grandes redes y poder introducir de manera flexible un tratamiento más real de la lógica de explotación del conjunto generación-transporte (A_SAL-BIL-85) y no ha sido aplicada, a nuestro conocimiento, en Redes de Distribución de Energía Eléctrica. Esta técnica en lugar de describir directamente el comportamiento global del Sistema, describe su operación en términos de eventos individuales de cada uno de los componentes del mismo por separado (L_SOB-76) (L_COS-82). En particular, es posible dividir el Sistema Eléctrico en elementos cuyo comportamiento se puede predecir, al menos en términos de distribuciones de probabilidad, para cada uno de los diversos estados posibles del Sistema y de sus entradas, siendo posible la incorporación al modelo de las interrelaciones entre los elementos. Una de las principales ventajas de la técnica de la simulación es que podemos utilizar cualquier tipo de distribución de probabilidad para describir ese comportamiento de los elementos y sus interrelaciones. La simulación es, en realidad, una técnica de efectuar experimentos de muestreo sobre el modelo del sistema. Los experimentos se realizan sobre el modelo, en lugar de hacerlo sobre el propio sistema real, ciertamente porque esto último sería irrealizable.

Usualmente se aplica la técnica de simulación a sistemas mixtos, generación y transporte, debido al gran número de variables, relacionadas entre sí, que entran en el 
análisis de la fiabilidad de estos sistemas. El modelo se construye como un conjunto de componentes elementales que, a continuación, se combinan en su orden natural de funcionamiento, teniendo en cuenta sus interacciones mutuas. La acción de simular consiste en registrar el comportamiento conjunto del sistema, a partir de la simulación de un elevado número de eventos individuales en todos y cada uno de los componentes, de tal modo que los experimentos sean virtualmente indistinguibles respecto de los experimentos estadísticos ordinarios.

Dado que en la técnica de la simulación el esfuerzo de cálculo se incrementa cuadráticamente con la exactitud requerida, es necesario acelerar la convergencia y exactitud del muestreo. Para eso se utilizan técnicas de reducción de varianza como el método del "muestreo estratificado o por importancia" (A_CUN-PER-85), o el método que busca un estimador previo mediante técnicas analíticas sencillas (A_OLI-PER-89) (A_PER-PIN-92) (A_PER-PIN-92 bis).

Se debe hacer hincapié en que no es necesario que el modelo de simulación sea una representación completamente realista del sistema real. De hecho, parece que el error en la mayor parte de los modelos de simulación es ser demasiado realistas, en lugar de demasiado idealistas. En aquel caso, el modelo degenera con facilidad en una masa de detalles triviales y tortuosos, de modo que se requiere una gran cantidad de datos y de tiempo de programación y de simulación para obtener una reducida cantidad de información.

En realidad, hay dos técnicas básicas utilizadas en la simulación por Monte Carlo cuando se aplica a la evaluación de la fiabilidad de Sistemas Eléctricos. Se conocen como técnicas secuencial y no-secuencial. La técnica no-secuencial es la más utilizada y la que más bibliografía ha generado y continúa haciéndolo, quizás porque, en general, es más rápida que la secuencial y necesita menos capacidad de almacenamiento. Sólo se limita a muestrear el estado de todos los elementos del sistema y de ahí deduce el estado del sistema de manera no-cronológica (A_BILWEN-91). El principal problema de la simulación no-secuencial es que hay que definir previamente todos los estados del sistema y las transiciones de unos a otros (A_BIL-LI-93).

La simulación secuencial es de aplicación más reciente debida, en gran medida, al progreso en la velocidad y capacidad de almacenamiento de los ordenadores. Consiste en generar los ciclos de vida de los componentes que forman el sistema de forma secuencial para, combinando estos ciclos, conocer en todo momento el estado del sistema.

La simulación secuencial se utiliza cuando el estado de un sistema depende de su pasado, de su evolución histórica, toda vez que las hipótesis necesarias para hacer el sistema independiente de su historia llevan a resultados poco reales. Dentro de la simulación secuencial cabe distinguir dos técnicas diferentes según utilicen un incremento de tiempo fijo o variable (L_HIL-LIE-82). En la primera técnica (A_UVE-ALL-92), con incremento de tiempo fijo, empezando con el sistema en su 
estado inicial, en un instante dado, se avanza el tiempo en una pequeña cantidad fija, lo suficientemente pequeña para que se pueda considerar que entre dos intervalos de tiempo se producen un número muy pequeño de cambios en el sistema y, a continuación, se actualiza el sistema, determinando qué eventos (cambios en el estado de cada componente) ocurrieron en esa unidad de tiempo y cuál es el estado resultante del sistema. En la segunda técnica, con incremento de tiempo variable, se generan los tiempos entre evento y evento (cambios en el estado de cada componente) en el sistema, actualizando su reloj con estos tiempos variables.

Con la simulación secuencial disponemos de dos ventajas añadidas; la primera es que podemos hallar los intervalos de confianza de los índices que encontremos (A_-WAN-89), y la segunda es que podemos hallar, de una manera sencilla, la distribución del tiempo necesario para restaurar el servicio en cada punto de carga después de un fallo y la del tiempo transcurrido entre esas restauraciones y el siguiente fallo, así como sus máximos, lo que nos da una idea, no tanto de la continuidad del suministro, como de su calidad. También podemos hallar distribuciones asociadas a cada uno de los índices de calidad que definamos para cada punto de carga y no sólo su valor esperado como con las otras técnicas (A_PAT-BLA-88). Aunque, como se ha indicado, se precisa más capacidad de almacenamiento y esfuerzo de cálculo que con la técnica no-secuencial, esto puede paliarse, en parte, utilizando métodos de reducción de varianza (A_NOF-PAR-75) (A_SAN-BIL-95).

Una pregunta, que puede surgir cuando se están eligiendo las distribuciones que siguen los tiempos entre eventos en el modelo, es si deben emplearse distribuciones de frecuencias de datos históricos, o bien, buscar la distribución de probabilidad teórica que se ajuste mejor a estos datos. En general, es preferible esta última alternativa, porque estaría más cercana a la predicción del rendimiento futuro esperado, que la reproducción de idiosincrasias de un cierto período del pasado.

Dado que existen redes de distribución aéreas y mixtas con componentes a la intemperie, es extremadamente importante tener en cuenta la influencia del tiempo atmosférico en el comportamiento del sistema, toda vez que su no consideración nos llevaría a unas predicciones optimistas en extremo. Este aspecto ya fue puesto de manifiesto desde los primeros artículos que aplicaban el método de Frecuencia y Duración y el de Markov, donde se incorporaba la influencia del tiempo atmosférico y se estimaba como exponencial la distribución temporal de las duraciones de los ciclos de tiempo atmosférico. Ciertamente al considerar el mismo tiempo atmosférico sobre toda la red, resultaba una sobrestimación de los efectos del mal tiempo sobre la generación de incidentes. Posteriormente y utilizando técnicas mixtas, es posible subdividir la Red en agrupaciones más o menos independientes, y considerar para cada una de ellas condiciones atmosféricas homogéneas. En 1991, Billinton y Wenyuan (A_BIL-WEN-91 bis), buscando un tratamiento más real de la influencia de tiempo atmosférico, introdujeron la distinción entre zonas, con unas características atmosféricas homogéneas (típicamente asociables a zonas geográficas), y líneas; esto permite la consideración de líneas sometidas a diferente tiempo atmosférico en 
alguna de sus partes. Al aplicar una técnica de simulación, es posible asignar distribuciones distintas de la exponencial a los ciclos de tiempo atmosférico, e incluso utilizar históricos para su aproximación.

Ciertamente, estas aproximaciones no tienen en cuenta la naturaleza dinámica de los fenómenos atmosféricos y consideran que los ciclos de tiempo atmosférico son constantes en sus características y afectan al mismo área del sistema y en la misma posición durante toda la duración de un ciclo de simulación. Recientemente, Bhuiyan y Allan (A_BHU-ALL-94) intentan hacer una aproximación más realista, aplicando la simulación secuencial al problema de la influencia atmosférica en los índices de calidad de la red. Aunque no es el propósito de esta Tesis la descripción pormenorizada de los efectos del tiempo atmosférico en una Red de Distribución, si sería posible, en un futuro, introducir en nuestra simulación un modelo atmosférico de este tipo.

Existen otras causas que pueden afectar a la fiabilidad de una Red de Distribución, y que a nivel local pueden tener gran importancia, como son los fallos producidos por animales, caidas de árboles, etc. (A_CHO-TAY-93). Estas causas no serán, tampoco, consideradas en la realización de esta Tesis, aunque, podrían ser modelizadas en un futuro.

El interés por el estudio de la fiabilidad en Redes de Distribución ha sido posterior al de Generación y al de Generación-Transporte siendo, en consecuencia, más reducida la bibliografía específica existente. Cabe, sin embargo citar, de forma complementaria, como aportaciones vinculadas al estudio de fiabilidad en Redes de Distribución las de (A_BIL-GRO-75) que utiliza un modelo atmosférico de tres estados y un algoritmo del tipo Frecuencia y Duración, considerando los fallos temporales, los permanentes, los debidos al mantenimiento y a sobrecargas, y (A_ALL-DIA-79) que focaliza su atención en la reconfiguración de la red en caso de fallo y utiliza una mezcla de Grupos Mínimos de Corte con el método de Markov. Estos autores se limitan al cálculo de los índices básicos de calidad, sin considerar índices, importantes desde el punto de vista de la calidad del servicio al consumidor, relacionados con la duración límite de una interrupción, o con el funcionamiento ininterrumpido, por ejemplo. 


\section{FORMULACIÓN MATEMÁTICA}

\subsection{CONCEPTOS DE FIABILIDAD}

Pasaremos revista, brevemente, a algunos de los modelos matemáticos utilizados en estudios de fiabilidad y disponibilidad de un componente o de un sistema, y su aplicación a redes de distribución de energía eléctrica.

Como aspecto importante, destacaremos la diferencia entre dos conceptos relacionados y que a menudo, por imprecisión del lenguaje, se confunden, o al menos se mezclan: fiabilidad y disponibilidad. Ambas representan una probabilidad; sin embargo, mientras que la fiabilidad es un concepto asociado a componentes no reparables y constituye la probabilidad de que un elemento supere un determinado período de funcionamiento $\sin$ averiarse, la disponibilidad se relaciona con componentes reparables y representa la probabilidad de encontrar operativo a dicho elemento en un determinado instante de tiempo $t$.

Definiremos los Índices de Calidad que nos permitirán evaluar la calidad del servicio en los escenarios de aplicación de esta Tesis.

\subsubsection{Fiabilidad}

Supongamos que tenemos un conjunto de componentes (L_COX-62) (L_BILL-ALL-83), cada uno de los cuales se caracteriza por una variable aleatoria 
no-negativa, $T$, que llamaremos tiempo hasta el fallo que representa el tiempo que tarda el componente en fallar.

Consideraremos que nuestra variable aleatoria no-negativa, $T$, posee una distribución de probabilidad absolutamente continua sobre el rango de tiempo $(0, \infty)$ que vendrá determinada por una función de densidad de probabilidad, $f(t)$.

Suponemos que la variable aleatoria $T$ tiene una $f(t)$,que llamaremos función densidad de fallo, que es cero para valores negativos de $T$ y que cumplirá como cualquier otra función de densidad probabilística:

$$
\begin{gathered}
f(t)=\lim _{\Delta t \rightarrow 0} \frac{\operatorname{prob}(t<T \leq t+\Delta t)}{\Delta t} \\
\operatorname{con} \quad \int_{0}^{\infty} f(t) d t=1
\end{gathered}
$$

Por supuesto, los tiempos hasta el fallo $T_{1}, T_{2}, \ldots$ de los diferentes componentes les supondremos mutuamente independientes.

La distribución de probabilidad de $T$ estará determinada por su $f(t)$ pero, para algunos propósitos, será conveniente trabajar con otras funciones equivalentes a $f(t)$. Una de éstas es la función de distribución, $F(t)$, que proporciona la probabilidad de que un componente halla fallado cuando hemos alcanzado el tiempo $t$. Esto es

$$
F(t)=\operatorname{prob}(T \leq t)=\int_{0}^{t} f(u) d u
$$

Claramente $F(t)$ es una función no-decreciente de t, con $F(0)=0$ y $F(\infty)=1$. La ecuación anterior nos da $F(t)$ en función de su $f(t)$. Al contrario, si derivamos esa ecuación tendremos que $f(t)=d F(t) / d t$, determinando $f(t)$ para una $F(t)$ dada.

Para algunos propósitos es mejor trabajar con la función complementaria de $F(t)$, a la que denominaremos fiabilidad, $R(t)$.

$$
R(t)=\operatorname{prob}(T>t)=1-F(t)=\int_{t}^{\infty} f(u) d u
$$

que da la probabilidad de que un componente no haya fallado llegando a un tiempo $t$. Claramente $R(t)$ es una función no-creciente de t, con $R(0)=1$ y $R(\infty)=0$. También podemos ver, derivando la ecuación anterior, que $f(t)=-d R(t) / d t$.

La relación geométrica entre la función densidad de fallo y la fiabilidad es tal y como se representa en la figura 1.1, donde queda patente que la fiabilidad es una función de distribución que nos da el área bajo la función densidad de fallo a partir de un instante cualquiera de tiempo $t$. 


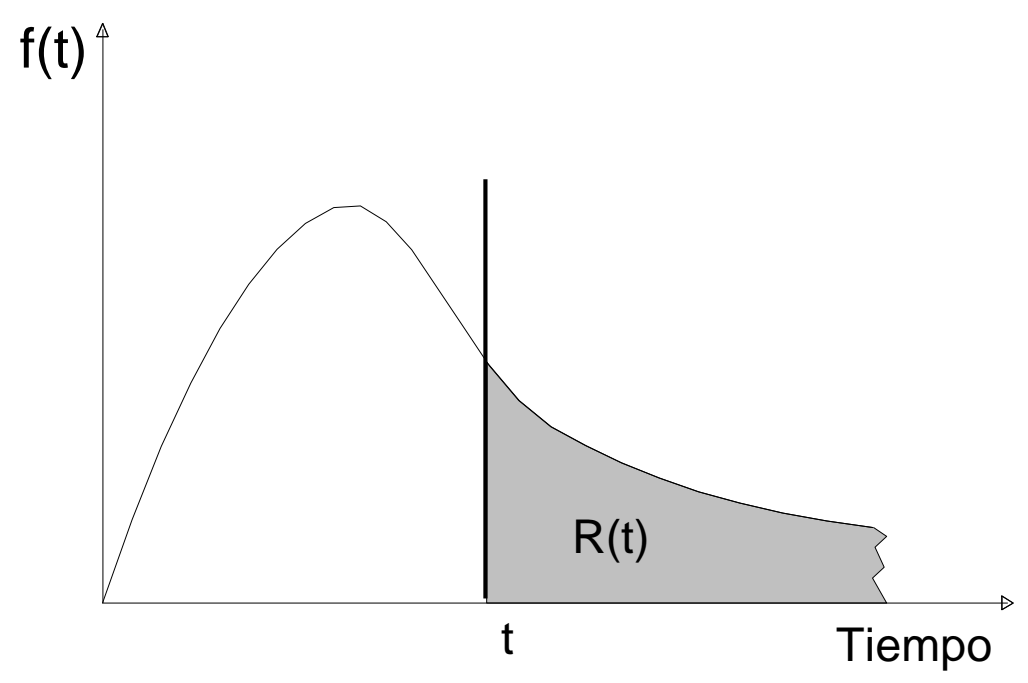

Fig.1.1.-Relación entre la función densidad de fallo y la fiabilidad.

En el análisis de disponibilidad de sistemas y de calidad de servicio, no utilizaremos directamente las funciones de fiabilidad descritas, si no de forma indirecta, a través de otra función equivalente a $f(t)$, que es la tasa de fallo instantánea, $\lambda(t)$, definida como sigue.

$$
\lambda(t)=\lim _{\Delta t \rightarrow 0} \frac{\operatorname{prob}(t<T \leq t+\Delta t / t<T)}{\Delta t}
$$

Donde el numerador representa la probabilidad de que se produzca un fallo durante el intervalo $\Delta t$, condicionada a que en $t$ el componente se encontrase en estado de operación. El denominador permite expresar, en una base de tiempos unitarios, la probabilidad condicionada representada en el numerador. Por tanto, $\lambda(t)$ nos da la probabilidad de fallo inmediato conocida la edad del componente $t$; esta función es la más utilizada hoy en día.

Ahora, si empleamos la fórmula de la probabilidad condicionada de dos eventos, para desarrollar el numerador de la ecuación anterior tenemos que:

$$
\operatorname{prob}(t<T \leq t+\Delta t / t<T)=\frac{\operatorname{prob}(t<T \leq t+\Delta t \cap t<T)}{\operatorname{prob}(t<T)}=\frac{\operatorname{prob}(t<T \leq t+\Delta t)}{\operatorname{prob}(t<T)}
$$

por lo tanto la tasa de fallo queda

$$
\lambda(t)=\lim _{\Delta t \rightarrow 0} \frac{\operatorname{prob}(t<T \leq t+\Delta t)}{\Delta t} \quad \frac{1}{\operatorname{prob}(t<T)}=\frac{f(t)}{R(t)}
$$

que podemos escribir como: 


$$
\lambda(t)=-\frac{1}{R(t)} \frac{d R(t)}{d t}=-\frac{d}{d t}(\log R(t))
$$

usando la condición $R(0)=1$ e integrando en la ecuación anterior tenemos que:

$$
R(t)=\exp \left[-\int_{0}^{t} \lambda(u) d u\right]
$$

y de esta:

$$
f(t)=\lambda(t) \exp \left[-\int_{0}^{t} \lambda(u) d u\right]
$$

Así vemos que una $\lambda(t)$ determina una única $f(t)$.

En el caso particular de que la variable aleatoria $T$ siga una distribución exponencial negativa con una $f(t)=\lambda e^{-\lambda t}$, tenemos que

$$
R(t)=\int_{t}^{\infty} \lambda e^{-\lambda u} d u=e^{-\lambda t}
$$

y por tanto

$$
\lambda(t)=\lambda(\text { constante })
$$

ya que las distribuciones exponenciales se caracterizan por una tasa de fallo constante, la probabilidad de fallo inmediato del componente no depende de su edad.

\section{Elementos de Funcionamiento Discreto}

Hasta ahora nos hemos referido a dispositivos, reparables o no, que funcionan de forma continua en el tiempo. Sin embargo, existen componentes como los interruptores, cuya probabilidad de fallo depende más del número de veces que sean accionados que del tiempo de funcionamiento continuo en su posición de cerrado. Esto se debe a los esfuerzos electrodinámicos a los que se ven sometidos cuando se actúa sobre ellos, especialmente en condiciones de defecto.

Así, dada, una variable aleatoria discreta $c$, definida como el número de ciclos de accionamiento hasta el fallo, la función densidad de fallo a la que hemos aludido, debería ser una función de probabilidad discreta, $p_{n}$ :

$$
p_{n}=p(c=n)
$$

y se definiría como la probabilidad de que la variable aleatoria $c$ valga $n$, es decir de que el fallo sobrevenga al enésimo ciclo de actuación. 
A partir de aquí, podemos deducir las expresiones de fiabilidad e infiabilidad, así como de la tasa de fallo:

$$
\begin{aligned}
& R(n)=P(c>n)=\sum_{i=n+1}^{\infty} p_{i} \\
& \lambda(n)=\frac{p_{n}}{R(n-1)}=\frac{p_{n}}{\sum_{i=n}^{\infty} p_{i}}
\end{aligned}
$$

Cuando existan elementos de funcionamiento discreto en sistemas continuos, se transformarán las variables aleatorias discretas fiabilidad y tasa de fallo, a variables continuas, por ejemplo, considerando el número medio de ciclos de operación por unidad de tiempo.

\section{Curva Temporal de la Tasa de Fallo}

Para describir la variación temporal de la función densidad de fallo de un componente eléctrico sometido a funcionamiento continuado, se suelen distinguir tres etapas claramente diferenciadas (figura 1.2):

- Período de depuración o período infantil.

- Vida útil

- Período de fatiga

Durante cada uno de los tres periodos considerados, la función densidad de fallo del componente se puede ajustar por una distribución de Weibull, sin más que modificar su parámetro de forma:

$$
f(t)=\frac{\beta}{\delta} \cdot\left(\frac{t}{\delta}\right)^{\beta-1} \cdot e^{-\left(\frac{t}{\delta}\right)^{\beta}}
$$

donde $\quad \beta>0$ es el parámetro de forma

y $\quad \delta>0$ es el parámetro de escala

- Período de depuración. La mayor parte de fallos registrados durante este período, corresponden a defectos de fabricación o de diseño, y pueden ser eliminados practicando un control de calidad apropiado. Tan solo una pequeña parte de los fallos son de carácter aleatorio. Tanto la función densidad de fallo como la tasa de fallo tienen un carácter fuertemente decreciente en el tiempo ya que la proporción de elementos con defectos iniciales disminuye cuando estos van fallando, como se aprecia en la figura 1.2 (L_BIL-ALL-92). Para adecuarse al comportamiento señalado, el parámetro de forma $\beta$, de la distribución de Weibull, ha de ser menor que 1. 
$f(t)$

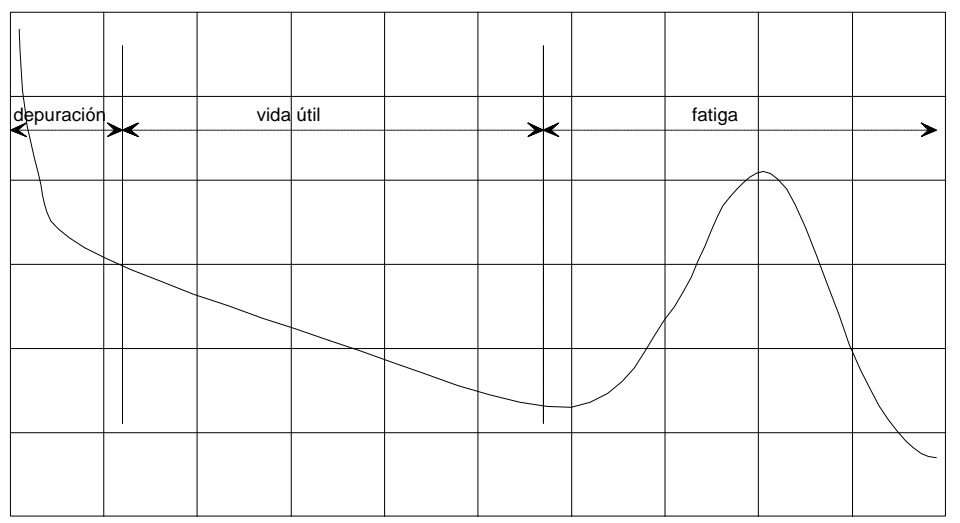

tiempo

$\lambda(\mathrm{t})$

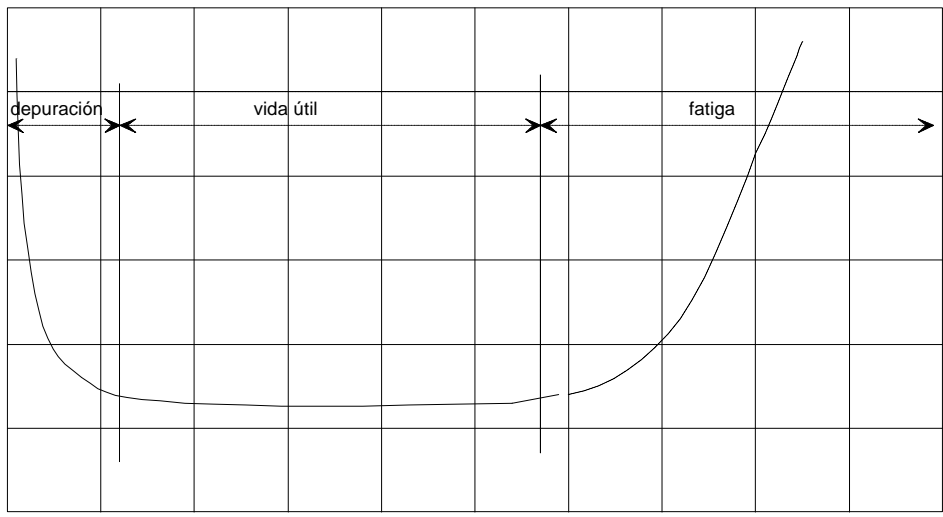

tiempo

Fig.1.2.-Curvas temporales de la función densidad de fallo y de la tasa de fallo

- Vida útil. Corresponde al período en el que los fallos tienen un carácter puramente aleatorio. Durante esta etapa, la tasa de fallo se mantiene sensiblemente constante. Por tanto, el parámetro de forma $\beta$, ha de ser igual a 1 , y la función densidad de fallo corresponderá a una exponencial negativa, cuyo parámetro es la tasa de fallo constante $\lambda$ :

$$
f(t)=\lambda \cdot e^{-\lambda t}
$$

- Período de fatiga. El origen de los fallos durante esta etapa debe buscarse en el envejecimiento del componente. La tasa de fallo crece rápidamente y la función densidad de fallo se aleja ostensiblemente del comportamiento exponencial, como podemos ver en la figura 1.2. El parámetro de forma $\beta$, será mayor que 1 , y la función densidad de fallo corresponderá a una distribución de Rayleigh o una distribución de Gauss. 
Este período puede evitarse practicando una política correcta de mantenimiento preventivo y sustituyendo el componente por otro nuevo cuando éste comience a envejecer.

\section{Características de la Función de Distribución Exponencial Negativa}

En los análisis de fiabilidad de los sistemas eléctricos que llevemos a cabo supondremos, generalmente, que nos hallamos en la vida útil de los componentes y que, por tanto, el tiempo hasta el fallo sigue una distribución de probabilidad exponencial negativa. Será interesante, por tanto, comentar algunas características de dicha función.

Una de las propiedades más interesantes de la distribución de probabilidad exponencial es la carencia de memoria. Este hecho supone, que mientras el componente se encuentre en la etapa de vida útil, la probabilidad de fallo no depende del período previo de funcionamiento. Esto es consecuencia directa del carácter constante de la tasa de fallo.

Los parámetros esperanza y desviación típica correspondientes a la distribución exponencial negativa, son:

$$
\begin{aligned}
& E=\int_{0}^{\infty} t \cdot f(t) d t=\int_{0}^{\infty} t \cdot \lambda e^{-\lambda t} d t=1 / \lambda \\
& \sigma^{2}=\int_{0}^{\infty}(1-E)^{2} \cdot f(t) d t=1 / \lambda^{2}
\end{aligned}
$$

\subsubsection{Disponibilidad}

Como ya introdujimos al comienzo del presente capítulo, cuando hablamos de fiabilidad en componentes reparables, nos estamos refiriendo en realidad al concepto de disponibilidad, es decir, a la probabilidad de encontrar a un componente en funcionamiento en un determinado instante de tiempo. Esta probabilidad podrá ser, bien un valor medio, o bien una función dependiente del tiempo, fruto de una formulación matemática. En nuestro caso, será tratada como un valor medio, deducible directamente a partir de las secuencia de ciclos operación-fallo de un sistema obtenidos mediante simulación estocástica secuencial.

\section{Valores Medios de la Disponibilidad e Indisponibilidad}

Supongamos que el comportamiento de un componente (bien real, o bien simulado) corresponde a una secuencia como la de la figura 1.3. 


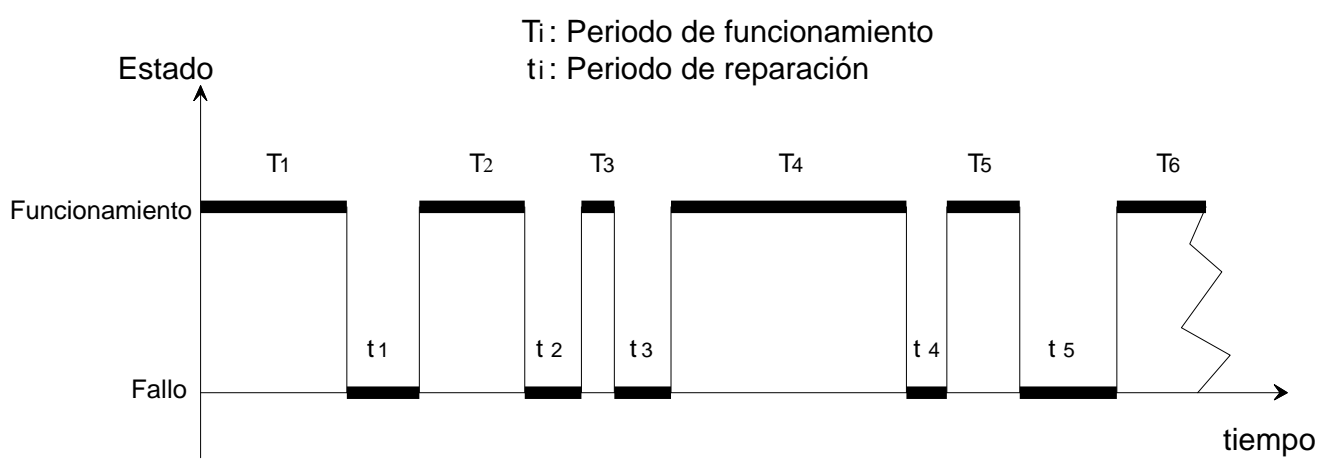

Fig.1.3.-Secuencias de operación-fallo de un componente.

Si denominamos $T_{i}$ a la duración de los intervalos de funcionamiento y $t_{i}$ a la de los intervalos de reparación, es inmediato deducir que la disponibilidad media del componente $A$, se puede expresar como (L_BIL-RIN-73) :

$$
A=\frac{\sum_{i} T_{i}}{\sum_{i}\left(T_{i}+t_{i}\right)}
$$

La indisponibilidad media $U$, como: $\quad U=\frac{\sum_{i} t_{i}}{\sum_{i}\left(T_{i}+t_{i}\right)}=1-A$

El tiempo total que el componente ha estado operativo será: $\quad \sum_{i} T_{i}$

El tiempo total de inoperatividad será: $\quad \sum_{i} t_{i}$

\subsubsection{Disponibilidad de Conjuntos de Componentes en Serie}

Las agrupaciones de componentes que constituyen un sistema serie constituyen un caso muy interesante desde nuestro punto de vista, pues las redes eléctricas que vamos a considerar se explotan de forma radial, pudiendo reducirlas a un sistema de elementos en serie.

Supongamos un sistema compuesto por $n$ elementos dispuestos en serie. Para que funcione el sistema habrán de funcionar todos y cada uno de los $n$ componentes. Así tendremos que para que el sistema se encuentre disponible en un instante dado, todos los elementos deben encontrarse disponibles. Y el tiempo acumulado de reparación del sistema serie, será la suma de los tiempos de reparación de cada componente, menos la duración de los intervalos en los que, al menos, dos elementos se encuentren, a un mismo tiempo, en reparación. 
Tomando como punto de partida las secuencias de dos componentes dispuestos en serie, lo anterior queda ilustrado en la figura 1.4.

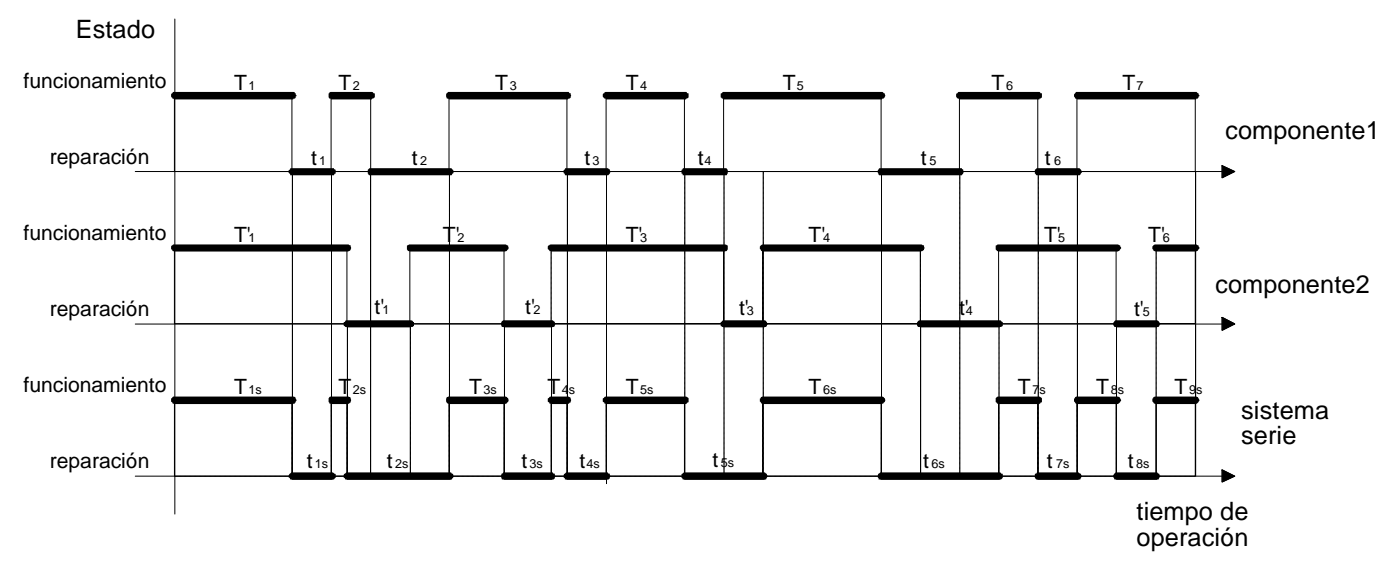

Fig.1.4.-Secuencia equivalente de dos componentes en serie

\subsection{4 Índices de Calidad de Servicio}

Para evaluar la calidad de servicio de una red de distribución, las compañías eléctricas utilizan una serie de parámetros denominados Índices de Calidad de Servicio. Éstos, básicamente, darán una idea acerca del tiempo de indisponibilidad, del número de interrupciones del servicio, o de la energía que se ha dejado de suministrar durante las mismas.

Existen dos tipos de interrupciones del suministro:

- Programadas. Son interrupciones previstas. Pueden deberse a mantenimiento preventivo (siempre que produzca indisponibilidad), a mantenimiento correctivo programado (por ejemplo, para volver a reparar completamente un elemento que ha sido puenteado previamente), o a interrupciones programadas de cualquier otro tipo (por ejemplo, el enganche de nuevos abonados a la red.)

- Imprevistas. Son debidas a averías en algún componente de la red. Como ya vimos, según su duración, podían ser transitorias o permanentes.

Cada uno de estos tipos de incidencias van a dar lugar a índices de calidad diferentes que convendrá analizar por separado, ya que a la hora de llevar a la práctica políticas de mejora de la calidad de servicio de la red, la actuación será totalmente diferente en uno u otro caso. Así en el caso de interrupciones programadas, los índices serán de carácter permanente. En el caso de la averías imprevistas, los índices considerados podrán ser temporales o permanentes. En esta Tesis, sólo se utilizarán los índices relacionados con las interrupciones de servicio imprevistas de carácter permanente. 
Por último, hay que destacar que existen dos formas de cálculo de los índices de calidad de servicio. Una primera, a posteriori, es decir, partiendo de los datos históricos de indisponibilidades en la red sobre un período de explotación ya concluido. Es la que realizan las compañías eléctricas. La segunda, a priori, que es la que se desarrollará en esta Tesis, toma como datos de partida las estimaciones de las tasas de fallo y de los tiempos de reposición asociados a cada componente. Esta metodología nos permitirá predecir los efectos producidos sobre los índices de calidad de servicio, fruto de las diversas actuaciones en los componentes y en la topología de la red.

\section{Índices de Calidad de Servicio de carácter Permanente}

Consideraremos representativos los índices: TIEPI, TIEB, NIEPI, NIEB, IIS, ENS, y K, que describiremos brevemente (M_CAL-96).

\section{TIEPI}

Tiempo de Interrupción Equivalente de la Potencia Instalada, en un período de tiempo determinado. La evaluación de este índice se realiza mediante la expresión:

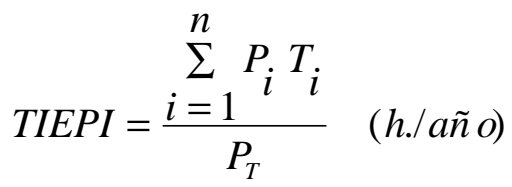

donde:

\begin{tabular}{|ll|}
\hline$n$ & número total de puntos carga de la instalación \\
\hline$P_{i}$ & potencia instalada en el iésimo punto de carga \\
\hline$T_{i}$ & tiempo acumulado de duración de las interrupciones en el punto de carga $i$ \\
\hline$P_{T}$ & potencia instalada total en la red \\
\hline
\end{tabular}

TIEB

Tiempo de Interrupción Equivalente de Cliente de Baja tensión, en un período de tiempo determinado. Se puede calcular a través de la siguiente expresión:

$$
T I E B=\frac{\sum_{i=1}^{n} N_{i} T_{i}}{N_{T}} \quad \text { (h./año }
$$

donde:

\begin{tabular}{|ll|}
\hline$n$ & número de puntos carga de la instalación \\
\hline$N_{i}$ & número de clientes que se abastecen del punto carga $i$ \\
\hline$T_{i}$ & tiempo acumulado de duración de las interrupciones en el punto de carga $i$ \\
\hline$N_{T}$ & número total de clientes que dependen de la instalación \\
\hline
\end{tabular}


NIEPI

Número de Interrupciones Equivalentes de la Potencia Instalada en un período de tiempo considerado. La formulación de este índice se expresa como:

$$
\text { NIEPI }=\frac{\sum_{i=1}^{n} P_{i} I_{i}}{P_{T}} \quad \text { (interrupciones / año) }
$$

donde:

\begin{tabular}{|ll|}
\hline$n$ & número de puntos carga de la instalación \\
\hline$I_{i}$ & número acumulado de interrupciones en el punto carga $i$ \\
\hline$P_{i}$ & potencia instalada el punto de carga $i$ \\
\hline$P_{T}$ & potencia total instalada en la red \\
\hline
\end{tabular}

NIEB

Número de Interrupciones Equivalentes de Clientes de Baja tensión en un período de tiempo considerado. La evaluación de este índice se realiza mediante la expresión:

$$
N I E B=\frac{\sum_{i=1}^{n} N_{i} I_{i}}{N_{T}} \quad \text { interrupciones / año) }
$$

donde:

\begin{tabular}{|ll|}
\hline$n$ & número de puntos carga de la instalación \\
\hline$I_{i}$ & número acumulado de interrupciones en el punto carga $i$ \\
\hline$N_{i}$ & número de clientes que se abastecen del punto de carga $i$ \\
\hline$N_{T}$ & número total de clientes que dependen de la instalación \\
\hline
\end{tabular}

IIS

Índice de Indisponibilidad de Suministro en un período de explotación considerado, expresado en unidades por mil. Se calcula mediante la expresión:

$$
I I S=\frac{T I E P I \cdot 1000}{H} \quad(\text { u.p.m. })
$$

donde:

\begin{tabular}{|ll|}
\hline$T I E P I$ & es el $T I E P I$ del período considerado $H$ \\
\hline$H$ & período de tiempo considerado \\
\hline
\end{tabular}


Energía No Suministrada en un período de tiempo determinado. Para evaluarla se utiliza la expresión:

$$
E N S=\frac{T I E P I \cdot E S}{H} \quad(M w \cdot h / a \tilde{n} o)
$$

donde:

\begin{tabular}{|ll|}
\hline TIEPI & es el TIEPI del período considerado $H$ \\
\hline$E S$ & energía suministrada en el período considerado $H$ \\
\hline$H$ & período de tiempo considerado \\
\hline
\end{tabular}

$K$

Número de incidencias de carácter permanente que se han producido en la instalación en un período de tiempo determinado. Se calcula mediante la expresión:

$$
K=\sum_{i=1}^{n} I_{i} \quad \text { (interrupciones / año) }
$$

donde:

\begin{tabular}{|ll|}
\hline$n$ & número de puntos carga de la instalación \\
\hline$I_{i}$ & número acumulado de interrupciones en el punto carga $i$ \\
\hline
\end{tabular}

Por su especial adecuación al tratamiento del tipo de problemas que nos ocupan, utilizaremos estos índices, con preferencia a otros típicos de la caracterización del comportamiento de sistemas genéricos tales como Tiempo Medio hasta el Fallo MTTF (Mean Time to Failure), Tiempo Medio entre Fallos MTBF, etc, de frecuente uso en la literatura (L_BIL-ALL-92).

\subsection{GRUPOS MÍNIMOS DE CORTE}

\subsubsection{Redes de Fiabilidad}

\section{Concepto de Redes de Fiabilidad}

La representación inmediata de un sistema físico de distribución de energía eléctrica es su red topológica, que abarca a todos los componentes que intervienen, así como su forma de conexión. Esta estructura esta sometida a una serie de lógicas de funcionamiento y actuación de protecciones que van a dar lugar a la red lógica correspondiente. Así, la red lógica nos indicaría la direccionalidad de los componentes, o la forma de actuación de las protecciones, junto con la secuencia de actuaciones para la reposición del servicio. 


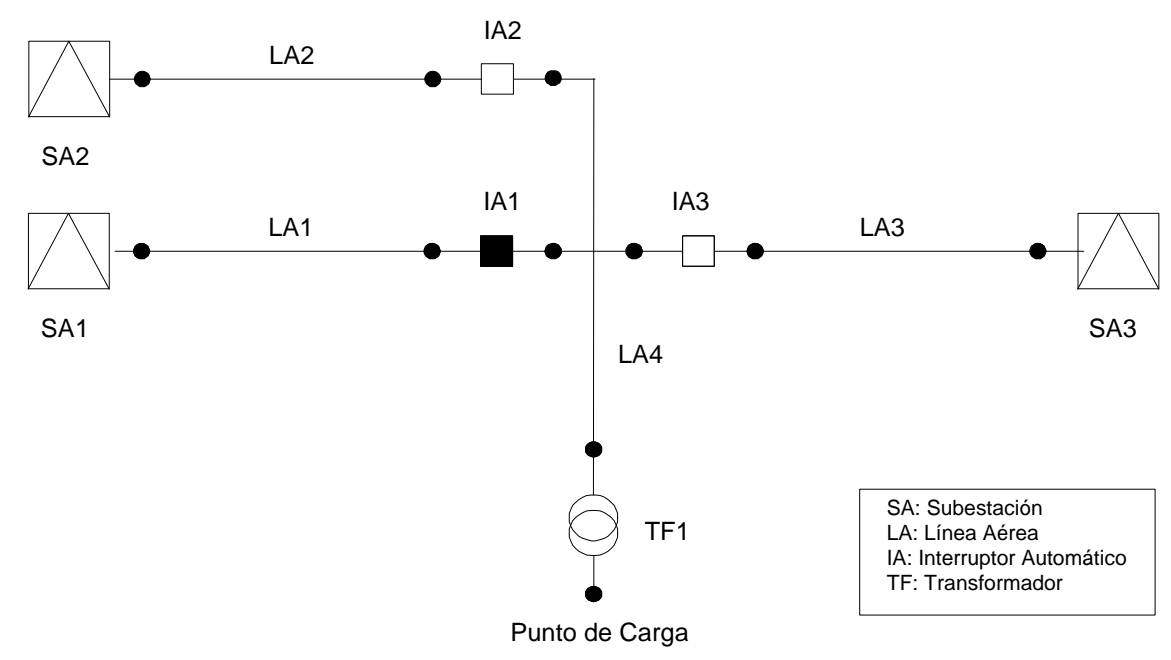

Fig.1.5.-Red topológica de un sistema

Dado un sistema representado por sus redes topológica y lógica, podemos definir la red de fiabilidad correspondiente, como el diagrama lógico que permite determinar el funcionamiento o fallo de dicho sistema, a partir de conocimiento del funcionamiento o fallo de un subconjunto del mismo.

Si en una red, constituida por sus estructuras lógica y topológica, tomamos un punto como entrada, al que llamaremos punto de análisis, diremos que el punto analizado 'funciona', cuando pueda establecerse al menos un camino hacia cualesquiera alimentación existente con capacidad suficiente para establecer un flujo de potencia. Dicho sistema 'fallará' cuando por avería de uno o más componentes sea imposible establecer el camino de circulación citado.

El concepto de red topológica puede entenderse fácilmente a partir de la figura 1.5, en la que describimos una red aérea con tres alimentaciones, de las que parten líneas aéreas protegidas por interruptores automáticos que confluyen a una línea que alimenta un transformador al que se conectarán los consumidores. Si la explotación de la red es radial, en el punto de carga solamente se recibe potencia de una subestación, estando las otras en situación de reserva por si falla la que se encuentre suministrando energía en un momento dado.

La red lógica correspondiente a esta red topológica vendría dada por la descripción del funcionamiento de toda la instalación: dirección del flujo de potencia, forma de actuación de los interruptores ante el fallo, actuaciones para la reposición de servicio, etc.

Si escogemos como punto de análisis el punto de carga, nos encontramos con una red de fiabilidad que representamos en la figura 1.6. 


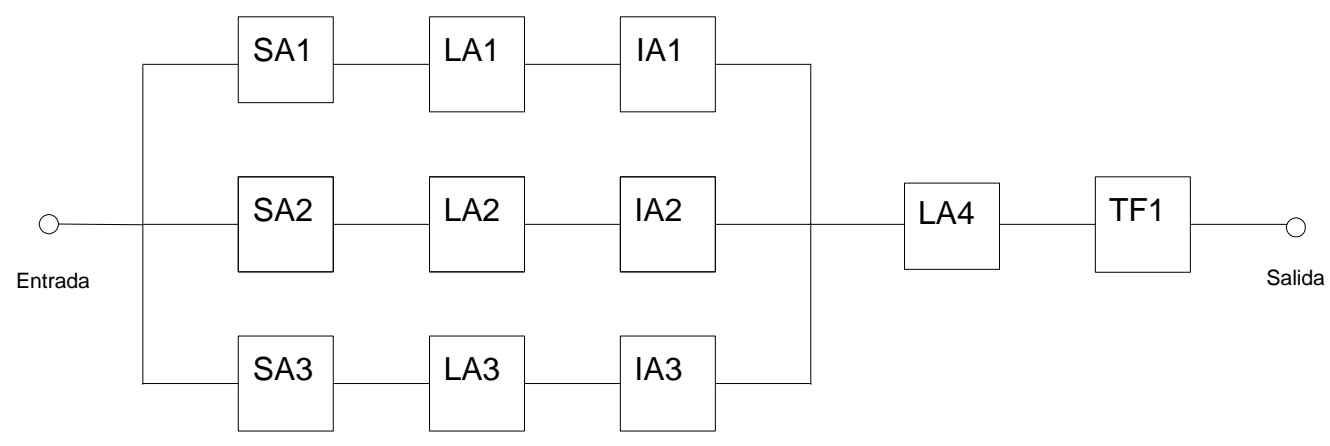

Fig.1.6.-Red de fiabilidad para el punto de carga señalado en la red de la figura 1.5.

En la figura 1.6 se puede ver que el punto de análisis que definimos en el punto de carga, puede resultar alimentado por tres caminos diferentes, que convergen en un camino común hacia la carga, en serie con la estructura anterior. Si falla, bien este último tramo, o bien los otros tres en paralelo a la vez, el punto analizado deja de encontrarse abastecido.

\section{Disposiciones Básicas de Elementos}

Podemos distinguir, desde el punto de vista de la fiabilidad, tres tipos básicos de disposición de elementos: serie, paralelo, y apoyo.

Consideraremos siempre que la explotación de las redes de distribución es radial, característica que dará lugar sistemáticamente a configuraciones serie, en las que el fallo de un elemento provoca el fallo del conjunto. Sin embargo, la estructura física de la red suele ser mallada, especialmente en redes urbanas, hecho que implica la existencia de configuraciones de apoyo, o partes de la red que, mediante maniobras, podrán suplir el fallo en uno de los componentes evitando el fallo del conjunto.

Finalmente, la disposición paralelo, que dividiremos en dos. Paralelo totalmente redundante, cuando basta que funcione un elemento para que funcione el conjunto (o dicho de otra manera, que han de fallar todos los elementos para que deja de funcionar el conjunto). Y Paralelo parcialmente redundante, cuando han de funcionar parte del número total de componentes para que el conjunto funcione.

La disposición en paralelo aparece muy raras veces, y en puntos muy localizados, como centros de transformación o subestaciones. En esta Tesis, estas instalaciones se encuentran descritas como un único elemento, por lo que no consideraremos la disposición en paralelo.

\subsubsection{Análisis de Redes. Método de los Grupos Mínimos de Corte}


Las primeras técnicas para calcular la fiabilidad en un determinado punto de una red eléctrica (A_GAV-MON-64) se basaban en la reducción sucesiva de elementos. Esta técnica agrupa los elementos por parejas en configuraciones serie y paralelo, para hallar la tasa de fallo equivalente de cada pareja, y volver a agruparlos repitiendo todo el proceso, hasta llegar a tener un sólo elemento equivalente.

A pesar de los buenos resultados que proporciona para redes eléctricas sencillas, se muestra insuficiente a la hora de analizar redes eléctricas complejas y es preciso acudir a técnicas más sistemáticas y potentes, y que sean, además, de fácil implementación en una computadora digital. Así, en la bibliografía (L_BIL-ALL83) encontramos como técnica básica (con diversos nombres pero con los mismos fundamentos), el Método de los Grupos Mínimos de Corte (a partir de ahora GMC). A lo largo del presente apartado describiremos muy brevemente esta metodología.

\section{Caminos Mínimos}

Un conjunto de componentes constituyen un Camino entre dos puntos de la red cuando el funcionamientos de todos ellos garantiza el funcionamientos de la red. Dicho camino tendrá la característica de Mínimo, cuando el fallo de uno cualesquiera de los componentes provoca el fallo de la red entre ambos puntos.

En términos de estructura lógica, el conjunto de todos los caminos mínimos constituye una estructura paralelo redundante; a su vez, cada camino esta compuesto por elementos dispuestos en serie.

\section{Grupos Mínimos de Corte}

Se denomina Grupo de Corte entre dos puntos de una red, a un conjunto de componentes cuyo fallo simultáneo provoca el fallo del sistema. Al igual que ocurría con los caminos, un grupo de corte es Mínimo cuando se cumple que, si uno de los componentes de dicho grupo de corte funciona, el sistema funciona.

Desde el punto de vista de la estructura lógica, los distintos caminos mínimos se disponen en una configuración paralelo redundante, mientras que los GMC, entre sí, forman una estructura serie, estando a su vez, cada GMC, compuesto por elementos dispuestos en configuración paralelo redundante

Se denomina Orden de un GMC al número de elementos que constituyen dicho grupo. Cada uno de los elementos constituyentes se denomina Subgrupo de Corte.

\section{Cálculo de Fiabilidad a partir de los GMC}


Desde el punto de vista lógico, los GMC se disponen en serie. Sin embargo a priori, es incorrecto calcular la fiabilidad del conjunto como si se tratase de un sistema serie, esto es, sumando la contribución de cada GMC a la fiabilidad total. En nuestro caso, cada componente puede aparecer más de una vez en la red de fiabilidad (formando parte de varios GMC), con lo que los GMC no son independientes entre si (L_BIL-ALL-92).

Tendremos que aplicar entonces, el concepto de unión de sucesos. Denominando al suceso "fallo de un GMC de orden i" (que estará provocado por el fallo de todos sus componentes) como $G_{i}$. Si razonamos en término de infiabilidad, el fallo del sistema estará provocado por la unión de dichos sucesos:

$$
Q=p\left(\bigcup_{i} G_{i}\right)
$$

Si consideramos GMC de orden mayor que dos, la expresión final, resultado de desarrollar la probabilidad de la unión de los GMC, llega a ser muy compleja. Debiéndose introducir algunas aproximaciones que reduzcan los cálculos, garantizando un error mínimo, especialmente, cuando los datos de fiabilidad para todos los componentes son del mismo orden.

La aproximación que adoptaremos es considerar sólo los GMC de orden uno, despreciando los de orden superior. Esta aproximación es perfectamente válida por varias razones (M_CAL-96):

- El número de GMC de orden superior a uno, será normalmente pequeño, especialmente en redes aéreas (de tipo rural) ya que están poco malladas.

- Los componentes poseen una elevada fiabilidad, por lo que si ya es poco probable el fallo de un componente, mucho menos lo será el fallo de un segundo que constituya un GMC de segundo orden junto con otros. Esto hace que la contribución de estos GMC a la indisponibilidad del sistema sea muy pequeña.

- Además, el error es cualitativamente mucho menor que el provocado por la incertidumbre de los datos de fiabilidad de partida.

Por tanto, al quedarnos sólo con los GMC de orden uno podremos calcular la fiabilidad del conjunto como si se tratase de un sistema serie.

\section{Aplicación a las Redes de Distribución}

Concretaremos la técnica de los GMC expuesta hasta ahora para el caso de Redes de Distribución de energía eléctrica.

Cuando el componente, en todo momento, sólo tiene asociada una única probabilidad de fallo, los GMC se corresponden con componentes, como ya hemos dicho. En nuestro caso, los componentes tienen asociadas varias probabilidades de fallo y por tanto los GMC no corresponden exactamente a componentes, sino que 
representan sucesos que ocurren asociados a esos componentes. En el caso de los GMC de orden uno, cada uno de dichos sucesos viene representado por:

- Un componente que falla, con dos tasas de fallo asociadas, una para cada estado atmosférico.

- Por el modo de reposición del servicio para el punto de carga analizado cuando se produce el fallo en el componente asociado al GMC. A este modo de reposición le asociamos un tiempo de reposición.

Debido a la actuación de las protecciones y a la lógica de funcionamiento, la obtención de los de los GMC de orden 1 no es tan evidente como hemos expuesto en el apartado anterior. Distinguiremos dos tipos de GMC de orden 1: topológicos y lógicos.

- Topológicos. Constituyen estos GMC de orden uno todos aquellos sucesos (fallo de un componente) que provocan indisponibilidad en el punto de carga.

- Lógicos. Provocan indisponibilidad en el punto de carga, fruto de la acción de las protecciones y de la lógica de funcionamiento de la red. Son aquellos sucesos de orden uno, cuyos componentes asociados están conectados al camino principal de alimentación, siempre que entre este y aquellos, no haya ningún elemento de protección.

\subsection{SIMULACIÓN. MÉTODO DE MONTE CARLO}

Como define Shannon (L_SHA-75), "Simulación es el proceso de diseñar y desarrollar un modelo computerizado de un sistema o proceso y conducir experimentos con este modelo con el propósito de entender el comportamiento del sistema o evaluar varias estrategias con las cuales se puede operar el sistema".

Normalmente, la simulación se contemplaba, tradicionalmente, como un "último recurso", que se empleaba cuando las técnicas analíticas no eran capaces de resolver los problemas. Sin embargo, los avances en la metodologías de la simulación, así como la disponibilidad del software y el desarrollo tecnológico de los ordenadores, han hecho de la simulación una de las herramientas más ampliamente usadas y aceptadas en el análisis de sistemas.

Habíamos definido la simulación como una técnica para llevar a cabo ensayos de experimentos en el modelo de un sistema (L_CEL-91). Esta definición general se lleva frecuentemente a la práctica mediante la técnica conocida como Simulación por el Método de Monte Carlo. El término Monte Carlo fue introducido por Von Neumann y Ulam, durante el transcurso de la Segunda Guerra Mundial (L_RUB-81), como una palabra clave para designar una nueva técnica de resolución de un modelo utilizando números aleatorios, que se aplicó a problemas relacionados con el desarrollo secreto de la Bomba Atómica en Los Álamos (EE.UU.). Este nombre fue sugerido por su analogía con los casinos de juego existentes en la ciudad de Montecarlo, en Mónaco (L_SOB-76). Y a partir de entonces, debido a que el ensayo 
a partir de una distribución particular lleva consigo el uso de números aleatorios, la simulación estocástica se denomina con frecuencia simulación de Monte Carlo. Estrictamente hablando, es incorrecto aplicar esta denominación a procesos que no sean completamente aleatorios en todos los aspectos (como el lanzamiento de una moneda). Así, deberíamos hablar de simulación estocástica para los procesos estocásticos, esto es, para procesos dependientes del o con el tiempo, y que por tanto no reúnen todas las características de aleatoriedad como para ser etiquetados con el término Monte Carlo.

Se puede citar muchas situaciones en las que la simulación puede ser utilizada con éxito (L_MAT-92) . Mencionaremos algunas de ellas.

- En primer lugar, puede ser imposible o extremadamente caro obtener datos de ciertos procesos en el mundo real. En este caso decimos que los datos simulados son necesarios para formular hipótesis acerca del sistema.

- En segundo lugar, el sistema observado puede ser tan complejo que resulte prácticamente imposible describirlo en términos de un sistema de ecuaciones matemáticas cuya solución analítica sea obtenible.

- En tercer lugar, aunque pueda formularse un modelo matemático que describa el sistema a analizar, puede no ser posible obtener solución al modelo mediante técnicas analíticas directas.

- Por último, puede ser imposible o muy costoso llevar a cabo la validación de experimentos según los modelos matemáticos que describen el sistema. En este caso diremos que los datos de simulación pueden ser utilizados para verificar hipótesis alternativas. 


\subsection{GENERACIÓN DE VARIABLES ALEATORIAS UNIFORMES.}

Dos aspectos fundamentales, y que no suelen conceptuarse como significativos en los experimentos de simulación estocástica, son, por un lado la influencia que tiene el generador de números aleatorios en la uniformidad y aleatoriedad del conjunto de números aleatorios generados y, por otro, la confiabilidad y el grado de exactitud que estas dos características transmiten a los resultados (L_COS-82).

En nuestro experimento de simulación necesitamos generar valores de variables aleatorias, que representen tiempos que se distribuyan según una cierta ley de probabilidad que, a su vez, representan el funcionamiento real de cada componente de la red. El objetivo será conseguir que cada una de estas secuencias se ajuste lo más posible a una distribución de probabilidad prefijada, y por tanto al modelo de simulación propuesto para dicha realidad.

El proceso de generar un valor de la variable aleatoria de una distribución particular, se repetirá tantas veces como se desee y tantas veces como distribuciones de probabilidad existan en el experimento de simulación. Sin embargo, el proceso por el que generamos variables aleatorias no uniformes parte de la generación de una distribución uniforme, para convertirla, más tarde, en la distribución que necesitemos mediante alguno de los métodos existentes. Entre los métodos más comunes y difundidos podemos mencionar el método de la transformada inversa, el método de rechazo o Neumann, el método de composición, y los procedimientos especiales (L_KNU-81) (L_RUB-81) (L_DAG-88). Por tanto la bondad en la generación las 
variables aleatorias uniformes repercutirá, posteriormente cuando sea transformada a una distribución particular, en una correcta obtención de los ciclos de funcionamiento de cada componente del sistema a simular.

Las distribuciones aleatorias uniformes son secuencias de números aleatorios que se distribuyen en un rango especificado (típicamente entre 0 y 1 ), de manera que cualquiera de los números comprendidos en este rango tiene la misma probabilidad de ser generado. La generación de esta distribución aleatoria uniforme debe poseer ciertas características deseables que aseguren o aumenten la confiabilidad de los resultados obtenidos en la simulación (L_COS-82) (L_SOB-76). Tales características son:

- Uniformemente distribuidos: esto es, que los números aleatorios generados estén exactamente equiespaciados y ninguno se repita. Esta característica es esencial.

- Estadísticamente independientes: esto es, que no exista ningún tipo de correlación estadística entre los números generados, ni individualmente ni en ninguna forma en que se les agrupe (en parejas, ternas, etc.). En otras palabras, dos cualesquiera secuencias diferentes de números aleatorios, deberían producir los mismos resultados cuando se utilizan en una aplicación particular. Esta característica es esencial.

- Reproducibles: Las secuencias de números aleatorios deben poder ser generadas exactamente de nuevo. Esta característica no es esencial, aunque sí muy deseable.

- Período largo: que los números no se repitan dentro de una longitud determinada de la sucesión. La longitud del período dependerá de la aplicación a la que se destinen lo aleatorios generados.

- Generados a través de un método que no requiera mucha capacidad de almacenamiento en la computadora. Esta característica es deseable pero no esencial a la generación.

Finalmente, señalemos que algunos autores, por ejemplo (L_KNU-81), califican a los números aleatorios generados a través de relaciones de recurrencia como números pseudoaleatorios, por ser una sucesión de dígitos generada mediante una regla puramente determinista y que jamás cumplen escrupulosamente las características de uniformidad e independencia que les son consustanciales. Sin embargo, desde un punto de vista pragmático podemos considerar que una sucesión de números es aleatoria si satisface en cierto grado, que variará según la aplicación para la que se genere la sucesión, las características de uniformidad e independencia que un cierto conjunto de pruebas estadísticas se encargará de "probar". Esto es, no importa que sean esencialmente aleatorios, sino que accidentalmente se comporten como tales.

\section{Generadores de variables aleatorias uniformes}


A continuación y dada la gran importancia que en nuestro experimento de simulación tiene una correcta generación de secuencias de números aleatorios uniformes, estudiaremos detenidamente la conveniencia de utilizar diversas rutinas, implementadas en computadora, para generar dichas secuencias, aunque, como ya dijimos, resulta paradójico el hecho de utilizar una computadora, la más precisa y determinista de las máquinas para producir números aleatorios; después de todo, cualquier programa produce resultados totalmente predecibles. Para realizar este estudio utilizaremos distintas pruebas sobre un conjunto de ocho diferentes rutinas para elegir la más convenientes a nuestros propósitos.

La mayoría de las versiones del lenguaje de programación ' $\mathrm{C}$ ' tienen rutinas para, inicializar una semilla, y a partir de ahí, generar una secuencia de números aleatorios. Cada semilla dará lugar a una secuencia diferente de números entero comprendidos en un rango entre 0 y un valor prefijado, normalmente 32767. Este tipo de rutinas generalmente se denominan $\operatorname{rand}($ ).

En principio, y dada la experiencia histórica reseñada en (L_PRE-TEU-92), nos acercaremos con cierta desconfianza hacia los generadores de números aleatorios del propio sistema. Éstos, son normalmente del tipo lineal congruencial, es decir, generan la secuencia de números enteros entre 0 y m-1 mediante la relación de recurrencia (A_LEH-51):

$$
I i+1=a I i+c-m k i
$$

donde:

$" m$ " se denomina módulo

" $a$ " y " $c$ " son constantes enteras positivas, llamadas multiplicador e incremento respectivamente.

$k i=(a I i+c) / m$ denota el mayor entero positivo contenido en el cociente $(a I i+c) / m$.

La recurrencia se repetirá con un valor, obviamente no superior a $\mathrm{m}$. Si las constantes " $a$ ", " $c$ ", y " $m$ " son elegidas de forma apropiada, entonces el periodo tendrá la máxima longitud posible, es decir m . En este caso, todos los enteros posibles entre 0 y m-1 ocurren en algún momento. Así cualquier semilla inicial, es tan buena como otra.

Los métodos lineales congruentes tienen la ventaja de ser muy rápidos al requerir pocas operaciones por cada llamada; esto los hace ser aceptados universalmente. Sin embargo hay algunos problemas típicos que se podrían citar (L_PRE-TEU-92):

- Algunas aplicaciones pueden necesitar una secuencia mucho mayor que la obtenida mediante esta rutina. Esta dificultad lleva a plantearse la implementación de otras rutinas que tengan un período mucho mas grande. Es evidente que si se quiere disponer de $10^{6}$ números aleatorios diferentes para alguna aplicación específica, no será lo mismo utilizar una función que genere 
números todos distintos entre sí, que otra que suministre los mismos 32.767 números 30 veces.

- Un segundo obstáculo, éste ya propio de las implementaciones particulares, es que la elección de las constantes del modelo puede no ser particularmente acertada.

- Otra desventaja que tienen estos generadores es que no están exentos de correlación secuencial entre las sucesivas llamadas. Si k números aleatorios generados se usan para representar un punto en el espacio k-dimensional (con cada coordenada comprendida entre 0 y 1 ), entonces los diversos puntos generados no tenderán a rellenar el espacio de manera uniforme, sino que más bien se dispondrán en planos $(k-1)$-dimensionales, habiendo como mucho $\mathrm{m}^{(1 / \mathrm{k})}$ planos de este tipo. Además, si las constantes " $a$ ", " $c$ ", y " $m$ " no están bien elegidas tendremos, todavía, muchos menos planos. Así, podríamos estar focalizando la atención de un proceso en una pequeña fracción del volumen total.

Cuando en el algoritmo lineal congruencial se elige la constante de incremento " $c$ " como cero, obtenemos el algoritmo lineal congruencial simplemente multiplicativo. Se tiene constancia de que este algoritmo puede ser tan bueno como cualquiera de los que tienen " $c$ " distinto de cero, siempre que " $a$ "y " $m$ " sean elegidos con esmero.

La traducción de este algoritmo al lenguaje C, está basado en la implementación desarrollada por Schrage (A_SCH-79) y se basa en la elección de constantes de Park y Miller (A_PAR-MIL-88), se denominará ranO( ). El período de ranO( ) es de $2^{31}-2$, aproximadamente igual a $2,1 * 10^{9}$. Esta rutina es un mínimo estándar para la mayoría de las aplicaciones.

Existe otra correlación secuencial entre números sucesivos, más sutil, presente en $\operatorname{ranO(~).~Si~se~representan~puntos~sucesivos~en~un~plano~bidimensional,~entonces~la~}$ distribución resultante no supera el test de chi-cuadrado (la llamada prueba de las series cuadradas) cuando $\mathrm{N}$ es algo mayor que $10^{7}$, cantidad mucho menor que el período m-2. La siguiente rutina $\operatorname{ranl}()$ usa el método mínimo estándar, pero añadiendo un proceso de mezclado de la salida para intentar eliminar la correlación citada. El j-ésimo valor de la secuencia sale, no en la j-ésima llamada a la rutina sino en una llamada posterior (aleatoriamente), que en promedio será j+32. Este algoritmo de "mezcla", es debido a Bays y Durham (A_BAY-DUR-76) y está estudiado por Knuth (L_KNU-81).

En la rutina $\operatorname{ran2(})$ implementamos una rutina desarrollada por L`Ecuyer (A_LEC-88) que combina dos secuencias diferentes con períodos distintos, con el fin de obtener una nueva secuencia cuyo período sea el mínimo común múltiplo de ambos. Con una elección adecuada de las constantes, el período del generador combinado puede llegar a ser de $2,3 * 10^{18}$ aproximadamente. Aunque la mezcla de 
dos secuencias deshace en gran medida las correlaciones en serie; no obstante empleamos el proceso de mezcla adicional ya empleado en $\operatorname{ranl}($ ).

Otra rutina del mismo tipo es la denominada $\operatorname{ran6}($ ), desarrollada inicialmente por Marse y Roberts (A_MAR-ROB-83). La principal ventaja de ésta es la utilización de las operaciones de desplazamiento de bits, en lugar de las operaciones aritméticas usuales (suma y resta), con el consiguiente aumento en la velocidad de generación.

En la rutina ran3( ) implementamos una sugerencia debida a Knuth (L_KNU81) acerca de una rutina portable, y que posee una mecánica totalmente diferente a las anteriores.

También implementamos un sistema de generación de números aleatorios basado en la criptografía de datos (L_PRE-TEU-92), que dio lugar a una rutina no estrictamente portable, que denominaremos $\operatorname{ran4}($ ), ya que asume que los enteros largos sin signo tienen 32 bits, lo que sucede en la mayoría de las maquinas, pero no en todas. Esta rutina actúa sobre 64 bits de entrada, aplicando de manera iterativa una función de mezclado de bits que es altamente no lineal. Esta función se denomina "de cifrado" y consiste en un intrincado conjunto de permutaciones actuando sobre pequeños grupos de bits consecutivos.

Se ha probado también otra función de generación de números aleatorios que aparece implementada en el ANSI-C de la estación de trabajo empleada en los experimentos (HP-720). Se denomina drand48( ) y tiene un período de $2^{48}$. Es un algoritmo de tipo lineal congruencial pero con una aritmética de enteros de 48 bits (a diferencia de los anteriores que utilizaban enteros de 32 bits). Proporciona un valor entre 0 y 1 (no incluyendo este último). Tiene el inconveniente de no ser portable a otro tipo de máquinas.

\subsection{PRUEBAS SOBRE LOS GENERADORES DE VARIABLES ALEATORIAS UNIFORMES.}

Dada la importancia que tiene en nuestro estudio conseguir un buen generador de números aleatorios, que nos garantice la aproximación a la solución exacta en el menor tiempo de computo posible, someteremos a todos los generadores de números aleatorios descritos anteriormente a un conjunto de pruebas estadísticas y prácticas para comprobar si cumplen las características de aleatoriedad, uniformidad y periodicidad que debería verificar un generador ideal. Estas pruebas las realizamos repetidamente en un ordenador personal Intel-486 y en una estación de trabajo Hewlet Packard 720. Para programar tanto los generadores de aleatorios como las pruebas a que fueron sometidos, se utilizó el lenguaje de programación ANSI-C.

\section{Pruebas estadísticas.}


Las pruebas estadísticas se dividen en dos (L_COS-82): las que comprueban si los números generados se distribuyen uniformemente y las que comprueban su independencia estadística. Ambos tipos de pruebas estadísticas se pueden aplicar globalmente (generando un tamaño de muestra igual al período del generador) $o$, como hicimos, localmente (generando tamaños de muestra menores al período), pues en la simulación empleamos tamaños de muestra menores al período. Estas pruebas (L_ARN-78) se basan en la teoría de contraste de hipótesis simple y utilizan como hipótesis algunas de las características del cálculo de probabilidades que cumplen los números aleatorios. siguiente:

El método de actuación para el contraste de una hipótesis simple es el

1. Se obtiene una muestra aleatoria de tamaño n.

2. Se calcula la discrepancia existente entre el valor mantenido por la hipótesis y el que resulta de la muestra.

3. Suponiendo que la hipótesis es cierta, se calcula la probabilidad de obtener una discrepancia mayor o igual que la encontrada.

4. Si la probabilidad de obtener dicha desviación es menor que un cierto valor fijado de antemano -nivel de significación-, se rechaza la hipótesis.

Se ve entonces que el fundamento de este tipo de contrastes es el criterio de que las cosas poco probables no deben suceder.

Esta pruebas son: la prueba de los promedios, la de frecuencias, la de distancias, la de series cuadradas y cúbicas, y la de acotado superior e inferior. Los fundamentos estadísticos de estas pruebas los comentamos brevemente a continuación.

\section{- $\quad$ Prueba del promedio}

Esta prueba se basa en que el valor medio o esperado de una variable aleatoria uniformemente distribuida es igual a 0.5 (L_COS-82). Por tanto deberemos probar que "no es rechazable la hipótesis de que los números pseudoaleatorios que generamos provienen de un universo uniforme con media de 0.5 ".

La prueba se realiza generando una muestra de tamaño $\mathrm{N}$ y evaluando el promedio aritmético de los números obtenidos, $\hat{x}$. A continuación se construye el estadístico:

$$
\mathrm{Z}=\sqrt{12 \mathrm{~N}}(\hat{\mathrm{x}}-0.5)
$$

que, si la hipótesis nula $\hat{x}=0.5$ es cierta, se distribuye según una t de Student de N-1 grados de libertad. 
Después de fijar un nivel de significación " $\alpha$ ", se determinará si el valor de Z es menor que un cierto valor $Z_{o}$ que cumple, para la distribución t de Student, que $\mathrm{P}(|\mathrm{t}| \geq$ $\mathrm{Z}_{\mathrm{o}}$ ) = " $\alpha$ ". Si es así, no se puede rechazar la hipótesis de que los números que generamos tienen una media de 0.5 .

\section{- $\quad$ Prueba de frecuencias}

Esta es una de las pruebas más importantes para el contraste de hipótesis sobre una distribución cualquiera. En líneas generales (L_KNU-81), opera dividiendo la distribución observada en $\mathrm{n}$ intervalos para luego comparar el número total de sucesos observados en cada intervalo con el número teórico que resultaría de provenir ésta de la distribución teórica con la que se compara.

En nuestro caso, dividiremos el intervalo $(0,1)$ en n subintervalos iguales, para comparar luego el número de pseudoaleatorios que se observa dentro de cada subintervalo $\mathrm{FO}_{\mathrm{i}}$ con el número de los que teóricamente debería haber $\mathrm{FE}_{\mathrm{i}}$-esto será el cociente del número total de pseudoaleatorios generados por el número de subintervalos-. Cuando ambas cantidades son muy parecidas, la muestra proviene de un universo uniforme.

Este procedimiento lo ilustramos en la figura 2.1, donde hemos subdividido el intervalo $(0,1)$ en 10 subintervalos iguales, y donde podemos apreciar la manera en la que se ha distribuido una secuencia de aleatorios.

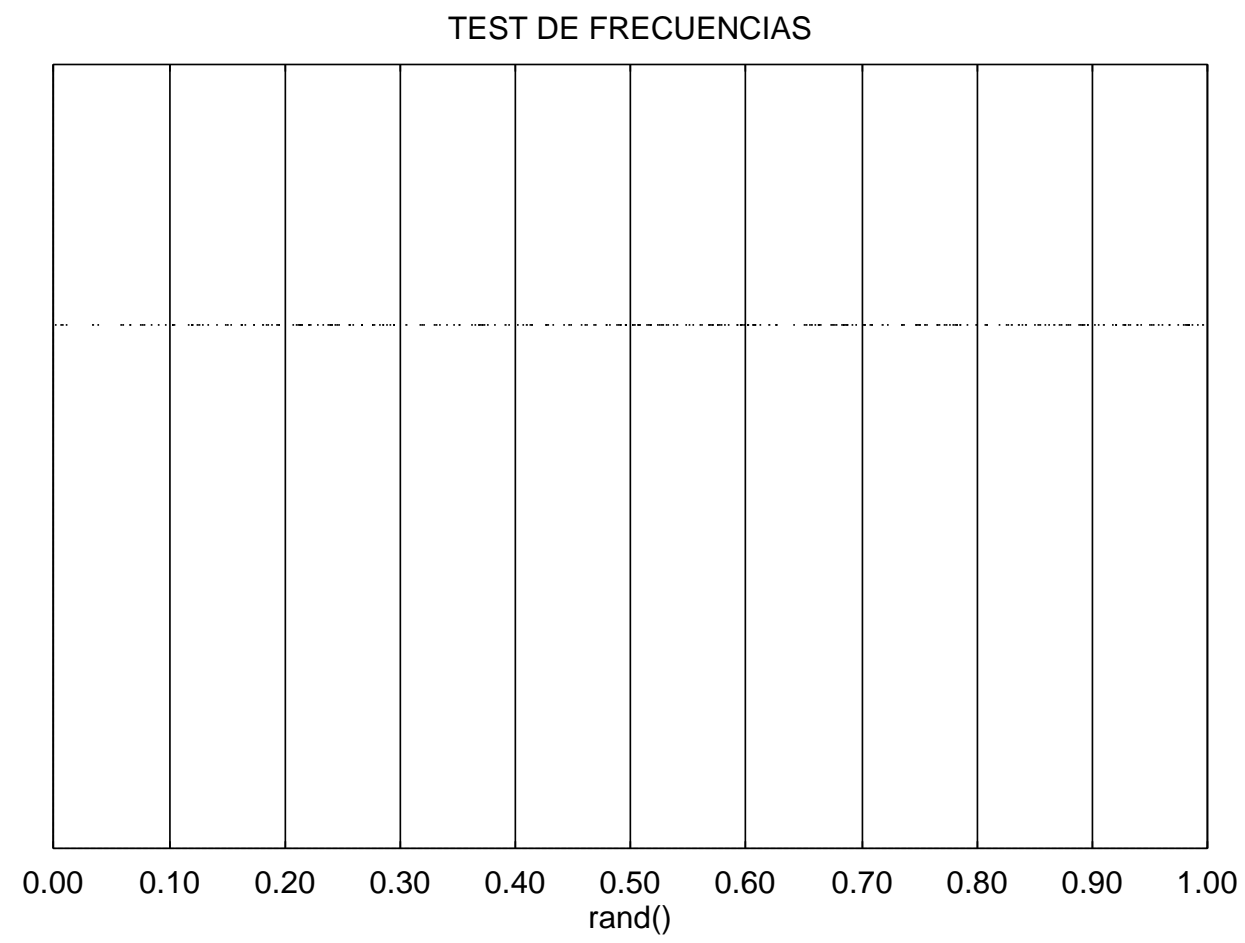

Fig.2.1.-Test de frecuencias para un generador de números aleatorios 
El estadístico que se utiliza en esta prueba es:

$$
X=\sum_{i=1}^{n} \frac{\left(F O_{i}-F E_{i}\right)^{2}}{F_{i}}
$$

que, si la hipótesis nula es cierta, se distribuye según una chi-cuadrada con (n-1) grados de libertad.

Después de fijar un nivel de significación " $\alpha$ ", se determinará si el valor de $X$ es menor que un cierto valor $X_{o}$ que cumple, para la distribución chi-cuadrada, que $\mathrm{P}\left(|\mathrm{t}| \geq \mathrm{X}_{\mathrm{o}}\right)=\alpha$. Si es así, no se puede rechazar la hipótesis de uniformidad. Para la correcta aplicación de esta prueba debemos asegurarnos de que el número de subintervalos elegido no sea tan grande que provoque que el número esperado de ocurrencias en un subintervalo sea menor que cinco.

\section{- $\quad$ Prueba de distancias}

Para realizar las modalidad de esta prueba en que se considera al número pseudoaleatorio como un número real (L_KNU-81) es preciso seleccionar un intervalo $(\mathrm{A}, \mathrm{B})$ que se halle contenido en $(0,1)$. Para cada número aleatorio generado se comprueba si pertenece o no al intervalo $(A, B)$. Si el j-ésimo número pertenece al intervalo $(A, B)$, y hasta el $(j+i+1)$-ésimo, que también pertenece al intervalo $(A, B)$, no ha habido otro que pertenezca a este intervalo, se dice que tenemos un hueco de tamaño i. La distribución de probabilidad del tamaño de estos huecos, siendo $\mathrm{N}$ el número total de números pseudoaleatorios generados y por tanto el producto $\mathrm{z}^{*} \mathrm{~N}$, donde $\mathrm{z}=\mathrm{A}-\mathrm{B}$, el número esperado de ellos en el intervalo $(\mathrm{A}, \mathrm{B})$, es:

$$
\begin{aligned}
& F E_{i}=z(1-z)^{i}(z N) \\
& F E_{i} \geq_{n}=(1-z)^{n}(z N) \quad i=0,1,2, \ldots
\end{aligned}
$$

Es importante señalar que el valor seleccionado de $n$, que es la mayor anchura de hueco permitida, debe ser tal que la suma de las apariciones esperadas de todos los tamaños de huecos agrupados $\mathrm{FE}_{\mathrm{i} \geq \mathrm{n}}$ sea mayor que 5. También, reseñar que el resultado de esta prueba no depende del intervalo $(\mathrm{A}, \mathrm{B})$ elegido.

Con las cantidades esperadas y observadas se construye el mismo estadístico que en la prueba anterior que, si la hipótesis nula fuera cierta, se distribuiría según una chi-cuadrada con $\mathrm{n}$ grados de libertad.

- $\quad$ Prueba de series cuadradas y series cúbicas

Estas dos pruebas (L_KNU-81) se utilizan para comprobar el grado de aleatoriedad entre números sucesivos; la de series cuadradas entre dos números consecutivos y la de series cúbicas entre tres. 
En la prueba de series cuadradas se forman parejas de números consecutivos, las cuales se consideran coordenadas en la superficie de un cuadrado unitario dividido en $\mathrm{n}^{2}$ celdas. Se contabiliza la cantidad de pseudoaleatorios que se encuentran en cada celda $\mathrm{FO}_{\mathrm{ij}}$ y se compara con la cantidad esperada $\mathrm{FE}_{\mathrm{ij}}$ dada por $(\mathrm{N}-1) / \mathrm{n}^{2}$, donde $\mathrm{N}$ es el número de pseudoaleatorios generados. Luego, se construye el estadístico:

$$
X_{o}=\sum_{i=1}^{n} \sum_{j=1}^{n} \frac{\left(F O_{i j}-F E_{i j}\right)^{2}}{F E_{i j}}
$$

que, si la hipótesis nula fuera cierta, se distribuiría según una chi-cuadrada con $\mathrm{n}^{2}-1$ grados de libertad. En la figura 2.2 podemos apreciar la manera en la que se ha distribuido una secuencia de aleatorios, donde las parejas de números consecutivos se consideran coordenadas en la superficie de un plano unitario $(0,1) \times(0,1)$ que hemos subdividido en 100 subplanos iguales.

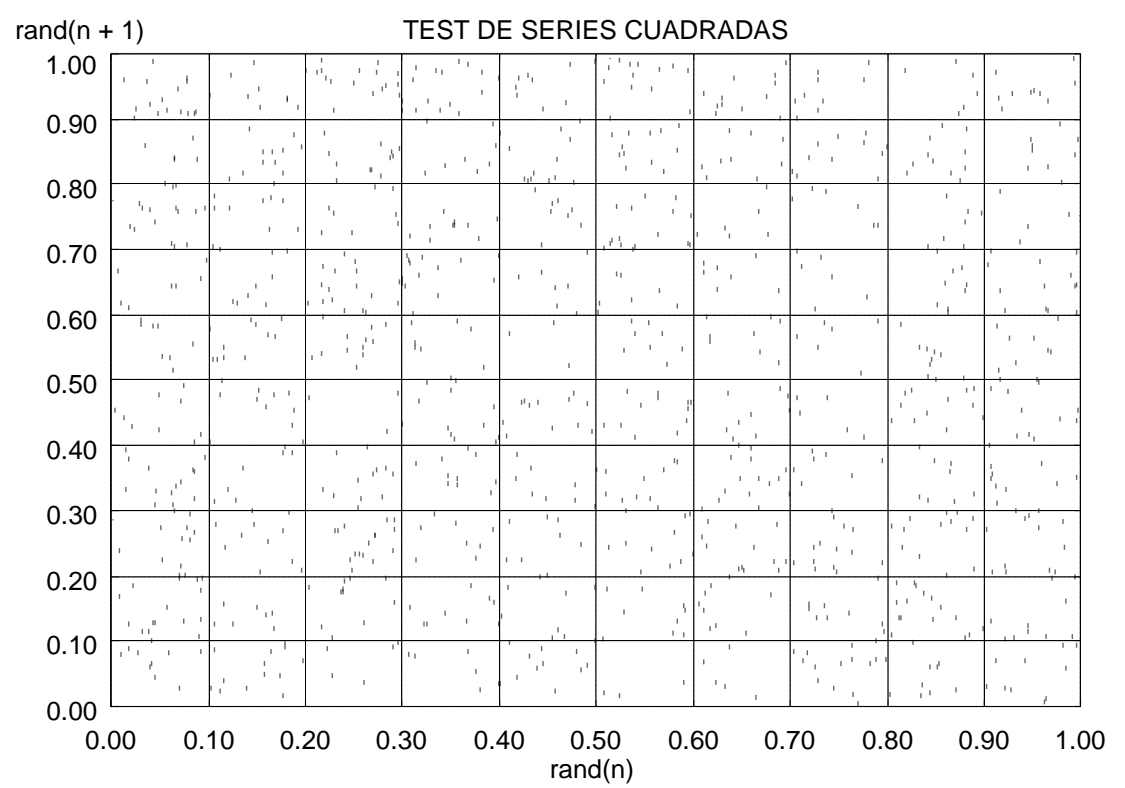

Fig.2.2.-Test de series cuadradas para un generador de números aleatorios

En la prueba de series cúbicas se forman ternas de números consecutivos, los cuales se consideran coordenadas espaciales en el volumen de un cubo unitario dividido en $\mathrm{n}^{3}$ celdas. Nuevamente se contabiliza la cantidad de pseudoaleatorios que se encuentran en cada celda $\mathrm{FO}_{\mathrm{ijk}}$ y se compara con la cantidad esperada $\mathrm{FE}_{\mathrm{ijk}}$ dada por $(\mathrm{N}-2) / \mathrm{n}^{3}$, donde $\mathrm{N}$ es el número de pseudoaleatorios generados. Luego, se construye el estadístico:

$$
X_{o}=\sum_{i=1}^{n} \sum_{j=1}^{n} \sum_{k=1}^{n} \frac{\left(F O_{i j k}-F E_{i j k}\right)^{2}}{F E_{i j k}}
$$

que, si la hipótesis nula fuera cierta, se distribuiría según una chi-cuadrada con $\mathrm{n}^{3}-1$ grados de libertad. 
En la figura 2.3 podemos apreciar la forma en la que se ha distribuido una secuencia de aleatorios, donde las ternas de números consecutivos se consideran coordenadas en la superficie de un cubo unitario $(0,1) \times(0,1) \times(0,1)$ que hemos subdividido en 125 subcubos iguales.

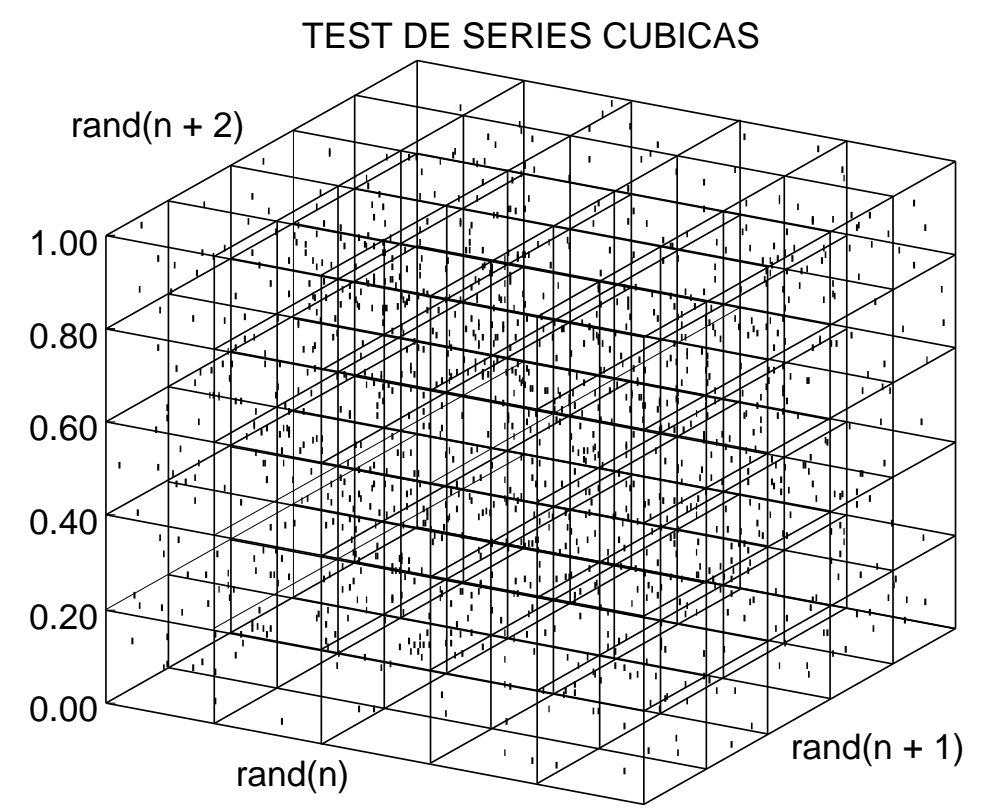

Fig.2.3.-Test de series cúbicas para un generador de números aleatorios

- $\quad$ Prueba de acotado superior e inferior

En esta prueba (L_NAY-66), a partir de la secuencia de $\mathrm{N}$ números pseudoaleatorios generados $x_{i} i=1,2, \ldots, N$, se construye una secuencia de ceros $y$ unos en la cual el i-ésimo término es 0 si $\mathrm{x}_{\mathrm{i}}<\mathrm{x}_{\mathrm{i}+1} \mathrm{y} 1$ en caso contrario. Una vez obtenida esta secuencia binaria se determina el número de carreras de longitud $\mathrm{j}$, entendiendo por tales la sucesión consecutiva y sin interrupción de j ceros o j unos, indistintamente. Se contabiliza entonces la cantidad de carreras de cada longitud y se compara con la cantidad teórica, que viene dada por:

$$
\begin{array}{ll}
F E_{i}=2 \frac{N\left(i^{2}+3 i+1\right)-\left(i^{3}+3 i^{2}-i-4\right)}{(i+3) !} & i<N-1 \\
F E_{N-1}=2 / N ! & i=N-1
\end{array}
$$

Nuevamente, con las cantidades esperadas y observadas se construye el estadístico 1.2.2., que, si la hipótesis nula fuera cierta, se distribuiría según una chicuadrada con n-1 grados de libertad, donde $n$ es la mayor longitud de corrida permitida. 


\subsection{GENERACIÓN DE VARIABLES ALEATORIAS NO-UNIFORMES.}

En los apartado anteriores de este capítulo nos centramos especialmente en el proceso de generación de variables aleatorias con una distribución de probabilidad uniforme. Este interés es debido a que el proceso por el cual generamos variables aleatorias no uniformes parte de la generación de una distribución uniforme, para convertirla, más tarde, en la distribución que necesitemos mediante alguno de los métodos más comunes y difundidos, entre los que podemos mencionar el método de la transformada inversa, el método de rechazo o Neumann, el método de composición, y procedimientos especiales (L_KNU-81) (L_RUB-81) (L_DAG-88). A continuación explicaremos el método de la transformada inversa, ya que es el más utilizado y el que elegimos para obtener los dos tipos de distribuciones que vamos a necesitar, la exponencial y la normal.

El método de la transformada inversa utiliza la función distribución de probabilidad $F(x)$ de la variable aleatoria que se va a simular. La función de distribución $F(x)$ nos da la probabilidad de que un número cualquiera $X$, obtenido al azar, sea mayor que $x$ :

$$
F(x)=\text { probabilidad de que }(X \leq x)=p(X \leq x)
$$

La función de distribución crece monótonamente desde cero a uno; o sea

$$
F\left(x_{1}\right) \leq F\left(x_{2}\right) \quad \text { si } \quad x_{1} \leq x_{2} ; \quad F(-\infty)=0, \quad F(+\infty)=1
$$

Si $F(x)$ es continua y estrictamente creciente, toma valores entre cero y uno, y existe una función inversa $F^{-1}(y)$ tal que:

$$
\forall 0<y<1, \quad y=F(x) \Leftrightarrow x=F^{-1}(y)
$$

Entonces se puede generar un número aleatorio uniforme $U$ y tratar de determinar el valor de la variable aleatoria para la cual su distribución acumulada es igual a $U$. Es decir, la variable aleatoria $X=F^{-1}(U)$ tiene por función de distribución a $F(x)$.

La prueba es sencilla:

$$
p(X \leq x)=p\left[F^{-1}(U) \leq x\right]=p[U \leq F(x)]=F(x)
$$

El principal inconveniente de este método reside en la dificultad de encontrar analíticamente la transformada inversa de la función de distribución $F(x)$ de la variable aleatoria que queremos obtener. En estos casos se puede recurrir al calculo numérico, aunque ralentice el proceso de simulación. 
Aplicaremos este método para conseguir generar números aleatorios distribuidos según una distribución exponencial y una distribución normal.

\section{Distribución exponencial}

Queremos generar números al azar que sigan la distribución exponencial:

$$
f(x)= \begin{cases}\lambda e^{-\lambda x} & \text { si } x \geq 0 \\ 0 & \text { si } x<0\end{cases}
$$

La distribución acumulada de esta distribución es:

$$
F(x)=\int_{0}^{x} \lambda e^{-\lambda x} d t=1-e^{-\lambda x}
$$

e igualando la distribución acumulada con el número uniforme $U$, se obtiene:

$$
1-e^{-\lambda x}=U \Rightarrow e^{-\lambda x}=1-U \Rightarrow x=-\frac{1}{\lambda} \ln (1-U)
$$

Pero si $U$ sigue una distribución uniforme $1-U$ también la seguirá. Por consiguiente:

$$
x=-\frac{1}{\lambda} \ln U
$$

\section{Distribución normal}

El método polar debido a Box, Muller y Marsaglia (A_BOX-MUL-58) (A_MAR-62) para generar una distribución normal dice que las variables aleatorias independientes $X_{1}$ y $X_{2}$ se distribuyen según una normal.

$$
X_{1}=V_{1} \sqrt{\frac{-2 \ln S}{S}}, \quad X_{2}=V_{2} \sqrt{\frac{-2 \ln S}{S}}
$$

donde:

$$
\begin{aligned}
& S=V_{1}^{2}+V_{2}^{2} \quad \text { siempre que } S<1 \\
& V_{1}=2 U_{1}-1 \\
& V_{2}=2 U_{2}-1 \\
& U_{1} y U_{2} \text { son variables aleatorias uniformes }
\end{aligned}
$$

Para probar la validez de este método, utilizaremos conceptos elementales de geometría y calculo: $\mathrm{Si} S<1$, el punto en el plano cartesiano definido por las coordenadas $\left(V_{1}, V_{2}\right)$ es un punto uniformemente distribuido dentro de un círculo de radio unidad. Transformando las coordenadas de este punto a coordenadas polares 
tenemos que $V_{1}=R \cos \Theta \quad$ y $\quad V_{2}=R \cos \Theta$. Sustituyendo en las ecuaciones anteriores tendremos que:

$$
\begin{aligned}
& S=R^{2} \Rightarrow R=\sqrt{S} \\
& X_{1}=\sqrt{S} \cos \Theta \sqrt{\frac{-2 \ln S}{S}}=\sqrt{-2 \ln S} \cos \Theta \\
& X_{2}=\sqrt{S} \operatorname{sen} \Theta \sqrt{\frac{-2 \ln S}{S}}=\sqrt{-2 \ln S} \operatorname{sen} \Theta
\end{aligned}
$$

Utilizando, también, las coordenadas polares para representar a $X_{1}$ y $X_{2}$ podemos escribir que $X_{1}=R^{\prime} \cos \Theta^{\prime} \quad$ y $\quad X_{2}=R^{\prime} \cos \Theta^{\prime}$. Si igualamos con las ecuaciones anteriores tenemos que:

$$
\begin{aligned}
\Theta^{\prime} & =\Theta \\
R^{\prime} & =\sqrt{-2 \ln S}
\end{aligned}
$$

Se ve claramente que $R^{\prime}$ y $\Theta^{\prime}$ son independientes, ya que $R$ y $\Theta$ lo eran dentro del circulo de radio unidad. La probabilidad de que $R^{\prime} \leq r$ es igual a $1-e^{-r^{2} / 2}$ porque $S$ $=R^{2}$ está uniformemente distribuido entre cero y uno:

$$
\mathrm{P}\left(\mathrm{R}^{\prime} \leq \mathrm{r}\right)=\mathrm{P}\left(-2 \ln \mathrm{S} \leq \mathrm{r}^{2}\right)=\mathrm{P}\left(\mathrm{S} \geq \mathrm{e}^{-\mathrm{r}^{2} / 2}\right)=1-\mathrm{e}^{-\mathrm{r}^{2} / 2}
$$

La probabilidad de que $R^{\prime}$ se halle entre $r$ y $r+d r$ es la derivada de $1-e^{-r^{2} / 2}$ o sea $r e^{-r^{2} / 2} d r$

También $\Theta^{\prime}$ está uniformemente distribuida entre 0 y $2 \pi$, por tanto la probabilidad de que $\Theta$ ' se halle entre $\theta$ y $\theta+d \theta$ es igual a $(1 / 2 \pi) d \theta$. La probabilidad conjunta de que $X_{1} \leq x_{1}$ y de que $X_{2} \leq x_{2}$ queda como:

$$
\begin{aligned}
\int_{\left\{(r, \theta) \mid r \cos \theta \leq x_{1}, r \operatorname{sen} \theta \leq x_{2}\right\}} \frac{1}{2 \pi} e^{-r^{2} / 2} r d r d \theta & = \\
& =\frac{1}{2 \pi} \int_{\left\{(x, y) \mid x \leq x_{1}, y \leq x_{2}\right\}} e^{-\left(x^{2}+y^{2}\right) / 2} d x d y \\
& =\left(\sqrt{\frac{1}{2 \pi}} \int_{-\infty}^{x_{1}} e^{-x^{2} / 2} d x\right)\left(\sqrt{\frac{1}{2 \pi}} \int_{-\infty}^{x_{2}} e^{-y^{2} / 2} d y\right)
\end{aligned}
$$

Esto prueba que $X_{1}$ y $X_{2}$, como los hemos definido, son independientes y se distribuyen normalmente, como deseamos.

\subsection{DISEÑO DEL EXPERIMENTO DE SIMULACIÓN.}


Una vez contemplado el estudio estadístico de los generadores de números aleatorios y elegido el generador que hemos denominado ran6( ), el siguiente paso será el diseño de nuestro experimento de simulación y, como en cualquier diseño de experimentos, el objetivo será optimizar la exactitud de los resultados y el tiempo de cálculo que empleará el programa de simulación en obtener esos resultados. Para optimizar estos dos parámetros conjuntamente deberemos llegar a un compromiso entre la simplificación y la escrupulosa representación de las características de funcionamiento del sistema real que queremos modelizar.

Para ello probaremos con dos modelos distintos de menor a mayor complejidad. En ambos modelos, calcularemos la disponibilidad de un modelo muy simple de sistema eléctrico mediante el método de simulación estocástica secuencial, y compararemos el resultado obtenido con la solución analítica estacionaria. En una primera aproximación no consideraremos que las condiciones meteorológicas influyen en el sistema, que incluiremos en una segunda aproximación; sin perder nunca de vista que la pretendida exactitud obtenida por un número elevado de simulaciones en cualquiera de las dos aproximaciones puede resultar vana cuando tenemos valores estimados de tasas de fallo y de reparación que quizás estén alejados de la realidad.

\subsubsection{Experimento sin considerar la influencia de las condiciones meteorológicas}

El sistema propuesto está tomado de (L_BIL-ALL-92), y consiste en dos componentes en paralelo, actuando en redundancia simple; es decir, basta con que funcione uno para que funcione el conjunto.

Los elementos son reparables, y se considera que, tanto su tiempo hasta el fallo, como su tiempo de reparación siguen una distribución exponencial negativa, siendo conocidas sus tasas medias de fallo y de reparación: $\lambda_{1}, \lambda_{2}, \mu_{1}, \mu_{2}$.de cada elemento. Estas tasas son constantes, no influyendo la variación de las condiciones meteorológicas en ellas. Los valores elegidos en este ejemplo son:

$$
\begin{array}{ll}
\lambda_{1}=0.0012 \mathrm{f} / \mathrm{h} & \lambda_{2}=0.002 \mathrm{f} / \mathrm{h} \\
\mu_{1}=0.003 \mathrm{r} / \mathrm{h} & \mu_{2}=0.0045 \mathrm{r} / \mathrm{h}
\end{array}
$$

La disponibilidad del sistema en su valor estacionario, esto es cuando $t \rightarrow \infty$, obtenida analíticamente aplicando procesos de Markov de espacio de estado discreto y parámetro continuo, viene dada por la ecuación:

$$
A(t \rightarrow \infty)=1-\frac{\lambda_{1} \lambda_{2}}{\left(\lambda_{1}+\mu_{1}\right)\left(\lambda_{2}+\mu_{2}\right)}
$$

que, para los valores considerados, dará un resultado de $A(t \rightarrow \infty)=0.912088$.

Veamos ahora lo que obtenemos por simulación. El tipo de simulación estocástica que empleamos es secuencial con incrementos de tiempo no constantes (según los eventos), de tal forma que se generan los intervalos de funcionamiento y 
reparación del sistema en orden cronológico hasta alcanzar un límite que llamaremos período de simulación.

El método consiste en generar dos secuencias de números aleatorios para cada uno de los componentes; una de ellas corresponderá a los intervalos de funcionamiento y la otra a los de reparación. Estas secuencias de tiempos, expresados en horas, las distribuiremos exponencialmente y se obtendrán mediante el método de la transformada inversa, expuesto en el apartado anterior.

Lo que obtendremos será un ciclo de operación-reparación para todo el sistema paralelo que podrá representarse como indica la figura 2.4., a partir de los ciclos de los elementos que componen ese sistema.

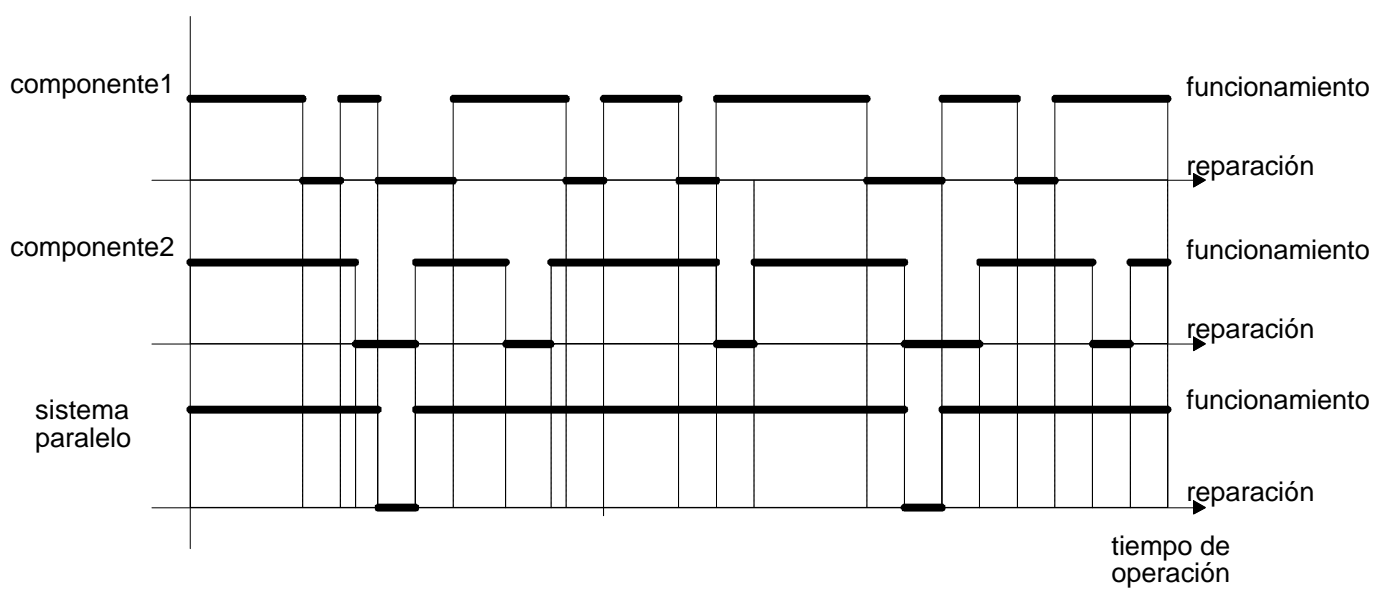

Fig.2.4.-Ciclos de operación-reparación de los dos componentes y el total del sistema.

El algoritmo del proceso de simulación por el que obtendremos la secuencia de ciclos funcionamiento-reparación del sistema paralelo a partir de los ciclos de sus dos elementos es el siguiente:

1. Generar un número aleatorio.

2. Convertir este número en un valor de tiempo de funcionamiento.

3. Generar otro número aleatorio.

4. Convertir este número en un valor de tiempo de reparación.

5. Repetir los pasos del $\mathbf{1}$ al $\mathbf{4}$ para el período de vida de simulación.

6. Repetir los pasos del $\mathbf{1}$ al $\mathbf{5}$ para cada componente.

7. Comparar las secuencias para cada componente y deducir los ciclos de funcionamiento-reparación para el sistema total, número total de interrupciones, disponibilidad acumulada, tiempo medio por interrupción, etc.

8. Repetir los pasos del 1 al 7 hasta alcanzar el período de simulación deseado.

A priori, consideramos dos formas de proceder para hallar la disponibilidad del sistema: 
- Fijar un período de simulación muy elevado, y observar la evolución temporal de la disponibilidad acumulada (es decir, hablando en términos de indisponibilidad, sería el tiempo de reparación acumulado, dividido entre el tiempo total transcurrido hasta ese momento). Este valor tendería a estabilizarse alrededor de una cantidad próxima al valor analítico estacionario.

- Fijar un período de simulación mucho menor y como contrapartida repetir el proceso un número prefijado de veces. Las disponibilidades tenderán a agruparse, distribuyéndose como una normal, alrededor del valor estacionario.

El desarrollo del experimento y sus resultados con estas dos formas de proceder, se recoge en el apartado 4.1.

\subsubsection{Experimento considerando la influencia de las condiciones meteorológicas}

La fiabilidad de las redes eléctricas se ve influenciada, significativamente, por las condiciones meteorológicas bajo las que trabajan, por lo que deberemos incluir este factor en nuestro modelo definitivo de simulación. Antes, trataremos de ensayar en un sistema simple el modelo que emplearemos, más tarde, para dar cuenta de la influencia de las condiciones meteorológicas en el comportamiento de una Red de Distribución y examinar su influencia sobre los índices de calidad de ésta.

Introduciremos el factor meteorológico en el modelo de simulación a través de aquellos componentes cuyo funcionamiento real pueda verse influido por el mismo; esta consideración afecta a todos los elementos de intemperie. Por tanto, en el análisis de fiabilidad tendremos en cuenta el efecto de las condiciones meteorológicas de la región geográfica en la que se encuentre situada la red, introduciendo una corrección en los parámetros de fiabilidad de los componentes.

Con este fin, se ha considerado que las condiciones meteorológicas determinarán dos posibles estados para los elementos de la Red que se encuentren a la intemperie (A_GAV-MON-64): Tiempo Normal y Tiempo Tormentoso (también denominados buen tiempo y mal tiempo, respectivamente), entendiendo como Tiempo Tormentoso aquel que causa una tasa de fallo anormalmente alta en los componentes expuestos al mismo, mientras que como Tiempo Normal entenderemos el resto de situaciones. También se ha considerado que todos los componentes de la Red de Distribución se encuentran sometidos a las mismas condiciones meteorológicas simultáneamente.

La determinación de los intervalos de tiempo en los que los elementos que se encuentran a la intemperie se hallan sometidos a buen y mal tiempo puede efectuarse de dos formas:

- Considerando los períodos reales de buen y mal tiempo obtenidos directamente de los datos históricos de la estación meteorológica de la zona en la que se encuentre la Red de Distribución. En el caso de que dichos 
históricos no abarcasen el total del tiempo de simulación requerido, se repetirían las mismas secuencias de manera cíclica.

- Analizando los datos meteorológicos para la obtención de la distribución estadística de las duraciones de los periodos de buen y mal tiempo, y sus correspondientes parámetros. Con éstos podríamos simular una serie de intervalos alternativos de buen y mal tiempo que representarían la evolución real de las condiciones meteorológicas a lo largo del tiempo de simulación como se muestra en la figura 2.5.

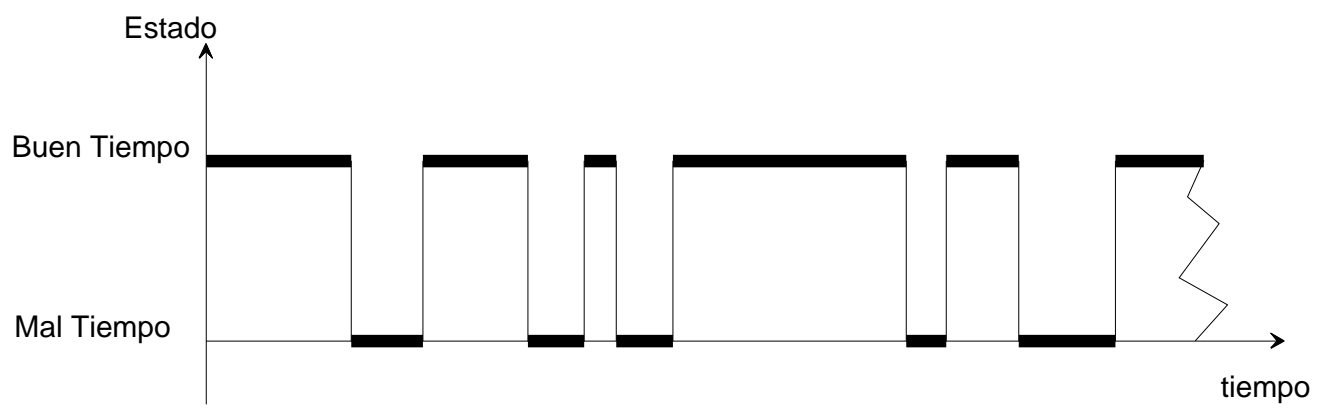

Fig.2.5.-Evolución en el tiempo de las condiciones meteorológicas.

En esta Tesis se ha utilizado la segunda de las alternativas anteriores; admitiendo que los periodos de buen y mal tiempo siguen una distribución exponencial (A_GAV-MON-64), con una duración media anual esperada de $N$ y $S$ horas respectivamente, $N$ y $S$ serán variables en la simulación. Al objeto de ver su influencia en los resultados, variaremos los parámetros del número medio de horas de buen y mal tiempo tanto en términos absolutos, como en cuanto a la relación entre ambos.

El sistema que hemos elegido, como en el experimento anterior en el que no considerábamos la influencia de las condiciones meteorológicas, consta de dos elementos genéricos, $A$ y $B$, colocados en paralelo, en redundancia total (basta que uno funcione para que el sistema funcione) con una lógica de funcionamiento correspondiente a componentes de intemperie, con faltas de tipo permanente. En la Tabla 2.1 se muestran los datos de fiabilidad de los componentes A y B según la forma en que dividamos el intervalo de tiempo que dura la reposición de servicio y la forma en que elijamos cómo se distribuyen las duraciones esperadas de los eventos que se producen en la Red.

\begin{tabular}{|c|c|c|}
\hline & Componente A & Componente B \\
\hline $\begin{array}{l}\text { Tasa de fallo para buen tiempo (fallos/hora) } \\
\text { (si el tiempo hasta el fallo se distribuye exponencialmente) }\end{array}$ & 0.0012 & 0.002 \\
\hline
\end{tabular}




\begin{tabular}{|c|c|c|}
\hline $\begin{array}{c}\text { Tasa de fallo para mal tiempo (fallos/hora) } \\
\text { (si el tiempo hasta el fallo se distribuye exponencialmente) }\end{array}$ & 0.12 & 0.2 \\
\hline $\begin{array}{l}\text { Tasa de reparación para mal tiempo ( reparaciones/hora) } \\
\text { (si el tiempo de localización y reparación se distribuyen exponencialmente) }\end{array}$ & 0.0 & 0.0 \\
\hline $\begin{array}{c}\text { Tasa de localización para mal tiempo (localizaciones/hora) } \\
\text { (si el tiempo de localización y reparación se distribuyen exponencialmente) }\end{array}$ & 0.0 & 0.0 \\
\hline $\begin{array}{c}\text { Media de la reparación para mal tiempo (horas) } \\
\text { (si el tiempo de localización y reparación se distribuyen normalmente) }\end{array}$ & 0.0 & 0.0 \\
\hline $\begin{array}{c}\text { Media de la localización para mal tiempo (horas) } \\
\text { (si el tiempo de localización y reparación se distribuyen normalmente) }\end{array}$ & 0.0 & 0.0 \\
\hline $\begin{array}{l}\text { Desviación de la reparación para mal tiempo (horas) } \\
\text { (si el tiempo de localización y reparación se distribuyen normalmente) }\end{array}$ & 0.0 & 0.0 \\
\hline $\begin{array}{c}\text { Desviación de la localización para mal tiempo (horas) } \\
\text { (si el tiempo de localización y reparación se distribuyen normalmente) }\end{array}$ & 0.0 & 0.0 \\
\hline $\begin{array}{l}\text { Tasa de reparación para buen tiempo ( reparaciones/hora) } \\
\text { (si el tiempo de localización y reparación se distribuye exponencialmente y } \\
\text { considerando la duración de la reposición como sólo de reparación) }\end{array}$ & 0.003 & 0.0045 \\
\hline $\begin{array}{l}\text { Tasa de localización para buen tiempo ( localizaciones/hora) } \\
\text { (si el tiempo de localización y reparación se distribuye exponencialmente y } \\
\text { considerando la duración de la reposición como localización + reparación) }\end{array}$ & 0.02 & 0.025 \\
\hline $\begin{array}{l}\text { Tasa de reparación para buen tiempo ( reparaciones/hora) } \\
\text { (si el tiempo de localización y reparación se distribuye exponencialmente y } \\
\text { considerando la duración de la reposición como localización + reparación) }\end{array}$ & 0.00353 & 0.00549 \\
\hline $\begin{array}{c}\text { Media de la reparación para buen tiempo (horas) } \\
\text { (si el tiempo de localización y reparación se distribuye normalmente y } \\
\text { considerando la duración de la reposición como sólo de reparación) }\end{array}$ & 333.33 & 222.22 \\
\hline $\begin{array}{l}\text { Desviación de la reparación para buen tiempo (horas) } \\
\text { (si el tiempo de localización y reparación se distribuye normalmente y } \\
\text { considerando la duración de la reposición como sólo de reparación) }\end{array}$ & 80 & 60 \\
\hline $\begin{array}{l}\text { Media de la localización para buen tiempo (horas) } \\
\text { (si el tiempo de localización y reparación se distribuye normalmente y } \\
\text { considerando la duración de la reposición como localización + reparación) }\end{array}$ & 50 & 40 \\
\hline $\begin{array}{l}\text { Desviación de la localización para buen tiempo (horas) } \\
\text { (si el tiempo de localización y reparación se distribuye normalmente y } \\
\text { considerando la duración de la reposición como localización + reparación) }\end{array}$ & 20 & 20 \\
\hline $\begin{array}{c}\text { Media de la reparación para buen tiempo (horas) } \\
\text { (si el tiempo de localización y reparación se distribuye normalmente y } \\
\text { considerando la duración de la reposición como localización + reparación) }\end{array}$ & 283.33 & 182.22 \\
\hline $\begin{array}{l}\text { Desviación de la reparación para buen tiempo (horas) } \\
\text { (si el tiempo de localización y reparación se distribuye normalmente y } \\
\text { considerando la duración de la reposición como localización + reparación) }\end{array}$ & 70 & 50 \\
\hline
\end{tabular}

Tabla 2.1.-Datos de fiabilidad de los componentes A y B.

Simularemos los ciclos de operación-fallo de dos maneras diferentes, que darán lugar a dos tipos de experimentos de simulación. En cualquiera de estas dos alternativas admitiremos que no se lleva a cabo reparación durante el mal tiempo y consideraremos además que el tiempo hasta el fallo (o tiempo de funcionamiento) seguirá una distribución exponencial con tasa de fallo conocida.

a) Como primera alternativa, consideraremos el intervalo de tiempo que dura la reposición de servicio de cada elemento, compuesto, sólo, por la duración de la reparación. Este intervalo de tiempo vendrá caracterizado por una tasa de reparación determinada. Simularemos dos casos: uno cuando el tiempo de reparación sigue una distribución exponencial, y otro cuando responde a una distribución normal, de media y desviación conocidas. Hay que indicar que, si 
se está efectuando una reparación (sólo posible durante el buen tiempo) y, súbitamente, cambian las condiciones meteorológicas, pasando a mal tiempo, habrá que abandonar la reparación y esperar a que llegue otra vez el buen tiempo para comenzarla, otra vez, desde el principio.

b) Una segunda alternativa, consistirá en dividir intervalo de tiempo que dura la reposición de servicio de cada elemento en dos subintervalos: el primero ocupa la localización de la avería más el desplazamiento hasta el lugar donde sucedió, y el segundo, consiste en la reparación de la misma. También simularemos dos casos: uno cuando el tiempo de reparación de los elementos sigue una distribución exponencial; y el otro caso, cuando responde a una distribución normal de media y desviación conocidas. Como en la anterior alternativa, consideraremos que si se está efectuando una reparación y, súbitamente, cambian las condiciones meteorológicas, pasando a mal tiempo, y ha sido posible finalizar la localización de la avería; cuando acabe el período de mal tiempo, no será necesario volver a llevar a cabo dicho proceso, realizando directamente al desplazamiento y reparación de la avería.

El desarrollo del experimento y sus resultados con estas dos formas de proceder, se recoge en el apartado 4.1.

\subsection{SIMULACIÓN DE EVENTOS EN LA RED}

Una red de distribución se ve afectada por las incidencias que se originan en sus componentes produciendo indisponibilidad en el propio componente y en los puntos de carga de la red, es decir, produciendo interrupción del servicio a los clientes que se alimentan de la misma a través de estos puntos de carga.

\section{Caracterización de los eventos de la red de fiabilidad}

A continuación se enumeran los factores que caracterizan a un evento de primer orden, dado por el fallo de un único componente bajo unas condiciones meteorológicas dadas y la reposición del servicio en un punto de carga. Los eventos se irán sucediendo mientras transcurre el tiempo, y los intervalos entre eventos vendrán caracterizados por sus distribuciones de probabilidad que, también, se describen para cada caso.

- Un componente que falla, con características propias.

- Un estado meteorológico, buen o mal tiempo, en el que el componente falla. Este estado meteorológico está caracterizado por las distribuciones de probabilidad del tiempo transcurrido hasta la aparición del mal tiempo, y tiempo transcurrido hasta la aparición del buen tiempo. En los modelos 
clásicos considera que ambas variables siguen una distribución exponencial. En esta Tesis se adoptará esta aproximación como la más adecuada.

- Un modo de fallo que lleva asociadas dos tasas de fallo, una por cada estado meteorológico que caracterizan la variable aleatoria tiempo hasta el fallo. La distribución de probabilidad del tiempo hasta el fallo de los componentes vendrá dada por una distribución exponencial negativa. Las hipótesis que se hacen tradicionalmente eliminan previamente los componentes defectuosos antes de su instalación en la Red y suponen que, una vez instalados, se les realiza un mantenimiento preventivo, suficiente como para que el componente se mantenga siempre dentro de su vida útil, con el objeto de mantener su tasa de fallo constante y la distribución de la variable aleatoria 'tiempo hasta el fallo' como una exponencial negativa. En esta Tesis mantendremos estas hipótesis.

- Modo de reposición del servicio para el punto de carga analizado cuando se produce el fallo, del tipo especificado, en el componente. Este modo de reposición lleva asociado un tiempo hasta la reposición del servicio. En los planteamientos clásicos de fiabilidad, en particular en las técnicas basadas en procesos homogéneos de Markov (A_GAV-MON-64) (A_BIL-BOL-68), se considera como una variable aleatoria de distribución exponencial negativa, para no romper el modelo de tasas de transición constantes entre estados, ya que esta aproximación facilita mucho la resolución del problema. Sin embargo, en el modelo de simulación que hemos utilizado en esta Tesis también vamos a considerar la distribución normal. Como ya se destacó en este capítulo, al proponer el modelo de simulación, el planteamiento seguido en esta Tesis acerca de la reposición del servicio consta de dos etapas: localización y desplazamiento hasta el punto donde tuvo lugar la avería; y reparación del componente (o cualquier otro modo de restauración). Consideraremos que la primera etapa se distribuye como una exponencial negativa (A_RIE-FRE-96); mientras que, podremos considerar que la duración de la segunda etapa se distribuye de la misma manera que la etapa anterior, o bien, podremos considerar la posibilidad de que se mueva en un rango de valores alrededor de una duración media propia de cada tipo de restauración para un componente determinado, esto es, podremos admitir que se distribuye normalmente, con una mayor o menor desviación, según sea el caso.

- Lógica de funcionamiento según el tipo de componente, el tipo de fallo, y el tipo de reposición que lleva consigo el suceso. Más adelante, en este apartado describiremos más en profundidad dichas lógicas.

\section{Modos de fallo y reparación.}

En el análisis de fiabilidad resulta necesario delimitar tanto el tipo de incidencias que pueden afectar a los componentes como el conjunto de acciones que permiten reponer el servicio (modos de reposición) en los puntos de carga. 
Cada componente de la red tiene dos estados posibles: funcionamiento y fallo, con independencia del factor meteorológico. A su vez, la duración de la reposición está compuesto de dos períodos diferenciados: localización de la avería con desplazamiento hasta el punto donde se encuentra, y reparación (o maniobra, si es el caso) de la misma. Caracterizaremos la indisponibilidad ocurrida en un punto de carga de la red, en un instante de tiempo determinado, por un modo de fallo del componente-s que provocan esa indisponibilidad, y por un tipo de reposición de esa indisponibilidad (M_CAL-96). En la Tabla 2.2 se presenta de forma esquemática el conjunto de eventos que afectan a la fiabilidad de una red de distribución tanto en lo que se refiere a las incidencias que provocan indisponibilidades como a las acciones de reposición que permiten superar esas indisponibilidades.

\begin{tabular}{|c|l|l|}
\hline \multicolumn{2}{|c|}{ Incidencias } & \multicolumn{1}{c|}{ Modos de Reposición } \\
\hline \multicolumn{1}{|c|}{ Tipo } & \multicolumn{1}{|c|}{ Causa } & Reenganche \\
\hline Programadas & Mantenimiento preventivo sin tensión & Fin de mantenimiento \\
\hline Imprevistas & Fallo temporal & $\begin{array}{l}\text { - Reparación/sustitución o } \\
\text { pseudosustitución. } \\
\text { - Aislamiento: } \\
\text { a) por protección. } \\
\text { b) por maniobra. } \\
\text { - Conmutación o transferencia de } \\
\text { carga. }\end{array}$ \\
\hline
\end{tabular}

Tabla 2.2.-Incidencias en Componentes y Acciones de Reposición del Servicio para los Puntos de Carga de la Red.

A primera vista puede parecer contradictorio el hecho de que cada modo de fallo tenga más de un modo de reposición. Sin embargo, lo que se simula son eventos de componentes para cada punto de carga. Así, si el fallo permanente de un componente afecta a dos puntos de carga diferentes, la reposición del servicio, en uno de ellos, puede resolverse mediante maniobra (transferencia de cargas), mientras que en el otro es posible que no quede más remedio que llevar a cabo la reparación del mismo. Será, por tanto, necesario analizar la forma en que cada fallo permanente afecta a cada punto de carga, y deducir las acciones de reposición que permiten restablecer el servicio en cada uno de los puntos de carga.

En esta Tesis sólo consideraremos el modo de fallo permanente, que es el más importante. Los fallos permanentes se originan por averías en componentes que provocan interrupciones de larga duración. El componente que sufre estos fallos quedará indisponible hasta que se realice la reparación o sustitución del mismo. Sin embargo, la indisponibilidad que este tipo de fallos produce en la red, no depende sólo del propio componente que la ha causado, sino que es función de la estructura de 
la misma y de que pueden existir soluciones alternativas de reposición del servicio, distintas a la de reparación del componente, como veremos posteriormente.

Los fallos permanentes se pueden producir en todos los componentes considerados (ver Anexo 1), excepto en los que tienen tasa de fallo nula (ver Anexo 2). Vienen cuantificados por una tasa media de fallo permanente. En las Líneas Aéreas, las tasas de fallo permanente en períodos de buen tiempo, son menores, como es lógico, que en los de mal tiempo. En los restantes elementos de intemperie, se han considerado iguales ya que se suponen perfectamente protegidos. En los componentes de interior, también coinciden la tasa de fallo en buen tiempo con la tasa de fallo en mal tiempo.

Con carácter general, cualquier fallo permanente de un componente en una red de distribución con esquema de explotación radial es susceptible de dividir a los puntos de carga de la misma, en relación con las diferentes acciones o modos de reposición, en tres grupos diferentes:

- Reposición por reparación o sustitución: es la restitución del servicio mediante la sustitución o la reparación completa de un componente. Durante los periodos de mal tiempo, sólo es posible llevarla a cabo en los elementos de interior, que posean este modo de reposición. Queda dividido en tiempo de localización y desplazamiento, y tiempo de reparación propiamente dicho, ambos representados por tasas medias que se expresan en horas.

- Reposición por pseudosustitución: consiste en la reposición del suministro mediante una reparación o sustitución del componente, que se efectúa con carácter provisional. La verdadera reparación con la total restitución de sus funciones, se realizará durante el mantenimiento programado. En el modelo propuesto de simulación, no contemplaremos el aspecto de la pérdida de funciones de protección o maniobra del elemento durante el tiempo en que permanece en ese estado. Al igual que en el modo de reposición por reparación, no es posible realizarlo durante el mal tiempo en los elementos de intemperie. Consta también de dos etapas: localización y desplazamiento, y reparación con las correspondientes tasas medias, expresadas en horas.

- Reposición por maniobra o accionamiento: asociado a acciones de conmutación, ya sea manual o automática, incluidas las maniobras de aislamiento de zonas de carga. Aparece en todos los elementos de maniobra y aislamiento (al menos en buen tiempo). Como ya hemos visto, se divide en dos etapas: localización y desplazamiento, y accionamiento propiamente dicho. En los elementos telemandados, el desplazamiento se considera cero.

\section{Lógica de funcionamiento de los componentes implicados en un evento}

A cada punto carga de la Red, le asociamos una red de fiabilidad compuesta por un conjunto de eventos dispuestos en serie, los Grupos Mínimos de Corte (GMC) de orden uno, en la forma descrita en el apartado 1.1. A modo de ejemplo, en la figura 2.6, se pueden ver los Caminos Mínimos asociados a los GMC de primer orden para el punto de carga número "0" de la red aérea. A continuación debemos 
generar las secuencias de funcionamiento-fallo de cada componente de los Caminos Mínimos para obtener la secuencia equivalente como describimos en el apartado 1.0.3. Esta secuencia total representa el comportamiento simulado de operación-fallo del punto de carga analizado. A partir del análisis de estas secuencias equivalentes por cada punto carga se obtiene la distribución estadística del tiempo hasta la interrupción y la de la duración de éstas; también obtendremos la indisponibilidad y el número de interrupciones de servicio para cada punto carga. Estos datos forman la información indispensable que, junto con las características funcionales de cada punto de carga, potencia instalada, factor de carga, y número de clientes de B.T, nos permitirán hallar los Índices de Calidad (descritos en el apartado 1.0.4).

\begin{tabular}{|cc|}
\hline Elemento & Tipo de Reposición \\
\hline SA00001 & reparación \\
IA00001 & reparación \\
LA10001 & reparación \\
XS00001 & reparación \\
LA10017 & reparación \\
FU00001 & reparación \\
TF50001 & reparación \\
LA10002 & maniobra \\
LA10003 & maniobra \\
XS00002 & maniobra \\
LA10004 & maniobra \\
XS00003 & maniobra \\
LA10010 & maniobra \\
XS00004 & maniobra \\
SV50001 & maniobra \\
LA10005 & maniobra \\
LA10006 & maniobra \\
XS00009 & maniobra \\
LA10007 & maniobra \\
AS00001 & maniobra \\
LA10008 & maniobra \\
XS00019 & maniobra \\
\hline
\end{tabular}

Fig.2.6.-Caminos Mínimos para el punto de carga número " 0 " de la red aérea.

Por tanto, debemos estudiar la manera de simular los ciclos de funcionamientofallo de un componente asociado a un evento en la red de fiabilidad de cada punto carga de una red de distribución.

Distinguiremos cuatro lógicas de funcionamiento diferentes para los distintos componentes de las redes que se han considerado en este estudio.

Lógica tipo 'a'. Elementos que tienen tasa de fallo nula y que, por tanto, nunca se considera su fallo. La secuencia del componente es de operación durante todo el periodo de simulación como se indica en la figura 2.7. Ejemplo de este tipo de elementos es la Salida de Subestación de Maniobra y/o Reparto (ver Anexo 1). 


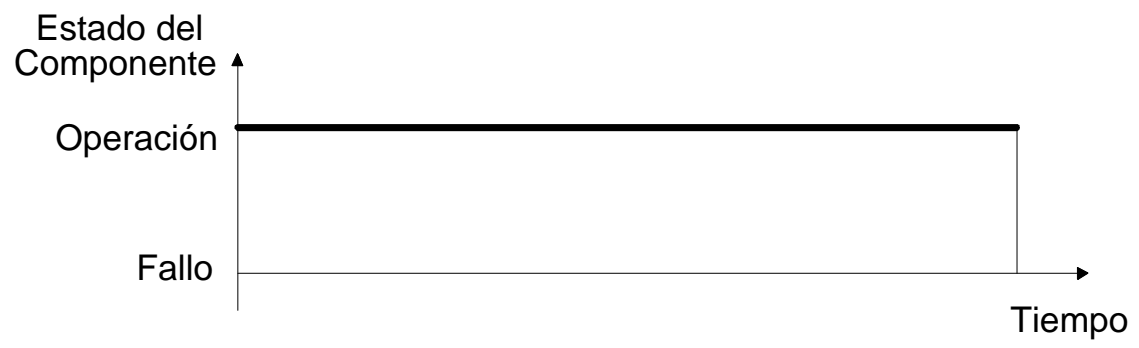

Fig.2.7.-Lógica de funcionamiento de elementos tipo 'a'

Lógica tipo 'b'. Elementos que no se ven afectados por las condiciones meteorológicas (elementos de interior). Poseen la misma tasa de reparación en buen y en mal tiempo y, además, la reposición del servicio puede realizarse indistintamente en uno u otro período. La simulación será muy sencilla. El ciclo básico constará de tres generaciones consecutivas:

1. Generar un número aleatorio uniforme entre 0 y 1 , para convertirlo, a continuación, a la distribución de probabilidad que hallamos determinado como la adecuada para describir el número de horas de funcionamiento o tiempo hasta el fallo.

2. Generar a continuación otro aleatorio, que corresponderá al número de horas de localización de la avería.

3. Generar un tercer número cuya transformación de lugar al tiempo de reparación de la avería.

La suma de estos dos últimos constituirá el tiempo total de fallo. Este ciclo de operación-fallo se repetirá hasta agotar el período de simulación como vemos en la figura 2.8 .

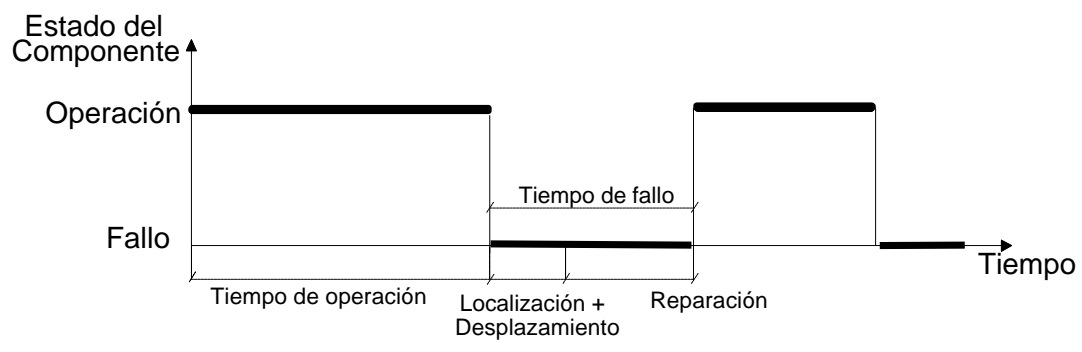

Fig.2.8.-Lógica de funcionamiento de elementos tipo 'b'

Lógica tipo 'c'. Elementos cuyo funcionamiento se ve influido por las condiciones meteorológicas, no siendo además posible la restauración del servicio durante los períodos de mal tiempo. Corresponde a elementos de intemperie, con modos de fallo, bien permanente, o bien por mantenimiento. La lógica de funcionamiento será la siguiente: 
1. Simular un ciclo de buen-mal tiempo considerando, como ya dijimos en este apartado, que las duraciones de ambos tipos de tiempo se distribuyen exponencialmente. Consideramos que la simulación comienza con un periodo de buen tiempo y con los componentes de la Red en funcionamiento ( si se deseara, podría variar esta circunstancia, aunque no influiría en el resultado).

2. Dentro de este ciclo de buen-mal tiempo simularemos ciclos funcionamientofallo, de la misma manera que los elementos de lógica 'b', hasta llegar al final de dicho ciclo meteorológico.

3. Cuando la simulación de los ciclos de operación alcancen el fín del ciclo meteorológico, volveremos a generar otro ciclo de buen-mal tiempo y sobre éste, una nueva serie de ciclos de operación. Este proceso se repetirá hasta que se supere el período de simulación deseado.

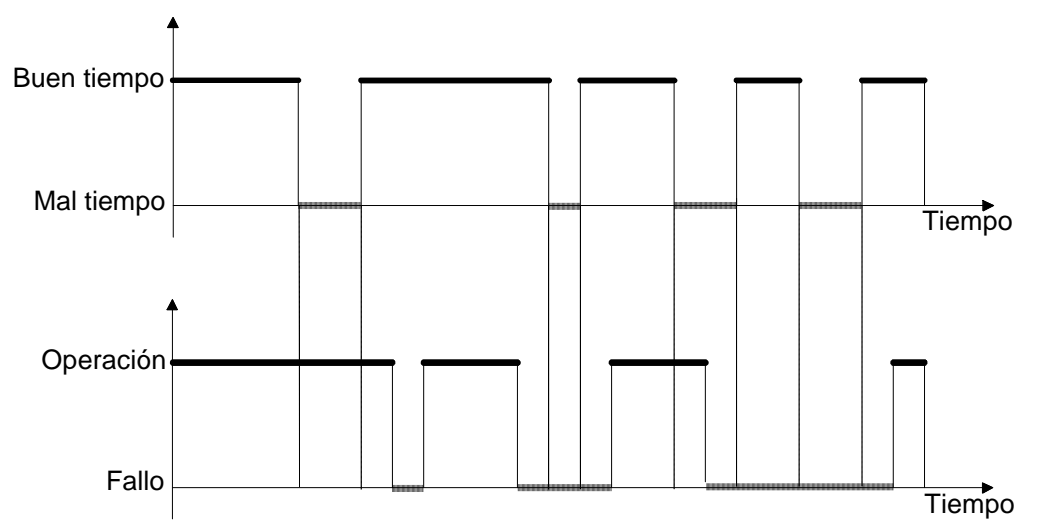

Fig.2.9.-Lógica de funcionamiento de elementos tipo 'c'

En la figura 2.9 se refleja la lógica de funcionamiento de los elementos tipo 'c'. Esta lógica, más complicada que las precedentes, precisa una descripción más detallada:

- Después de generar un periodo de operación, si el final de éste, o sea el fallo, se produce en un instante posterior al del final del período actual de buen tiempo, acumulamos el tiempo de operación hasta dicho final, y generamos un nuevo tiempo de operación en el periodo de mal tiempo; si esta generación supera el fin del periodo de mal tiempo, acumulamos este tiempo de operación con el anterior y, después de generar un nuevo ciclo de buen-mal tiempo, volvemos a generar un nuevo tiempo de operación en el nuevo periodo de buen tiempo. Este proceso se repetirá hasta que el periodo de operación no supere el punto de cambio de las condiciones meteorológicas (de buen a mal tiempo).

- Si después de haber generado un periodo de operación en un periodo de mal tiempo, la avería se produjera durante ese periodo de mal tiempo, consideraremos que las brigadas de reparación no comienzan su trabajo hasta que no finaliza el periodo de mal tiempo, con lo que el proceso de localización + desplazamiento + reparación se pospone hasta la llegada del buen tiempo, acumulándose este mal tiempo que restaba, al tiempo de fallo. 
- Si después de haber generado un periodo de operación en un periodo de buen tiempo, la avería se produjera durante ese periodo de buen tiempo, el proceso de reparación se llevaría a cabo. Si durante el proceso de reposición de servicio entramos en un periodo de mal tiempo, el proceso de reposición se paralizaría, y la duración de este período de mal tiempo se acumularía al tiempo de fallo. Si en el momento de transición a mal tiempo ya se hubiera localizado la avería, al reanudar los trabajos de reposición, la localización no hay que llevarla a cabo de nuevo, mientras que el proceso de reparación habrá que repetirlo desde el principio.

Lógica tipo 'd'. Elementos cuyo funcionamiento se ve influido por las condiciones meteorológicas, pero a diferencia de los elementos de lógica 'c', si es posible la restauración del servicio durante los períodos de mal tiempo. Esta lógica aparece en los eventos en los que la reposición del servicio se realiza por maniobra de un elemento telemandado. La lógica de funcionamiento será la siguiente:

1. Simular un ciclo de buen-mal tiempo de la misma manera que se ha descrito en la lógica anterior.

2. Dentro de este ciclo de buen-mal tiempo simularemos ciclos funcionamientofallo, de la misma manera que los elementos de lógica 'b', hasta llegar al final de dicho ciclo meteorológico.

3. Cuando la simulación de los ciclos de operación alcancen el fin del ciclo meteorológico, volveremos a generar otro ciclo de buen-mal tiempo y sobre éste, una nueva serie de ciclos de operación. Este proceso se repetirá hasta que se supere el período de simulación deseado.

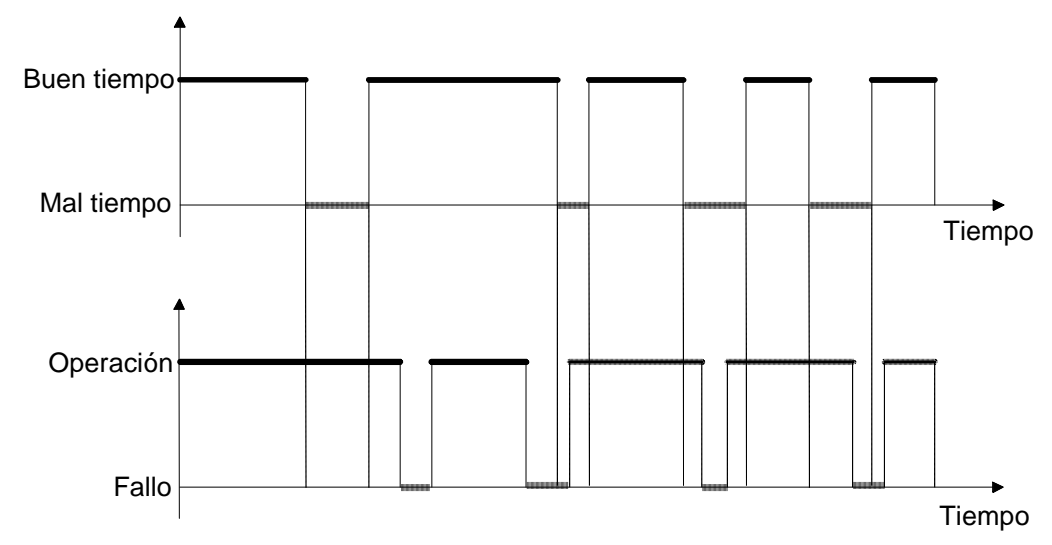

Fig.2.10.-Lógica de funcionamiento de elementos tipo 'd'

En la figura 2.10 se señala la lógica de funcionamiento de los elementos tipo 'd'. El funcionamiento de un elemento es igual que en la lógica precedente, salvo que los periodos de mal tiempo no influyen de ningún modo en el proceso de reposición de servicio.

\section{Mantenimiento en redes de Distribución}


Según sus objetivos podemos distinguir dos tipos de mantenimiento: el mantenimiento correctivo y el mantenimiento preventivo.

El mantenimiento correctivo consiste en la reposición de servicio, una vez producida una avería. Ya vimos en este apartado las incidencias que se podían producir en una red, sus causas, y las distintas formas de reponer el servicio.

El mantenimiento preventivo es el conjunto de actuaciones sobre los diversos componentes de la red, que tiene como objetivo fundamental mantener en buen estado de conservación dichos elementos, con el fin de evitar que el envejecimiento de los mismos provoque un aumento en su tasa media de fallo y por tanto un empeoramiento de la calidad de servicio. El mantenimiento preventivo permite, teóricamente, alargar indefinidamente la vida útil de un componente.

Podemos distinguir varios tipos de mantenimiento preventivo según sea periódico o aperiódico, se realice con tensión o sin ella. Sin embargo, desde el punto de vista del estudio de la fiabilidad, sólo interesa el mantenimiento preventivo sin tensión toda vez que provoca o puede provocar indisponibilidad. Éste se lleva a cabo desconectando el elemento sometido a mantenimiento, pudiendo provocar indisponibilidad programada en clientes si no hay posibilidad de alimentación alternativa durante la realización del mismo, pero también pudiendo provocar indisponibilidad imprevista.

El mantenimiento preventivo posee siempre carácter programado, y como tal, en su modo "sin tensión", contribuirá a los índices de calidad programados que no consideraremos en esta Tesis, como se señaló en el apartado 1.0.4. Sin embargo, dicho mantenimiento aporta también indisponibilidad a los índices de calidad no programados, desde el momento que el suceso que provoca dicha indisponibilidad está constituido simultáneamente por el componente sujeto a mantenimiento y por un componente de alimentación alternativa que falla de modo no programado. De este modo, se podría considerar la indisponibilidad imprevista provocada por el mantenimiento preventivo sin tensión debida a sucesos de segundo orden. En cualquier caso, dado que el $95 \%$ del mantenimiento se realiza con tensión, y por tanto no provoca indisponibilidad, y que cuando se realiza sin tensión se procura mantener varios elementos próximos a la vez, se obtiene que la aportación que pueden realizar estos eventos de segundo orden a los índices de calidad es muy pequeña, por lo que prescindiremos de ellos.

Así, el único aspecto bajo el que se contempla el mantenimiento preventivo (con o sin tensión) en esta Tesis, es la influencia que posee sobre las tasas de fallo de los componentes de la red. La hipótesis, ya formulada en este apartado, es que el mantenimiento realizado sobre los componentes de la red es suficientemente bueno como para que los componentes de la red se mantengan dentro de su vida útil, y por tanto que su tiempo hasta el fallo se distribuya según una exponencial negativa con una tasa de fallo constante. 


\subsection{LA HERRAMIENTA INFORMÁTICA}

La aplicación informática desarrollada para el análisis, desde el punto de vista de la fiabilidad, de diferentes sistemas de distribución de energía eléctrica en media tensión fue desarrollada en el lenguaje de programación 'C', siguiendo la norma ANSI para que pudiera ser fácilmente transportable a diferentes plataformas.

La aplicación consta de las etapas ejecutadas secuencialmente, que se muestran en la figura 2.11.

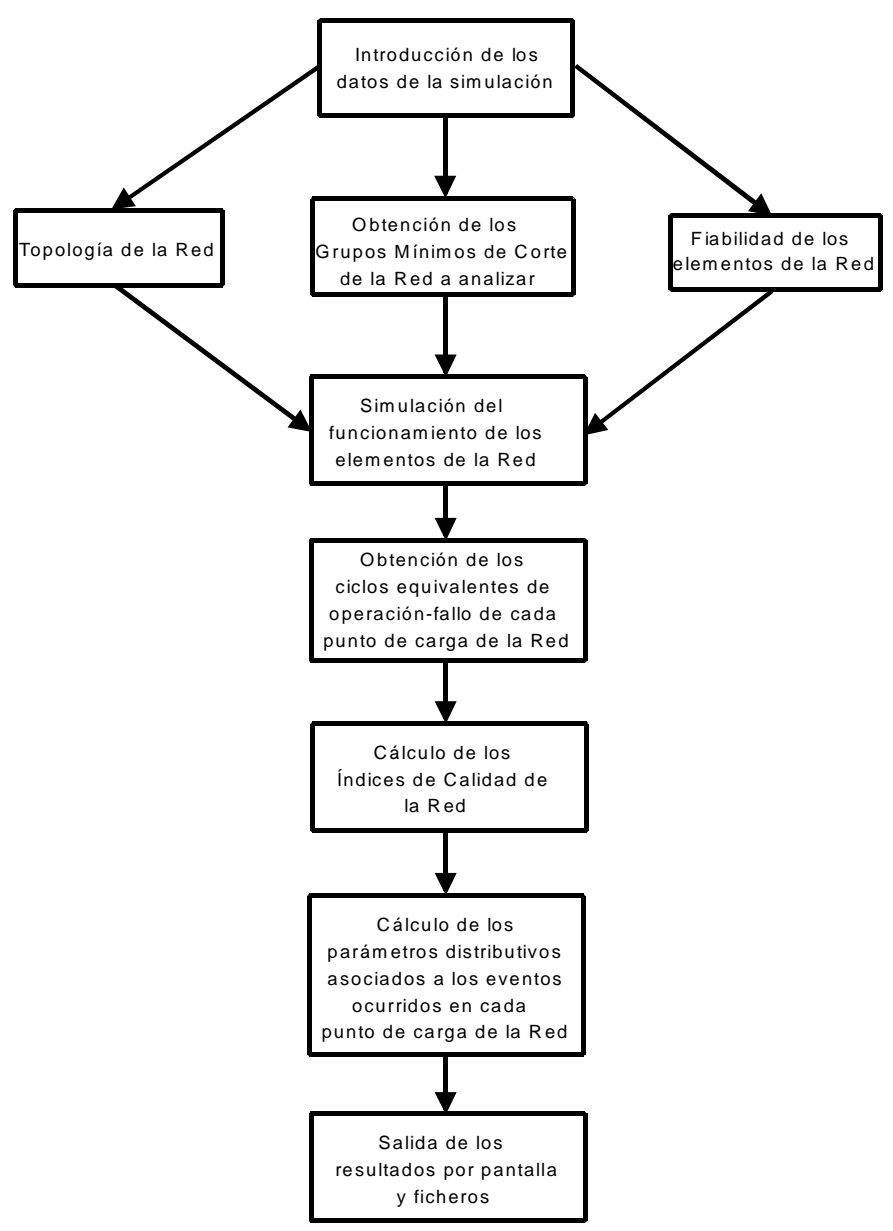

Fig.2.11.-Diagrama del funcionamiento del programa informático A continuación se describen más detalladamente las etapas de las que consta la aplicación informática y que podemos ver en la figura 2.11.

- Introducción de los datos de la simulación: Red a analizar, tiempo de simulación, número de simulaciones y tipo de distribuciones de los tiempos entre eventos de los elementos de la Red y condiciones meteorológicas.

- Obtención de los Grupos Mínimos de Corte de primer orden para fallos permanentes de cada uno de los punto de carga de la Red seleccionada. Esta etapa se encuentra desarrollada en un programa separado del resto ya que no es 
necesario obtener los mismos GMC de la red cada vez que simulemos. El resultado lo escribimos en un fichero.

- Almacenamiento de los datos de la red: leemos los ficheros donde consta la topología de la red bajo análisis (ver Anexo 3), los datos de fiabilidad de los componentes que forman esa red (ver Tabla A2-3 en el Anexo 2) y los grupos mínimos de corte de cada punto de carga obtenidos en la etapa anterior.

- Simulación de los ciclos de operación-fallo de cada uno de los elementos que forman parte de los GMC de cada punto de carga, a partir del módulo generador de números aleatorios.

- Obtención de las secuencias de operación-fallo de los puntos de carga existentes en la Red a analizar. Ya que los elementos que componen cada GMC están asociados en serie, compondremos sus respectivos ciclos de funcionamiento-fallo de forma que en los intervalos de tiempo en los que algún elemento del GMC se encuentre en fallo el punto de carga se halle fuera de servicio. Por tanto, para cada punto de carga de la Red sumaremos en serie los ciclos de operación-fallo de cada elemento que lo compone para obtener la secuencia equivalente de los ciclos operación-fallo de cada punto de carga.

- Cálculo de los Índices de Calidad de la red a partir de las secuencias equivalentes de ciclos de operación-fallo de cada punto de carga. Un ejemplo se muestra en la figura 2.12 .

- Cálculo de la indisponibilidad y $\mathbf{n}^{\mathbf{0}}$ de interrupciones medio por año y punto de carga de la red, a partir de las secuencias equivalentes de ciclos de operación-fallo de cada punto de carga. Un ejemplo se muestra en la figura 2.13 .

- Cálculo de los parámetros de la distribución estadística del tiempo hasta la interrupción y la de la duración de éstas, para cada punto carga (ver figura 2.14). Una vez obtenidos estos parámetros, el programa puede volver a simular el comportamiento de la red sin tener que simular el comportamiento de cada elemento, considerando cada punto de carga como un sólo elemento caracterizado por las distribuciones "Tiempo hasta la Interrupción" y "Tiempo de Indisponibilidad" que acabamos de obtener.

- Salida de resultados por pantalla y por ficheros para su almacenamiento y posterior utilización.

\begin{tabular}{l}
\hline DATOS DE LA SIMULACIÓN \\
Red $=$ aerea \\
Fich. de fiabilidad $=$ lib0 \\
Numero experimentos $=100$ \\
Tiempo medio de desplazamiento $=1.50$ \\
Tipo de distribución para el tiempo atmosférico = exponencial \\
Tipo de distribución para el tiempo hasta el fallo $=$ exponencial \\
Tipo de distribución para el tiempo de reparación = exponencial \\
Tipo de distribución para el tiempo de localización = exponencial \\
Tiempo de simulación $=2000000$ horas \\
\hline RESULTADOS
\end{tabular}




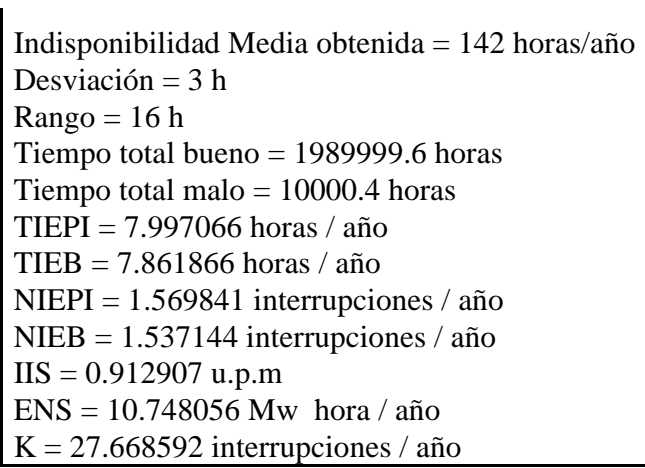

Fig.2.12.-Fichero de resultados típico, de la aplicación informática, que nos muestra los índices de calidad para la red aérea, junto con los parámetro usados en la simulación.

\begin{tabular}{|ccc|}
\hline Punto de carga & $\begin{array}{c}\text { Indisponibilidad } \\
\text { (horas por año) }\end{array}$ & $\begin{array}{c}\mathbf{N}^{\mathbf{0}} \text { interrupciones } \\
\text { (por año) }\end{array}$ \\
\hline 0 & 4.676570 & 1.122112 \\
1 & 5.001730 & 1.140946 \\
2 & 5.197989 & 1.133194 \\
3 & 7.379201 & 1.592393 \\
4 & 7.698900 & 1.601679 \\
5 & 7.883581 & 1.600277 \\
6 & 9.519443 & 1.810605 \\
7 & 5.886887 & 1.132186 \\
8 & 8.624732 & 1.625812 \\
9 & 9.062343 & 1.768513 \\
1 & 9.144156 & 1.737371 \\
1 & 10.89590 & 1.963116 \\
1 & 8.693955 & 1.670795 \\
1 & 8.938736 & 1.664181 \\
1 & 8.934297 & 1.668823 \\
1 & 8.656140 & 1.647274 \\
1 & 8.915577 & 1.638996 \\
1 & 6.403433 & 1.150319 \\
\hline
\end{tabular}

Fig.2.13.-Fichero de resultados típico, de la aplicación informática, que nos muestra la indisponibilidad y el número de interrupciones en cada punto de carga de la simulación de la figura 2.12.

\begin{tabular}{|c|c|c|c|c|c|c|c|c|}
\hline & \multicolumn{2}{|c|}{$\begin{array}{c}\text { Tasas de fallo }(\lambda=\text { buen } \\
\text { tiempo, } \mathbf{w}=\text { mal tiempo) }\end{array}$} & \multicolumn{5}{|c|}{$\begin{array}{c}\text { Duración de la reposición } \\
(\mathbf{r}=\text { buen tiempo, } \rho=\text { mal tiempo) }\end{array}$} \\
\cline { 2 - 9 } & \multicolumn{2}{|c|}{ Permanente } & \multicolumn{2}{|c|}{ Reparac./sustitución } & \multicolumn{2}{c|}{ Maniobra/acciona. } & \multicolumn{2}{c|}{ Pseudosustitución } \\
\hline \multirow{2}{*}{ Nombre } & $\lambda^{\mathrm{P}}$ & $\mathrm{W}^{\mathrm{P}}$ & $\mathrm{r}^{\mathrm{R}}$ & $\rho^{\mathrm{R}}$ & $\mathrm{r}^{\mathrm{A}}$ & $\rho^{\mathrm{A}}$ & $\mathrm{r}^{\mathrm{S}}$ & $\rho^{\mathrm{S}}$ \\
\hline P00 & 1.146709 & 1.146709 & 4.457362 & 4.457362 & -1 & -1 & -1 & -1 \\
\hline P01 & 1.159295 & 1.159295 & 4.638580 & 4.638580 & -1 & -1 & -1 & -1 \\
\hline P02 & 1.138924 & 1.138924 & 4.955849 & 4.955849 & -1 & -1 & -1 & -1 \\
\hline P03 & 1.616059 & 1.616059 & 4.840814 & 4.840814 & -1 & -1 & -1 & -1 \\
\hline P04 & 1.616274 & 1.616274 & 5.160932 & 5.160932 & -1 & -1 & -1 & -1 \\
\hline P05 & 1.609927 & 1.609927 & 5.246784 & 5.246784 & -1 & -1 & -1 & -1 \\
\hline P06 & 1.823127 & 1.823127 & 4.862928 & 4.862928 & -1 & -1 & -1 & -1 \\
\hline P07 & 1.150430 & 1.150430 & 5.285123 & 5.285123 & -1 & -1 & -1 & -1 \\
\hline
\end{tabular}


CAPÍTULO 2 - MODELO PROPUESTO

\begin{tabular}{|c|c|c|c|c|c|c|c|c|}
\hline P08 & 1.640526 & 1.640526 & 4.940722 & 4.940722 & -1 & -1 & -1 & -1 \\
\hline P09 & 1.807637 & 1.807637 & 5.452417 & 5.452417 & -1 & -1 & -1 & -1 \\
\hline P10 & 1.728942 & 1.728942 & 4.854571 & 4.854571 & -1 & -1 & -1 & -1 \\
\hline P11 & 1.984623 & 1.984623 & 5.273633 & 5.273633 & -1 & -1 & -1 & -1 \\
\hline P12 & 1.683165 & 1.683165 & 4.845668 & 4.845668 & -1 & -1 & -1 & -1 \\
\hline$\overline{P 13}$ & 1.646746 & 1.646746 & 4.996223 & 4.996223 & -1 & -1 & -1 & -1 \\
\hline P14 & 1.672462 & 1.672462 & 4.944518 & 4.944518 & -1 & -1 & -1 & -1 \\
\hline P15 & 1.700765 & 1.700765 & 4.961402 & 4.961402 & -1 & -1 & -1 & -1 \\
\hline P16 & 1.655060 & 1.655060 & 5.065953 & 5.065953 & -1 & -1 & -1 & -1 \\
\hline P17 & 1.153985 & 1.153985 & 5.714624 & 5.714624 & -1 & -1 & -1 & -1 \\
\hline
\end{tabular}

Fig.2.14.-Fichero de resultados típico, de la aplicación informática que nos muestra, ya en forma de fichero de fiabilidad para su posterior utilización en simulación, los parámetros de la distribución de los ciclos equivalentes de funcionamiento-fallo de cada punto de carga, de la simulación de la figura 2.12 . 



\section{ESCENARIOS DE APLICACIÓN}

\subsection{INTRODUCCIÓN}

Las redes eléctricas tienen como misión la transmisión de la energía eléctrica desde los centros de producción hasta los usuarios de la misma. Desde un punto de vista estructural, cada una de las etapas intermedias que aparece en la transmisión posee características diferentes. Así, podemos distinguir tres niveles en el sistema de transmisión de energía:

-. Nivel de Distribución (media y baja tensión)

-. Nivel de Reparto

-. Nivel de Transporte

Lo que se denomina comúnmente como Sistema de Distribución, está compuesto por las Estaciones Transformadoras de Distribución, las Redes de Distribución, los Centros de Transformación, las redes secundarias o de baja tensión $\mathrm{y}$, finalmente, las conexiones a clientes.

\section{Estructuras en Redes de Distribución}

Las distintas características de las diversas zonas de consumo de energía eléctrica obligan a adoptar soluciones técnicas diferentes (M_CAL-96), dando lugar a la consideración de tres tipos genéricos de Redes de Distribución en Media Tensión, según aparece recogido en la Tabla 3.1. 


\begin{tabular}{|l|l|l|}
\hline Tipo de red & Estructura topológica & Forma de explotación \\
\hline Aérea & radial & radial \\
\hline Subterránea & mallada & radial \\
\hline Mixta & radial/mallada & radial \\
\hline
\end{tabular}

Tabla 3.1.- Clasificación convencional de las redes de distribución

Análogamente, las distintas zonas de consumo podemos clasificarlas de acuerdo a la Tabla 3.2.

\begin{tabular}{|l|l|}
\hline Zona & Población \\
\hline Urbana & mayor de 50000 habitantes \\
\hline Semirural & entre 5000 y 50000 habitantes \\
\hline Rural & menor de 5000 habitantes \\
\hline
\end{tabular}

Tabla 3.2.- Zonas de consumo

Describiremos, sucintamente, las peculiaridades de la estructura de cada uno de los tres tipos genéricos de redes de distribución enunciados.

- Red Aérea de Media Tensión.

Su estructura topológica es de tipo radial, disponiendo, con frecuencia, de redundancia "standby", esto es, elementos de maniobra normalmente abiertos, que permiten, al ser cerrados, establecer circuitos de alimentación alternativos cuando por alguna contingencia no puede ser utilizado el circuito que inicialmente alimentaba a una zona determinada de la red.

La estructura radial proviene del hecho de que la red se construye con una estructura arborescente, con una línea principal o alimentador que parte de la Subestación de Transformación y/o Maniobra y de la que derivan ramas principales, de las que, a su vez, derivan ramas secundarias, pudiendo alcanzar varios niveles de derivación.

Debe hacerse notar que, aunque la estructura de las redes aéreas permita la posibilidad de cerrar bucles alrededor de una Subestación de Transformación y/o Maniobra ó de crear mallas en las que confluyan, de forma simultánea, varias alimentaciones, su esquema de explotación es siempre radial.

- Red Subterránea de Media Tensión.

$\mathrm{Su}$ estructura se establece, en general, de forma mallada. Se dispone así de la posibilidad de cierre en bucle, tanto en los propios centros como en toda la red, para atender determinadas exigencias de explotación, como la necesidad de disponer de alimentaciones alternativas ocasionada por averías en componentes, mantenimientos programados, etc.

Al igual que en el caso de las redes aéreas, debe tenerse en cuenta que, aunque la estructura de las redes subterráneas permita cerrar bucles o crear 
mallas en las que confluyan de forma simultánea varias alimentaciones, el esquema de explotación de estas redes se establece siempre en forma radial.

- Red Mixta de Media Tensión.

Catalogaremos una red de distribución de Media Tensión como red mixta, cuando en ella coexistan tramos de red aérea y de red subterránea de longitud significativa. Cada uno de los tramos de este tipo de red participará de la estructura característica de red aérea o subterránea, respectivamente. El esquema de explotación será siempre radial.

\subsection{REDES DE ENSAYO}

Los esquemas topológicos de las redes que hemos diseñado para la obtención y verificación, en su caso, de resultados en esta Tesis, se representan en las figuras 3.1, 3.2 y 3.3. A fin de poder aplicar las técnicas de simulación propuestas a diversos tipos de redes y poder separar, en su caso, el efecto de las condiciones meteorológicas sobre el comportamiento de éstas, elegimos, esencialmente, tres tipos diferentes de redes de distribución, una red aérea, una red subterránea y una red mixta. En las tasas de fallo de los elementos de la red subterránea no influyen las condiciones meteorológicas, sin embargo, las tasas de fallo de las líneas aéreas se verán afectadas por las condiciones meteorológicas modificando la indisponibilidad de la red aérea y la red mixta.

En las figuras 3.1, 3.2 y 3.3 se señalan los componentes que constituyen las dos redes elegidas; las dos primeras letras de la abreviatura indican el tipo del componente y el número que las sigue indica su número de orden elegido arbitrariamente. Para conocer el subtipo que corresponde a cada uno de los componentes que podemos encontrar en estas figuras deberemos consultar las tablas de datos topológicos de los componentes, en el Anexo 3. Deberemos identificar, en la tabla, las dos primeras letras del componente buscado, y su número de orden con las últimas cifras que nos encontramos en cada nombre. Una vez encontrado, el primer número que sigue a las dos letras nos indicará el subtipo del que se trata. Una descripción detallada de los componentes considerados en estas redes, así como sus características de fiabilidad, se hallan recogidas en los Anexos 1 y 2.

La red de ensayo de la figura 3.1 posee la estructura topológica típica de una red de distribución aérea, y ha sido extraída de la red de distribución aérea rural Villafuerte-Peñafiel. Se han destacado aquellos componentes de cuyo fallo o mal funcionamiento puede derivarse un cierto grado de indisponibilidad.

La red de ensayo de la figura 3.2 posee la estructura topológica típica de una red de distribución subterránea y se ha inspirado en una fracción de la red de distribución subterránea de Valencia capital. Se han destacado aquellos componentes de cuyo fallo o mal funcionamiento puede derivarse un cierto grado de indisponibilidad. 


\section{RED DE ENSAYO AÉREA}

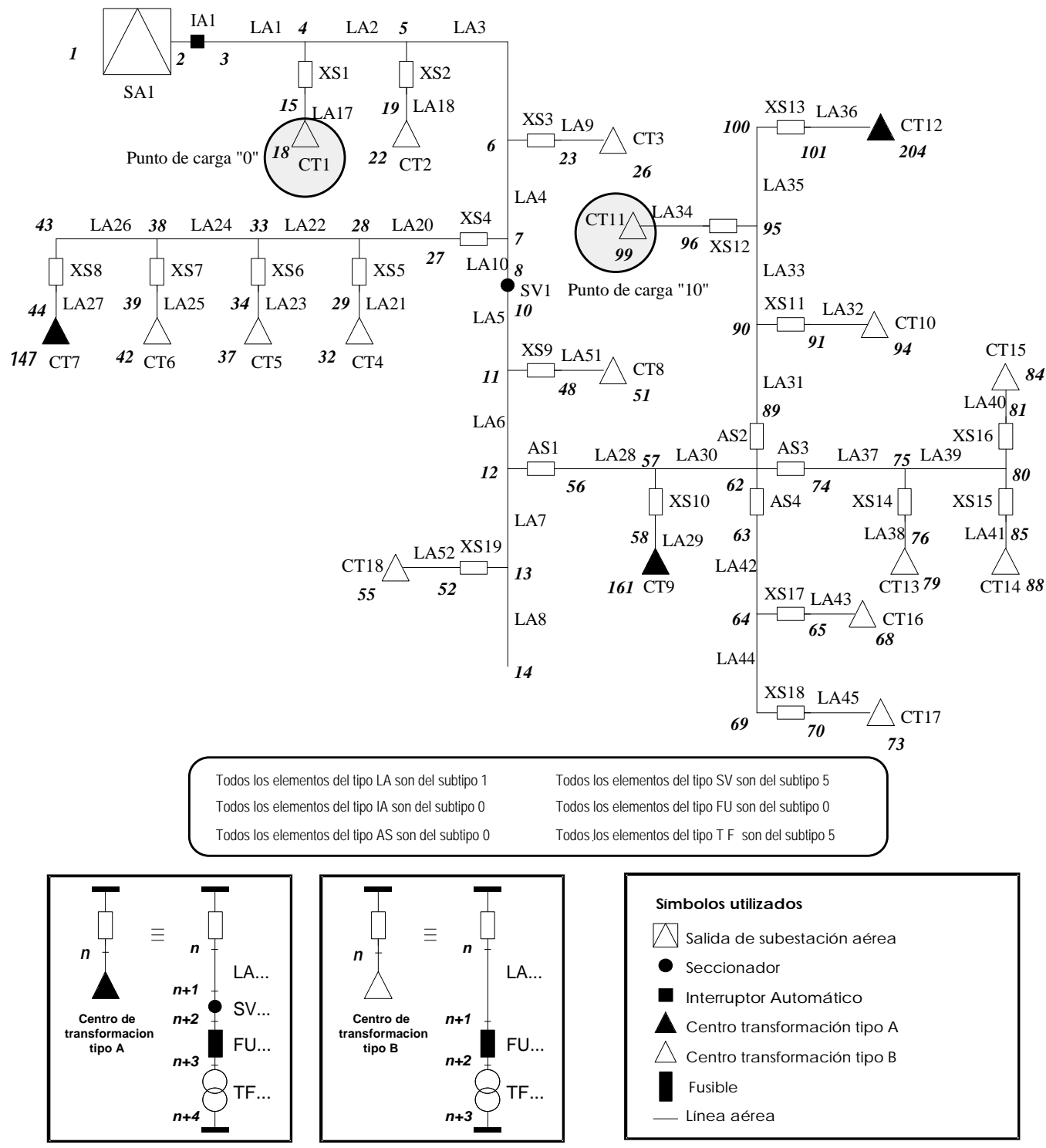

Fig.3.1.-Red de ensayo aérea. Denominaciones en Anexo 1. Valores de parámetros de fiabilidad en Anexo 2. Datos topológicos e identificación de subtipos en Anexo 3. 


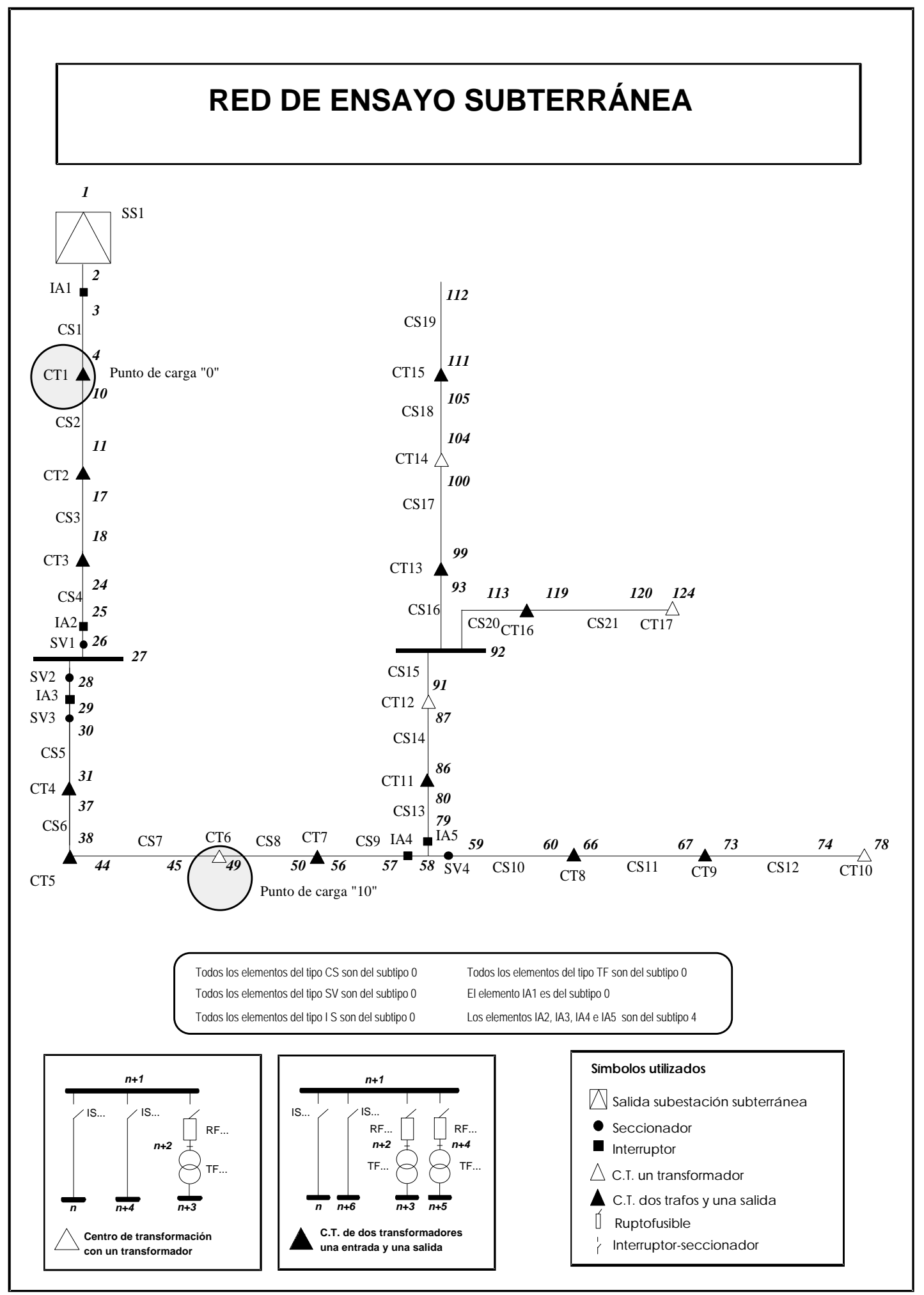

Fig.3.2.-Red de ensayo subterránea. Denominaciones en Anexo 1. Valores de parámetros de fiabilidad en Anexo 2. Datos topológicos e identificación de subtipos en Anexo 3. 


\section{RED DE ENSAYO MIXTA}

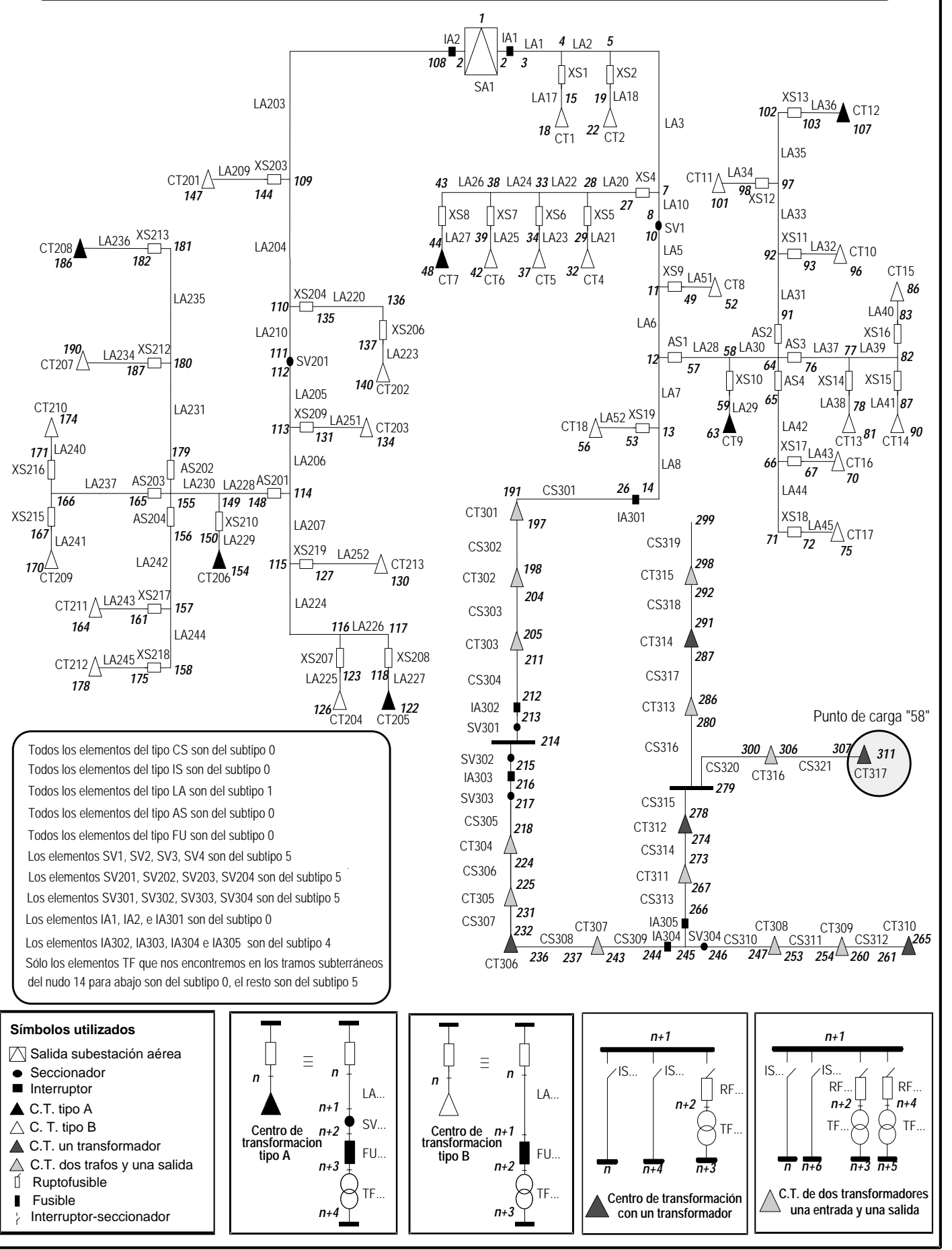

Fig.3.3.-Red de ensayo mixta Denominaciones en Anexo 1. Valores de parámetros de fiabilidad en Anexo 2. Datos topológicos e identificación de subtipos en Anexo 3.

La red de ensayo de la figura 3.3 se ha construido uniendo fragmentos de las dos redes anteriores. 


\subsubsection{Escenarios para la obtención de Índices de Calidad de Servicio}

Se han seleccionado un conjunto de situaciones, como representativas, que nos permitirán analizar, en esta Tesis, la influencia de las distribuciones de probabilidad de los diferentes tiempos entre eventos, en los índices de calidad (definidos en el apartado 1.0.4) de las tres redes de ensayo. Analizaremos los siguientes casos:

- Influencia del tipo de distribución elegida para el tiempo de reparación de los elementos que componen la red. Modelización realista de algunos sucesos.

- Influencia de la duración media del tiempo de localización y desplazamiento asociado a las interrupciones de suministro. Acciones de explotación.

- Influencia de las duraciones medias de los ciclos de buen y mal tiempo, $N$ y $S$ respectivamente.

- Influencia relativa de los distintos elementos que componen cada red en sus índices de calidad. Esta influencia la estudiaremos a partir de las tasa de fallo y tiempos de reparación de los elementos. Permite examinar acciones de Planificación y Mantenimiento.

Cada conjunto de situaciones constituye un escenario de aplicación de los experimentos de simulación. La definición de situaciones se hace, en cualquier caso, determinando el conjunto de características de las distribuciones de probabilidad de los diferentes tiempos entre eventos que determinan el fucionamiento de la red. Para todos estos escenarios se han elegido los siguientes parámetros para los experimentos de simulación:

- Tiempo de simulación en la red subterránea: 2.000.000 horas

- Tiempo de simulación en la red subterránea: 200.000 horas

- Tiempo de simulación en la red subterránea: 200.000 horas

- Numero de experimentos en cada simulación: 100

- Escenario CAL-1: En el que evaluaremos la influencia del tipo de distribución elegida para el tiempo de reparación de los elementos que componen las redes que se han diseñado, comparando, también, los resultados, producto de nuestra simulación, con los obtenidos mediante procesos de Markov, sabiendo que en este método, todos los tiempos entre eventos se consideran distribuidos exponencialmente.

Se utilizan, como características de fiabilidad base de los elementos de la red, los valores de las tasas de fallo y tiempos de reparación que aparecen en el Anexo 2. Los valores correspondientes a las líneas aéreas del subtipo (LA1) y los cables subterráneos del subtipo (CSO) se decrementarán e incrementarán para disponer de dos pruebas más con las que afinar nuestro criterio de valoración. Se han elegido los elementos CS0 y LA1 por ser los que mayor influencia tienen en la indisponibilidad de sus redes respectivas, como podremos ver en el escenario CAL-4. Las condiciones que impondremos a nuestra simulación serán las de la Tabla 3.3. 


\begin{tabular}{|l|c|c|}
\cline { 2 - 3 } \multicolumn{1}{c|}{} & $\begin{array}{c}\text { Distribución de } \\
\text { probabilidad }\end{array}$ & $\begin{array}{c}\text { Duraciones medias } \\
\text { (horas). }\end{array}$ \\
\hline Ciclos de buen y mal de tiempo & exponencial & $\mathrm{N}=400 ; \mathrm{S}=2$ \\
\hline Tiempo hasta el fallo del componente & exponencial & $\begin{array}{c}\text { LA1 y CS0 } \\
\text { Caso base x 0,25 - 1 - 10 } \\
\text { Resto elementos: Anexo 2 }\end{array}$ \\
\hline Tiempo de desplazamiento y localización del fallo & exponencial & 1,5 \\
\hline & $\begin{array}{c}\text { exponencial y } \\
\text { normal }\end{array}$ & $\begin{array}{c}\text { LA1 y CS0 } \\
\text { Caso base x 0,25 - 1 - 10 } \\
\text { Resto elementos: Anexo 2 }\end{array}$ \\
\hline
\end{tabular}

Tabla 3.3. Condiciones de simulación, escenario CAL-1

Como se aprecia en la tabla anterior, para cada una de los tres conjuntos de parámetros de fiabilidad que empleamos, utilizaremos dos hipótesis para la distribución del tiempo de reparación de los componentes. Una será la exponencial, como en la técnica basada en procesos de Markov, y otra será la normal, que podemos considerar más razonable que la hipótesis anterior debido al carácter causal de la reparación de un componente, cuya duración tomará valores alrededor de una media con una mayor o menor dispersión.

- Escenario CAL-2: En el que evaluaremos la influencia de la duración media del tiempo de localización y desplazamiento asociado a las interrupciones de suministro.

Se utilizan, como características de fiabilidad de los elementos de la red, los valores de las tasas de fallo y tiempos de reparación que aparecen en el Anexo 2. Las condiciones que impondremos a nuestra simulación figuran en la Tabla 3.4.

\begin{tabular}{|c|c|c|}
\hline & $\begin{array}{c}\text { Distribución de } \\
\text { probabilidad }\end{array}$ & $\begin{array}{c}\text { Duraciones medias } \\
\text { (horas). }\end{array}$ \\
\hline Ciclos de buen y mal de tiempo & exponencial & $\mathrm{N}=400-\mathrm{S}=2$ \\
\hline Tiempo hasta el fallo del componente & exponencial & Anexo 2 \\
\hline Tiempo de desplazamiento y localización del fallo & exponencial & $\begin{array}{l}0-0,5-1-1,5 \\
2-3-4-5 \\
\end{array}$ \\
\hline Tiempo de reparación de un componente & exponencial & Anexo 2 \\
\hline
\end{tabular}

Tabla 3.4. Condiciones de simulación, escenario CAL-2

Utilizaremos ocho valores distintos para la duración media del tiempo de desplazamiento y localización con el fin de obtener las variaciones que esta variable produce en los índices de calidad de la red.

- Escenario CAL-3: En el que evaluaremos la influencia de las duraciones medias de los ciclos de buen y mal tiempo, $N$ y $S$ respectivamente. 
Como en el anterior escenario, se utilizan, como características de fiabilidad de los elementos de la red, los valores de las tasas de fallo y tiempos de reparación que aparecen en el Anexo 2. Las condiciones que impondremos a nuestra simulación figuran en la Tabla 3.5.

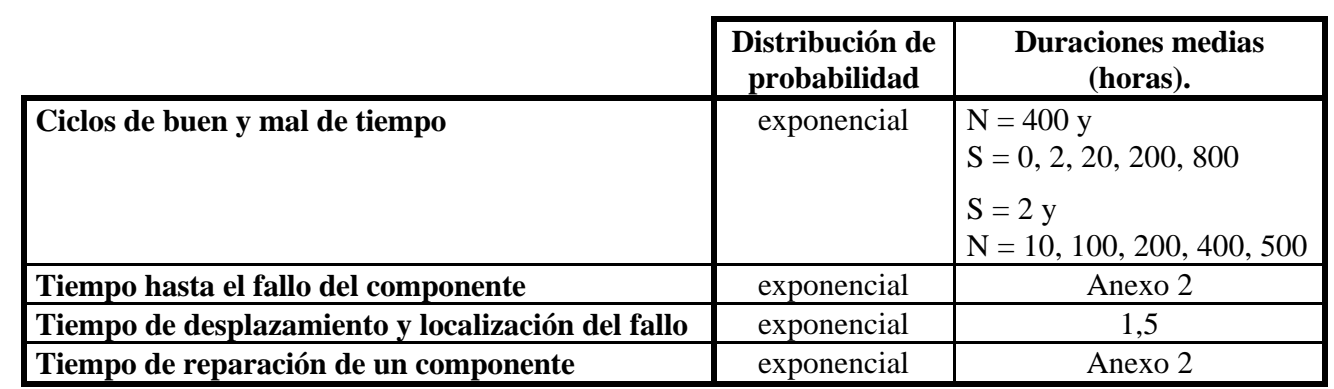

Tabla 3.5. Condiciones de simulación, escenario CAL-3

Utilizaremos siete combinaciones distintas para las duraciones medias de los ciclos de buen y mal tiempo con el fin de obtener las variaciones que esta variable produce en los índices de calidad de la red.

- Escenario CAL-4: En el que evaluaremos la influencia relativa de los distintos elementos que componen cada red en sus índices de calidad. Este estudio sensitivo se realizará evaluando la influencia que la modificación de las tasa de fallo y tiempos de reparación de los componentes más significativos (líneas aéreas, cables subterráneos, interruptores automáticos, seccionadores, seccionadores-fusibles, ruptofusibles y transformadores) ejerce sobre los índices de Calidad de Servicio.

Se utilizan, como características de fiabilidad base de los elementos de la red, los valores de las tasas de fallo y tiempos de reparación que aparecen en el Anexo 2. Los valores de cada elemento se decrementarán e incrementarán de forma individual para analizar las variaciones que provocan en los índices de calidad de la red. Las condiciones que impondremos a nuestra simulación figuran en la Tabla 3.6.

\begin{tabular}{|c|c|c|}
\hline & $\begin{array}{c}\text { Distribución de } \\
\text { probabilidad }\end{array}$ & $\begin{array}{c}\text { Duraciones medias } \\
\text { (horas). }\end{array}$ \\
\hline Ciclos de buen y mal de tiempo & exponencial & $\mathrm{N}=400$ y $\mathrm{S}=2$ \\
\hline Tiempo hasta el fallo del componente & exponencial & $\begin{array}{r}\text { Caso base } \times \begin{array}{r}0,25-0,5 \\
1-2-4\end{array} \\
\end{array}$ \\
\hline Tiempo de desplazamiento y localización del fallo & exponencial & 1,5 \\
\hline Tiempo de reparación de un componente & exponencial & $\begin{array}{r}\text { Caso base x } 0,25-0,5 \\
1-2-4\end{array}$ \\
\hline
\end{tabular}

Tabla 3.6. Condiciones de simulación, escenario CAL-4

Para poder obtener la influencia de cada componente en los índices de calidad de la red, variaremos conjuntamente los valores de las tasas de fallo y tiempos de reparación de cada componente, obteniendo cinco grupos de valores distintos para cada una de las tres redes de ensayo. 


\subsubsection{Escenarios para el análisis del comportamiento de las distribuciones del tiempo hasta la interrupción y tiempo de indisponibilidad}

Se han seleccionado un conjunto de situaciones, como representativas, que nos permitirán analizar el comportamiento de las distribuciones del tiempo hasta la interrupción y tiempo de indisponibilidad de los puntos de carga de las tres redes de ensayo diseñadas en esta Tesis. Cada conjunto de situaciones constituye un escenario de aplicación de los experimentos de simulación. La definición de situaciones se realiza, en cualquier caso, por modificación de las características de fiabilidad de los elementos que componen la red, y/o de las condiciones meteorológicas. Se eligen, en general puntos de carga significativos (marcados con un circulo sombreado en las figuras $3.1,3.2$ y 3.3 )

Para todos estos escenarios se han elegido los siguientes parámetros para los experimentos de simulación:

- Tiempo de simulación en la red subterránea: 2.000.000 horas

- Tiempo de simulación en la red subterránea: 200.000 horas

- Tiempo de simulación en la red subterránea: 200.000 horas

- Numero de experimentos en cada simulación: 100

\section{Escenarios para la red subterránea}

En el Anexo 2 se relacionan las tasa de fallo y tiempos de reparación asociados a los elementos que forman la red subterránea. Al tratarse de una red subterránea, el tiempo meteorológico no influirá en el comportamiento de sus elementos y por tanto no necesitamos incluirlo como variable en los escenarios que hemos diseñado. Nos centraremos exclusivamente en el análisis que la variación de la tasa de fallo y tiempos de reparación de uno o dos elementos de la red produce sobre su indisponibilidad, definiendo cuatro escenarios diferentes en los que combinar estas variaciones.

Los escenarios se utilizarán suponiendo que la hipótesis de distribución de los tiempos de reparación de los elementos de la red sigue, indistintamente, una distribución exponencial (DETR) o una normal truncada (DNTR).

- Escenario SUB-1: Se utilizan, como características de fiabilidad de los elementos de la red, los valores de las tasas de fallo y tiempos de reparación que aparecen en el Anexo 2. Considerando que estos valores podrían ser los usuales en una red real. A este caso le denominaremos, por ello, básico.

- Escenario SUB-2: Aumentamos, respecto del escenario básico, la tasa de fallo de uno o dos elementos. Los elementos elegidos son el cable subterráneo del subtipo 0, denominado CS0, y el interruptor automático del subtipo 4, denominado IA4 (ver Anexo 1). CS0 se elige por su gran abundancia dentro de la red (todos los cables subterráneos existentes son de este subtipo), porque su tasa de fallo es de las más elevadas y porque su tiempo medio de reparación es 
un orden por encima del resto (ver Anexo 2), siendo el elemento que más contribuye a la indisponibilidad total de la red. Todo ello hace suponer que una variación de sus características de fiabilidad influirá en gran medida en la indisponibilidad de la red y será fácilmente reconocible. Elegimos, además, otro componente, como IA4, con una tasa de fallo y un tiempo medio de reparación menores que los de CS0 que no es tan abundante como él, pero buscando que la variación de sus características de fiabilidad puedan influir de forma perceptible en la indisponibilidad de la red.

- Escenario SUB-3: En este escenario aumentamos, respecto del escenario básico, el tiempo de reparación de uno o dos elementos. Los elementos elegidos son los mismos que en el caso anterior, el cable subterráneo denominado CS0 y el interruptor automático denominado IA4, y por las mismas razones que en el caso anterior.

- Escenario SUB-4: En este escenario incrementamos, respecto del escenario básico, conjuntamente la tasa de fallo y el tiempo de reparación de CS0 e IA4.

\section{Escenarios para la red aérea}

Estudiaremos el efecto de las condiciones meteorológicas en la indisponibilidad de la red, sin modificar las características de fiabilidad de sus elementos. Como se expuso en el apartado 2.3, las condiciones meteorológicas se clasifican en dos tipos, tiempo bueno y tiempo malo, caracterizándose sus distribuciones de probabilidad por sendas exponenciales cuyas medias son $\mathrm{N}$, para el buen tiempo, y S, para el mal tiempo.

En la indisponibilidad de las redes aéreas, a diferencia de lo que sucede en las subterráneas, influyen las condiciones meteorológicas, porque condiciona el ciclo funcionamiento-fallo de alguno o todos sus elementos. En nuestro caso, sólo las líneas aéreas ven sus características de fiabilidad modificadas, poseyendo, durante el mal tiempo, una tasa de fallo 200 veces superior al que poseían en buen tiempo. De la misma manera, poseen un tiempo de reparación total igual a la suma del que tenía en buen tiempo y lo que resta de mal tiempo una vez que han fallado, ya que las líneas aéreas no se reparan hasta que no cesa el mal tiempo (ver Anexo 2).

Al igual que en la red subterránea, los escenarios que se consideran se utilizarán suponiendo que la hipótesis de distribución de los tiempos de reparación de los elementos de la red sigue, indistintamente, una distribución exponencial o una normal truncada.

- Escenario AER-1: A partir de un valor de 400 horas para la duración media de los ciclos de buen tiempo, $N$, variaremos la duración media de los ciclos de mal tiempo, $S$, para estudiar las repercusiones que ello provoca en la indisponibilidad de la red. Utilizaremos valores de S de 0, 2, 20 ,200 y $800 \mathrm{~h}$. 
- Escenario AER-2: Fijaremos aquí el valor de la duración media de los ciclos de mal tiempo (S) en 2 horas, y variaremos la duración media de los ciclos de buen tiempo (N) para estudiar los efectos que ello provoca en la indisponibilidad de la red. Utilizaremos valores de $\mathrm{N}$ de 10 y 100 horas.

\section{Escenarios para la red mixta}

Al igual que en la red aérea, estudiaremos el efecto de las condiciones meteorológicas en la indisponibilidad de la red sin modificar las características de fiabilidad de sus elementos.

Una red mixta consta de una o varias subredes subterráneas y de una o varias subredes aéreas interconectadas. En la indisponibilidad de los puntos carga abastecidos a través de redes mixtas influyen las condiciones meteorológicas, como consecuencia de los tramos aéreos. La diferencia entre estos puntos carga abastecidos a través de sistemas mixtos con los de la red aérea, es que, en aquellos, existirá una mayor proporción de elementos cuyas características de fiabilidad no se ven influidas por las condiciones meteorológicas.

El estudio de aquellos puntos carga en los que el suministro no dependa de ningún tramo aéreo es equivalente al realizado para la red subterránea. Por ello, únicamente estudiaremos puntos carga en los que el suministro dependa de algún tramo aéreo.

Al igual que en las dos redes anteriores, los escenarios que se consideran se utilizarán suponiendo que la hipótesis de distribución de los tiempos de reparación de los elementos de la red sigue, bien sea una distribución exponencial o una normal truncada.

- Escenario MIX-1: A partir de un valor de 400 horas como duración media de los ciclos de buen tiempo $(\mathrm{N})$, variaremos la duración media de los ciclos de mal tiempo (S) para estudiar las repercusiones que ello provoca en la indisponibilidad de la red. Utilizaremos valores de S de 0, 2, 20 ,200 y 800 horas.

- Escenario MIX-2: Fijaremos aquí un valor de la duración media de los ciclos de mal tiempo (S) en 2 horas, y variaremos la duración media de los ciclos de buen tiempo $(\mathrm{N})$ para estudiar los efectos que ello provoca en la indisponibilidad de la red. Utilizaremos valores de $\mathrm{N}$ de 10 y 100 horas. 


\subsection{GENERADORES DE VARIABLES ALEATORIAS. RESULTADOS DE LAS PRUEBAS ESTADÍSTICAS}

Las pruebas estadísticas, que publicamos en (A_FRE-ALA-94), se llevaron a cabo generando 1.000 secuencias diferentes para cada generador, con tres longitudes de secuencia diferentes, una de 10.000 números otra de 100.000 y una última de 1.000.000, ensayando en cada secuencia las pruebas estadísticas que describimos en el apartado 2.1. En las Tablas 4.1, 4.2 y 4.3 se indica el número de secuencias que no superaron alguna de estas pruebas, para los ocho generadores de números aleatorios seleccionados.

\begin{tabular}{|c|c|c|c|c|c|c|}
\cline { 2 - 7 } \multicolumn{1}{c|}{} & Promedio & Frecuencias & Distancias & S. Cuadradas & S. Cúbicas & Acotadas \\
\hline ran0 & 61 & 57 & 69 & 69 & 79 & 81 \\
\hline ran1 & 45 & 49 & 72 & 41 & 78 & 101 \\
\hline ran2 & 33 & 52 & 70 & 62 & 86 & 90 \\
\hline ran3 & 51 & 54 & 79 & 66 & 73 & 93 \\
\hline ran4 & 48 & 57 & 62 & 66 & 66 & 97 \\
\hline ran6 & 40 & 52 & 37 & 54 & 70 & 46 \\
\hline rand & 57 & 53 & 49 & 73 & 71 & 94 \\
\hline drand48 & 51 & 53 & 62 & 69 & 84 & 81 \\
\hline
\end{tabular}

Tabla 4.1.-Mil secuencias de 10.000 números aleatorios, con diversos generadores. Número de secuencias que no superan las pruebas correspondientes. 


\begin{tabular}{|c|c|c|c|c|c|c|}
\cline { 2 - 7 } \multicolumn{1}{c|}{} & Promedio & Frecuencias & Distancias & S. Cuadradas & S. Cúbicas & Acotadas \\
\hline $\operatorname{ran0}$ & 46 & 56 & 68 & 62 & 72 & 110 \\
\hline $\operatorname{ran1}$ & 42 & 45 & 66 & 58 & 73 & 82 \\
\hline $\operatorname{ran2}$ & 43 & 56 & 65 & 66 & 73 & 91 \\
\hline $\operatorname{ran3}$ & 54 & 39 & 59 & 53 & 85 & 80 \\
\hline $\operatorname{ran4}$ & 51 & 57 & 71 & 71 & 99 & 87 \\
\hline $\operatorname{ran6}$ & 40 & 52 & 37 & 54 & 70 & 46 \\
\hline rand & 51 & 57 & 71 & 71 & 99 & 87 \\
\hline drand48 & 63 & 58 & 62 & 82 & 89 & 78 \\
\hline
\end{tabular}

Tabla 4.2.-Mil secuencias de 100.000 números aleatorios, con diversos generadores. Número de secuencias que no superan las pruebas correspondientes.

\begin{tabular}{|c|c|c|c|c|c|c|}
\cline { 2 - 7 } \multicolumn{1}{c|}{} & Promedio & Frecuencias & Distancias & S. Cuadradas & S. Cúbicas & Acotadas \\
\hline $\operatorname{ran0}$ & 50 & 48 & 63 & 52 & 96 & 89 \\
\hline $\operatorname{ran1}$ & 39 & 49 & 81 & 59 & 80 & 85 \\
\hline $\operatorname{ran2}$ & 52 & 60 & 78 & 62 & 81 & 78 \\
\hline $\operatorname{ran3}$ & 47 & 52 & 56 & 76 & 124 & 94 \\
\hline $\operatorname{ran4}$ & 40 & 45 & 81 & 52 & 69 & 79 \\
\hline $\operatorname{ran6}$ & 41 & 43 & 70 & 66 & 79 & 83 \\
\hline rand & 63 & 58 & 62 & 82 & 89 & 78 \\
\hline drand48 & 45 & 50 & 72 & 72 & 85 & 87 \\
\hline
\end{tabular}

Tabla 4.3.-Mil secuencias de 1.000.000 números aleatorios, con diversos generadores. Número de secuencias que no superan las pruebas correspondientes.

Los resultados muestran que no hay diferencia significativa en utilizar uno $\mathrm{u}$ otro generador en ninguna de las tres longitudes de secuencia empleadas. En ningún caso llega al $10 \%$ el número de secuencias que no supera cada test. Sin embargo, el generador denominado ran6( ) es el que mejores resultados obtuvo para períodos cortos como son los empleados en esta Tesis.

\section{Otras consideraciones sobre los generadores de variables aleatorias uniformes.}

Por último, antes de determinar la elección del generador que utilizaremos para nuestros experimentos de simulación, además de la influencia de los test de aleatoriedad y uniformidad, tendremos en cuenta, en primer término, el período que vamos a necesitar, y en segundo la sencillez y rapidez del generador, así como su portabilidad. 
Realizamos una prueba para medir el tiempo de cómputo empleado por cada uno de los distintos algoritmos generadores de números aleatorios en producir una muestra de números de tamaño genérico. Ya que el tiempo de cómputo en la generación de números aleatorios depende, inevitablemente, de los ordenadores empleados, en la Tabla 4.4 se muestran, en tanto por uno respecto al algoritmo propio del lenguaje de programación $\mathrm{C}$ "rand", los tiempos de cómputo empleado por cada generador, junto con su período. Estos resultados permitirán que nos hagamos una idea de la velocidad de generación de cada algoritmo.

\begin{tabular}{|c|c|c|}
\cline { 2 - 3 } \multicolumn{1}{c|}{} & Tiempo de cómputo & Período \\
\hline rand & 1 & $2^{15}$ \\
\hline drand48 & 4,13 & $2^{48}$ \\
\hline ran0 & 2,03 & $2^{31}$ \\
\hline $\operatorname{ran1}$ & 2,25 & $2^{31}$ \\
\hline $\operatorname{ran2}$ & 4,78 & $2^{61}$ \\
\hline ran3 & 0,86 & $2^{30}$ \\
\hline $\operatorname{ran4}$ & 9,53 & $2^{64}$ \\
\hline $\operatorname{ran6}$ & 1,54 & $2^{31}$ \\
\hline
\end{tabular}

Tabla 4.4.-Tiempos de generación en tanto por uno respecto al generador propio del C "rand", y períodos de cada generador.

Como podemos observar en la Tabla 4.4 el generador denominado ran6( ) es el más rápido de entre los que poseen un período de aproximadamente $2^{31}$, prácticamente inagotable para nuestras aplicaciones. Este hecho unido a que fue el que mejor se comportó frente a las pruebas estadísticas, para cortos periodos de generación, y su portabilidad y sencillez, hicieron que nos decidiéramos a adoptarlo como el generador que emplearíamos en esta Tesis.

\subsection{DISEÑO DE EXPERIMENTOS. RESULTADOS}

\subsubsection{Experimento sin considerar la influencia de las condiciones meteorológicas}

\section{Estudio de la evolución temporal de la disponibilidad.}

En primer lugar, nuestro estudio consistió en la obtención, mediante simulación, de un archivo de datos en el que aparezca la disponibilidad acumulada en función del tiempo, considerando como periodo de simulación 200 millones de horas (22.500 años aproximadamente). 


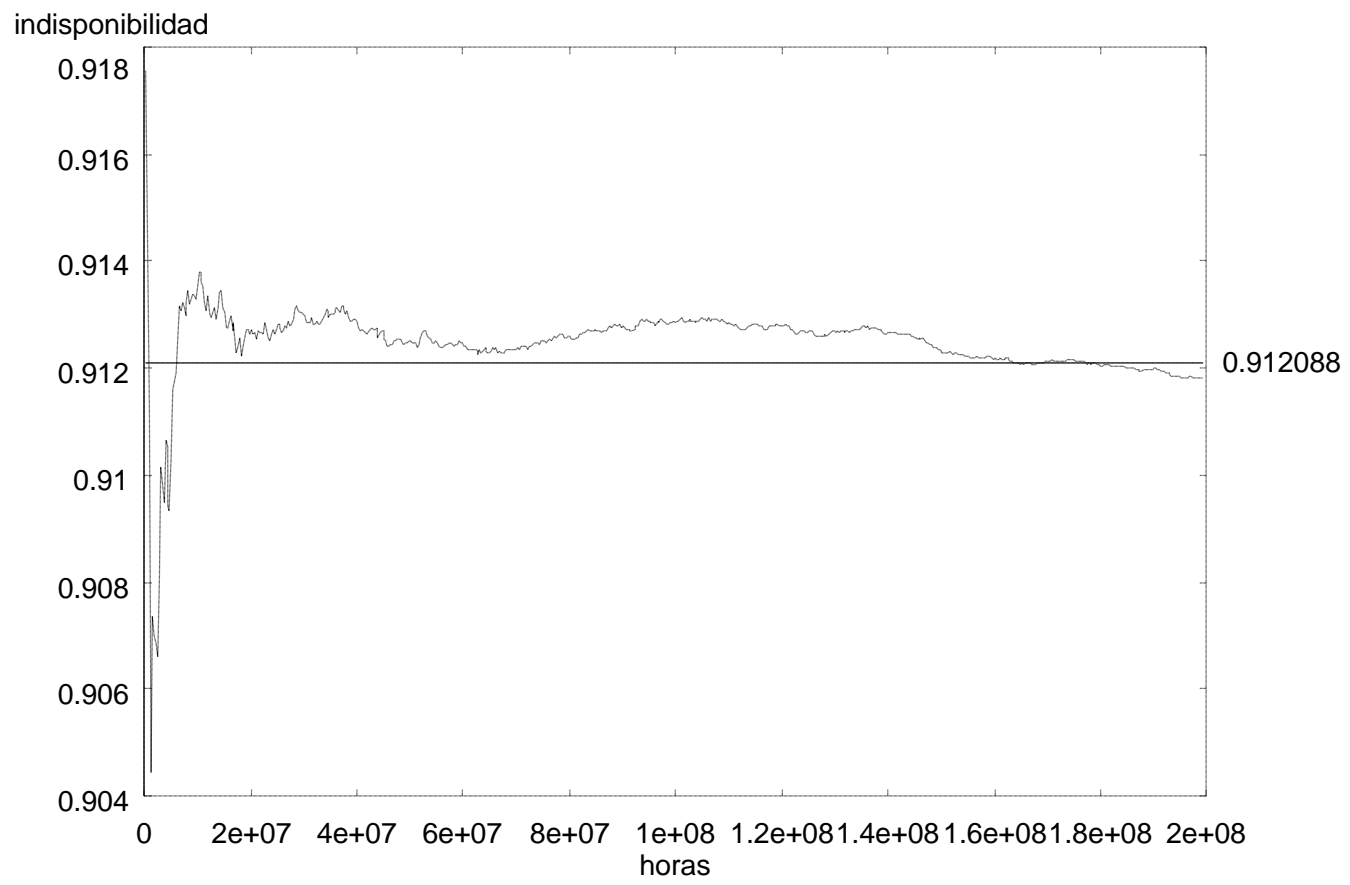

Fig.4.1.-Evolución temporal de la disponibilidad.

En la figura 4.1 se muestra el resultado de la evolución temporal de la disponibilidad, hacia su valor estacionario, obtenido analíticamente aplicando procesos de Markov de espacio de estado discreto y parámetro continuo. Podemos ver que el error se encuentra siempre por debajo del dos por mil a partir de las 10 millones de horas simuladas. El principal problema de este "modus operandi", es la falta de referencia sobre la cota de error de la disponibilidad media que estimamos si no hubiéramos tenido otro método con el que comparar, lo que, a menudo, sucede.

\section{Estudio de la función de distribución de probabilidad de la disponibilidad}

En este segundo caso, el estudio consistió en fijar un período de simulación menor que en el caso anterior, pero repitiendo la simulación un cierto número de veces. Con ello obtendremos varios valores de disponibilidad, uno por simulación, para el período de simulación que hayamos fijado. De estos valores, hallaremos su media y su desviación cuadrática media, que nos darán, respectivamente, la disponibilidad media y una cota del error en el valor de la disponibilidad que nos proporciona cada simulación (L_SOB-76), ya que si empleamos un buen generador de números aleatorios, los valores que obtendremos de la disponibilidad cada vez que realicemos una simulación se distribuirán como una normal alrededor del valor de la disponibilidad media. Por tanto tenemos una forma de estimar de manera bastante precisa el valor de la disponibilidad y su cota de error. 


\begin{tabular}{|c|c|c|c|c|c|c|c|c|}
\cline { 2 - 9 } \multicolumn{1}{c|}{} & \multicolumn{4}{c|}{ Valor medio } & \multicolumn{4}{c|}{ Desviación } \\
\cline { 2 - 9 } \multicolumn{1}{c|}{} & $\mathbf{1 0 0}$ & $\mathbf{5 0 0}$ & $\mathbf{1 . 0 0 0}$ & $\mathbf{1 0 . 0 0 0}$ & $\mathbf{1 0 0}$ & $\mathbf{5 0 0}$ & $\mathbf{1 . 0 0 0}$ & $\mathbf{1 0 . 0 0 0}$ \\
\hline $\mathbf{5 0 . 0 0 0}$ horas &, 915950 &, 912762 &, 912256 &, 911900 &, 020805 &, 020502 &, 020358 &, 020275 \\
\hline $\mathbf{1 0 0 . 0 0 0}$ horas &, 910560 &, 911764 &, 911884 &, 911831 &, 014159 &, 014691 &, 014467 &, 014127 \\
\hline $\mathbf{5 0 0 . 0 0 0}$ horas &, 913000 &, 911900 &, 911863 &, 911804 &, 006629 &, 006146 &, 006336 &, 006377 \\
\hline
\end{tabular}

Tabla 4.5.-Media de la disponibilidad y su desviación cuadrática media según número de experimentos y período de simulación.

En este estudio, los valores para el número de experimentos que empleamos fueron 100, 500, 1.000, y 10.000 experimentos, y los períodos de simulación fueron de 50.000, 100.000, y 500.000 horas. Los resultados obtenidos para estos valores pueden observarse en la Tabla 4.5, donde representamos la media y la desviación cuadrática media de los valores de disponibilidad obtenidos para cada período de simulación y número de experimentos.

En la tabla 4.5 podemos observar que en el peor de los casos (para 50.000 horas y 100 experimentos) el mayor error en la disponibilidad media está por debajo del cinco por mil. En cuanto a la desviación cuadrática media, disminuye según la raíz cuadrada del número de ciclos de funcionamiento-fallo simulados que en nuestro caso es directamente proporcional al período de tiempo simulado (L_SOB-76).

\subsubsection{Experimento considerando la influencia de las condiciones meteorológicas}

En cada uno de los experimentos de simulación descritos en el apartado 2.3, empleamos un tiempo de simulación de 500.000 horas, repitiendo las simulaciones 500 veces, para, más tarde hallar el valor medio y la desviación cuadrática media correspondiente a los 500 valores de indisponibilidad resultado de estas simulaciones. Por tanto tendremos una disponibilidad media y una cota de error para cada modelo de experimento de simulación.

En la Tabla 4.6 se muestran los resultados de disponibilidad media obtenidos mediante simulación, para las dos alternativas de experimento de simulación que hemos considerado, comparados con los resultados obtenidos analíticamente aplicando procesos de Markov de espacio de estado discreto y parámetro continuo, cuando variamos la relación entre la duración media del buen tiempo $N$, y la del mal tiempo $S$, (1000 a 1, 133 a 1, y 2 a 1). Hay que aclarar, que en las casillas con dos valores, uno encima del otro, el valor de la parte superior corresponde a tiempos de reparación o de localización normalmente distribuidos, y el de la parte inferior a tiempo de reparación o de localización exponencialmente distribuidos. 


\begin{tabular}{|c|c|c|c|}
\hline $\begin{array}{c}\mathrm{N}=\text { duración media del buen tiempo } \\
\text { (horas) } \\
\mathrm{S}=\text { duración media del mal tiempo (horas) } \\
\mathbf{N}\end{array}$ & MARKOV & Reparación & $\begin{array}{l}\text { Localización } \\
\text { +Reparación }\end{array}$ \\
\hline 100 & 0,899663 & $\begin{array}{c}\text { Norm: } 0,576080 \\
\text { Exp: } 0,899770\end{array}$ & $\begin{array}{l}0,642714 \\
0,889002\end{array}$ \\
\hline 1000 & 0,899947 & $\begin{array}{c}\text { Norm: } 0,882712 \\
\text { Exp: } 0,901040\end{array}$ & $\begin{array}{l}0,888020 \\
0,901198\end{array}$ \\
\hline 10000 & 0,904708 & $\begin{array}{c}\text { Norm: } 0,905508 \\
\text { Exp: } 0,907124\end{array}$ & $\begin{array}{l}0,905996 \\
0,907242\end{array}$ \\
\hline $100000 \quad 100$ & 0,910061 & $\begin{array}{c}\text { Norm: } 0,911236 \\
\text { Exp: } 0,910328\end{array}$ & $\begin{array}{l}0,910938 \\
0,911336\end{array}$ \\
\hline 0,3 & 0,821277 & $\begin{array}{c}\text { Norm: } 0,167490 \\
\text { Exp: } 0,823280\end{array}$ & $\begin{array}{l}0,181736 \\
0,764698\end{array}$ \\
\hline 200 & 0,827708 & $\begin{array}{c}\text { Norm: } 0,671564 \\
\text { Exp: } 0,836222\end{array}$ & $\begin{array}{l}0,711192 \\
0,832086\end{array}$ \\
\hline 2000 & 0,868626 & $\begin{array}{c}\text { Norm: } 0,871886 \\
\text { Exp: } 0,879528\end{array}$ & $\begin{array}{l}0,874720 \\
0,881742 \\
\end{array}$ \\
\hline 20000 & 0,899645 & $\begin{array}{c}\text { Norm: } 0,904634 \\
\text { Exp: } 0,904960 \\
\end{array}$ & $\begin{array}{l}0,904688 \\
0,905548\end{array}$ \\
\hline 2000001500 & 0,904701 & $\begin{array}{c}\text { Norm: } 0,909108 \\
\text { Exp: } 0,908466\end{array}$ & $\begin{array}{l}0,908872 \\
0,908898\end{array}$ \\
\hline 200 & 0,389496 & $\begin{array}{c}\text { Norm: } 0,296830 \\
\text { Exp: } 0,444696\end{array}$ & $\begin{array}{l}0,326666 \\
0,440242\end{array}$ \\
\hline 2000 & 0,571011 & $\begin{array}{c}\text { Norm: } 0,665872 \\
\text { Exp: } 0,663524\end{array}$ & $\begin{array}{l}0,669582 \\
0,676580\end{array}$ \\
\hline
\end{tabular}

Tabla 4.6.-Resultados para una relación N/S de 1000 a 1, de 133 a 1 y de 2 a 1 , comparados con los alcanzados mediante procesos de Markov.

Del examen de los resultados reflejados en la Tabla 4.6, se deduce que, a medida que disminuye el número medio de horas de buen tiempo y el de mal tiempo, los resultados mediante simulación difieren más de los obtenidos por Markov. Esto se acentúa especialmente en el caso de tiempos de fallo distribuidos normalmente. La explicación de este hecho es que, al disminuir los ciclos de buen y mal tiempo, estos resultan cada vez de menor duración comparados con los de funcionamiento del elemento, por lo que al pasar de un intervalo de buen tiempo a otro de mal tiempo o viceversa "interrumpimos" el normal funcionamiento de los elementos un número mayor de veces puesto que debemos cambiar los valores de sus tasas de fallo para cada condición meteorológica. Si, además, resulta que el tiempo medio de reposición es mayor que la duración media de los ciclos de buen tiempo, cuando un elemento entre en fallo será difícil que salga de él y por tanto dará lugar a valores de la disponibilidad anormalmente bajos respecto a los valores calculados por Markov. Además, debido a estos "cortes", muy frecuentes, el proceso pierde el carácter markoviano, y por consiguiente se produce diferencia entre los resultados para distribución normal y para distribución exponencial de los tiempos de reparación y localización. 
También podemos observar que, cuando la casi totalidad del período de simulación corresponde a intervalos de buen tiempo (lo que corresponde a una duración media del buen tiempo muy elevada respecto a la del mal tiempo), el tipo de distribución del tiempo hasta el fallo (exponencial o normal) y la separación o no del intervalo de tiempo que dura la reposición de servicio, en localización y reparación, apenas influyen en el resultado de la simulación, obteniendo, en todos los casos, resultados bastante parecidos a los de Markov. Esto es debido a que, a diferencia del caso anterior, apenas se pierde el carácter markoviano del experimento al ser más pequeña la duración media del tiempo de funcionamiento de los elementos que la duración media de los ciclos de buen tiempo.

\section{2 ÍNDICES DE CALIDAD. RESULTADOS}

Intentaremos analizar la influencia que, en los índices de calidad (definidos en el apartado 1.0.4), de las tres redes de ensayo, ver apartado 3.1, diseñadas en esta Tesis, tienen las siguientes características de la distribución de los tiempos entre eventos:

- Influencia del tipo de distribución elegida para el tiempo de reparación de los elementos que componen la red. Modelización realista de algunos sucesos.

- Influencia de la duración media del tiempo de localización y desplazamiento asociado a las interrupciones de suministro. Acciones de explotación.

- Influencia de las duraciones medias de los ciclos de buen y mal tiempo, $N$ y $S$ respectivamente.

- Influencia relativa de los distintos elementos que componen cada red en sus índices de calidad. Esta influencia la estudiaremos a partir de las tasa de fallo y tiempos de reparación de los elementos. Permite examinar acciones de Planificación y Mantenimiento.

Utilizaremos, por tanto, los cuatro escenarios diseñados en el apartado 3.1.1 para poder determinar cada una de las influencias, antes descritas, sobre los índices de calidad. Las unidades en las que se darán todos los resultados, pertenecientes a los cuatro escenarios diseñados, son los correspondientes a la definición de cada índice de calidad que hicimos en el apartado 1.0.4.

\section{Escenario CAL-1}

En este escenario evaluaremos la influencia del tipo de distribución elegida para el tiempo de reparación de los elementos que componen las redes que se han diseñado, comparando, también, los resultados producto de nuestra simulación con los obtenidos mediante procesos de Markov. 
Los cálculos se llevaron a cabo para las tres redes diseñadas bajo las condiciones descritas en el apartado 3.1.1.

En la primera columna de las Tablas 4.7, 4.8 y 4.9 se pueden apreciar los resultados obtenidos utilizando como parámetros de fiabilidad de los componentes los del Anexo 2. En la segunda columna utilizaremos esos mismos parámetros excepto para la línea aérea LA1 y el cable subterráneo CS0, cuyas tasas de fallo y tiempos de reparación se dividirán, conjuntamente, por 4 . En la tercera columna se multiplicarán por 10 .

\begin{tabular}{|c|c|c|c|c|c|c|c|c|c|}
\hline & \multicolumn{9}{|c|}{ Tasas de Fallo y Tiempos de Reparación de los elementos de la red } \\
\hline & \multicolumn{3}{|c|}{ Caso Base } & \multicolumn{3}{|c|}{$\begin{array}{c}\text { Caso Base excepto en } \\
\text { LA1 x } 0,25\end{array}$} & \multicolumn{3}{|c|}{$\begin{array}{c}\text { Caso Base excepto en } \\
\text { LA } 1 \text { x } 10\end{array}$} \\
\hline & Markov & Expo. & Normal & Markov & Expo. & Normal & Markov & Expo. & Normal \\
\hline TIEPI & 8.104955 & 8,111493 & 8,044008 & 2,362733 & 2,420121 & 2,401445 & 203,2327 & 189,8503 & 188,2210 \\
\hline \multicolumn{2}{|c|}{$\%$ respecto Markov } & $0,08 \%$ & $-0,75 \%$ & & $2,43 \%$ & $1,63 \%$ & & $-6,58$ & $-7,38 \%$ \\
\hline TIEB & 7,963299 & 7,965488 & 7,903908 & 2,321403 & 2,363591 & 2,355313 & 200,7752 & 187,8122 & 186,0797 \\
\hline \multicolumn{2}{|c|}{$\%$ respecto Markov } & $0,03 \%$ & $-0,74 \%$ & & $1,81 \%$ & $1,46 \%$ & & $-6,45 \%$ & $-7,31 \%$ \\
\hline NIEPI & 1,594360 & 1,582885 & 1,576852 & 0,658856 & 0,668909 & 0,667736 & 12,69910 & 11,17025 & 11,14862 \\
\hline \multicolumn{2}{|c|}{$\%$ respecto Markov } & $-0,71 \%$ & $-1,09 \%$ & & $1,52 \%$ & $1,35 \%$ & & $-12,03 \%$ & $-12,21 \%$ \\
\hline NIEB & 1,560843 & 1,548378 & 1,543901 & 0,645886 & 0,654810 & 0,653253 & 12,42206 & 10,95859 & 10,92608 \\
\hline \multicolumn{2}{|c|}{$\%$ respecto Markov } & $-0,80 \%$ & $-1,08 \%$ & & $1,38 \%$ & $1,14 \%$ & & $-11,78 \%$ & $-12,04 \%$ \\
\hline IIS & 0,925223 & 0,925970 & 0,918266 & 0,269718 & 0,276269 & 0,274138 & 23,20006 & 21,67241 & 21,48641 \\
\hline \multicolumn{2}{|c|}{$\%$ respecto Markov } & $0,08 \%$ & $-0,75 \%$ & & $2,43 \%$ & $1,64 \%$ & & $-6,58 \%$ & $-7,38 \%$ \\
\hline ENS & 10,89305 & 10,90184 & 10,81114 & 3,175514 & 3,252642 & 3,227543 & 273,1447 & 255,1588 & 252,9690 \\
\hline \multicolumn{2}{|c|}{$\%$ respecto Markov } & $0,08 \%$ & $-0,75 \%$ & & $2,43 \%$ & $1,64 \%$ & & $-6,74 \%$ & $-7,38 \%$ \\
\hline $\mathrm{K}$ & 28,09516 & 27,87081 & 27,79022 & 11,62595 & 11,78657 & 11,75854 & 223,5971 & 197,2546 & 196,6694 \\
\hline \multicolumn{2}{|c|}{$\%$ respecto Markov } & $-0,8 \%$ & $-1,08 \%$ & & $1,38 \%$ & $1,14 \%$ & & $-11,78 \%$ & $-12,04 \%$ \\
\hline
\end{tabular}

Tabla 4.7.-Influencia del tipo de distribución empleada para el tiempo de reparación de los elementos de la red aérea. Resultados para distintas características de fiabilidad.

Para la red subterránea, véase Tabla 4.8, los índices de calidad obtenidos mediante simulación son similares a los obtenidos mediante procesos de Markov para los tres casos analizados, no llegando nunca a alcanzarse una disparidad del $1,25 \%$. Sin embargo, para las redes con elementos sujetos a las condiciones meteorológicas, como son la red aérea y la red mixta (véanse Tablas 4.7 y 4.9), existen una diferencias, para el supuesto en que multiplicamos por 10 las tasas de fallo y tiempos de reparación de los elementos CS0 y LA1, que pueden llegar a un $12 \%$ en los índices relacionados con el número de interrupciones y a la mitad de este porcentaje en los índices relacionados con la duración de las interrupciones.

Esta diferencia de resultados es debida al solapamiento que se produce en las interrupciones, pues se concentran la mayoría durante el mal tiempo, no contabilizandose separadamente todos los fallos de los elementos que provocan indisponibilidad, ni su duración. Este efecto, no es tenido en cuenta en la técnica 
basada en procesos de Markov, que supone que los fallos de los elementos que producen indisponibilidad no se solapan.

\begin{tabular}{|c|c|c|c|c|c|c|c|c|c|}
\hline & \multicolumn{9}{|c|}{ Tasas de Fallo y Tiempos de Reparación de los elementos de la red } \\
\hline & \multicolumn{3}{|c|}{ Caso Base } & \multicolumn{3}{|c|}{$\begin{array}{c}\text { Caso Base excepto en } \\
\text { LA1 x } 0,25\end{array}$} & \multicolumn{3}{|c|}{$\begin{array}{c}\text { Caso Base excepto en } \\
\text { LA } 1 \text { x } 10\end{array}$} \\
\hline & Markov & Expo. & Normal & Markov & Expo. & Normal & Markov & Expo. & Normal \\
\hline TIEPI & 2,546971 & 2,536917 & 2,548042 & 0,986299 & 0,987815 & 0,990369 & 84,50086 & 84,36398 & 84,27898 \\
\hline \multicolumn{2}{|c|}{$\%$ respecto Markov } & $-0,39 \%$ & $-0,04 \%$ & & $0,15 \%$ & $0,41 \%$ & & $-0,16 \%$ & $-0,26 \%$ \\
\hline TIEB & 2,557998 & 2,552843 & 2,559777 & 0,990501 & 0,992146 & 0,993744 & 84,91466 & 84,76818 & 84,66003 \\
\hline \multicolumn{2}{|c|}{$\%$ respecto Markov } & $-0,20 \%$ & $0,07 \%$ & & $0,16 \%$ & $0,33 \%$ & & $-0,17 \%$ & $-0,30 \%$ \\
\hline NIEPI & 0,385471 & 0,385004 & 0,384962 & 0,305030 & 0,304772 & 0,305722 & 1,350756 & 1,335281 & 1,336148 \\
\hline \multicolumn{2}{|c|}{$\%$ respecto Markov } & $-0,12 \%$ & $-0,13 \%$ & & $-0,08 \%$ & $0,23 \%$ & & $-1,14 \%$ & $-1,08 \%$ \\
\hline NIEB & 0,385724 & 0,385310 & 0,385821 & 0,305603 & 0,305375 & 0,306458 & 1,347172 & 1,331730 & 1,332289 \\
\hline \multicolumn{2}{|c|}{$\%$ respecto Markov } & $-0,11 \%$ & $-0,02 \%$ & & $-0,07 \%$ & $0,28 \%$ & & $-1,14 \%$ & $-1,10 \%$ \\
\hline \begin{tabular}{l|} 
IIS \\
\end{tabular} & 0,290750 & 0,289602 & 0,290872 & 0,112591 & 0,112764 & 0,113056 & 9,646217 & 9,630591 & 9,620889 \\
\hline \multicolumn{2}{|c|}{$\%$ respecto Markov } & $-0,39 \%$ & $-0,04 \%$ & & $0,15 \%$ & $0,41 \%$ & & $-0,16 \%$ & $-0,26 \%$ \\
\hline \begin{tabular}{l|} 
ENS \\
\end{tabular} & 21,03201 & 21,10968 & 21,20226 & 8,143539 & 8,219606 & 8,240858 & 697,6956 & 701,9927 & 701,2855 \\
\hline \multicolumn{2}{|c|}{$\%$ respecto Markov } & $0,37 \%$ & $0,81 \%$ & & $0,93 \%$ & $1,19 \%$ & & $0,61 \%$ & $0,51 \%$ \\
\hline $\mathrm{K}$ & 11,18600 & 11,17399 & 11,18879 & 8,862500 & 8,855878 & 8,887282 & 39,06800 & 38,62016 & 38,63637 \\
\hline \multicolumn{2}{|c|}{$\%$ respecto Markov } & $-0,11 \%$ & $0,02 \%$ & & $-0,07 \%$ & $0,28 \%$ & & $-1,14 \%$ & $-1,10 \%$ \\
\hline
\end{tabular}

Tabla 4.8.-Influencia del tipo de distribución empleada para el tiempo de reparación de los elementos de la red subterránea. Resultados para distintas características de fiabilidad.

\begin{tabular}{|c|c|c|c|c|c|c|c|c|c|}
\hline & \multicolumn{9}{|c|}{ Tasas de Fallo y Tiempos de Reparación de los elementos de la red } \\
\hline & \multicolumn{3}{|c|}{ Caso Base } & \multicolumn{3}{|c|}{$\begin{array}{c}\text { Caso Base excepto en } \\
\text { LA1 x } 0,25\end{array}$} & \multicolumn{3}{|c|}{$\begin{array}{c}\text { Caso Base excepto en } \\
\text { LA } 1 \text { × } 10\end{array}$} \\
\hline & Markov & Expo. & Normal & Markov & Expo. & Normal & Markov & Expo. & Normal \\
\hline TIEPI & 7,361616 & 7,359767 & 7,358951 & 2,096938 & 2,111887 & 2,114762 & 217,8756 & 208,1180 & 209,3765 \\
\hline \multicolumn{2}{|c|}{$\%$ respecto Markov } & $-0,02 \%$ & $-0,03 \%$ & & $0,71 \%$ & $0,85 \%$ & & $-4,48 \%$ & $-3,90 \%$ \\
\hline TIEB & 7,282471 & 7,282107 & 7,271161 & 2,097143 & 2,110746 & 2,118641 & 207,9590 & 197,8397 & 199,2543 \\
\hline \multicolumn{2}{|c|}{$\%$ respecto Markov } & $-0,005 \%$ & $-0,15 \%$ & & $0,65 \%$ & $1,02 \%$ & & $-4,86 \%$ & $-4,18 \%$ \\
\hline NIEPI & 1,335664 & 1,328792 & 1,325185 & 0,633135 & 0,635654 & 0,635629 & 9,682476 & 8,803814 & 8,843657 \\
\hline \multicolumn{2}{|c|}{$\%$ respecto Markov } & $-0,51 \%$ & $-0,78 \%$ & & $0,40 \%$ & $0,39 \%$ & & $-9,07 \%$ & $-8,66 \%$ \\
\hline NIEB & 1,346909 & 1,342351 & 1,336271 & 0,621750 & 0,624789 & 0,624417 & 9,960833 & 9,013737 & 9,060959 \\
\hline \multicolumn{2}{|c|}{$\%$ respecto Markov } & $-0,33 \%$ & $-0,79 \%$ & & $0,49 \%$ & $0,43 \%$ & & $-9,50 \%$ & $-9,03 \%$ \\
\hline IIS & 0,840367 & 0,840156 & 0,840063 & 0,239377 & 0,241083 & 0,241411 & 24,87165 & 23,75777 & 23,90143 \\
\hline \multicolumn{2}{|c|}{$\%$ respecto Markov } & $-0,02 \%$ & $-0,03 \%$ & & $0,71 \%$ & $0,85 \%$ & & $-4,48 \%$ & $-3,90 \%$ \\
\hline ENS & 41,37666 & 41,42812 & 41,42351 & 11,79551 & 11,88780 & 11,90399 & 1218,420 & 1171,496 & 1178,580 \\
\hline \multicolumn{2}{|c|}{$\%$ respecto Markov } & $0,12 \%$ & $0,11 \%$ & & $0,78 \%$ & $0,92 \%$ & & $-3,85 \%$ & $-3,27 \%$ \\
\hline $\mathrm{K}$ & 79,46762 & 79,19871 & 78,84001 & 36,68058 & 36,86251 & 36,84061 & 587,6895 & 531,8104 & 534,5965 \\
\hline \multicolumn{2}{|c|}{$\%$ respecto Markov } & $-0,34 \%$ & $-0,45 \%$ & & $0,49 \%$ & $0,43 \%$ & & $-9,51 \%$ & $-9,03 \%$ \\
\hline
\end{tabular}

Tabla 4.9.-Influencia del tipo de distribución empleada para el tiempo de reparación de los elementos de la red mixta. Resultados para distintas características de fiabilidad. 
Podemos concluir, sin embargo, que excepto en los escenarios en los que sea anormalmente elevada la tasa de fallo de algún elemento o la duración media de los ciclos de mal tiempo, $S$, las hipótesis simplificadoras que, normalmente, se hacen en la técnica basada en los procesos de Markov, para la obtención de los índices de calidad de la red, son bastante razonables.

También podemos concluir, a la vista de las tablas 4.7, 4.8 y 4.9, la escasa influencia, que sobre los índices de calidad, tiene la utilización de una distribución exponencial o normal truncada para los tiempos de reparación de los elementos que forman las redes de ensayo.

\section{Escenario CAL-2}

En este escenario evaluaremos la influencia de la duración media del tiempo de localización y desplazamiento asociado a las interrupciones de suministro en los índices de calidad de las redes de ensayo.

Se utilizan, como características de fiabilidad de los elementos de la red, los valores de las tasas de fallo y tiempos de reparación que aparecen en el Anexo 2. Las condiciones que impondremos a nuestra simulación son las expuestas en el apartado 3.1.1.

Utilizaremos ocho valores distintos para la duración media del tiempo de desplazamiento y localización con el fin de obtener las variaciones que esta variable produce en los índices de calidad de la red.

En la Tabla 4.10, 4.11 y 4.12 se pueden observar los resultados obtenidos para las tres redes de ensayo utilizadas.

\begin{tabular}{|c|c|c|c|c|c|c|c|c|}
\hline Índices & \multicolumn{7}{|c|}{ Tiempo de Localización y Desplazamiento (horas) } \\
\hline & 0 & 0,5 & 1 & 1,5 & 2 & 3 & 4 & 5 \\
\hline TIEPI & 5,646686 & 6,488254 & 7,345310 & 8,111493 & 8,816841 & 10,58463 & 12,10564 & 13,66401 \\
\hline TIEB & 5,572487 & 6,367311 & 7,208455 & 7,965488 & 8,649644 & 10,40742 & 11,87503 & 13,38197 \\
\hline NIEPI & 1,570251 & 1,582961 & 1,594244 & 1,582885 & 1,568108 & 1,593063 & 1,579365 & 1,580945 \\
\hline NIEB & 1,541346 & 1,548671 & 1,557820 & 1,548378 & 1,536090 & 1,564098 & 1,545965 & 1,544169 \\
\hline IIS & 0,644599 & 0,740668 & 0,838506 & 0,925970 & 1,006489 & 1,208294 & 1,381923 & 1,559819 \\
\hline ENS & 7,589146 & 8,720213 & 9,872096 & 10,90184 & 11,84983 & 14,22574 & 16,26999 & 18,36444 \\
\hline K & 27,74423 & 27,87607 & 28,04076 & 27,87081 & 27,64962 & 28,15376 & 27,82701 & 27,79503 \\
\hline
\end{tabular}

Tabla 4.10.-Variación de los Índices de Calidad en función del tiempo de localización y desplazamiento para la red aérea en caso de avería.

\begin{tabular}{|l|l|}
\hline Índices & Tiempo de Localización y Desplazamiento (horas) \\
\hline
\end{tabular}




\begin{tabular}{|c|c|c|c|c|c|c|c|c|}
\hline & 0 & 0,5 & 1 & 1,5 & 2 & 3 & 4 & 5 \\
\hline TIEPI & 1,958810 & 2,172837 & 2,374296 & 2,536917 & 2,735570 & 3,130869 & 3,496669 & 3,914447 \\
\hline TIEB & 1,967808 & 2,181311 & 2,384091 & 2,552843 & 2,756694 & 3,145131 & 3,504225 & 3,919231 \\
\hline NIEPI & 0,386134 & 0,385596 & 0,386821 & 0,385004 & 0,384964 & 0,384951 & 0,384706 & 0,386326 \\
\hline NIEB & 0,385855 & 0,385919 & 0,386642 & 0,385310 & 0,385523 & 0,385473 & 0,384647 & 0,386440 \\
\hline IIS & 0,223608 & 0,248041 & 0,271038 & 0,289602 & 0,312280 & 0,357405 & 0,399163 & 0,446855 \\
\hline ENS & 16,29926 & 18,08017 & 19,75652 & 21,10968 & 22,76267 & 26,05196 & 29,09578 & 32,57211 \\
\hline K & 11,18980 & 11,19164 & 11,21262 & 11,17399 & 11,18017 & 11,17872 & 11,15476 & 11,20675 \\
\hline
\end{tabular}

Tabla 4.11.-Variación de los Índices de Calidad en función del tiempo de localización y desplazamiento para la red subterránea en caso de avería.

\begin{tabular}{|c|c|c|c|c|c|c|c|c|}
\hline Índices & \multicolumn{7}{|c|}{ Tiempo de Localización y Desplazamiento (horas) } \\
\hline & 0 & 0,5 & 1 & 1,5 & 2 & 3 & 4 & 5 \\
\hline TIEPI & 5,363163 & 6,031231 & 6,678295 & 7,359767 & 8,016514 & 9,377339 & 10,72119 & 12,07329 \\
\hline TIEB & 5,264341 & 5,945030 & 6,577283 & 7,282107 & 7,952377 & 9,308510 & 10,66648 & 12,05850 \\
\hline NIEPI & 1,330585 & 1,327808 & 1,326134 & 1,328792 & 1,326431 & 1,329630 & 1,331861 & 1,324545 \\
\hline NIEB & 1,340577 & 1,338929 & 1,335239 & 1,342351 & 1,338550 & 1,340094 & 1,341950 & 1,336405 \\
\hline IIS & 0,612233 & 0,688497 & 0,762362 & 0,840156 & 0,915127 & 1,070472 & 1,223881 & 1,378230 \\
\hline ENS & 30,18923 & 33,94978 & 37,59211 & 41,42812 & 45,12494 & 52,78502 & 60,34957 & 67,96056 \\
\hline K & 79,09402 & 78,99681 & 78,77911 & 79,19871 & 78,97445 & 79,06557 & 79,17507 & 78,84788 \\
\hline
\end{tabular}

Tabla 4.12.-Variación de los Índices de Calidad en función del tiempo de localización y desplazamiento para la red mixta en caso de avería.

A continuación, para una mejor apreciación de estos resultados, se ofrecen en las figuras 4.2, 4.3 y 4.4 la representación de los índices TIEPI, TIEB, IIS y ENS. El resto de los índices no se representarán puesto que conciernen al número de interrupciones y, como cabría esperar, no experimentan cambios al variar el tiempo de localización y desplazamiento, como se puede observar en las Tablas 4.10, 4.11 y 4.12 .

Como se ve en las figuras 4.2, 4.3 y 4.4 la dependencia del TIEPI, TIEB, IIS y ENS, con el tiempo de desplazamiento es prácticamente lineal.

Se puede concluir que disminuyendo el tiempo medio de localización y desplazamiento incrementamos linealmente, en la proporción que se ve en las figuras 4.2, 4.3 y 4.4, los índices de calidad de una red. La forma de disminuir este tiempo medio de localización y desplazamiento, puede ser a través del aumento del número de retenes de operarios que se encargan de la reposición de servicio, la modificación de sus emplazamientos o la mejora en los medios técnicos utilizados por estos, entre otras posibilidades. 


\section{CAPÍTULO 4 - RESULTADOS}

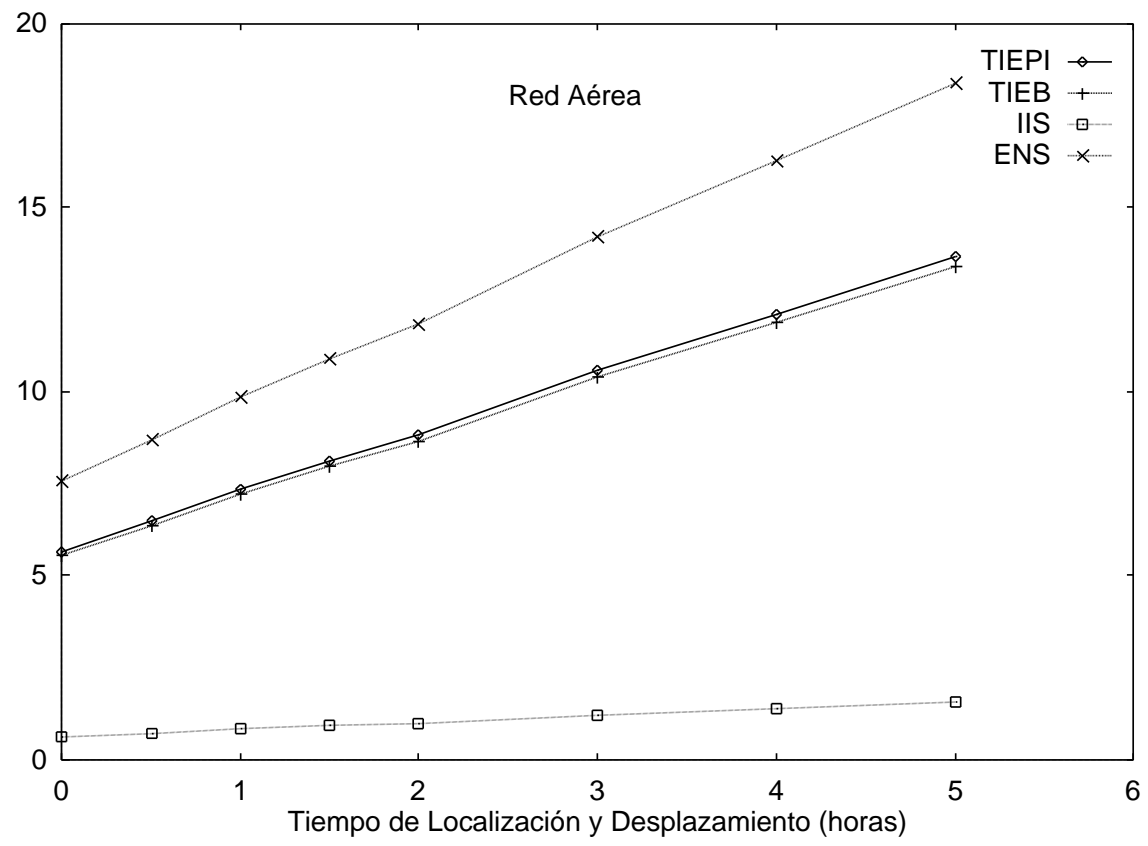

Fig.4.2.-Variación de los Índices de Calidad en función del tiempo de localización y desplazamiento para la red aérea en caso de avería.

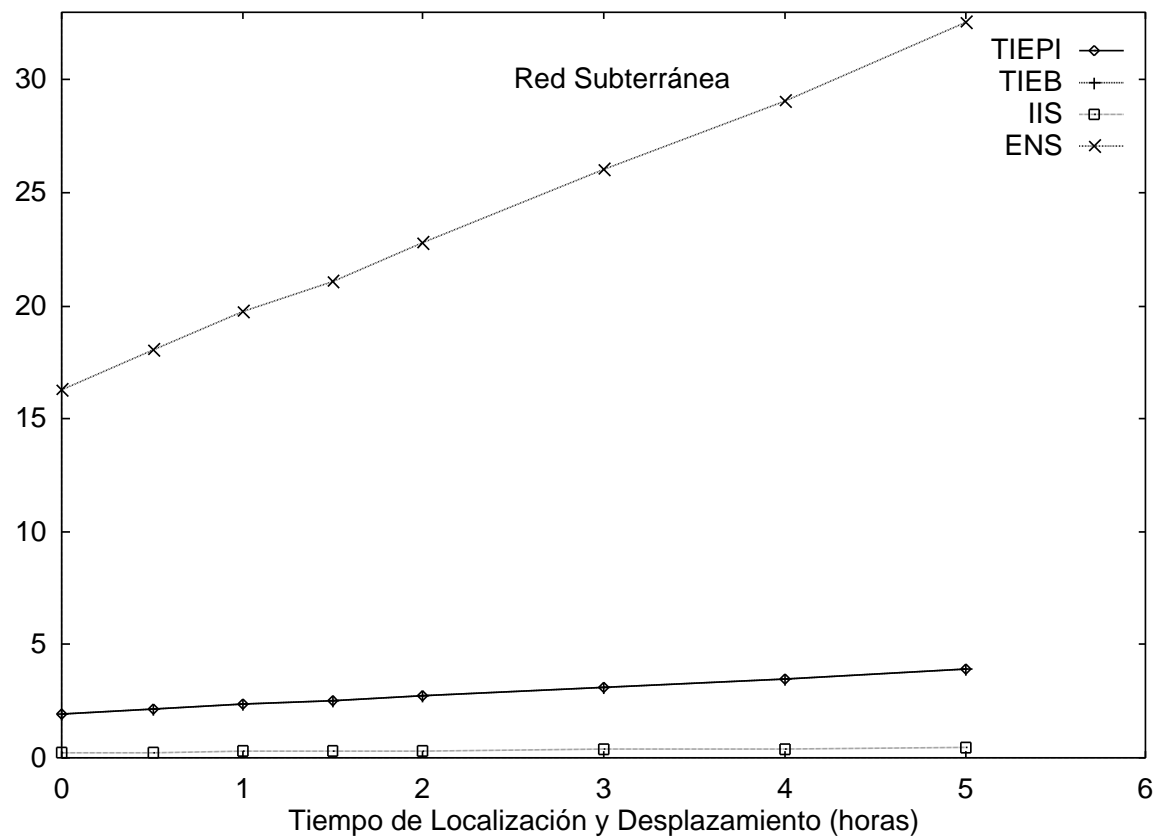

Fig.4.3.-Variación de los Índices de Calidad en función del tiempo de localización y desplazamiento para la red subterránea en caso de avería. 


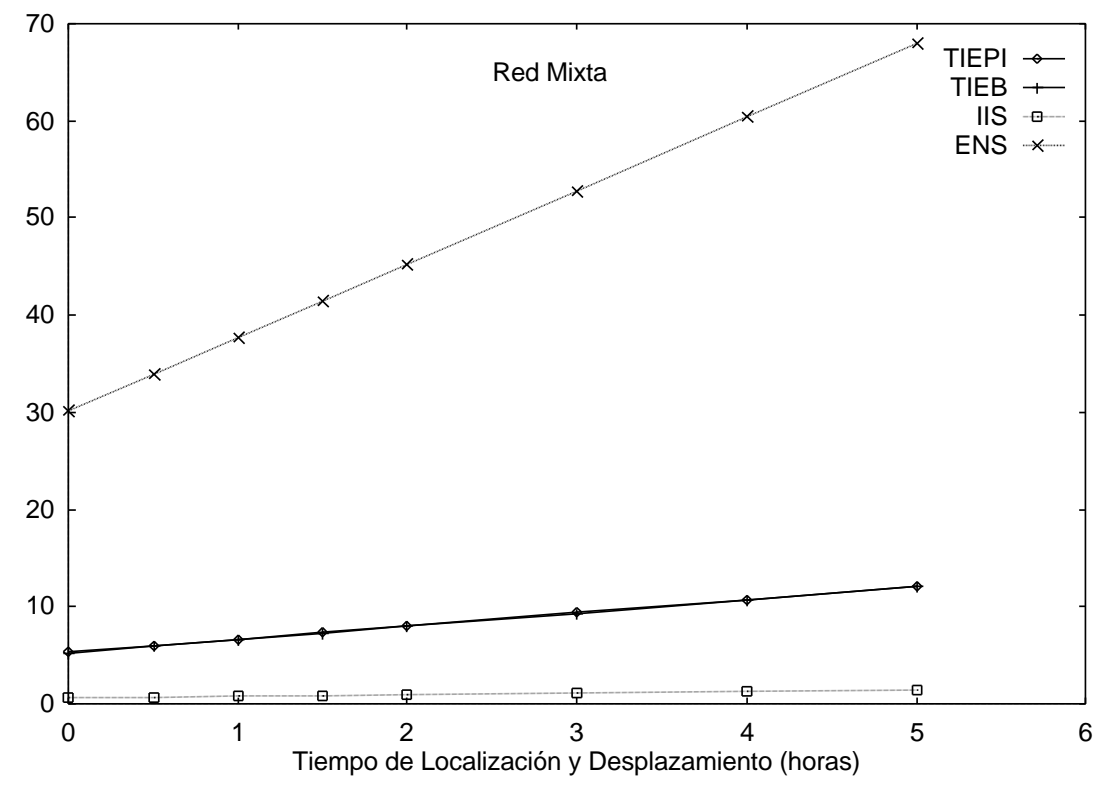

Fig.4.4.-Variación de los Índices de Calidad en función del tiempo de localización y desplazamiento para la red mixta en caso de avería.

\section{Escenario CAL-3}

En este escenario evaluaremos la influencia de las duraciones medias de los ciclos de buen y mal tiempo, $N$ y $S$ respectivamente. Como en el anterior escenario, se utilizarán, como características de fiabilidad de los elementos de la red, los valores de las tasas de fallo y tiempos de reparación que aparecen en el Anexo 2. Las condiciones que impondremos a nuestra simulación son las expuestas en el apartado 3.1.1.

Utilizaremos siete combinaciones distintas para las duraciones medias de los ciclos de buen y mal tiempo con el fin de obtener las variaciones que esta variable produce en los índices de calidad de la red. En las Tablas 4.13 y 4.14 se recogen los resultados obtenidos para las dos redes de ensayo con elementos sujetos a las condiciones meteorológicas.

Como se ve en estas Tablas, el comportamiento de ambas redes es muy similar frente a los cambios en las condiciones meteorológicas, por tanto mencionaremos sus efectos, en ambas, como si de una sola red se tratase.

Si aumentamos la duración de los intervalos de mal tiempo frente a los de bueno, o disminuimos la duración de los intervalos de buen tiempo frente a los de malo, el efecto es similar, aumenta el número de interrupciones hasta el límite impuesto por su duración, esto es, como ocurre en el caso en que $\mathrm{N}=400 \mathrm{~h}$ y $\mathrm{S}=800$ $\mathrm{h}$, no dejamos que los elementos se averíen repetidamente pues, una vez que se han averiado, permanecen en ese estado la mayor parte del tiempo ya que las interrupciones no pueden reponerse hasta que no finaliza el mal tiempo. 
Debido a que las interrupciones no pueden reponerse hasta finalizado el mal tiempo, es mucho más espectacular el crecimiento de los índices relacionados con la duración de las interrupciones (TIEPI, TIEB, IIS y ENS) cuando aumentamos la duración de los intervalos de mal tiempo frente a los de bueno que cuando disminuimos la duración de los de buen tiempo frente a los de malo.

\begin{tabular}{|c|c|c|c|c|c|c|c|c|}
\hline Índices & \multicolumn{7}{|c|}{ Duraciones medias de los ciclos de buen y mal tiempo (horas) } \\
\hline & $\begin{array}{c}\mathrm{N}=400 \\
\mathrm{~S}=0\end{array}$ & $\begin{array}{c}\mathrm{N}=400 \\
\mathrm{~S}=20\end{array}$ & $\begin{array}{c}\mathrm{N}=400 \\
\mathrm{~S}=200\end{array}$ & $\begin{array}{c}\mathrm{N}=400 \\
\mathrm{~S}=800\end{array}$ & $\begin{array}{c}\mathrm{N}=500 \\
\mathrm{~S}=2\end{array}$ & $\begin{array}{c}\mathrm{N}=200 \\
\mathrm{~S}=2\end{array}$ & $\begin{array}{c}\mathrm{N}=100 \\
\mathrm{~S}=2\end{array}$ & $\begin{array}{c}\mathrm{N}=10 \\
\mathrm{~S}=2\end{array}$ \\
\hline TIEPI & 3,951500 & 119,4346 & 2250,56 & 5431,542 & 7,273372 & 12,38737 & 20,26512 & 159,0149 \\
\hline TIEB & 3,898538 & 117,4625 & 2234,129 & 5418,842 & 7,139452 & 12,10736 & 19,91410 & 156,1041 \\
\hline NIEPI & 0,982197 & 5,467370 & 11,23842 & 6,914645 & 1,461718 & 2,197102 & 3,338853 & 20,85182 \\
\hline NIEB & 0,961532 & 5,371633 & 11,15449 & 6,893511 & 1,431846 & 2,146492 & 3,271081 & 20,40744 \\
\hline IIS & 0,451084 & 13,63409 & 256,9140 & 620,0391 & 0,830294 & 1,414083 & 2,313371 & 18,15239 \\
\hline ENS & 5,310815 & 160,5201 & 3024,762 & 7299,993 & 9,775412 & 16,64862 & 27,23633 & 213,7160 \\
\hline K & 17,30757 & 96,68937 & 200,7809 & 124,0831 & 25,77323 & 38,63686 & 58,87946 & 367,3339 \\
\hline
\end{tabular}

Tabla 4.13.-Variación de los Índices de Calidad en función de la duración media de los ciclos de buen y mal tiempo, $N$ y $S$, para la red aérea.

\begin{tabular}{|c|c|c|c|c|c|c|c|c|}
\hline Índices & \multicolumn{7}{|c|}{ Duraciones medias de los ciclos de buen y mal tiempo (horas) } \\
\hline & $\mathrm{N}=400$ & $\mathrm{~N}=400$ & $\mathrm{~N}=400$ & $\mathrm{~N}=400$ & $\mathrm{~N}=500$ & $\mathrm{~N}=200$ & $\mathrm{~N}=100$ & $\mathrm{~N}=10$ \\
$\mathrm{~S}=0$ & $\mathrm{~S}=20$ & $\mathrm{~S}=200$ & $\mathrm{~S}=800$ & $\mathrm{~S}=2$ & $\mathrm{~S}=2$ & $\mathrm{~S}=2$ & $\mathrm{~S}=2$ \\
\hline TIEPI & 4,244182 & 93,83100 & 2014,166 & 5282,836 & 6,730459 & 10,39714 & 16,67651 & 121,4050 \\
\hline TIEB & 4,059703 & 97,03613 & 2044,141 & 5308,685 & 6,645307 & 10,41719 & 16,92142 & 125,0835 \\
\hline NIEPI & 0,901463 & 4,319837 & 10,08785 & 6,682265 & 1,242822 & 1,746038 & 2,590415 & 15,02405 \\
\hline NIEB & 0,892228 & 4,449777 & 10,21886 & 6,704622 & 1,250483 & 1,777493 & 2,665119 & 15,71214 \\
\hline IIS & 0,484496 & 10,71130 & 229,9277 & 603,0635 & 0,768317 & 1,186889 & 1,903712 & 13,85902 \\
\hline ENS & 23,89049 & 528,1745 & 11337,74 & 29737,07 & 37,88574 & 58,52551 & 93,87207 & 683,3886 \\
\hline K & 52,64145 & 262,5368 & 602,9127 & 395,5727 & 73,77846 & 104,8721 & 157,2420 & 927,0164 \\
\hline
\end{tabular}

Tabla 4.14.-Variación de los Índices de Calidad en función de la duración media de los ciclos de buen y mal tiempo, $N$ y $S$, para la red mixta.

\section{Escenario CAL-4}

En este escenario evaluaremos la influencia relativa de los distintos elementos que componen cada red en sus índices de calidad. Este estudio sensitivo se realizó evaluando la influencia que la modificación de las tasa de fallo y tiempos de reparación de los componentes más significativos (líneas aéreas, cables subterráneos, interruptores automáticos, seccionadores, seccionadores-fusibles, ruptofusibles y transformadores) ejerce sobre los índices de Calidad de Servicio. 
Se utilizan, como características de fiabilidad base de los elementos de la red, los valores de las tasas de fallo y tiempos de reparación que aparecen en el Anexo 2. Los valores de cada elemento se decrementarán e incrementarán de forma individual para analizar las variaciones que provocan en los índices de calidad de la red. Las condiciones que impondremos a nuestra simulación son las expuestas en el apartado 3.1.1.

Para poder obtener la influencia de cada componente en los índices de calidad de la red, variaremos conjuntamente los valores de las tasas de fallo y tiempos de reparación de cada componente, obteniendo cinco grupos de valores distintos para cada una de las tres redes de ensayo. Desde la Tabla 4.15 a la 4.19 se pueden ver los resultados obtenidos para la red aérea, de la Tabla 4.20 a la 4.24 los resultados para la red subterránea y las Tablas 4.25 y 4.26 los de la red mixta.

\begin{tabular}{|c|c|c|c|c|c|}
\cline { 2 - 6 } \multicolumn{1}{c|}{} & \multicolumn{5}{c|}{ Tasas de Fallo y Tiempos de Reparación de las LA } \\
\hline Índices & $\begin{array}{c}\text { Caso Base } \\
\text { x 0,25 }\end{array}$ & $\begin{array}{c}\text { Caso Base } \\
\text { x 0,5 }\end{array}$ & Caso Base & $\begin{array}{c}\text { Caso Base } \\
\text { x 2 }\end{array}$ & $\begin{array}{c}\text { Caso Base } \\
\text { x 4 }\end{array}$ \\
\hline TIEPI & 2,196223 & 3,683208 & 8,111493 & 21,49376 & 66,81124 \\
\hline TIEB & 2,142744 & 3,604830 & 7,965488 & 21,23515 & 65,94976 \\
\hline NIEPI & 0,663851 & 0,966418 & 1,582885 & 2,762500 & 5,008999 \\
\hline NIEB & 0,648629 & 0,946615 & 1,548378 & 2,706378 & 4,903435 \\
\hline IIS & 0,250710 & 0,420458 & 0,925970 & 2,453626 & 7,626854 \\
\hline ENS & 2,951724 & 4,950231 & 10,90184 & 28,88761 & 89,79430 \\
\hline K & 11,67532 & 17,03907 & 27,87081 & 48,71480 & 88,26181 \\
\hline
\end{tabular}

Tabla 4.15.-Variación de los Índices de Calidad de la red aérea en función de las tasas de fallos y tiempos de reparación de las líneas aéreas.

\begin{tabular}{|c|c|c|c|c|c|}
\cline { 2 - 6 } \multicolumn{1}{c|}{} & \multicolumn{5}{c|}{ Tasas de Fallo y Tiempos de Reparación de los XS } \\
\hline Índices & $\begin{array}{c}\text { Caso Base } \\
\text { x 0,25 }\end{array}$ & $\begin{array}{c}\text { Caso Base } \\
\text { x 0,5 }\end{array}$ & Caso Base & $\begin{array}{c}\text { Caso Base } \\
\text { x 2 }\end{array}$ & $\begin{array}{c}\text { Caso Base } \\
\text { x 4 }\end{array}$ \\
\hline TIEPI & 7,834835 & 7,848564 & 8,111493 & 8,723268 & 9,940245 \\
\hline TIEB & 7,683689 & 7,724459 & 7,965488 & 8,560363 & 9,782344 \\
\hline NIEPI & 1,450734 & 1,484895 & 1,582885 & 1,776019 & 2,101842 \\
\hline NIEB & 1,421894 & 1,457250 & 1,548378 & 1,733385 & 2,058138 \\
\hline IIS & 0,894387 & 0,895955 & 0,925970 & 0,995807 & 1,134731 \\
\hline ENS & 10,53001 & 10,54847 & 10,90184 & 11,72407 & 13,35968 \\
\hline K & 25,59409 & 26,23050 & 27,87081 & 31,20093 & 37,04648 \\
\hline
\end{tabular}

Tabla 4.16.-Variación de los Índices de Calidad de la red aérea en función de las tasas de fallos y tiempos de reparación de los seccionadores-fusibles.

Tasas de Fallo y Tiempos de Reparación de los IA 


\begin{tabular}{|c|c|c|c|c|c|}
\hline Índices & $\begin{array}{c}\text { Caso Base } \\
\text { x 0,25 }\end{array}$ & $\begin{array}{c}\text { Caso Base } \\
\text { x 0,5 }\end{array}$ & Caso Base & $\begin{array}{c}\text { Caso Base } \\
\text { x 2 }\end{array}$ & $\begin{array}{c}\text { Caso Base } \\
\text { x 4 }\end{array}$ \\
\hline TIEPI & 8,134151 & 8,114395 & 8,111493 & 8,177643 & 8,125384 \\
\hline TIEB & 7,995382 & 7,991129 & 7,965488 & 8,022090 & 8,007697 \\
\hline NIEPI & 1,586128 & 1,586464 & 1,582885 & 1,596304 & 1,604947 \\
\hline NIEB & 1,555435 & 1,557285 & 1,548378 & 1,563806 & 1,572347 \\
\hline IIS & 0,928556 & 0,926301 & 0,925970 & 0,933521 & 0,927555 \\
\hline ENS & 10,93229 & 10,90574 & 10,90184 & 10,99075 & 10,92051 \\
\hline K & 27,99783 & 28,03112 & 27,87081 & 28,14850 & 28,30224 \\
\hline
\end{tabular}

Tabla 4.17.-Variación de los Índices de Calidad de la red aérea en función de las tasas de fallos y tiempos de reparación de los interruptores automáticos.

\begin{tabular}{|c|c|c|c|c|c|}
\cline { 2 - 6 } \multicolumn{1}{c|}{} & \multicolumn{5}{c|}{ Tasas de Fallo y Tiempos de Reparación de los TF } \\
\hline Índices & $\begin{array}{c}\text { Caso Base } \\
\text { x 0,25 }\end{array}$ & $\begin{array}{c}\text { Caso Base } \\
\text { x 0,5 }\end{array}$ & Caso Base & $\begin{array}{c}\text { Caso Base } \\
\text { x 2 }\end{array}$ & $\begin{array}{c}\text { Caso Base } \\
\text { x 4 }\end{array}$ \\
\hline TIEPI & 7,744428 & 7,782367 & 8,111493 & 9,387157 & 13,48901 \\
\hline TIEB & 7,586468 & 7,628189 & 7,965488 & 9,211749 & 13,38625 \\
\hline NIEPI & 1,535044 & 1,546622 & 1,582885 & 1,661516 & 1,769111 \\
\hline NIEB & 1,500588 & 1,510248 & 1,548378 & 1,625053 & 1,739006 \\
\hline IIS & 0,884067 & 0,888398 & 0,925970 & 1,071593 & 1,539842 \\
\hline ENS & 10,40851 & 10,45950 & 10,90184 & 12,61634 & 18,12923 \\
\hline K & 27,01058 & 27,18446 & 27,87081 & 29,25095 & 31,30211 \\
\hline
\end{tabular}

Tabla 4.18.-Variación de los Índices de Calidad de la red aérea en función de las tasas de fallos y tiempos de reparación de los transformadores de intemperie.

\begin{tabular}{|c|c|c|c|c|c|}
\cline { 2 - 6 } \multicolumn{1}{c|}{} & \multicolumn{5}{c|}{ Tasas de Fallo y Tiempos de Reparación de los SV } \\
\hline Índices & $\begin{array}{c}\text { Caso Base } \\
\text { x 0,25 }\end{array}$ & $\begin{array}{c}\text { Caso Base } \\
\text { x 0,5 }\end{array}$ & Caso Base & $\begin{array}{c}\text { Caso Base } \\
\text { x 2 }\end{array}$ & $\begin{array}{c}\text { Caso Base } \\
\text { x 4 }\end{array}$ \\
\hline TIEPI & 8,084787 & 8,168578 & 8,111493 & 8,186378 & 8,353454 \\
\hline TIEB & 7,925790 & 8,019451 & 7,965488 & 8,043840 & 8,177717 \\
\hline NIEPI & 1,561767 & 1,579391 & 1,582885 & 1,613407 & 1,667234 \\
\hline NIEB & 1,527720 & 1,543731 & 1,548378 & 1,579768 & 1,629847 \\
\hline IIS & 0,922921 & 0,932486 & 0,925970 & 0,934518 & 0,953591 \\
\hline ENS & 10,86595 & 10,97856 & 10,90184 & 11,00249 & 11,22704 \\
\hline K & 27,49895 & 27,78715 & 27,87081 & 28,43583 & 29,33724 \\
\hline
\end{tabular}

Tabla 4.19.-Variación de los Índices de la red aérea Calidad en función de las tasas de fallos y tiempos de reparación de los seccionadores de intemperie. 
$\mathrm{Si}$, para una mejor apreciación de estos resultados, representamos estas variaciones de los índices de calidad, por ejemplo para el TIEPI, NIEPI, y ENS (véase figuras 4.5, 4.6 y 4.7), en función de las variaciones de las distintas tasas de fallo y tiempos de reparación, se observa claramente, que son los fallos en las líneas aéreas los que contribuyen decisivamente en el incremento de dichos índices. Merece la pena, también, destacar la pequeñísima influencia de los interruptores automáticos (en este caso, la red de ensayo aérea, sólo tiene el IA de cabecera) que, aunque se halla presente en los caminos mínimos de todos los puntos de carga, posee, lo que es característico, una tasa de fallo muy pequeña y un tiempo de reposición prácticamente despreciable.

Podríamos concluir así, que una mejora en la calidad de los elementos que no recayera sobre las líneas aéreas, supondría la realización de una gran inversión (especialmente en el caso de los transformadores de potencia y de los interruptores automáticos), que apenas mejoraría la calidad del servicio prestado por la compañía eléctrica.

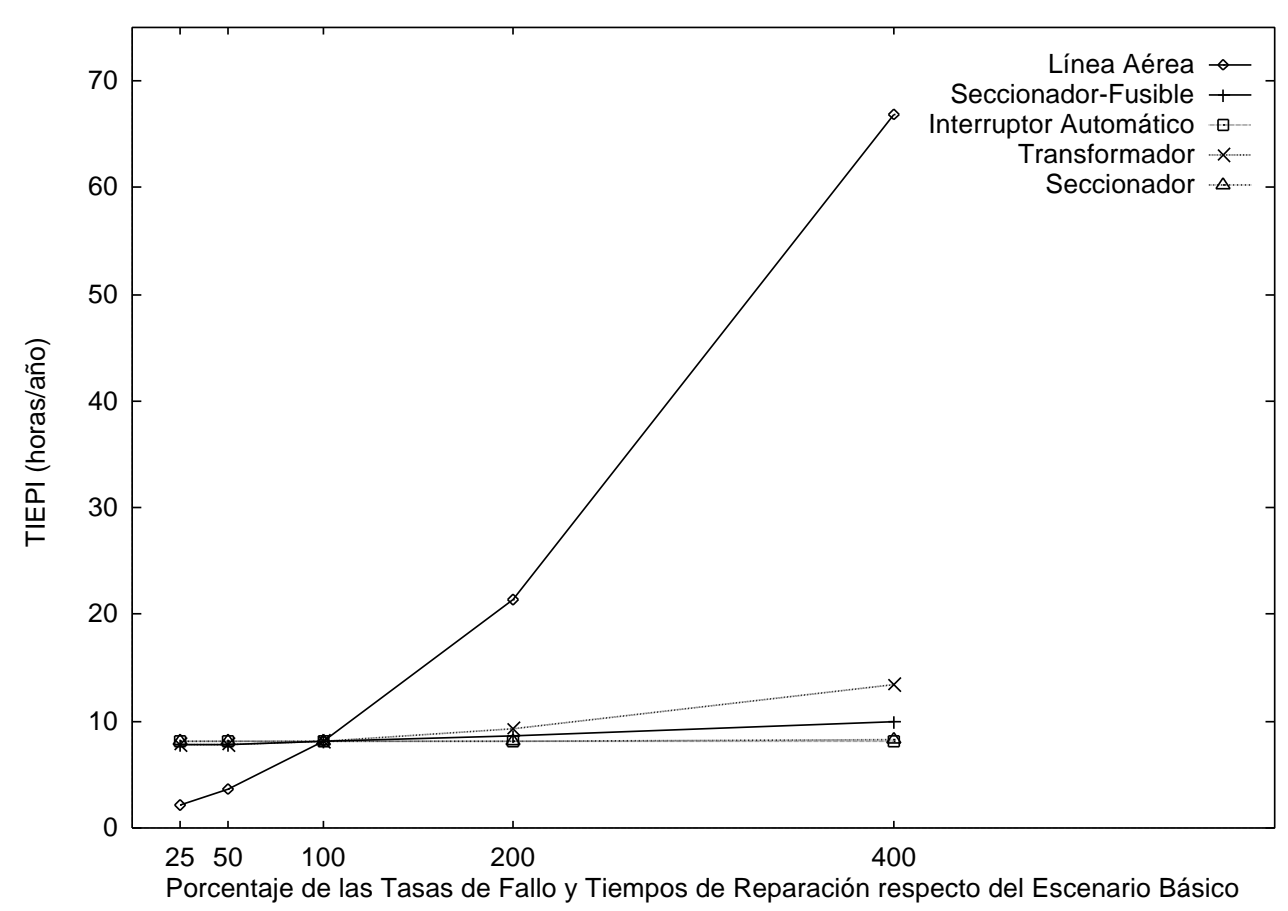

Fig.4.5.-Variación del TIEPI con las tasas de fallo y tiempos de reparación de los principales elementos que componen la red aérea. 


\section{CAPÍTULO 4 - RESULTADOS}

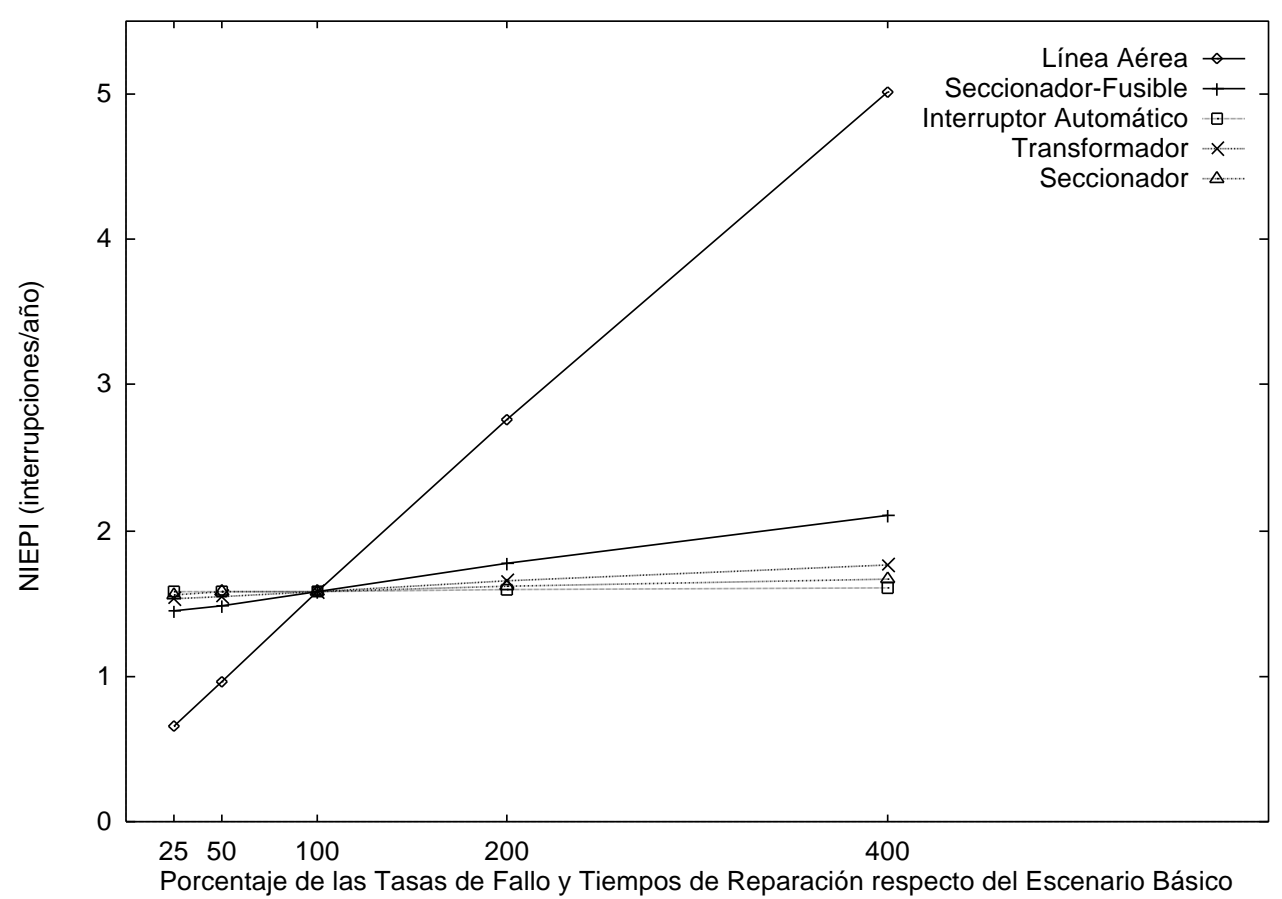

Fig.4.6.-Variación del NIEPI con las tasas de fallo y tiempos de reparación de los principales elementos que componen la red aérea.

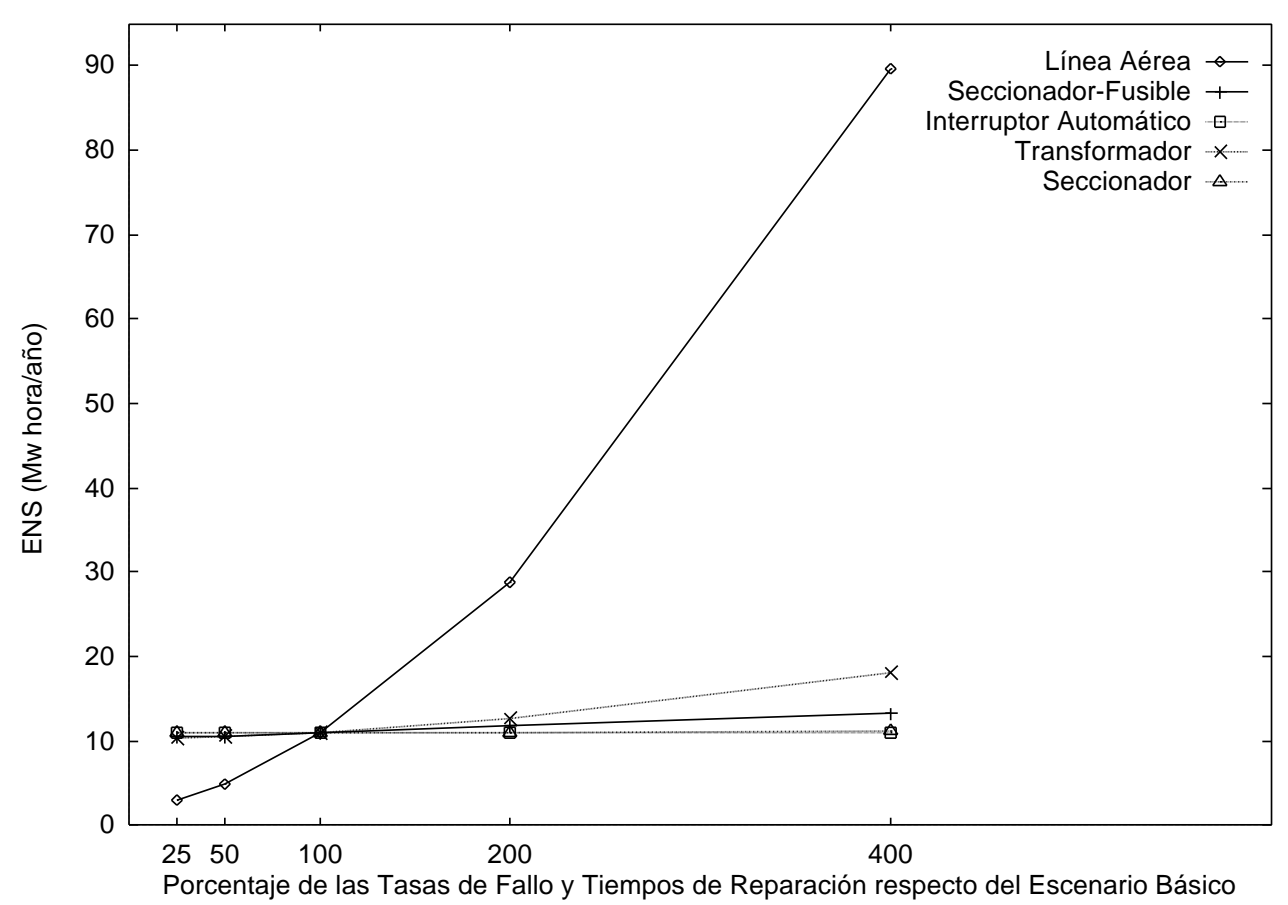

Fig.4.7.-Variación del ENS con las tasas de fallo y tiempos de reparación de los principales elementos que componen la red aérea. 
En las figuras 4.8, 4.9 y 4.10 presentaremos alguno de los resultados de las tablas 4.20 a 4.24. En particular, representamos las variaciones de los índices de calidad TIEPI, NIEPI, y ENS de la red subterránea en función de las variaciones de las distintas tasas de fallo y tiempos de reparación. Se aprecia que la influencia de los cables subterráneos, no es tan acusada como la de las líneas aéreas en el caso anterior. Evidentemente, la tasa global de fallo en los cables subterráneos es menor que en las líneas aéreas y por tanto contribuye en menor porcentaje a la indisponibilidad total. Esta influencia de los cables subterráneos es más evidente en los índices relacionados con la duración de las interrupciones que en los relacionados con el número de ellas, debido a su elevado tiempo de reposición en comparación con el resto de los elementos (véase Tabla A2-3 en el Anexo 2).

También, es destacable la gran influencia de los ruptofusibles sobre el número de interrupciones aunque no sobre su duración, ya que las interrupciones que estos provocan se reponen mucho más rápidamente que las provocadas por los cables subterráneos (1 hora, frente a las 20 horas de los cables).

\begin{tabular}{|c|c|c|c|c|c|}
\cline { 2 - 6 } \multicolumn{1}{c|}{} & \multicolumn{5}{c|}{ Tasas de Fallo y Tiempos de Reparación de los CS } \\
\hline Índices & $\begin{array}{c}\text { Caso Base } \\
\text { x 0,25 }\end{array}$ & $\begin{array}{c}\text { Caso Base } \\
\text { x 0,5 }\end{array}$ & Caso Base & $\begin{array}{c}\text { Caso Base } \\
\text { x 2 }\end{array}$ & $\begin{array}{c}\text { Caso Base } \\
\text { x 4 }\end{array}$ \\
\hline TIEPI & 0,878157 & 1,234954 & 2,536917 & 7,573732 & 27,63561 \\
\hline TIEB & 0,883802 & 1,238752 & 2,552843 & 7,598553 & 27,82988 \\
\hline NIEPI & 0,304156 & 0,331319 & 0,385004 & 0,492079 & 0,704123 \\
\hline NIEB & 0,305242 & 0,332054 & 0,385310 & 0,491477 & 0,703411 \\
\hline IIS & 0,100246 & 0,140976 & 0,289602 & 0,864581 & 3,154751 \\
\hline ENS & 7,307145 & 10,27605 & 21,10968 & 63,02103 & 229,9559 \\
\hline K & 8,852004 & 9,629560 & 11,17399 & 14,25282 & 20,39893 \\
\hline
\end{tabular}

Tabla 4.20.-Variación de los Índices de Calidad de la red subterránea en función de las tasas de fallos y tiempos de reparación de los cables subterráneos.

\begin{tabular}{|c|c|c|c|c|c|}
\cline { 2 - 6 } \multicolumn{1}{c|}{} & \multicolumn{5}{c|}{ Tasas de Fallo y Tiempos de Reparación de los RF } \\
\hline Índices & $\begin{array}{c}\text { Caso Base } \\
\text { x 0,25 }\end{array}$ & $\begin{array}{c}\text { Caso Base } \\
\text { x 0,5 }\end{array}$ & Caso Base & $\begin{array}{c}\text { Caso Base } \\
\text { x 2 }\end{array}$ & $\begin{array}{c}\text { Caso Base } \\
\text { x 4 }\end{array}$ \\
\hline TIEPI & 2,441107 & 2,462208 & 2,536917 & 2,712610 & 3,050876 \\
\hline TIEB & 2,452965 & 2,473364 & 2,552843 & 2,717544 & 3,061532 \\
\hline NIEPI & 0,316145 & 0,338248 & 0,385004 & 0,477480 & 0,663578 \\
\hline NIEB & 0,316740 & 0,338917 & 0,385310 & 0,477089 & 0,662652 \\
\hline IIS & 0,278665 & 0,281074 & 0,289602 & 0,309659 & 0,348273 \\
\hline ENS & 20,31245 & 20,48803 & 21,10968 & 22,57162 & 25,38633 \\
\hline K & 9,185473 & 9,828588 & 11,17399 & 13,83558 & 19,21690 \\
\hline
\end{tabular}

Tabla 4.21.-Variación de los Índices de Calidad de la red subterránea en función de las tasas de fallos y tiempos de reparación de los ruptofusibles. 


\begin{tabular}{|c|c|c|c|c|c|}
\cline { 2 - 6 } \multicolumn{1}{c|}{} & \multicolumn{5}{c|}{ Tasas de Fallo y Tiempos de Reparación de los IA } \\
\hline Índices & $\begin{array}{c}\text { Caso Base } \\
\text { x 0,25 }\end{array}$ & $\begin{array}{c}\text { Caso Base } \\
\text { x 0,5 }\end{array}$ & Caso Base & $\begin{array}{c}\text { Caso Base } \\
\text { x 2 }\end{array}$ & $\begin{array}{c}\text { Caso Base } \\
\text { x 4 }\end{array}$ \\
\hline TIEPI & 2,501491 & 2,501695 & 2,536917 & 2,698663 & 3,204815 \\
\hline TIEB & 2,510547 & 2,513240 & 2,552843 & 2,712725 & 3,221184 \\
\hline NIEPI & 0,369744 & 0,374969 & 0,385004 & 0,406340 & 0,447321 \\
\hline NIEB & 0,369933 & 0,375250 & 0,385310 & 0,407189 & 0,447696 \\
\hline IIS & 0,285558 & 0,285582 & 0,289602 & 0,308067 & 0,365846 \\
\hline ENS & 20,81490 & 20,81660 & 21,10968 & 22,45558 & 26,66726 \\
\hline K & 10,72806 & 10,88224 & 11,17399 & 11,80847 & 12,98319 \\
\hline
\end{tabular}

Tabla 4.22.-Variación de los Índices de Calidad de la red subterránea en función de las tasas de fallos y tiempos de reparación de los interruptores automáticos.

\begin{tabular}{|c|c|c|c|c|c|}
\cline { 2 - 6 } \multicolumn{1}{c|}{} & \multicolumn{5}{c|}{ Tasas de Fallo y Tiempos de Reparación de los TF } \\
\hline Índices & $\begin{array}{c}\text { Caso Base } \\
\text { x 0,25 }\end{array}$ & $\begin{array}{c}\text { Caso Base } \\
\text { x 0,5 }\end{array}$ & Caso Base & $\begin{array}{c}\text { Caso Base } \\
\text { x 2 }\end{array}$ & $\begin{array}{c}\text { Caso Base } \\
\text { x 4 }\end{array}$ \\
\hline TIEPI & 2,500754 & 2,539356 & 2,536917 & 2,648197 & 3,064109 \\
\hline TIEB & 2,509993 & 2,553638 & 2,552843 & 2,659775 & 3,080984 \\
\hline NIEPI & 0,380277 & 0,382392 & 0,385004 & 0,391630 & 0,404829 \\
\hline NIEB & 0,381009 & 0,382880 & 0,385310 & 0,391943 & 0,405508 \\
\hline IIS & 0,285474 & 0,289881 & 0,289602 & 0,302306 & 0,349784 \\
\hline ENS & 20,80877 & 21,12998 & 21,10968 & 22,03564 & 25,49645 \\
\hline K & 11,04925 & 11,10351 & 11,17399 & 11,36636 & 11,75973 \\
\hline
\end{tabular}

Tabla 4.23.-Variación de los Índices de Calidad de la red subterránea en función de las tasas de fallos y tiempos de reparación de los transformadores de interior.

\begin{tabular}{|c|c|c|c|c|c|}
\cline { 2 - 6 } \multicolumn{1}{c|}{} & \multicolumn{5}{c|}{ Tasas de Fallo y Tiempos de Reparación de los SV } \\
\hline Índices & $\begin{array}{c}\text { Caso Base } \\
\text { x 0,25 }\end{array}$ & $\begin{array}{c}\text { Caso Base } \\
\text { x 0,5 }\end{array}$ & Caso Base & $\begin{array}{c}\text { Caso Base } \\
\text { x 2 }\end{array}$ & $\begin{array}{c}\text { Caso Base } \\
\text { x 4 }\end{array}$ \\
\hline TIEPI & 2,450069 & 2,469390 & 2,536917 & 2,701516 & 2,973430 \\
\hline TIEB & 2,460634 & 2,483237 & 2,552843 & 2,718587 & 2,994383 \\
\hline NIEPI & 0,341671 & 0,358061 & 0,385004 & 0,443576 & 0,557447 \\
\hline NIEB & 0,341389 & 0,357035 & 0,385310 & 0,445006 & 0,561093 \\
\hline IIS & 0,279688 & 0,281894 & 0,289602 & 0,308392 & 0,339433 \\
\hline ENS & 20,38702 & 20,54780 & 21,10968 & 22,47931 & 24,74191 \\
\hline K & 9,900290 & 10,35401 & 11,17399 & 12,90518 & 16,27170 \\
\hline
\end{tabular}

Tabla 4.24.-Variación de los Índices de Calidad de la red subterránea en función de las tasas de fallos y tiempos de reparación de los seccionadores de interior. 
CAPÍTULO 4 - RESULTADOS

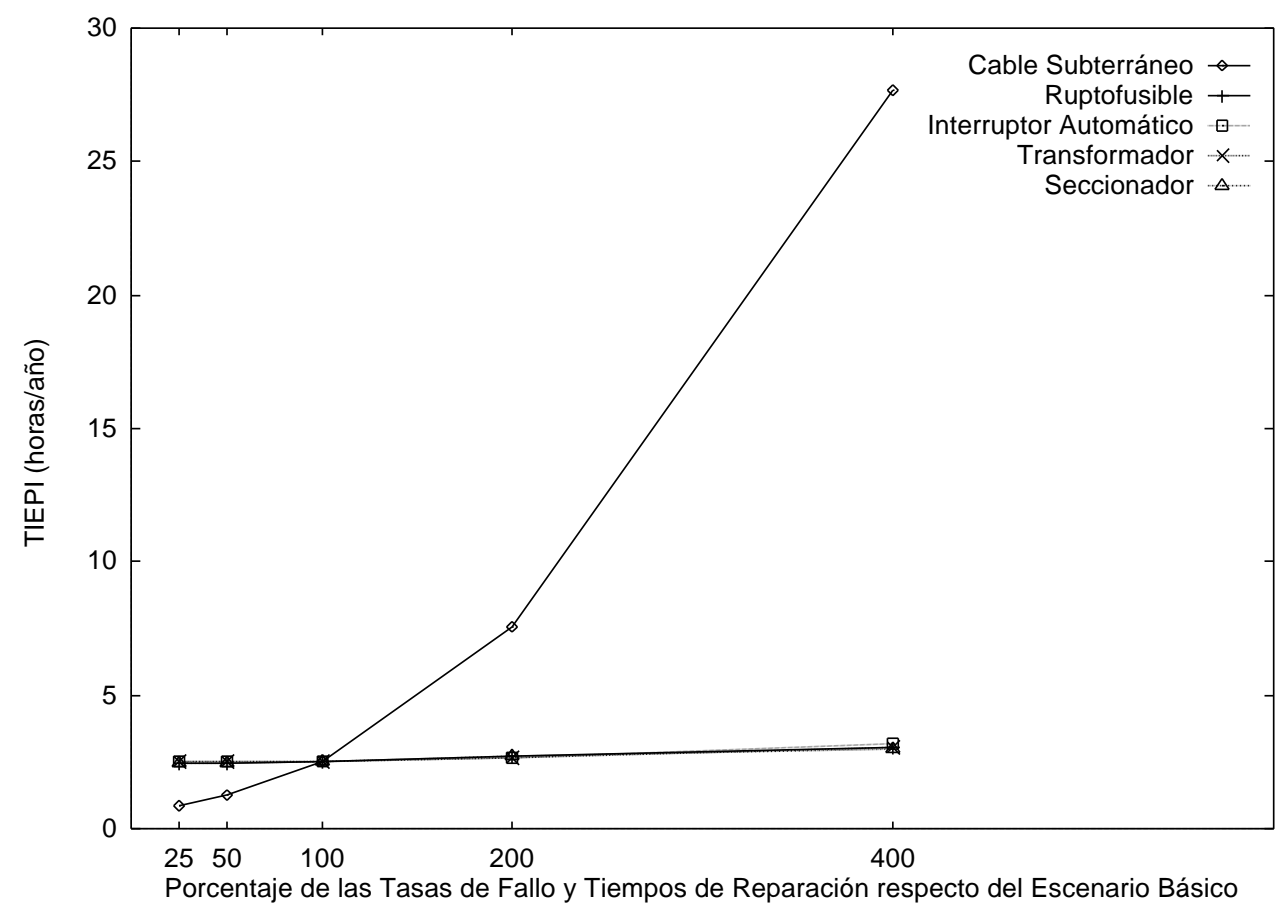

Fig.4.8.-Variación del TIEPI con las tasas de fallo y tiempos de reparación de los principales elementos que componen la red subterránea.

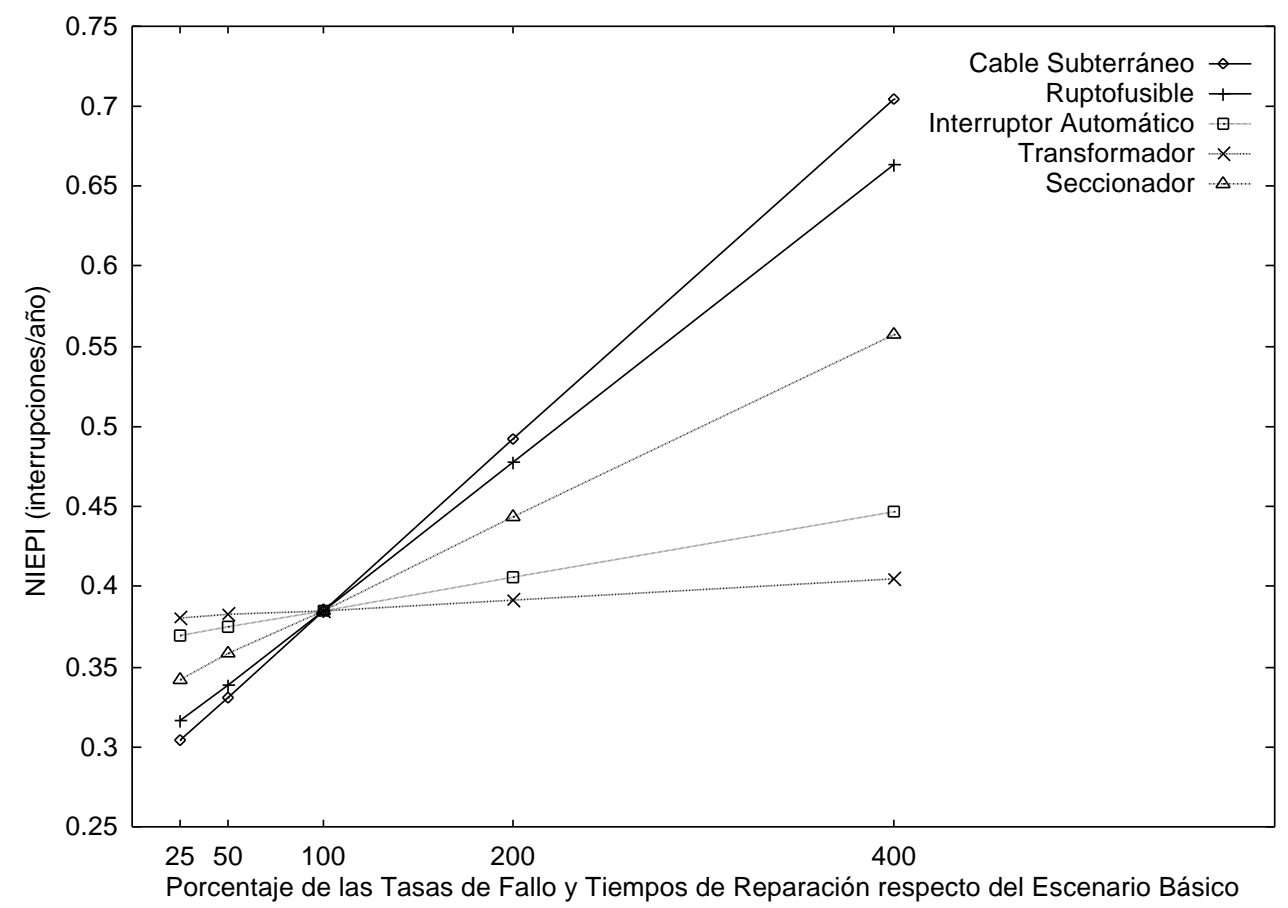

Fig.4.9.-Variación del NIEPI con las tasas de fallo y tiempos de reparación de los principales elementos que componen la red subterránea. 


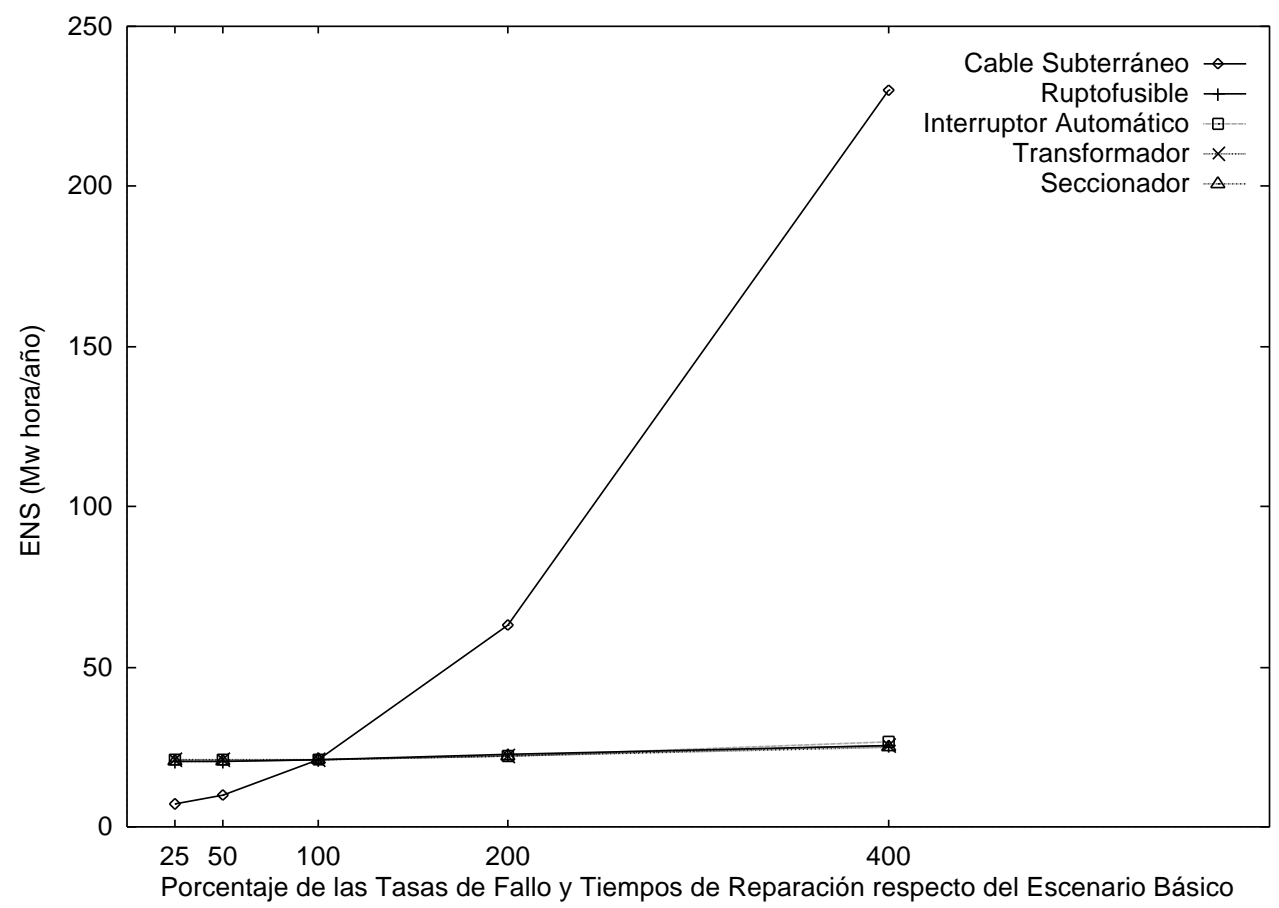

Fig.4.10.-Variación del ENS con las tasas de fallo y tiempos de reparación de los principales elementos que componen la red subterránea.

En las figuras $4.11,4.12$ y 4.13 presentaremos alguno de los resultados recogidos en las tablas 4.25 y 4.26. En particular, representamos las variaciones de los índices de calidad TIEPI, NIEPI, y ENS de la red mixta en función de la variación, conjunta, de las distintas tasas de fallo y tiempos de reparación, de las líneas aéreas y cables subterráneos presentes en esta red. Se han elegido, solamente, estos dos elementos a la vista de los resultados obtenidos en la red aérea y subterránea, en los que se constató que eran estos, los elementos que más influencia ejercen sobre la indisponibilidad de estas redes.

Par la red mixta, se aprecia que la influencia de los cables subterráneos en la indisponibilidad de la red, no es tan acusada como la de las líneas aéreas. Por tanto, en orden a la mejora de la calidad de servicio en la red mixta, sería preferente una inversión encaminada a mejorar la calidad de las líneas aéreas que a cualquier otro elemento.

Este resultado es válido tanto en los índices relacionados con el número de interrupciones como en los relacionados con la duración de éstas. No obstante, la influencia de las líneas aéreas es superior en los índices relacionados con el número de interrupciones que en los relacionados con la duración de éstas, debido a que el tiempo de reposición de los cables subterráneos es mayor. 


\begin{tabular}{|c|c|c|c|c|c|}
\cline { 2 - 6 } \multicolumn{1}{c|}{} & \multicolumn{5}{c|}{ Tasas de Fallo y Tiempos de Reparación de las LA } \\
\hline Índices & $\begin{array}{c}\text { Caso Base } \\
\text { x 0,25 }\end{array}$ & $\begin{array}{c}\text { Caso Base } \\
\text { x 0,5 }\end{array}$ & Caso Base & $\begin{array}{c}\text { Caso Base } \\
\text { x 2 }\end{array}$ & $\begin{array}{c}\text { Caso Base } \\
\text { x 4 }\end{array}$ \\
\hline TIEPI & 2,738243 & 3,934427 & 7,359767 & 18,58852 & 57,61607 \\
\hline TIEB & 2,536684 & 3,758940 & 7,282107 & 18,66678 & 57,94295 \\
\hline NIEPI & 0,676825 & 0,897548 & 1,328792 & 2,179833 & 3,798093 \\
\hline NIEB & 0,654676 & 0,884730 & 1,342351 & 2,232471 & 3,931102 \\
\hline IIS & 0,312585 & 0,449135 & 0,840156 & 2,121977 & 6,577178 \\
\hline ENS & 15,41356 & 22,14688 & 41,42812 & 104,6347 & 324,3207 \\
\hline K & 38,62590 & 52,19908 & 79,19871 & 131,7157 & 231,9349 \\
\hline
\end{tabular}

Tabla 4.25.-Variación de los Índices de Calidad de la red mixta en función de las tasas de fallos y tiempos de reparación de las líneas aéreas.

\begin{tabular}{|c|c|c|c|c|c|}
\cline { 2 - 6 } \multicolumn{1}{c|}{} & \multicolumn{5}{c|}{ Tasas de Fallo y Tiempos de Reparación de los CS } \\
\hline Índices & $\begin{array}{c}\text { Caso Base } \\
\text { x 0,25 }\end{array}$ & $\begin{array}{c}\text { Caso Base } \\
\text { x 0,5 }\end{array}$ & Caso Base & $\begin{array}{c}\text { Caso Base } \\
\text { x 2 }\end{array}$ & $\begin{array}{c}\text { Caso Base } \\
\text { x 4 }\end{array}$ \\
\hline TIEPI & 6,460016 & 6,695227 & 7,359767 & 10,08064 & 21,36843 \\
\hline TIEB & 6,596543 & 6,778438 & 7,282107 & 9,384855 & 17,78705 \\
\hline NIEPI & 1,285647 & 1,305241 & 1,328792 & 1,385350 & 1,517734 \\
\hline NIEB & 1,306421 & 1,319880 & 1,342351 & 1,384912 & 1,482912 \\
\hline IIS & 0,737445 & 0,764295 & 0,840156 & 1,150759 & 2,439319 \\
\hline ENS & 36,36341 & 37,68742 & 41,42812 & 56,74395 & 120,2828 \\
\hline K & 77,07881 & 77,87287 & 79,19871 & 81,70978 & 87,49182 \\
\hline
\end{tabular}

Tabla 4.26.-Variación de los Índices de Calidad de la red mixta en función de las tasas de fallos y tiempos de reparación de los cables subterráneos. 


\section{CAPÍTULO 4 - RESULTADOS}

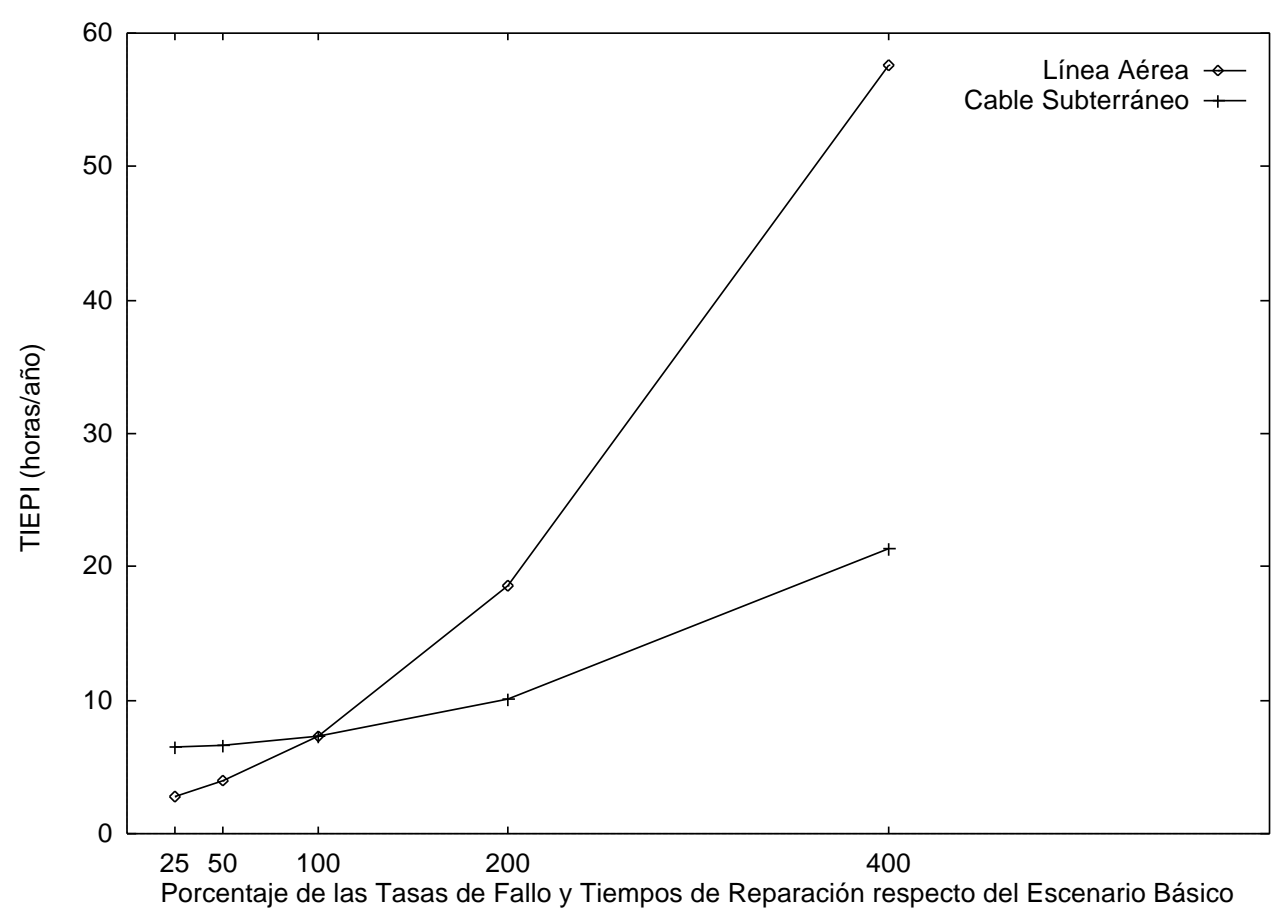

Fig.4.11.-Variación del TIEPI con las tasas de fallo y tiempos de reparación de los principales elementos que componen la red mixta.

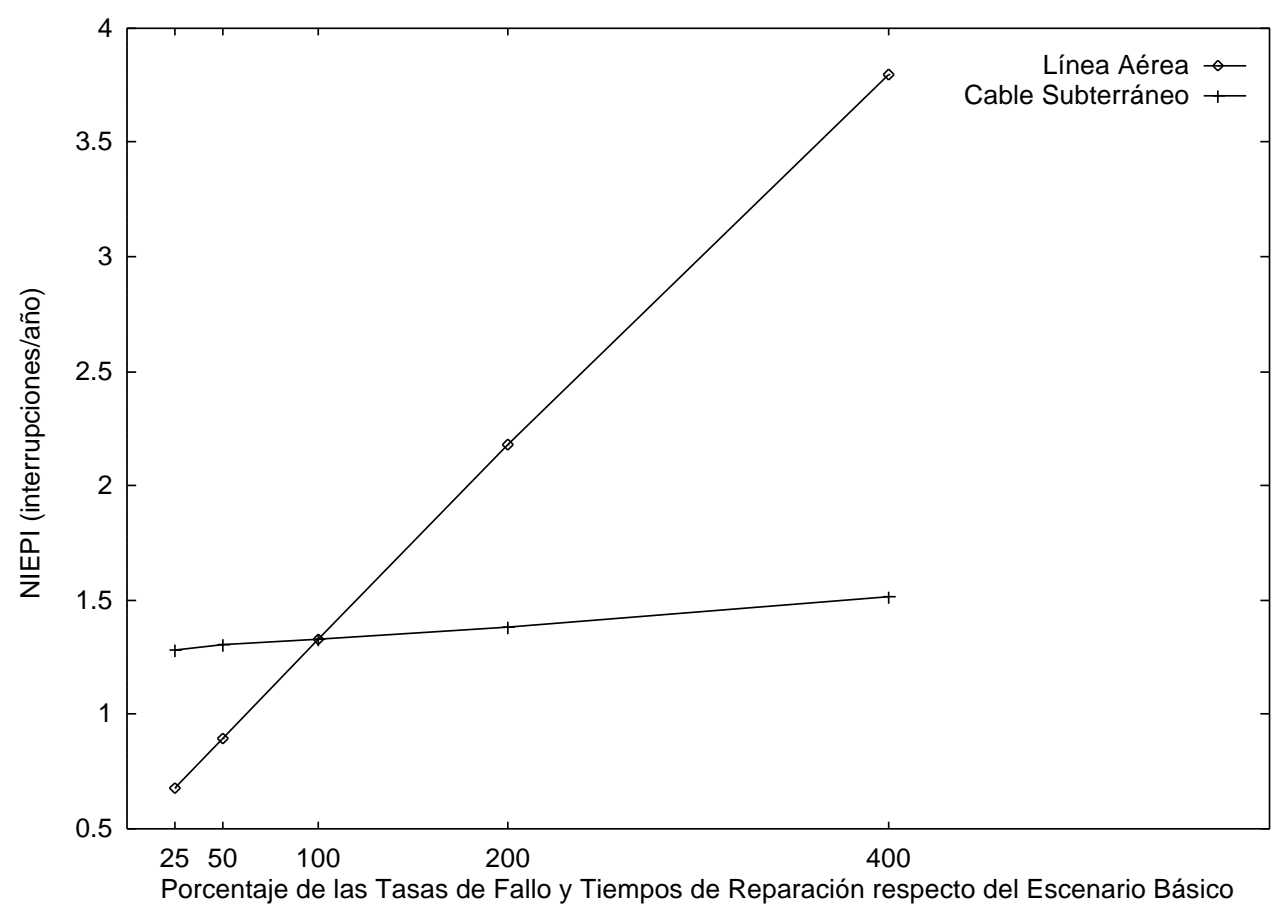

Fig.4.12.-Variación del NIEPI con las tasas de fallo y tiempos de reparación de los principales elementos que componen la red mixta. 


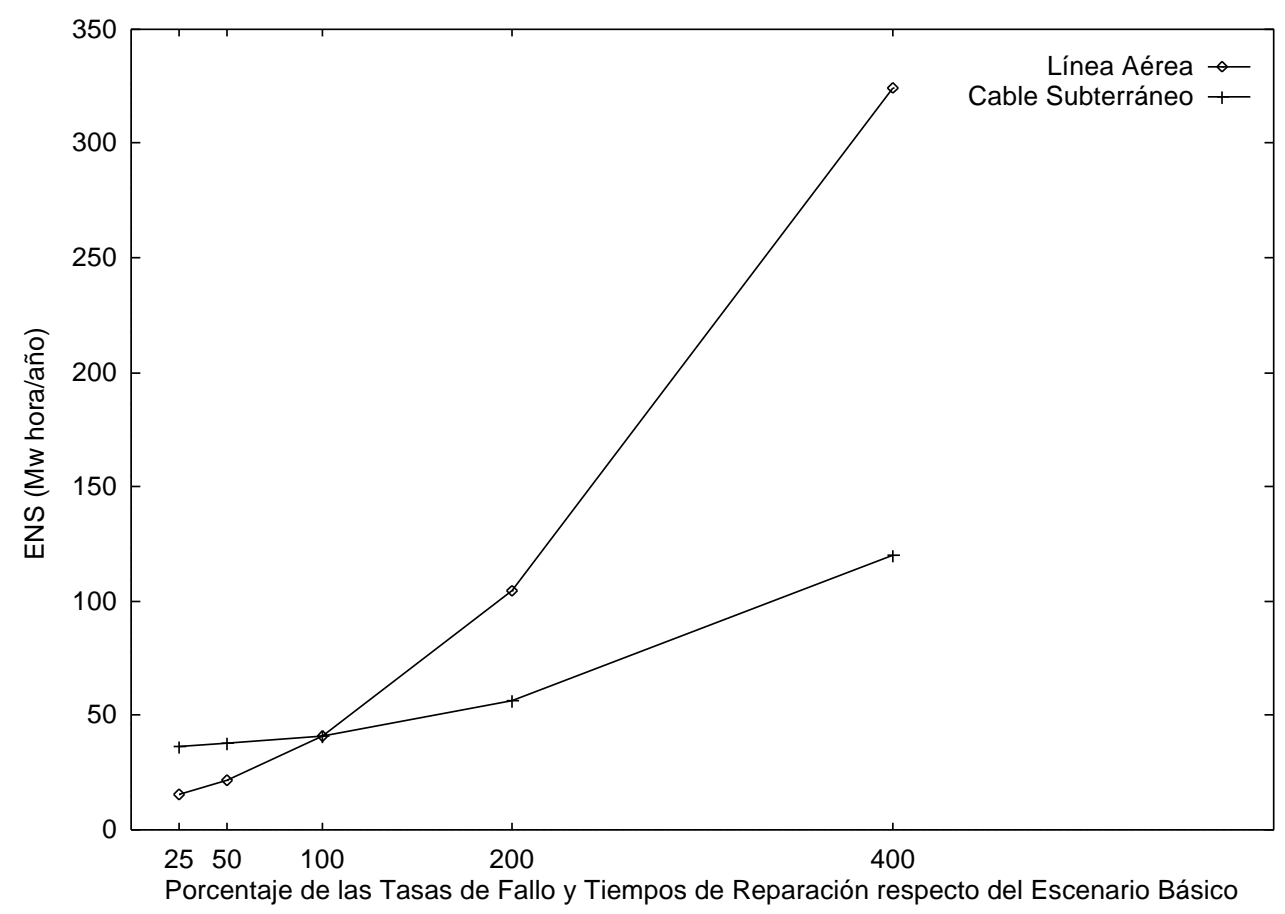

Fig.4.13.-Variación del ENS con las tasas de fallo y tiempos de reparación de los principales elementos que componen la red mixta.

\section{ASPECTOS A DESTACAR SOBRE LOS ÍNDICES DE CALIDAD}

Se ha encontrado que las hipótesis simplificadoras que, normalmente, se hacen en la técnica basada en los procesos de Markov, para la obtención de los índices de calidad de la red, son bastante razonables. No obstante, nuestro proceso de simulación, se muestra superior ya que tiene en cuenta los fallos que provocan interrupciones cuya duración se solapa.

Hemos constatado que los índices de calidad no dependen apreciablemente del tipo de distribución escogida (exponencial o normal truncada) para el tiempo de reparación de los elementos que forman las redes.

Se ha encontrado que disminuyendo el tiempo medio de localización y desplazamiento se aumenta linealmente el valor de los índices de calidad de una red.

Por último, en relación con la calidad de servicio de cualquiera de las tres redes ensayadas, se ha hallado que sería preferible una inversión encaminada a disminuir la tasa de fallo y/o tiempo de reparación de las líneas aéreas que, una actuación equivalente sobre cualquier otro elemento.

\subsection{DISTRIBUCIONES Y SU COMPORTAMIENTO}


Los efectos que, para un consumidor, se derivan de la fiabilidad de una red, inciden en él a través del punto de carga, no distinguiendo entre los diversos elementos que forman la línea sino asimilándolos a un todo. Sería pues, importante reducir todas las secuencias de ciclos de funcionamiento-fallo de todos los elementos que transmiten la energía desde la subestación al punto de carga, a una sola secuencia que representaría los ciclos de disponibilidad-indisponibilidad del punto de carga analizado. A partir de esta secuencia equivalente se hallarían las distribuciones del "Tiempo hasta la Interrupción" y del "Tiempo de Reposición de Servicio" o "Tiempo de Indisponibilidad" de cada punto de carga.

Para estudiar correctamente la fiabilidad de una red eléctrica, no sólo es necesario obtener los índices de calidad asociados a los puntos de carga del sistema; es muy importante, también, obtener la distribución de frecuencias del "Tiempo hasta la Interrupción" y del "Tiempo de Reposición de Servicio" o "Tiempo de Indisponibilidad" de cada uno de ellos y, si fuera posible, parametrizar cada una de las distribuciones asociadas a cada punto de carga.

Existen varias razones que ahondan en la necesidad de obtener y estudiar la distribución de frecuencias del "Tiempo hasta la Interrupción" y del "Tiempo de Indisponibilidad" de cada punto de carga: A saber:

- Si la distribución de frecuencias tiene algún valor anormalmente grande para tiempos hasta la interrupción pequeños, puede ayudarnos a diagnosticar algún elemento que sería preciso proteger para que no falle tan a menudo o substituirlo por uno que no falle tanto.

- Deben conocerse la duraciones límite, superior e inferior, de la distribución de tiempo hasta la interrupción.

- Es importante conocer el límite superior de la duración de las interrupciones, pues todas las exigencias que el consumidor plantea al servicio no merecen el mismo tratamiento. Un ejemplo lo representa la existencia de algún límite de duración de interrupción que no se pueda sobrepasar. Ésta sería la única forma de averiguarlo. Repercute, indudablemente, en la evaluación de estrategias para mejorar la fiabilidad por parte de la compañía eléctrica.

- Si la distribución de frecuencias tiene algún valor anormalmente grande lejos de media, puede ayudarnos a diagnosticar algún elemento que sería preciso proteger especialmente para evitar tiempos de reposición elevados.

También, es importante encontrar el tipo de distribución estadística que sigue el "Tiempo hasta la Interrupción" y el "Tiempo de Indisponibilidad" de la secuencia equivalente de ciclos de funcionamiento-fallo de cada punto de carga que componen una red. Una vez obtenidas estas distribuciones y convenientemente parametrizadas dispondremos de dos ventajas principales:

- Si se tiene la distribución estadística teórica que sigue una distribución experimental, es muy sencillo calcular cualquier índice asociado con una distribución de probabilidad, como la probabilidad de que la duración de una 
interrupción sea menor que una de valor dado o la probabilidad de que se llegue a un tiempo dado sin que el servicio se halla interrumpido.

- Para obtener el ciclo de funcionamiento-fallo de cada punto de carga ya no necesitaríamos simular el funcionamiento de todos los elementos del punto de carga para luego sumarlos; bastaría con utilizar estas dos distribuciones asociadas a cada punto de carga como si de la simulación de un único elemento se tratase. Esto incrementaría la velocidad de computo para posteriores simulaciones en las que buscásemos otros resultados derivados de los ciclos funcionamiento-fallo de los puntos de carga de la red, y asociados, por ejemplo, a los costos que los fallos de la red provocan en el consumidor (A_BILLCHA-94) o en la compañía eléctrica (L_IEEE-91).

Utilizaremos los diversos escenarios, definidos en el apartado 3.1.2, para una red aérea, una red subterránea y una red mixta, analizando, en primer lugar, el comportamiento de las distribuciones de frecuencias del "tiempo hasta la interrupción" y del "tiempo de reposición de servicio" de sus puntos de carga, para luego parametrizar las mediante distribuciones de probabilidad conocidas.

\subsubsection{RED SUBTERRÁNEA}

Como se ha anunciado, este análisis se centrará en el efecto que la variación de la tasa de fallo y tiempos de reparación de uno o dos elementos del sistema tiene sobre las indisponibilidades de los puntos de carga. Para ello contamos con cuatro escenarios que se utilizarán en dos ocasiones, una, suponiendo que la hipótesis de distribución de los tiempos de reparación de los elementos de la red sigue una distribución exponencial, y, otra, una normal truncada.

Para representar los resultados hemos elegido, véase figura 3.2, unas veces, el punto de carga número " 0 ", que se halla en el nudo de carga "7" en el centro de transformación $1^{\circ}$ (CT1), y otras, el número "10", situado en el nudo de carga número "48" en el centro de transformación $6^{\circ}$ (CT6). No existe ninguna razón especial para esta elección, y podríamos haber elegido otros cualesquiera ya que los restantes puntos de carga se comportan cualitativamente de la misma forma que estos dos. Para localizar cualquier otro punto de carga en el esquema topológico de la figura 3.2, únicamente, deberemos ir a la tabla con los datos topológicos de los puntos carga que se halla en el Anexo 3 y ver a que número de nudo corresponde el número de orden del punto de carga que queremos localizar. 


\section{DISTRIBUCIÓN EXPONENCIAL PARA EL TIEMPO DE REPARACIÓN (DETR) DE LOS ELEMENTOS DE LA RED.}

\section{Escenario SUB-1 (DETR)}

Los resultados, de la distribución del tiempo de indisponibilidad para el punto de carga número "0", se recogen en la figura 4.14, donde se aprecia que la mayoría de las interrupciones fueron subsanadas en un tiempo por debajo de las 10 horas, aunque hay un pequeño grupo de ellas que abarcan un espectro comprendido entre este valor y, casi, las 127 horas, que es el valor límite de duración de una interrupción para el punto de carga "0".

La distribución del tiempo de indisponibilidad de la figura 4.14 puede ajustarse mediante una función del tipo exponencial:

$$
f(x)=\frac{b}{x^{2}} e^{a-\frac{b}{x}}
$$

como puede comprobarse sobre la figura 4.15.

La conclusión es general para cualquier punto de carga.

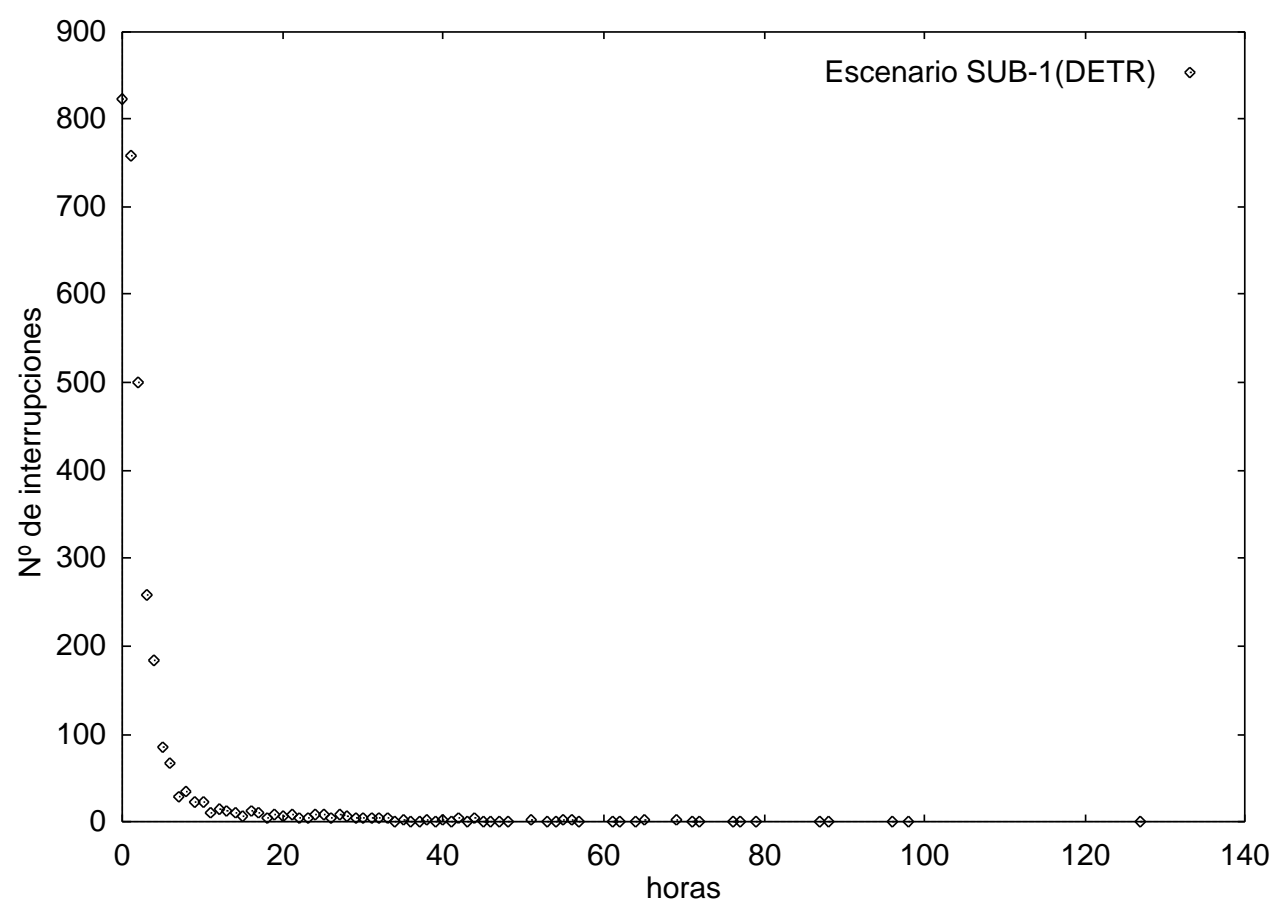

Fig.4.14.-Distribución de tiempo de indisponibilidad para el punto de carga número "0" de la red subterránea para el caso básico. 


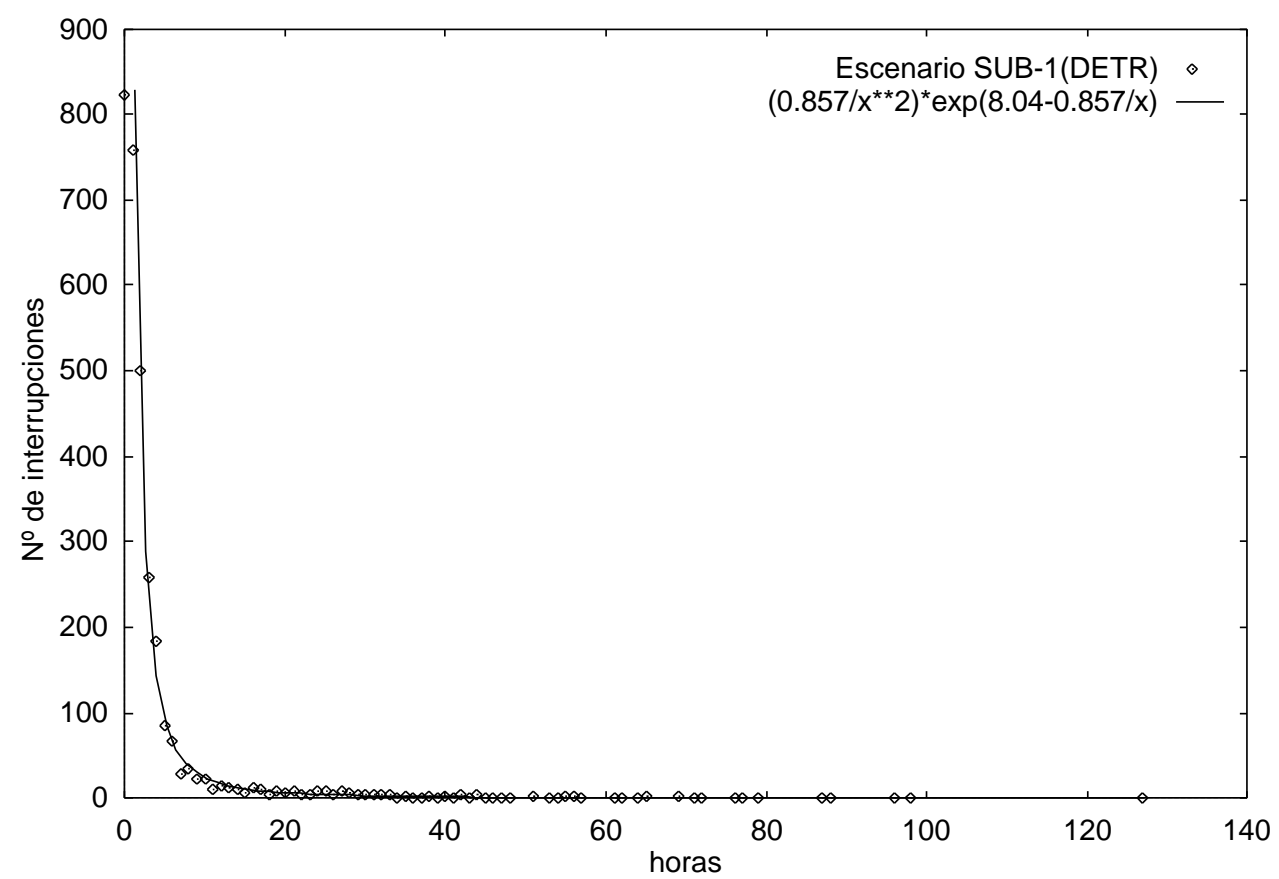

Fig.4.15.-Ajuste por una función del tipo exponencial de la distribución de tiempo de indisponibilidad para el punto de carga número " 0 " de la red subterránea en el escenario básico .

La parametrización de cada distribución aparece en la esquina superior derecha de cada figura. A partir de esta función, para hallar la probabilidad de que un suceso se produzca en un intervalo de tiempo dado, sólo tenemos que normalizar la expresión anterior e integrarla en ese intervalo de tiempo. En este caso, la probabilidad de que la duración de una interrupción sea menor que una de valor arbitrario $x$ vendrá dada por:

$$
p(x)=e^{-\frac{b}{x}}
$$

En la figura 4.16 se recogen los resultados de la simulación para la distribución del tiempo hasta la interrupción, del punto de carga número " 0 ", donde se puede apreciar que la mayoría de las interrupciones se producen antes de las 200.000 horas de funcionamiento ininterrumpido, aunque en alguna ocasión se lleguen a las 450.000 horas de funcionamiento ininterrumpido, que es el límite superior de continuidad de suministro en el punto de carga "0". El límite inferior, por debajo del cual se puede garantizar que no habrá interrupción de suministro se encuentra hacia las 2.300 horas.

La distribución del tiempo hasta la interrupción de la figura 4.16 puede ajustarse por una función exponencial del tipo:

$$
f(x)=e^{(a-b x)}
$$

como puede comprobarse en la figura 4.17.

La conclusión es general para cualquier punto de carga. 


\section{CAPÍTULO 4 - RESULTADOS}

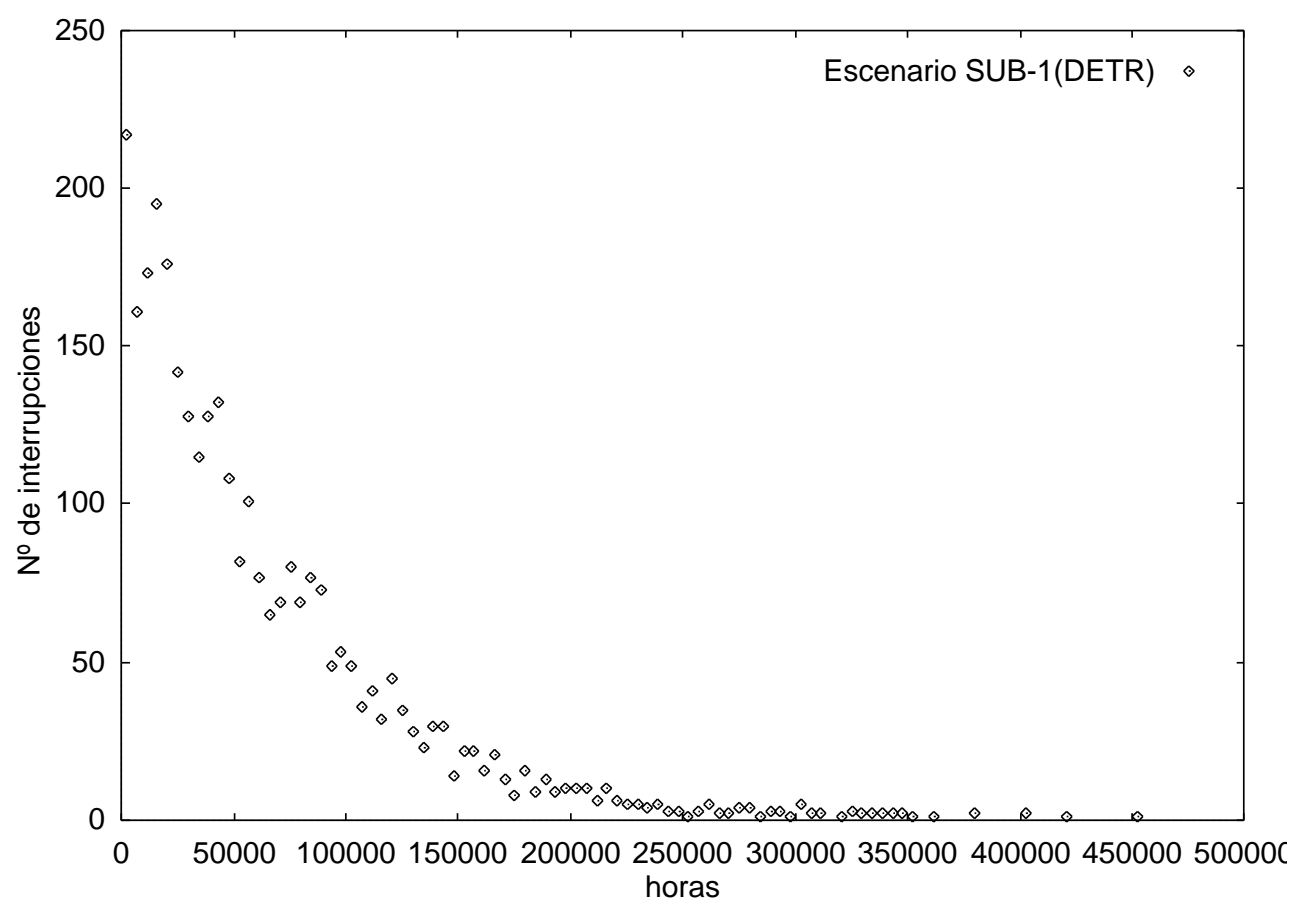

Fig.4.16.-Distribución de tiempo hasta la interrupción para el punto de carga número " 0 " de la red subterránea para el caso básico.

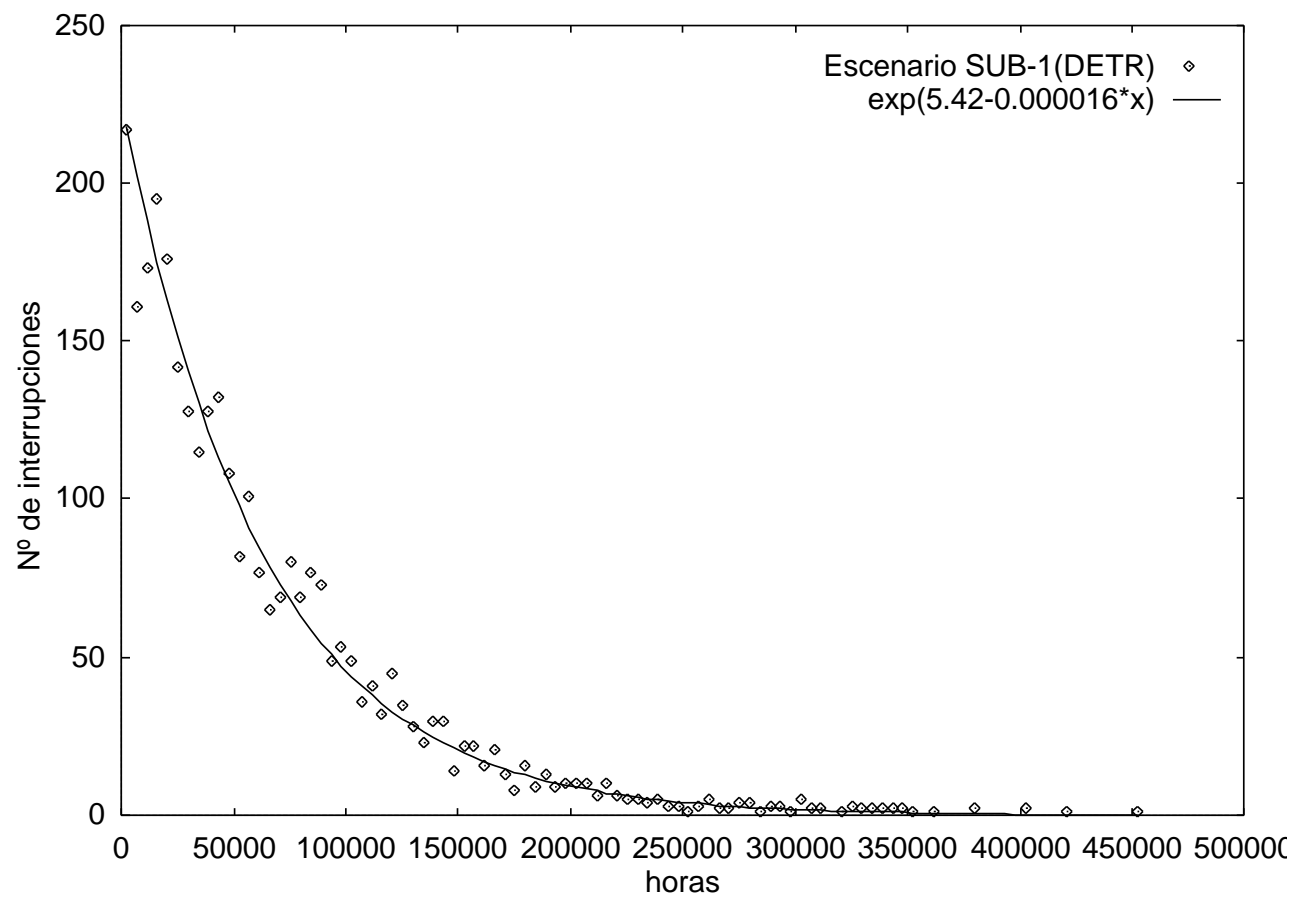

Fig.4.17.-Ajuste por una función exponencial de la distribución de tiempo hasta la interrupción para el punto de carga número " 0 " de la red subterránea en el escenario básico. 
Es igualmente general el hecho de que en la ley de distribución del tiempo hasta la interrupción de cada punto de carga, solamente influya el tipo de distribución del tiempo hasta el fallo de los elementos que forman la red, y ésta en definitiva, es exponencial para todos los escenarios. Por consiguiente, es indiferente que el Tiempo de Reparación obedezca a una Distribución Exponencial (DETR) o a una Normal Truncada (DNTR), pues el tiempo hasta la interrupción de cada punto de carga será sistemáticamente exponencial. No sucederá así con la ley de distribución del tiempo de indisponibilidad como veremos a continuación.

En consecuencia en todos los escenarios, para hallar la probabilidad de que se llegue a un tiempo arbitrario $x$ sin que el servicio se halla interrumpido sólo deberemos normalizar e integrar la función de densidad de probabilidad anterior entre $x$ e $\infty$, obteniendo la expresión:

$$
p(x)=e^{-b x}
$$

\section{Escenario SUB-2 (DETR)}

Si multiplicamos la tasa de fallo del cable subterráneo CS0 y el interruptor automático IA4, y examinamos la distribución del tiempo de indisponibilidad para el punto de carga número "10", el resultado es el de la figura 4.18.

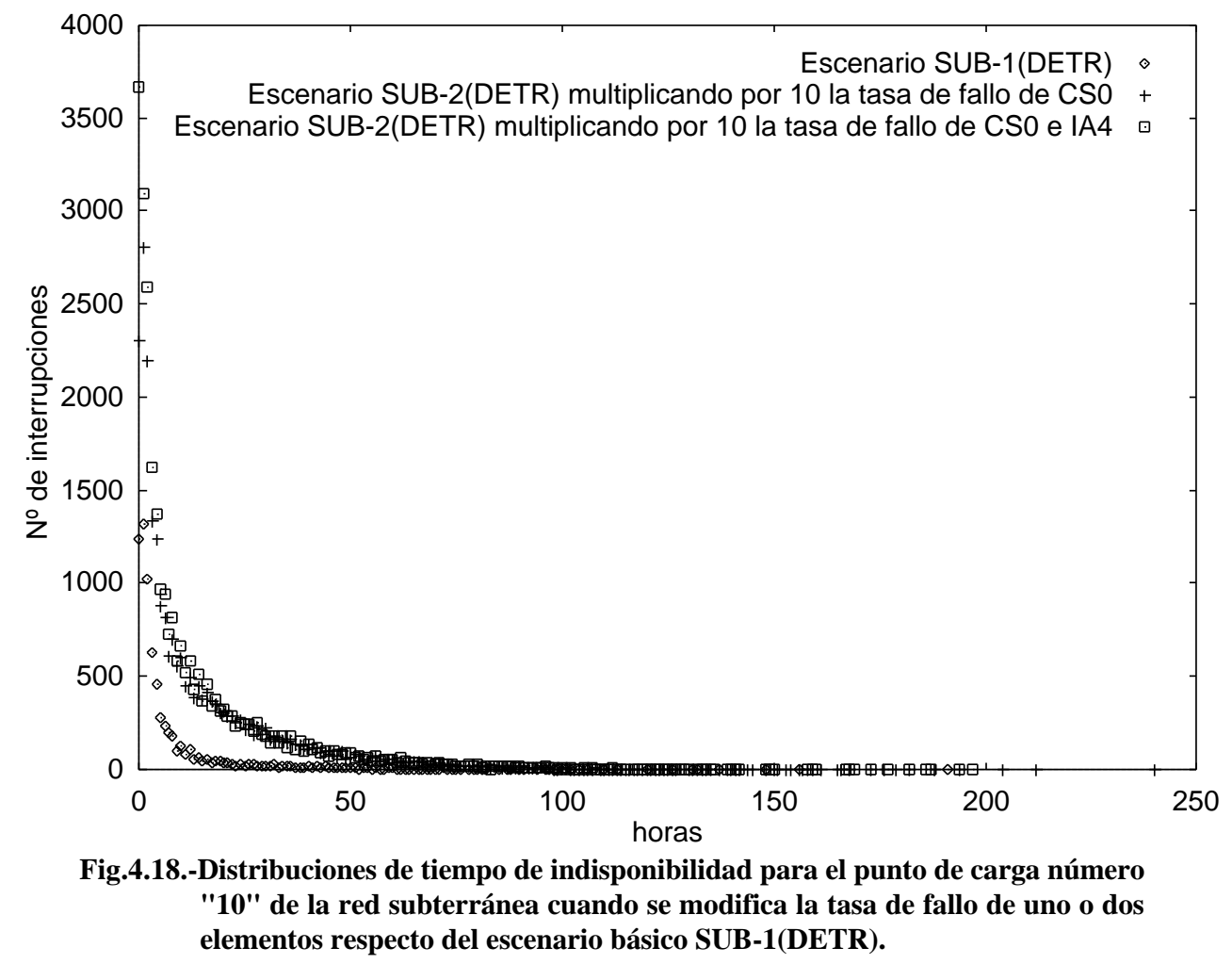


Se aprecia que, cuando el elemento CSO tiene una tasa de fallo 10 veces superior a la que tenía, lo que le sitúa con un valor muy por encima de la de los demás elementos, la ley de distribución del tiempo de indisponibilidad es la del escenario básico SUB-1. Las frecuencias de aparición de los valores alrededor del tiempo medio de reparación de CS0, 20 horas, crecen significativamente. Cuando, simultáneamente, se multiplican por 10 la tasa de fallo de CS0 e IA4 la ley de distribución es la misma que antes y las frecuencias de aparición de los valores alrededor del tiempo medio de reparación de CS0, esto es, alrededor de 20 horas, permanecen iguales, aumentando únicamente las frecuencias de aparición de los valores cercanos a la media de reparación de IA4, esto es, alrededor de las 3 horas.

El límite superior de tiempo de indisponibilidad permanece, básicamente, en el valor que poseía en el escenario básico, o sea 200 horas.

Las distribuciones del tiempo de indisponibilidad de la figura 4.18 correspondientes al escenario SUB-2 (DETR) pueden ajustarse por una distribución suma de dos exponenciales:

$$
f(x)=e^{(a-b x)}+e^{\left(a^{\prime}-b^{\prime} x\right)}
$$

como puede comprobarse en la figura 4.19.

La conclusión es general para cualquier punto de carga.

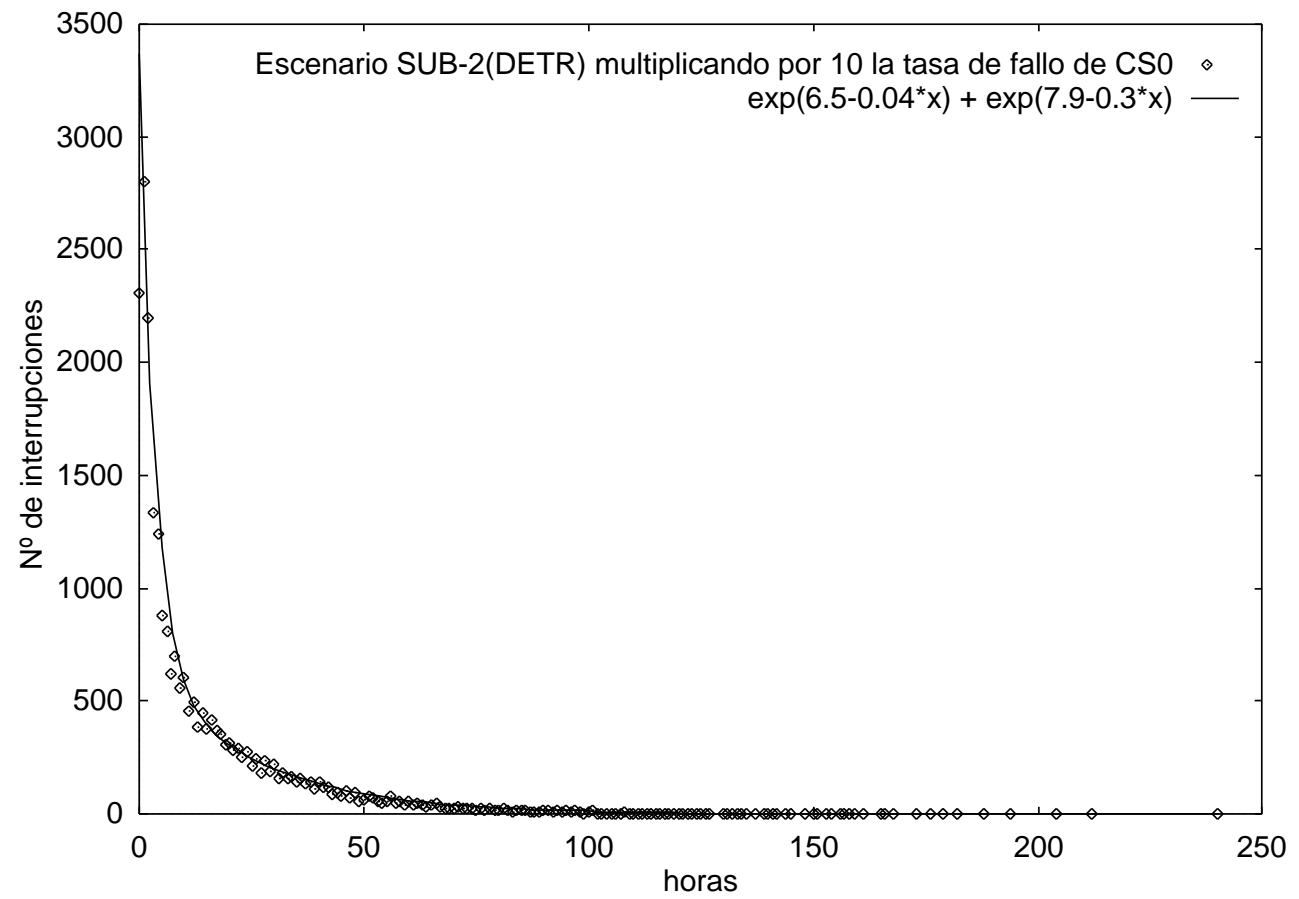

Fig.4.19.-Ajuste por una suma de dos exponenciales de la distribución del tiempo de indisponibilidad para el punto de carga número "10" de la red subterránea cuando se multiplica por 10 la tasa de fallo de CSO. 
Si multiplicamos por 10 la tasa de fallo del elemento CS0 aumenta espectacularmente el número de interrupciones totales, tardando éstas menos en producirse, véase figura 4.20. Se puede observar cómo disminuye el límite superior de continuidad de suministro, pasando de 298.000 horas en el escenario SUB-1 (DETR) a 78.000 horas en el escenario SUB-2 (DETR). También el límite inferior de continuidad de suministro, pasa de 1.500 horas en el escenario SUB-1 (DETR) a 390 horas en el escenario SUB-2 (DETR).

Si se multiplica por 10 la tasa de fallo de CS0 e IA4, simultáneamente, la distribución de tiempo hasta la interrupción es muy parecida a la anterior, pues la influencia de IA4 es muy pequeña frente a CSO, ya que la tasa de fallo de CS0 es muy superior a la de IA4. Además CS0 es más numeroso en la red, razones por las que resulta más influyente en la indisponibilidad de la red que IA4. La única diferencia perceptible con la anterior distribución la constituye el aumento en la frecuencia de aparición de duraciones de continuidad de suministro inferiores a las 12.000 horas (que son las que corresponden al fallo de IA4). El límite superior e inferior de continuidad de suministro permanecen, básicamente, en los valores de 78.000 y 390 horas, respectivamente (ver figura 4.20).

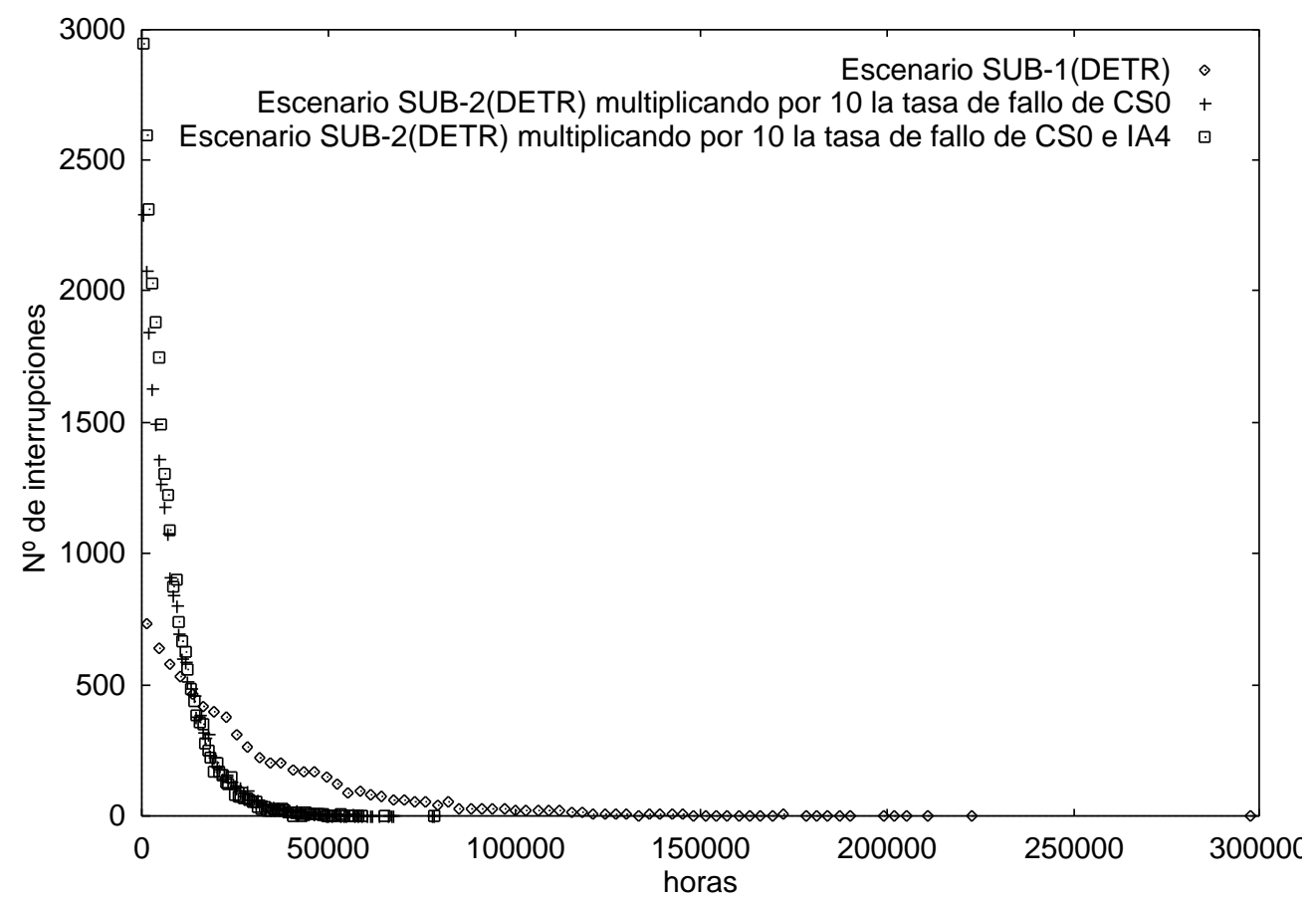

Fig.4.20.-Distribuciones de tiempo hasta la interrupción, para el punto de carga número "10" de la red subterránea cuando se modifica la tasa de fallo de uno o dos elementos respecto el escenario básico SUB-1(DETR). 


\section{Escenario SUB-3 (DETR)}

En este escenario, como en el precedente, la ley de distribución del tiempo hasta la interrupción de los puntos de carga no varía respecto a la del caso básico, pues, como ya se indicó, sólo modificamos los tiempos medios de reparación de uno o dos elementos que condicionan, únicamente, las distribuciones del tiempo de indisponibilidad de los puntos de carga.

En las figuras 4.21 y 4.22 se muestra lo que sucede cuando multiplicamos por 5 el tiempo de reparación de los elementos CS0 e IA4, ya escogidos en el escenario anterior.

En la figura 4.21 se aprecia que, si el elemento CS0 (el más numeroso) tuviese un tiempo medio de reparación cinco veces mayor del inicial, quedando con un tiempo de reparación muy superior al del resto de los elementos (20 veces superior al de cualquier otro), aumenta el límite superior de tiempos de indisponibilidad,desde 170 horas (en el escenario básico) a 630 horas.

Si también multiplicamos por 10 el tiempo de reparación del elemento IA4, el resultado, figura 4.22, es prácticamente el mismo que en el caso anterior, ya que este elemento no es tan numeroso como el CS0 y tiene una tasa de fallo 4 veces menor. $\mathrm{Su}$ influencia, por tanto, es menor en el número de interrupciones totales, concluyendo de forma lógica, que sólo la modificación de los tiempos de reparación de los elementos que fallan más a menudo influye apreciablemente en la distribución de tiempos de indisponibilidad.
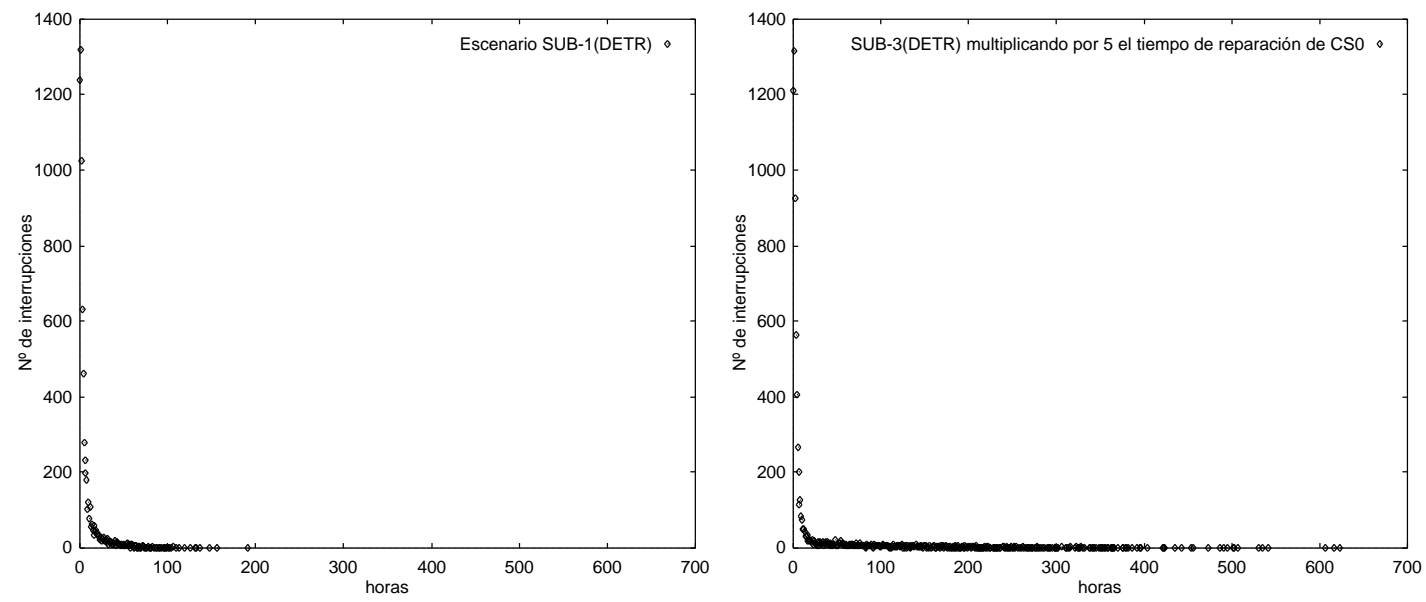

Fig.4.21.-Distribuciones de tiempo de indisponibilidad para el punto de carga número "10" de la red subterránea en el escenario básico, a la izquierda, y en el escenario SUB-3 (DETR) cuando se multiplica por 5 el tiempo de reparación de CSO. 

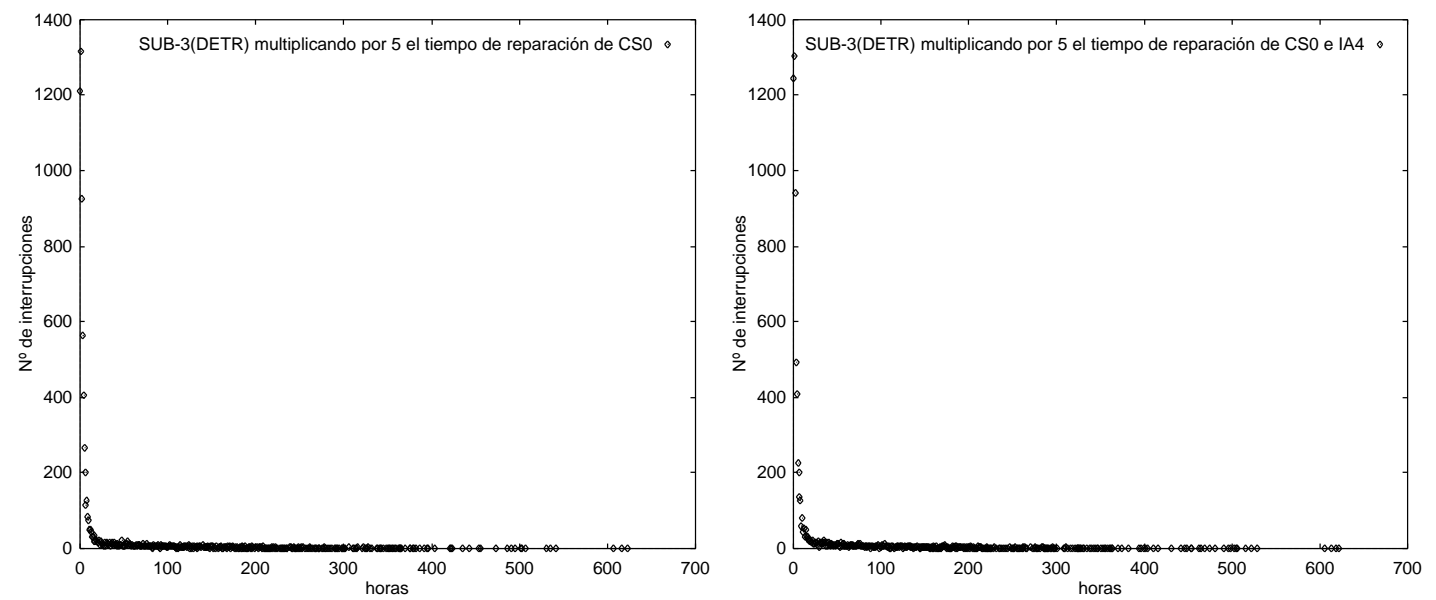

Fig.4.22.-Distribuciones de tiempo de indisponibilidad para el punto de carga número "10" de la red subterránea en el escenario SUB-3 (DETR) cuando se multiplica por 5 el tiempo de reparación de CSO y de IA4.

Las distribuciones del tiempo de indisponibilidad de las figuras 4.21 y 4.22 correspondientes al escenario SUB-3 (DETR) pueden ajustarse por una distribución suma de dos exponenciales:

$$
f(x)=e^{(a-b x)}+e^{\left(a^{\prime}-b^{\prime} x\right)}
$$

como puede comprobarse en la figura 4.23.

La conclusión es general para cualquier punto de carga.

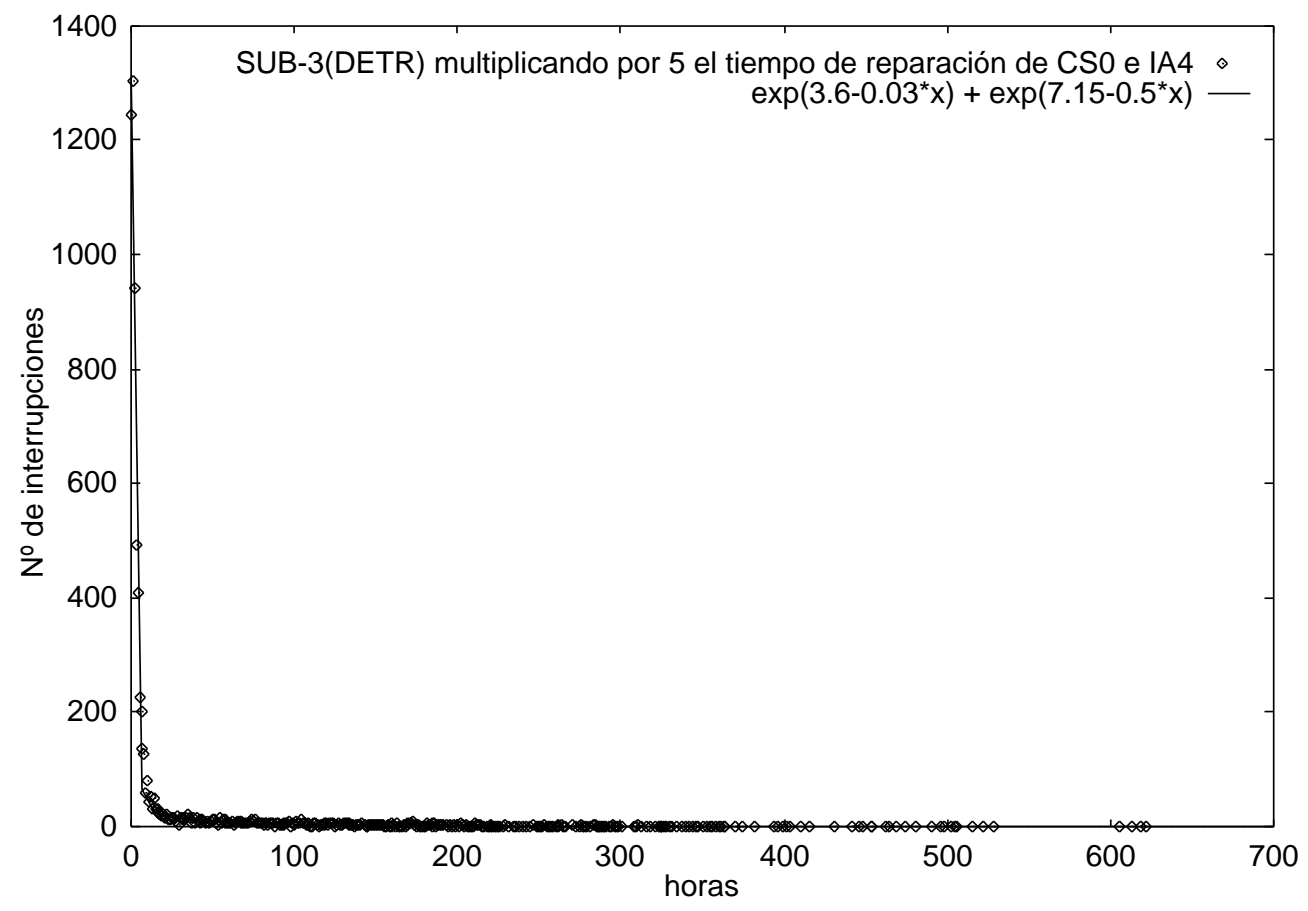

Fig.4.23.-Ajuste por una suma de dos exponenciales de la distribución de tiempo de indisponibilidad para el punto de carga número "10" de la red subterránea cuando se multiplica por 5 el tiempo de reparación de CSO e IA4. 


\section{Escenario SUB-4 (DETR)}

En las figuras 4.24 y 4.25 se muestran los resultados de la simulación, cuando incrementamos, simultánea y conjuntamente, la tasa de fallo y el tiempo de reparación de CS0 e IA4. Multiplicaremos sus tasas de fallo por 10 y sus tiempos medios de reparación por 5.

Como se aprecia en la figura 4.24, al igual que sucedía en el escenario SUB-2 (DETR), el número de interrupciones provocados por el elemento CS0 son, ahora, muy superiores a los provocados por el resto de los elementos, incrementando las frecuencias de aparición de las duraciones de indisponibilidad cercanas al valor medio de 100 horas, que corresponde al tiempo de reparación de CSO. También se puede apreciar, que el límite superior del tiempo de indisponibilidad es alrededor de 4 veces mayor que el obtenido cuando únicamente cambiábamos la tasa de fallo de CS0 en el escenario SUB-2 (DETR), debido al incremento en el tiempo de reparación de CSO.

Si, además, también multiplicamos por 10 y por 5 , la tasa de fallo y el tiempo de reparación, respectivamente, del elemento IA4, véase figura 4.25, la distribución del tiempo de indisponibilidad resulta ser muy parecida a la anterior, como ya nos sucedió en el escenario SUB-2 (DETR), pues CS0 resulta más influyente en la indisponibilidad de la red que IA4. La única diferencia perceptible con la distribución de la figura 4.23 la constituye el aumento en la frecuencia de aparición de las interrupciones de duración cercana a las 16,5 horas (que son las que corresponden al fallo de IA4).

No se han representado las distribuciones de tiempo hasta la interrupción en este escenario, pues son iguales a las del escenario SUB-2 (DETR). La única diferencia respecto al escenario SUB-2 (DETR) es que, además de modificar la tasa de fallo de CS0 e IA4, ahora también modificamos sus tiempos medios de reparación, lo que, ciertamente, no influye en el número de interrupciones que se producen en la red.

La distribución del tiempo de indisponibilidad de la figura 4.24 correspondiente al escenario SUB-3 (DETR) también puede ajustarse por una distribución suma de dos exponenciales como ocurría en el escenario SUB-2 (DETR). Como puede comprobarse en la figura 4.25.

La conclusión es general para cualquier punto de carga. 


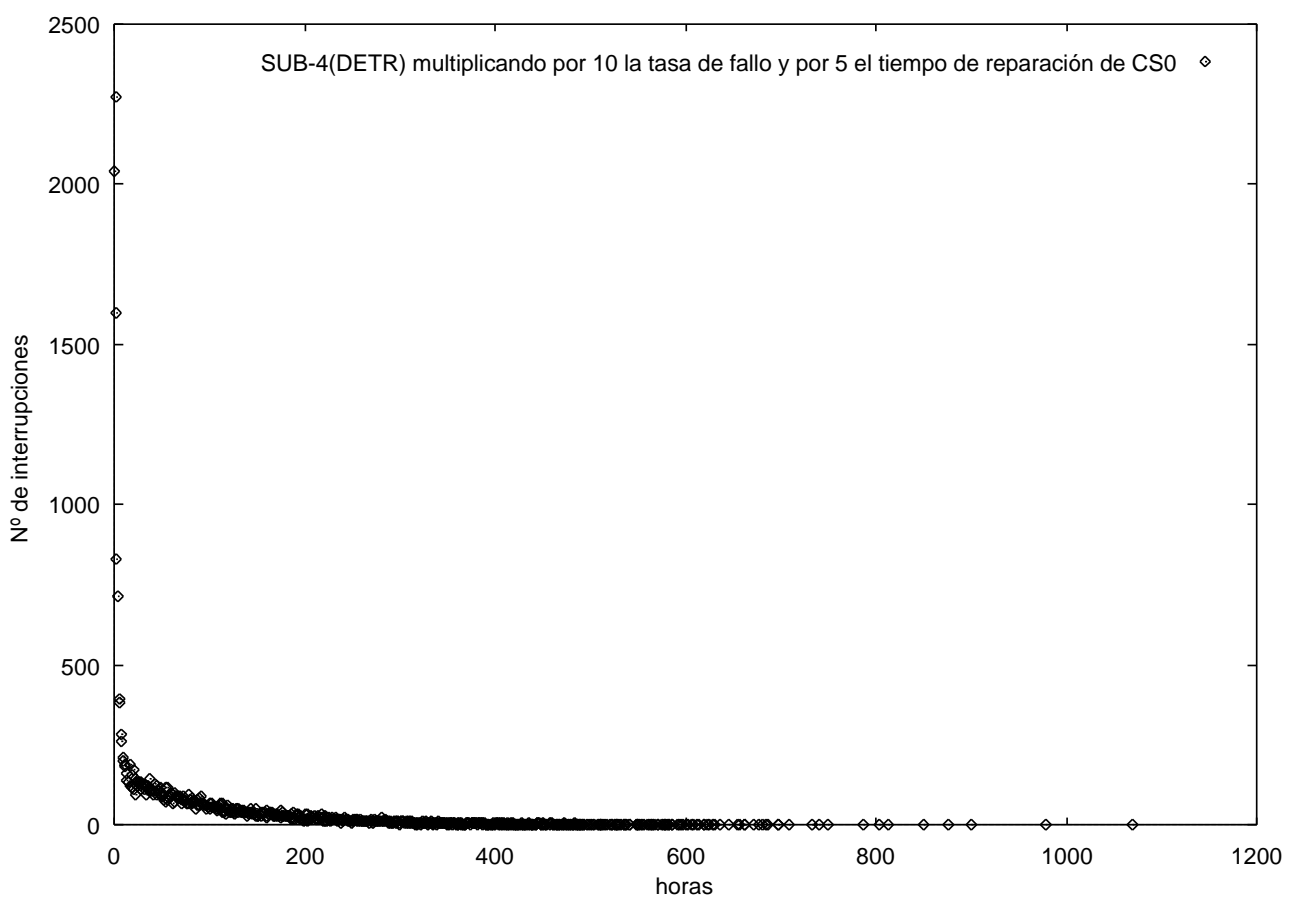

Fig.4.24.-Distribución del tiempo de indisponibilidad para el punto de carga número "10" de la red subterránea cuando se modifica, conjuntamente, la tasa de fallo y el tiempo de reparación de CSO.

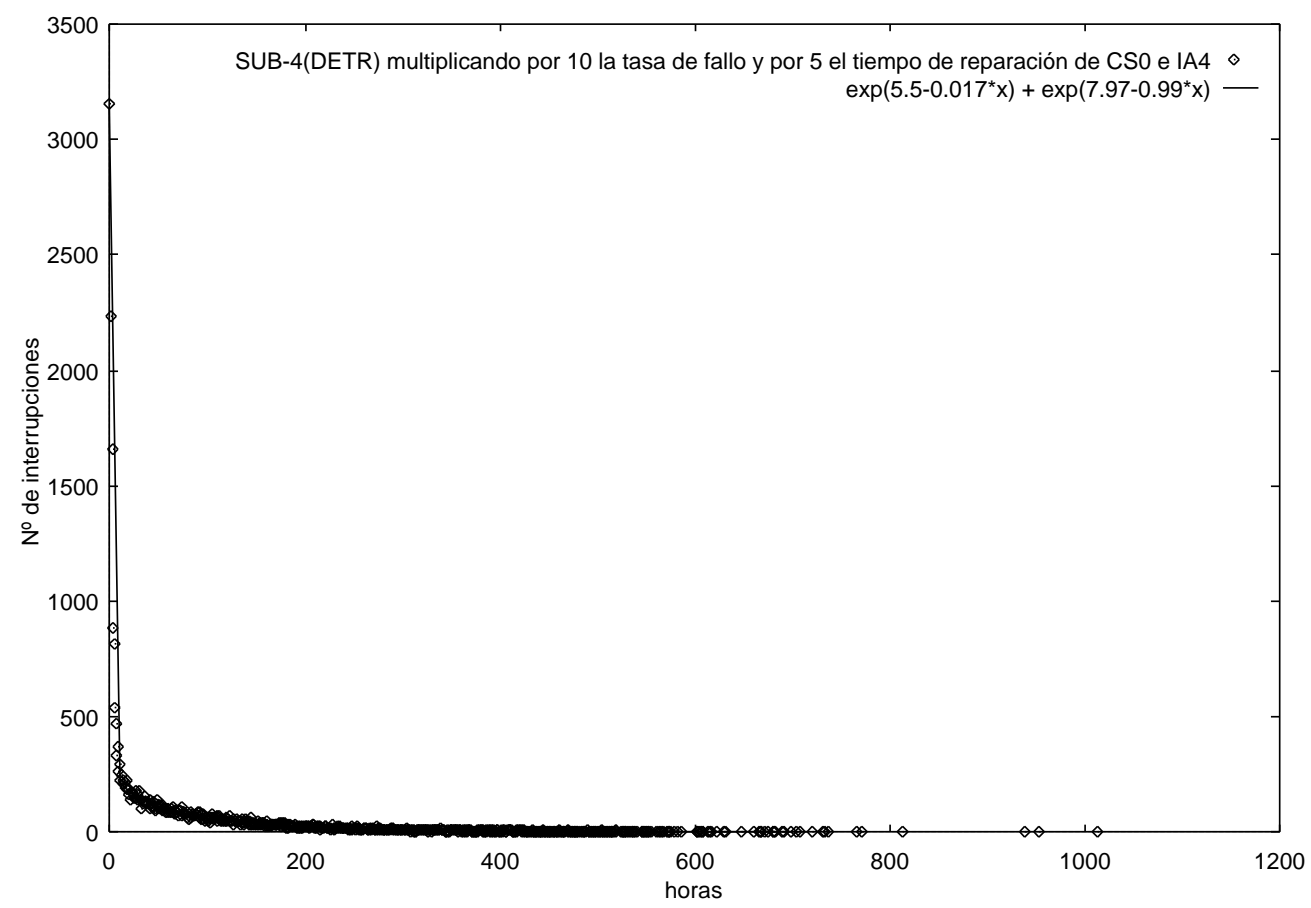

Fig.4.25-Ajuste por una suma de dos exponenciales de la distribución de tiempo de indisponibilidad para el punto de carga número "10" de la red subterránea cuando se modifica, conjuntamente, la tasa de fallo y el tiempo de reparación de CSO e IA4.

DISTRIBUCIÓN NORMAL PARA EL TIEMPO DE REPARACIÓN (DNTR) DE LOS ELEMENTOS DE LA RED. 
Realizaremos, ahora, un análisis similar al ya efectuado, pero considerando como hipótesis de distribución de los tiempos de reparación de los elementos de la red una distribución normal truncada. Los valores para las tasas de fallo y tiempos de reparación de los elementos en los distintos escenarios será el mismo.

Como la distribución elegida para el tiempo hasta el fallo de los elementos de la red sigue siendo la exponencial y los valores para sus tasas de fallo continúan siendo los mismos, las distribuciones del tiempo hasta la interrupción de cada punto carga serán las mismas que en los escenarios precedentes. De ahí que, en este apartado, sólo nos detengamos en el estudio de las distribuciones del tiempo de indisponibilidad de los puntos carga.

\section{Escenario SUB-1 (DNTR)}

En la figura 4.26, aunque se aprecia cierta semejanza entre la distribución del tiempo de indisponibilidad de nuestro escenario SUB-1 (DNTR) y la del escenario básico SUB-1 (DETR), podemos distinguir tres máximos en la distribución SUB-1 (DNTR) que no aparecían en la SUB-1 (DETR), en que la ley de distribución se comportaba de manera uniformemente decreciente. Uno de los máximos corresponde a la suma de los tiempos de localización y maniobra de cualquier elemento de la red, 2 horas aproximadamente; el segundo máximo, que apenas se percibe, centrado en las 4.5 horas, corresponde a la suma de los tiempos de localización y reparación del elemento IA4; el tercer y último máximo, centrado en las 21.5 horas, corresponde a la suma de los tiempos de localización y reparación del elemento CS0, este máximo es el que más resalta de los tres ya que la tasa de fallo del elemento CSO es superior a la del resto de los elementos.

También podemos apreciar que las frecuencias de aparición en la distribución de este escenario SUB-1 (DNTR) presenta una dispersión menor que su equivalente con tiempos de reparación exponenciales SUB-1 (DETR), provocando que el límite superior de la duración de la interrupción disminuya de las 191 horas a las 42 horas. La causa de esta menor dispersión es el truncamiento de las colas de las normales que empleamos como ley de distribución para el tiempo de reparación de los elementos.

La distribución del tiempo de indisponibilidad de la figura 4.26 correspondiente al escenario SUB-1 (DNTR), puede ajustarse mediante la suma de tres distribuciones normales, como puede comprobarse en la figura 4.27.

$$
f(x)=\frac{1}{\sigma_{0} \sqrt{2 \pi}} \cdot e^{-\frac{\left(x-\mu_{0}\right)^{2}}{2 \sigma_{0}^{2}}}+\frac{1}{\sigma_{1} \sqrt{2 \pi}} \cdot e^{-\frac{\left(x-\mu_{1}\right)^{2}}{2 \sigma_{1}^{2}}}+\frac{1}{\sigma_{2} \sqrt{2 \pi}} \cdot e^{-\frac{\left(x-\mu_{2}\right)^{2}}{2 \sigma_{2}^{2}}}
$$

Esta conclusión es general para cualquier punto de carga. 


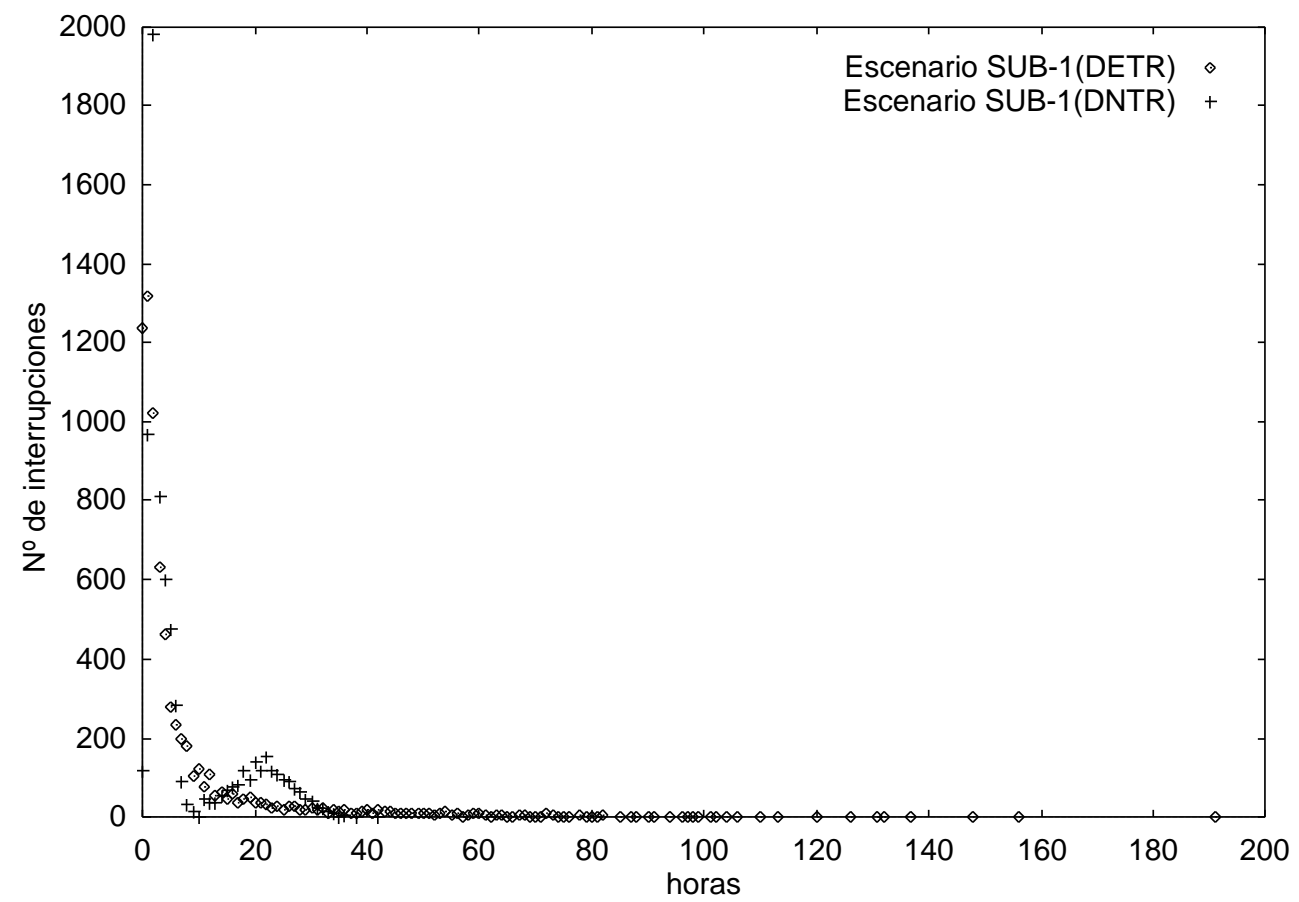

Fig.4.26.-Comparación entre las distribuciones del tiempo de indisponibilidad para el punto de carga número " 10 " de la red subterránea en el supuesto de que el tiempo de reparación de los elementos se considere distribuido exponencialmente o normalmente.

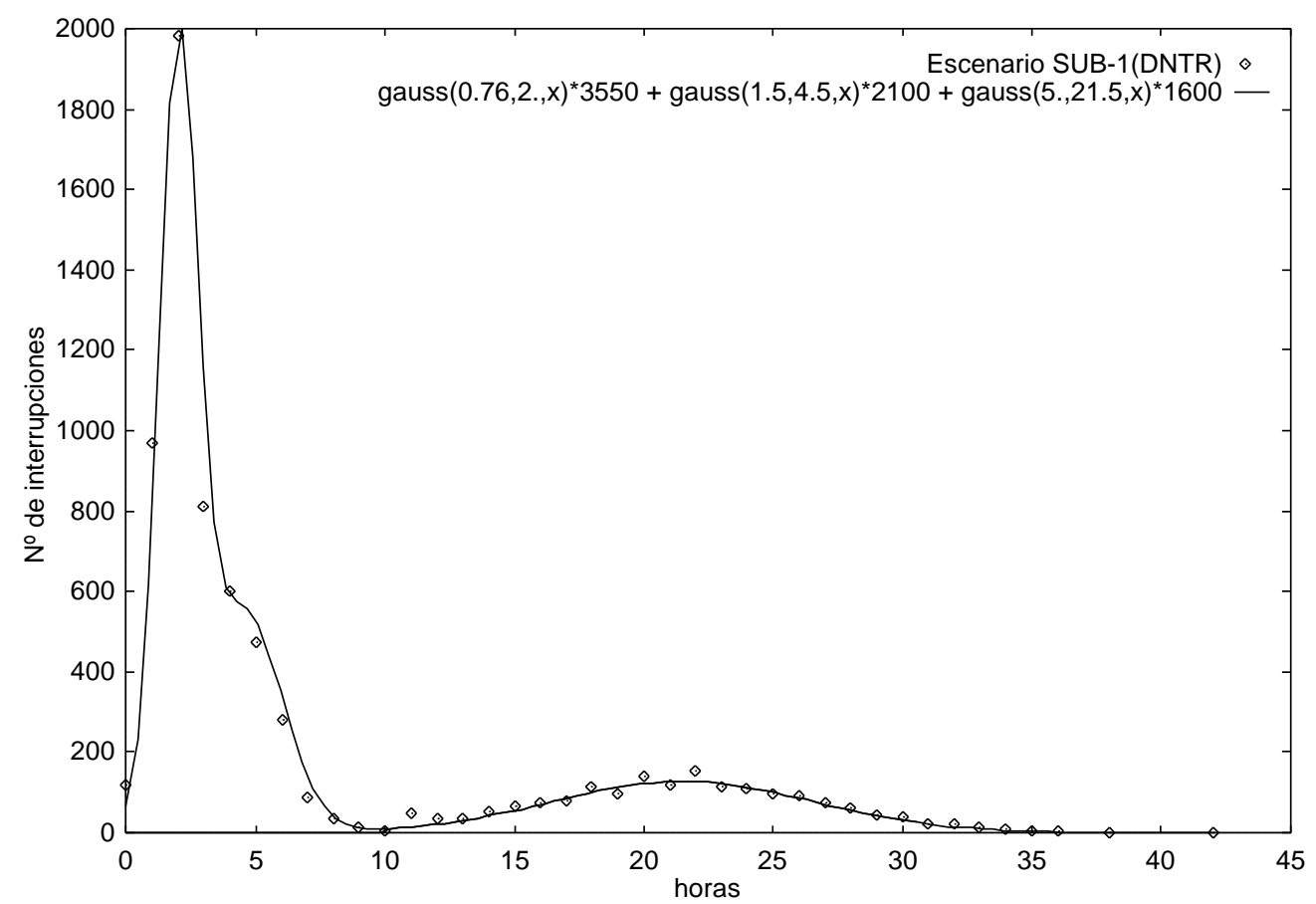

Fig.4.27.-Ajuste por una suma de tres funciones normales de la distribución del tiempo de indisponibilidad del punto de carga número "10" de la red subterránea para el escenario SUB-1 (DNTR).

\section{Escenario SUB-2 (DNTR)}


Si multiplicamos por 10 la tasa de fallo que CS0 poseía en el escenario precedente SUB-1 (DNTR), se aprecia, en la figura 4.28, que la distribución de tiempos de indisponibilidad experimenta dos incrementos notables en las frecuencias de aparición correspondientes a los dos máximos que presenta, en las 2 y en las 21,5 horas. Esto es debido a que, ahora, el aumento de la tasa de fallo de CS0 provocará más interrupciones de suministro, debiéndose reparar y maniobrar muchas más veces que antes.

$\mathrm{Si}$, además, multiplicamos por 10 la tasa de fallo al elemento IA4, se aprecia un incremento en la frecuencia de aparición del máximo que se encuentra sobre el valor correspondiente a la suma del tiempo de reparación de IA4 más su tiempo de localización, 4,5 horas.

En la figura 4.29, comparamos las distribuciones del tiempo de indisponibilidad para el punto de carga número "10" en este escenario y en el escenario SUB-2 (DETR) en el que el tiempo de reparación de los elementos se consideraba distribuido exponencialmente. Como en el escenario anterior, se aprecia la pérdida del carácter uniformemente decreciente que poseía la distribución del tiempo de indisponibilidad en el escenario SUB-2 (DETR), surgiendo tres máximos en la distribución SUB-1 (DNTR).

También podemos apreciar que las frecuencias de aparición en la distribución de este escenario SUB-2 (DNTR) presenta una dispersión menor que su equivalente con tiempos de reparación exponenciales SUB-2 (DETR), provocando que el límite superior de la duración de la interrupción disminuya de las 240 horas a las 52 horas.

La distribución del tiempo de indisponibilidad de la figura 4.28 correspondiente al escenario SUB-2 (DNTR) cuando multiplicamos por 10 la tasa de fallo de CSO e IA4, puede ajustarse mediante la suma de cuatro distribuciones normales

$$
f(x)=\frac{1}{\sigma_{0} \sqrt{2 \pi}} \cdot e^{-\frac{\left(x-\mu_{0}\right)^{2}}{2 \sigma_{0}^{2}}}+\frac{1}{\sigma_{1} \sqrt{2 \pi}} \cdot e^{-\frac{\left(x-\mu_{1}\right)^{2}}{2 \sigma_{1}^{2}}}+\frac{1}{\sigma_{2} \sqrt{2 \pi}} \cdot e^{-\frac{\left(x-\mu_{2}\right)^{2}}{2 \sigma_{2}^{2}}}+\frac{1}{\sigma_{3} \sqrt{2 \pi}} \cdot e^{-\frac{\left(x-\mu_{3}\right)^{2}}{2 \sigma_{3}^{2}}}
$$

como puede comprobarse en la figura 4.30 .

La conclusión es general para cualquier punto de carga. 


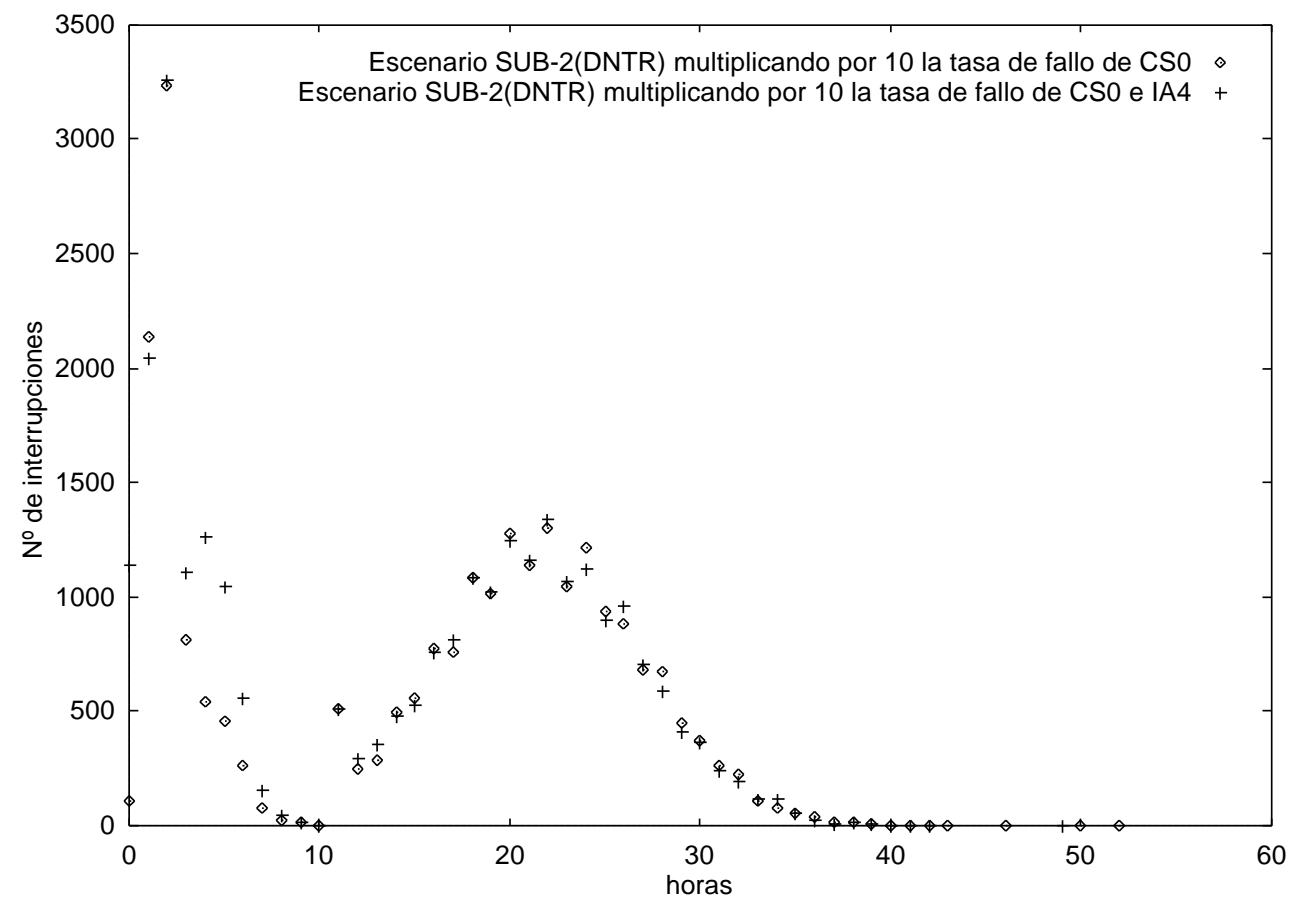

Fig.4.28.-Comparación entre las distribuciones del tiempo de indisponibilidad para el punto de carga número "10" de la red subterránea cuando se modifica la tasa de fallo de uno o dos elementos.

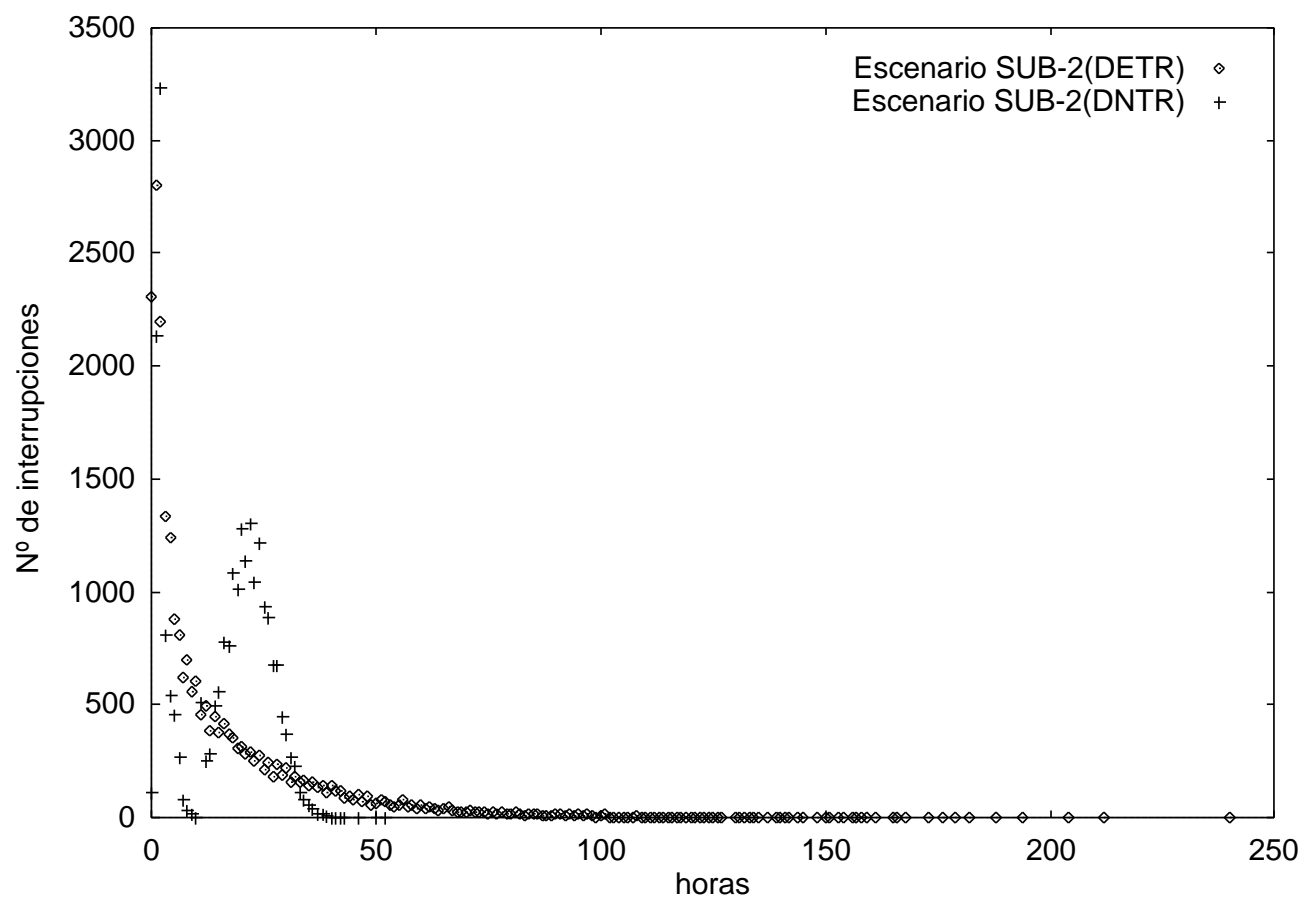

Fig.4.29.-Comparación entre las distribuciones del tiempo de indisponibilidad para el punto de carga número "10" de la red subterránea en el supuesto de que el tiempo de reparación de los elementos se considere distribuido exponencialmente o normalmente. 


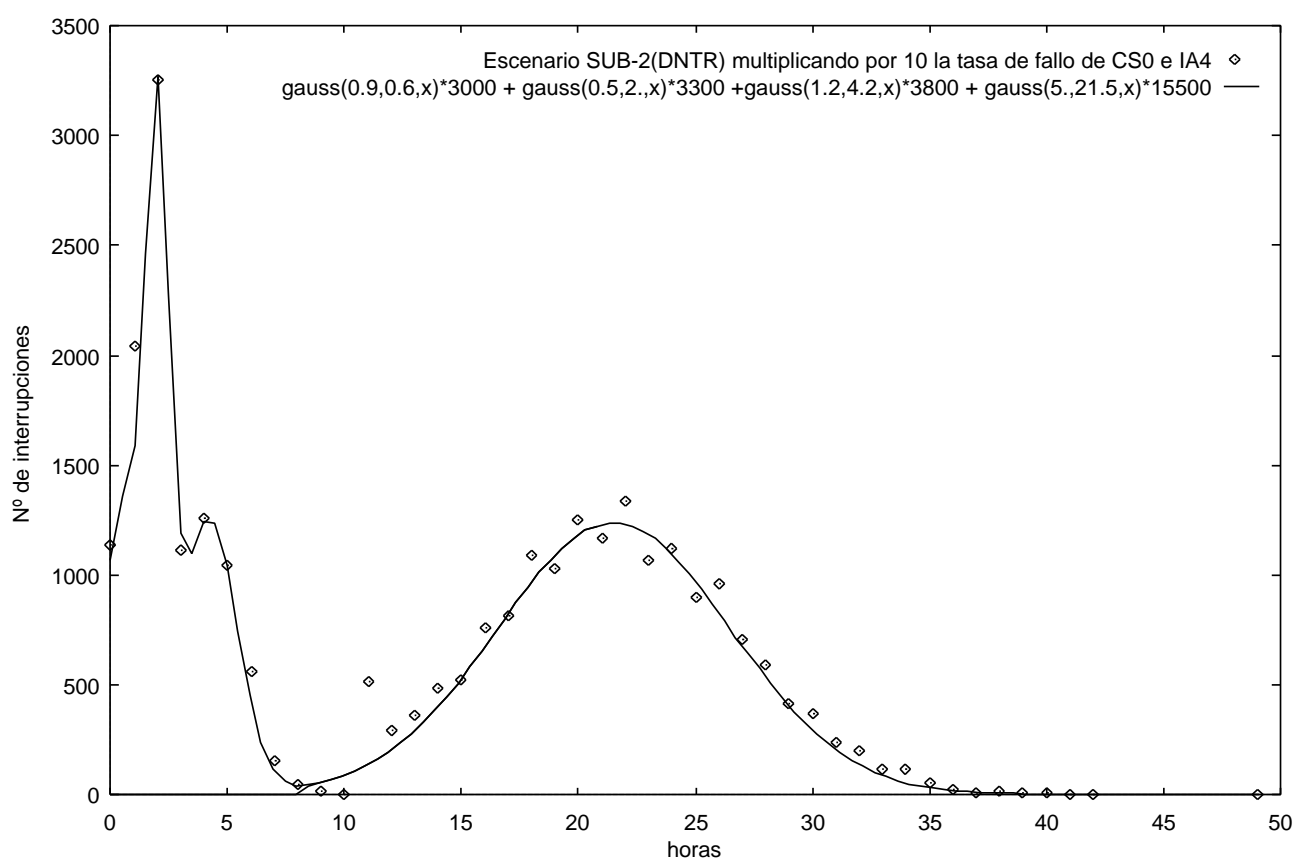

Fig.4.30.-Ajuste por una suma de cuatro funciones normales de la distribución del tiempo de indisponibilidad del punto de carga número "10" cuando multiplicamos por 10 la tasa de fallo de CSO e IA4.

\section{Escenario SUB-3 (DNTR)}

En la figura 4.31 se muestra cómo, cuando CS0 tiene un tiempo de reparación 5 veces superior al que tenía, el máximo, que la anterior distribución de tiempos de indisponibilidad (figura 4.28) se presentaba en las 22 horas, se traslada hacia un valor resultado de la suma del nuevo tiempo de reparación de CSO más su tiempo de localización, 102 horas. También se aprecia que el límite superior del tiempo de indisponibilidad multiplica por cinco, aproximadamente, su valor.

Si además de a CS0, multiplicamos también por 5 el tiempo de reparación de IA4, observamos como el máximo existente en las 4,5 horas se traslada hacia un valor resultado de la suma del nuevo tiempo de reparación de IA4 más su tiempo de localización, 17 horas.

En la figura 4.32, comparamos los resultados de este escenario con los obtenidos para el escenario SUB-3 (DETR). Se aprecia el mismo comportamiento que en los escenarios anteriores, aunque en esta ocasión, los tres máximos de la distribución SUB-1 (DNTR) son menores que en los casos anteriores. Al igual que en los escenarios anteriores, el límite superior de la duración de la interrupción disminuye, en este caso, de 876 horas a 186 horas.

El ajuste de la distribución del tiempo de indisponibilidad de los puntos de carga en este escenario se realiza de forma similar a la del escenario SUB-2 (DNTR), mediante la suma de distribuciones normales. 


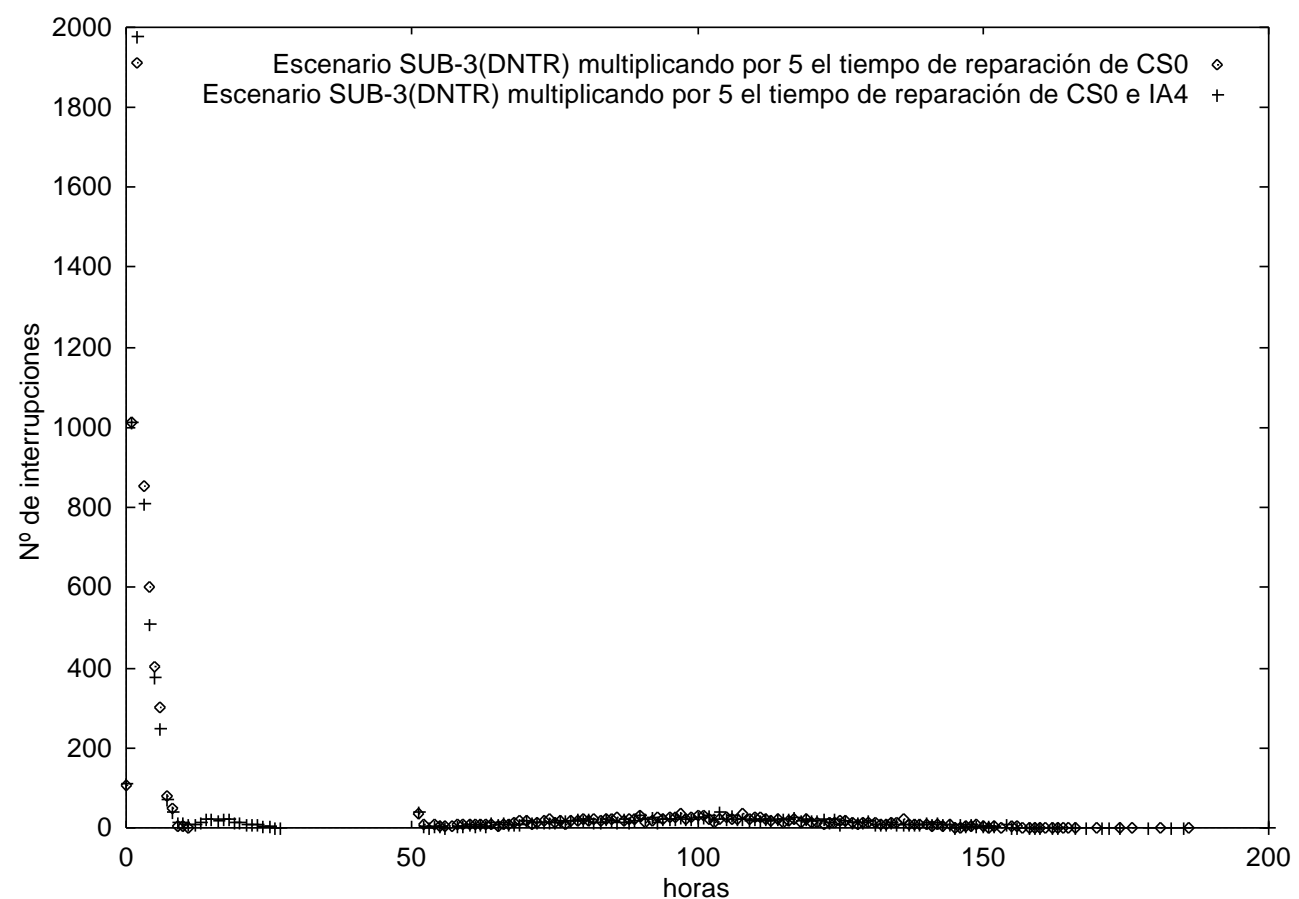

Fig.4.31.-Comparación entre las distribuciones del tiempo de indisponibilidad para el punto de carga número " $10 "$ de la red subterránea cuando se modifica el tiempo de reparación de algún elemento.

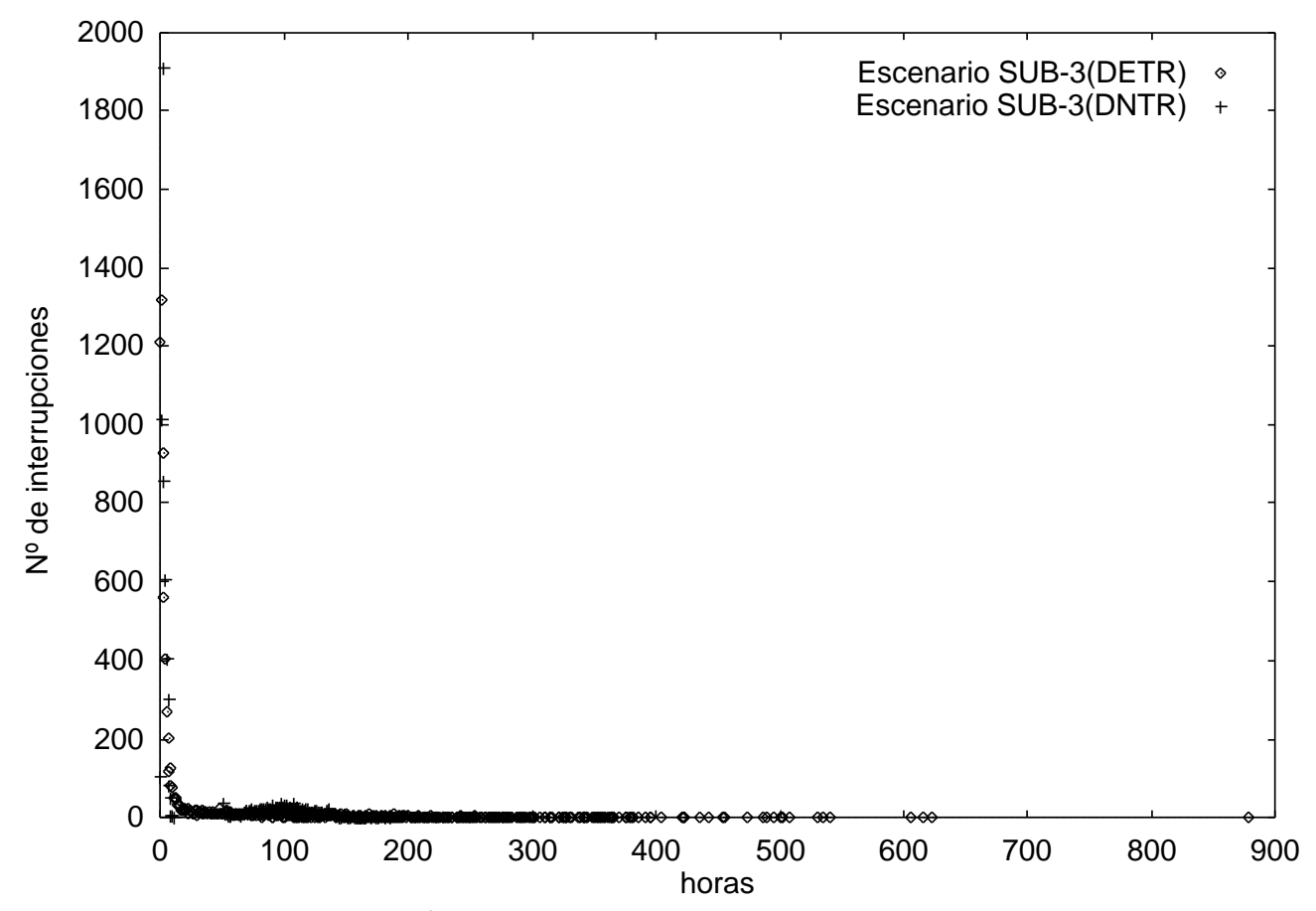

Fig.4.32.-Comparación entre las distribuciones del tiempo de indisponibilidad para el punto de carga número "10" de la red subterránea en el supuesto de que el tiempo de reparación de los elementos se considere distribuido exponencialmente o normalmente. 


\section{Escenario SUB-4 (DNTR)}

En este escenario, primeramente, multiplicamos, conjuntamente, por 10 la tasa de fallo y por 5 el tiempo de reparación que el elemento CS0 poseía en el escenario SUB-1 (DNTR). En la figura 4.33 se aprecia cómo la distribución de tiempos de indisponibilidad presenta tres máximos en los mismos valores que ya presentaba en el escenario SUB-3 (DNTR), en el que únicamente incrementamos el tiempo de reparación de CS0. Las frecuencias de aparición de los máximos de nuestro escenario crecen comparadas con las de SUB-3 (DNTR), de forma similar a como lo hacían los máximos del escenario SUB-2 (DNTR) comparados con SUB-1 (DNTR). Resultando el número de total de interrupciones del escenario SUB-4 (DNTR) igual al del escenario SUB-2 (DNTR).

Si además de al elemento CS0, también a IA4, le multiplicamos por 10 su tasa de fallo y por 5 su tiempo de reparación, todo lo dicho en comparación con los escenarios SUB-2 (DNTR) y SUB-3 (DNTR) es también aquí de aplicación.

La comparación entre los escenarios SUB-4 (DNTR) y SUB-4 (DETR), proporciona resultados similares a los encontrados para la comparación entre SUB-2 (DNTR) y SUB-2 (DETR).

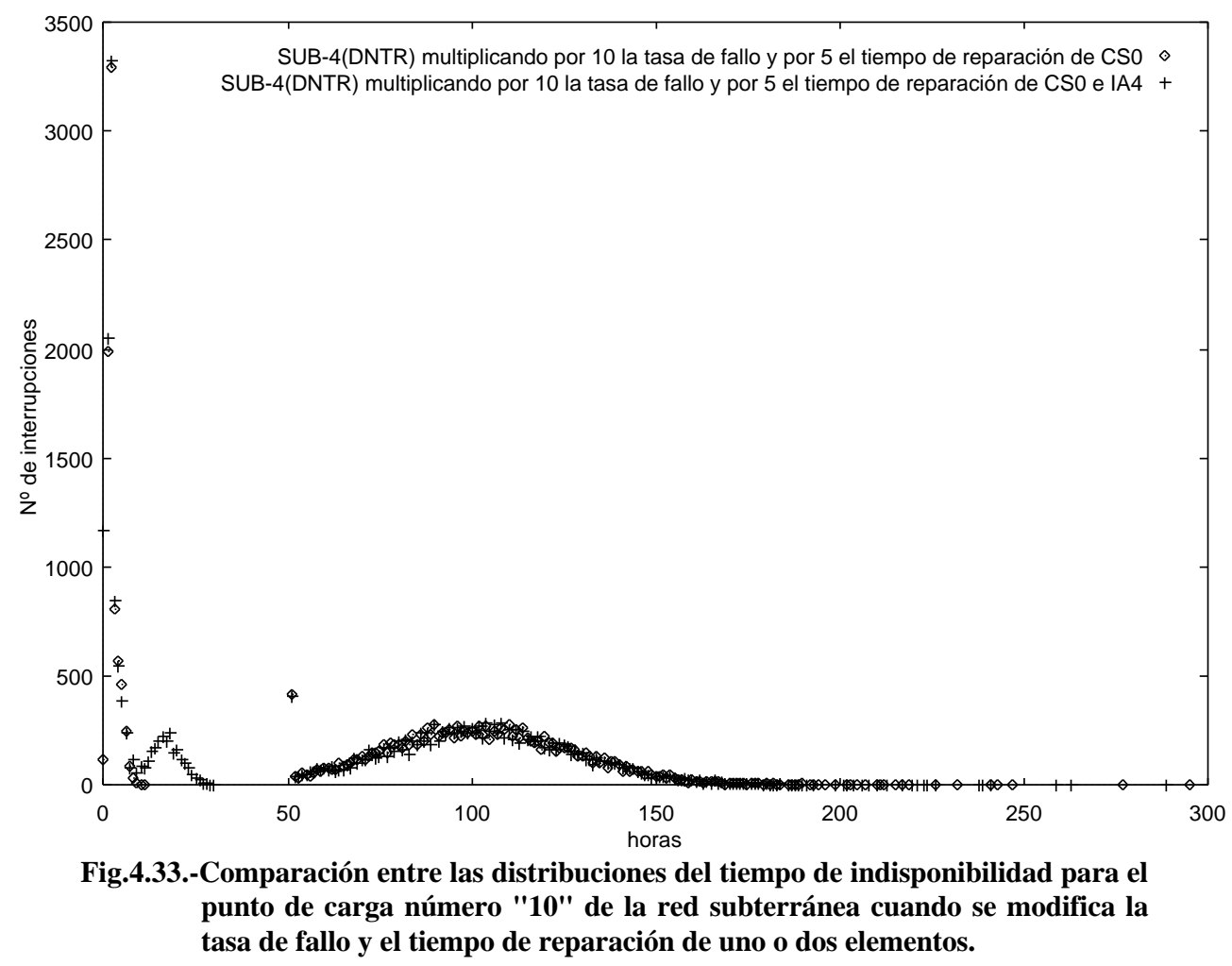

\section{ASPECTOS A DESTACAR SOBRE LAS REDES SUBTERRÁNEAS}


Hemos obtenido las distribuciones del tiempo hasta la interrupción y del tiempo de indisponibilidad en todos los escenarios analizados.

Hemos podido conocer los límites superior e inferior de las distribuciones del tiempo hasta la interrupción de los puntos de carga de la red en todos los escenarios analizados, constatando su estrecha dependencia con las tasas de fallo de los elemento que componen la red.

También medimos el límite superior de las distribuciones del tiempo de indisponibilidad y quedó patente su dependencia con los tiempos medios de reparación de los elementos y el tipo de distribución elegida para ésta. Registramos reducciones en un factor de 5, aproximadamente, al cambiar la distribución del tiempo medio de repación de los elementos de una exponencial a una normal truncada.

Hemos encontrado, para todos los escenarios, el tipo de distribución estadística que siguen las distribuciones del "tiempo hasta la interrupción" y del "tiempo de indisponibilidad" de cada punto de carga que compone la red subterránea:

- En todos los escenarios analizados, se puede ajustar la distribución del tiempo hasta la interrupción de los puntos de carga por una distribución exponencial.

- Respecto al ajuste de las distribuciones del tiempo de indisponibilidad de los puntos de carga. En los escenarios en los que consideramos como hipótesis de distribución de los tiempos de reparación de los elementos de la red una distribución exponencial, se puede ajustar por una suma de distribuciones exponenciales, excepto en el escenario SUB-1 (DETR) en el que se ajustaba mediante una función de tipo exponencial. En los escenarios en los que consideramos como hipótesis de distribución de los tiempos de reparación de los elementos de la red una distribución normal truncada, se puede ajustar por una suma de distribuciones normales. 



\subsubsection{RED AÉREA}

Como ya se enunció en el apartado 3.1.2, los escenarios de este análisis se centrará en los efectos de las condiciones meteorológicas sobre los puntos de carga de la red. Para esto contamos con dos escenarios que, al igual que con la red subterránea, se utilizarán en dos ocasiones, una, suponiendo que la hipótesis de distribución de los tiempos de reparación de los elementos de la red sigue una distribución exponencial, y, otra, una normal truncada.

Se han clasificado las condiciones meteorológicas en dos tipos: bueno y malo, y como es usual en este trabajo, sus distribuciones de probabilidad se caracterizan por sendas exponenciales cuyas medias denominamos abreviadamente, $N$, para el buen tiempo, y $S$, para el mal tiempo. Por tanto, $N$ es la duración media de los ciclos de buen tiempo, y $S$ la de los ciclos de mal tiempo.

En la indisponibilidad de las redes aéreas, a diferencia de lo que ocurría en las subterráneas, sí que influyen las condiciones meteorológicas; porque condicionan el ciclo funcionamiento-fallo de todos o parte de sus elementos. Así sucede en nuestro caso, en el que las líneas aéreas, denominadas LA1, ven sus características de fiabilidad modificadas, toda vez que resultan, durante el mal tiempo, con una tasa de fallo 200 veces superior y un tiempo de reparación total igual a la suma del que tenían en buen tiempo y lo que resta de mal tiempo una vez que ha fallado, ya que las líneas aéreas no se reparan hasta que no cesa el mal tiempo (ver Anexo 2).

Para representar los resultados hemos elegido el punto de carga número "0", que se halla en el nudo de carga "18" en el centro de transformación $1^{\circ}$ (CT1), y el punto de carga número "10", situado en el nudo de carga "99" en el centro de transformación $11^{\circ}(\mathrm{CT} 11)$. No hay ninguna razón detrás de esta elección; podríamos haber elegido otros cualesquiera ya que los restantes puntos de carga se comportan cualitativamente de la misma manera que estos dos.

\section{DISTRIBUCIÓN EXPONENCIAL PARA EL TIEMPO DE REPARACIÓN (DETR) DE LOS ELEMENTOS DE LA RED.}

\section{Escenario AER-1 (DETR)}

Fijaremos una duración media para los ciclos de buen tiempo, $N$, de 400 horas, y variaremos la duración media para los ciclos de mal tiempo, $S$, para examinar las que repercusiones sobre las distribuciones de tiempo de indisponibilidad y tiempo hasta la interrupción de los puntos carga. Utilizaremos valores para $S$ de 0,2 y 20 horas.

En las figuras 4.34 y 4.35 se aprecia, cómo al aumentar la duración media de los ciclos de mal tiempo, $S$, la distribución del tiempo de indisponibilidad se desliza hacia interrupciones de mayor duración. Este efecto es debido a que al aumentar $S$ 
estamos aumentando el tiempo de reparación de todos los elementos que fallen en mal tiempo, ya que no se reparan hasta que no finaliza tal evento. Esta influencia es, sobre todo, debida a las líneas aéreas LA1, ya que es el elemento que, con mucho, posee la mayor tasa de fallo en mal tiempo (500 veces superior a cualquiera de los demás elementos), y por tanto el que mayor número de interrupciones provoca.

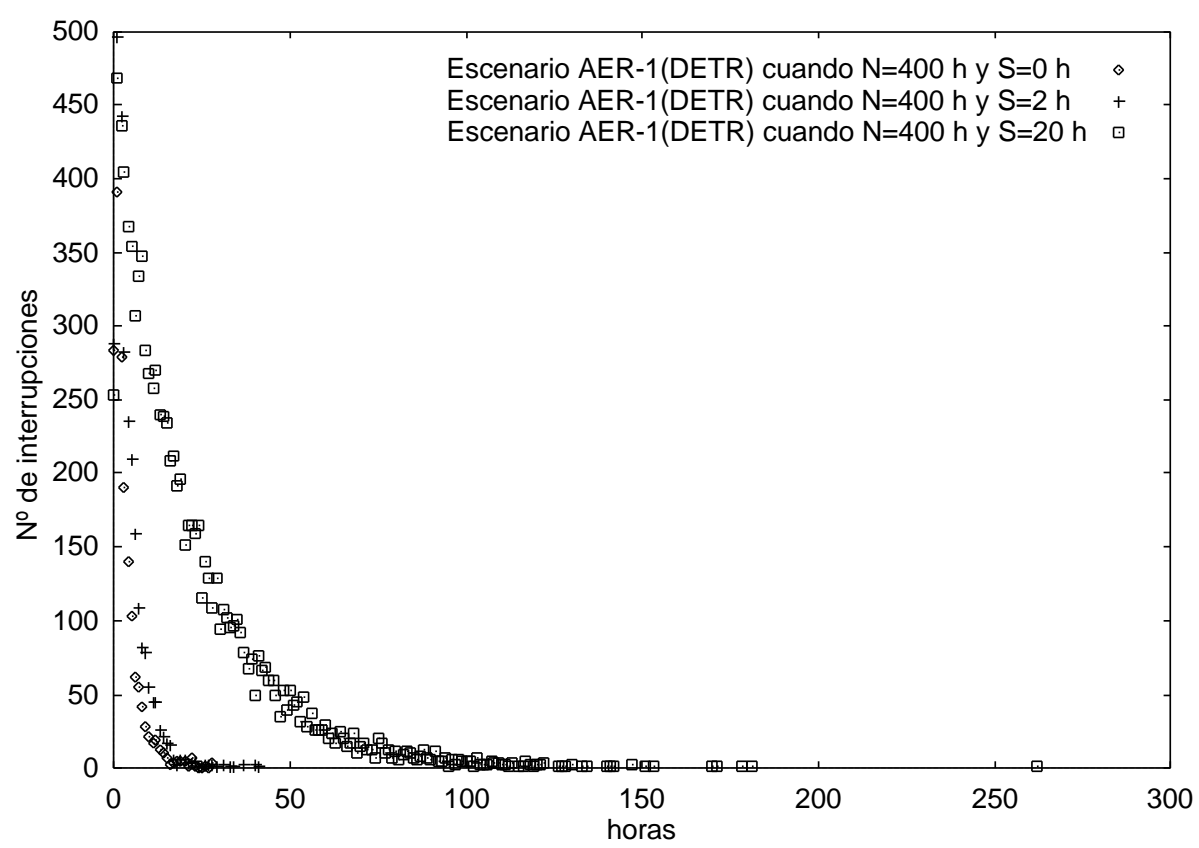

Fig.4.34.-Distribuciones del tiempo de indisponibilidad para el punto de carga número " 0 " de la red aérea cuando se asigna a $S$ valores de 0,2 y 20 horas.

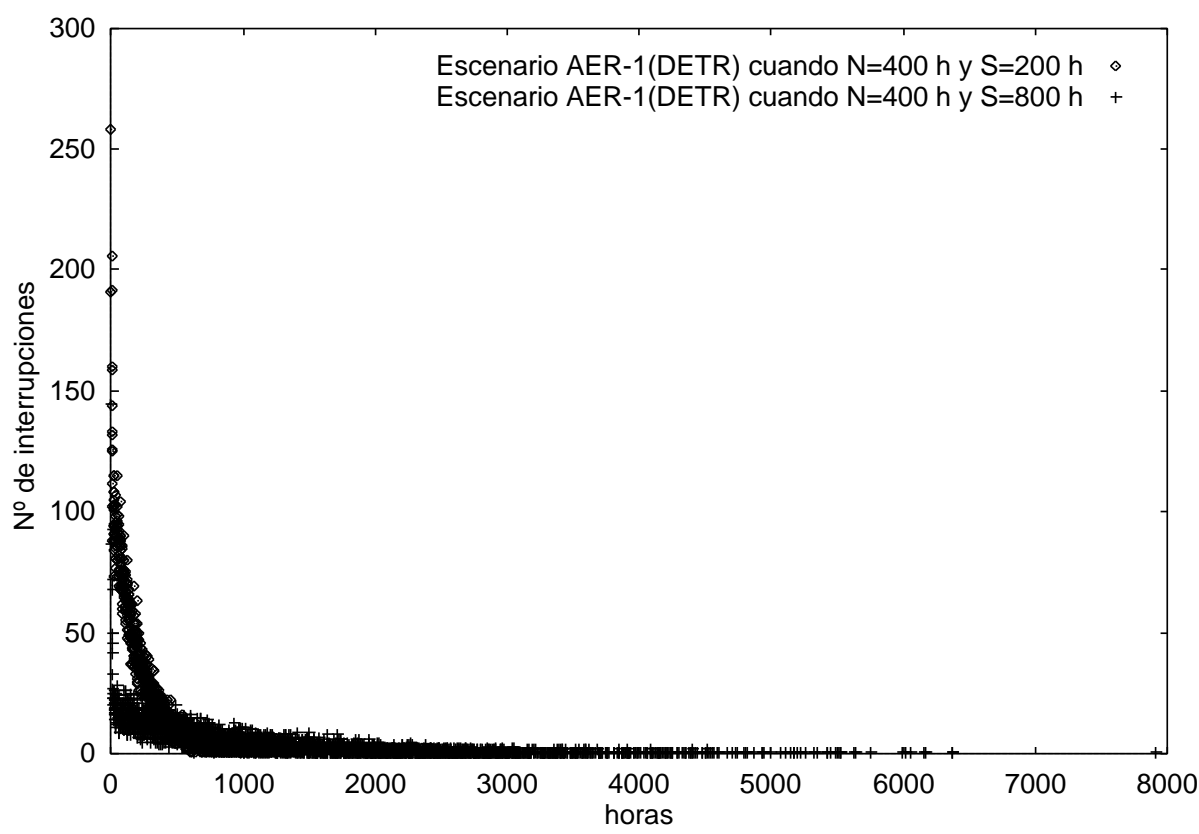

Fig.4.35.-Distribuciones del tiempo de indisponibilidad para el punto de carga número " 0 " de la red aérea cuando se asigna a $S$ valores de 200 y 800 horas. 


\section{CAPÍTULO 4 - RESULTADOS}

Aumentando la duración media de los ciclos de mal tiempo, $S$, aumenta el límite superior de la duración de las interrupciones, tal y como se representa en la figura 4.36.

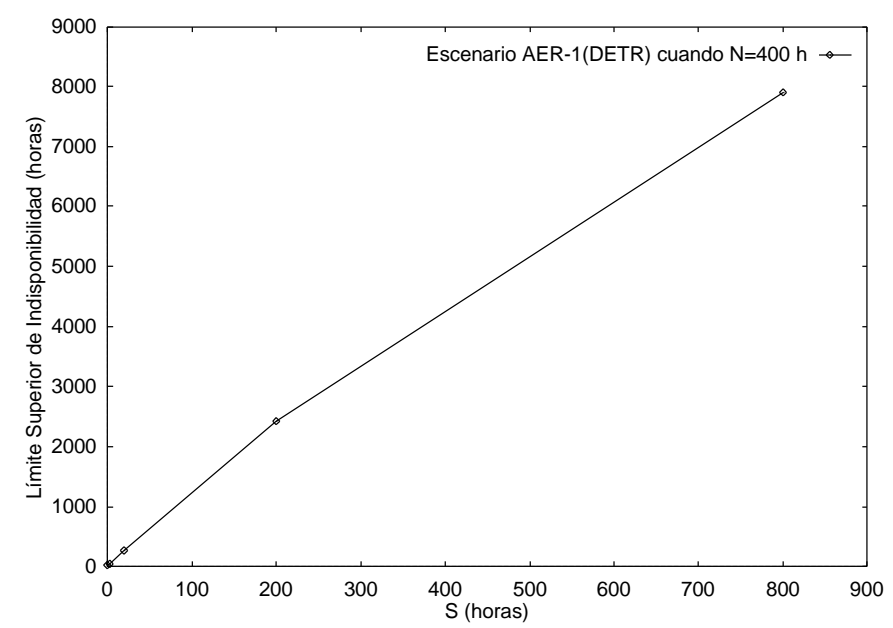

\begin{tabular}{|c|c|}
\hline $\begin{array}{c}S \\
\text { (horas) }\end{array}$ & $\begin{array}{c}\text { Límite Superior de } \\
\text { Indisponibilidad (horas) }\end{array}$ \\
\hline \hline 0 & 28 \\
\hline 2 & 41 \\
\hline 20 & 262 \\
\hline 200 & 2426 \\
\hline 800 & 7912 \\
\hline
\end{tabular}

Fig.4.36.-Evolución del límite superior de indisponibilidad para el punto de carga número " 0 " de la red aérea cuando se asigna a $S$ valores de $0,2,20,200$ y 800 horas.

En la figura 4.37 se observa cómo la distribución del tiempo de indisponibilidad se ajusta bien por una exponencial para un valor de $N$ igual a 400 horas y de $S$ igual a 20 horas. Este resultado es válido para valores de $S$ comprendidos entre 0 y 100 horas y para cualquier punto de carga.

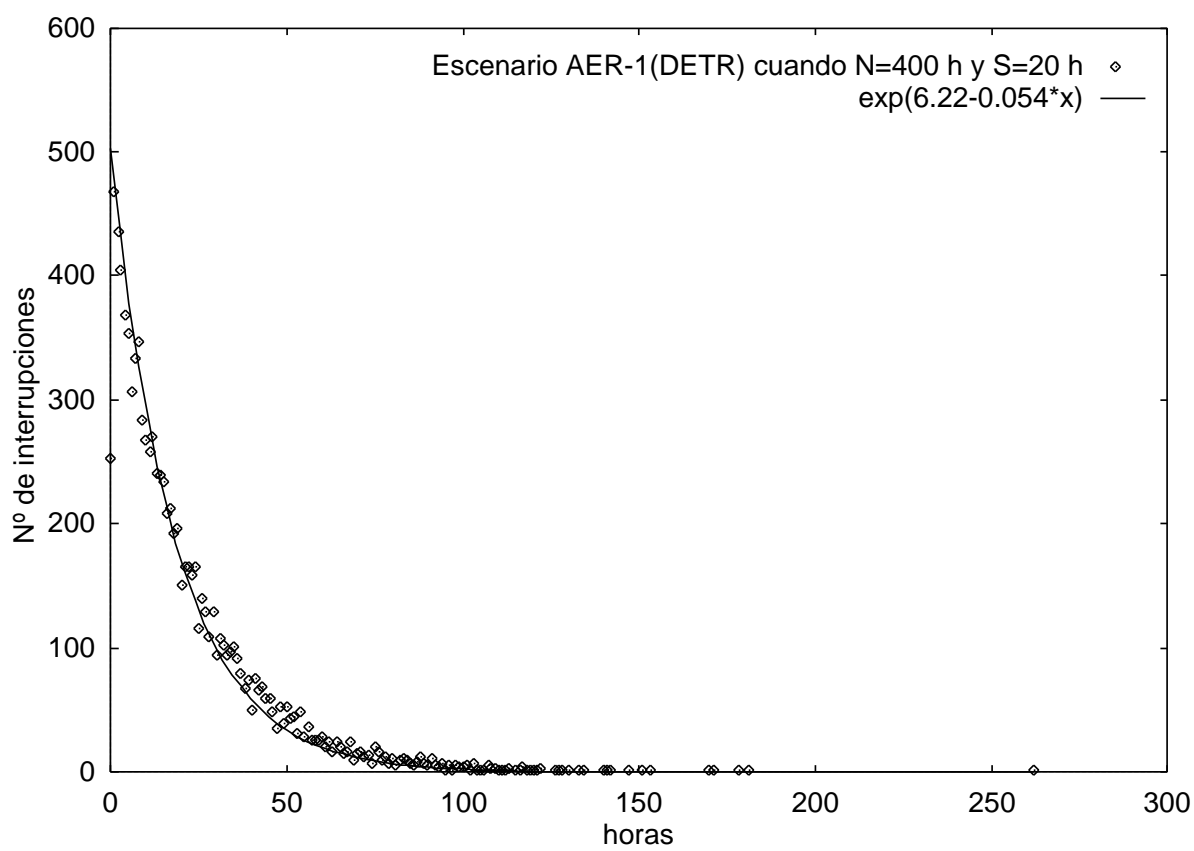

Fig.4.37.-Ajuste por una función exponencial de la distribución del tiempo de indisponibilidad para el punto de carga número " 0 " de la red aérea para el caso en el que $N=400$ horas y $S=20$ horas. 
Sin embargo, cuando los valores de la duración media de los ciclos de mal tiempo, $S$, superan las 100 horas, algo inusual, pues supone duraciones medias de los ciclos de mal tiempo de más de cuatro días de forma ininterrumpida, se debe emplear la suma de dos exponenciales como mejor ajuste

$$
f(x)=e^{(a-b x)}+e^{\left(a^{\prime}-b^{\prime} x\right)}
$$

como puede comprobarse en la figura 4.38.

Esta conclusión es general para cualquier punto de carga.
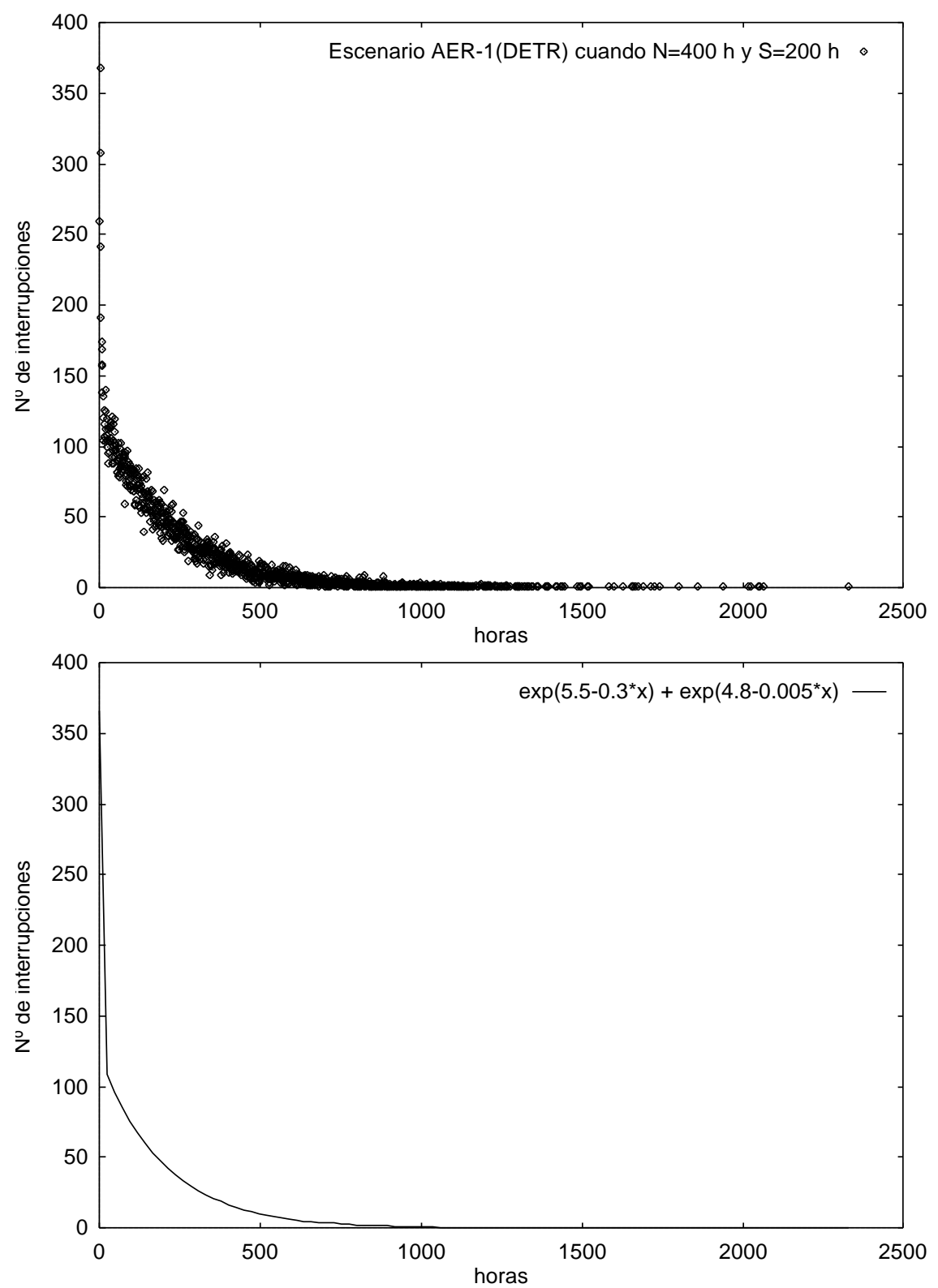

Fig.4.38.-Ajuste por una función suma de dos exponenciales de la distribución del tiempo de indisponibilidad para el punto de carga número 10 de la red aérea para el caso en el que $N=400$ horas y $S=200$ horas. 
En las figuras 4.39 y 4.40 se aprecia, cómo según aumenta $S$, se producen un mayor número de interrupciones. Esto es debido a que al aumentar la duración media de los ciclos de mal tiempo estamos dando más tiempo a los distintos elementos a que fallen, por tanto aumenta el número total de interrupciones y la distribución de tiempo hasta la interrupción se desliza hacia tiempos menores. Como consecuencia de esto, disminuyen los límites superior e inferior de continuidad de suministro, tendencia que se refleja en la figura 4.41.

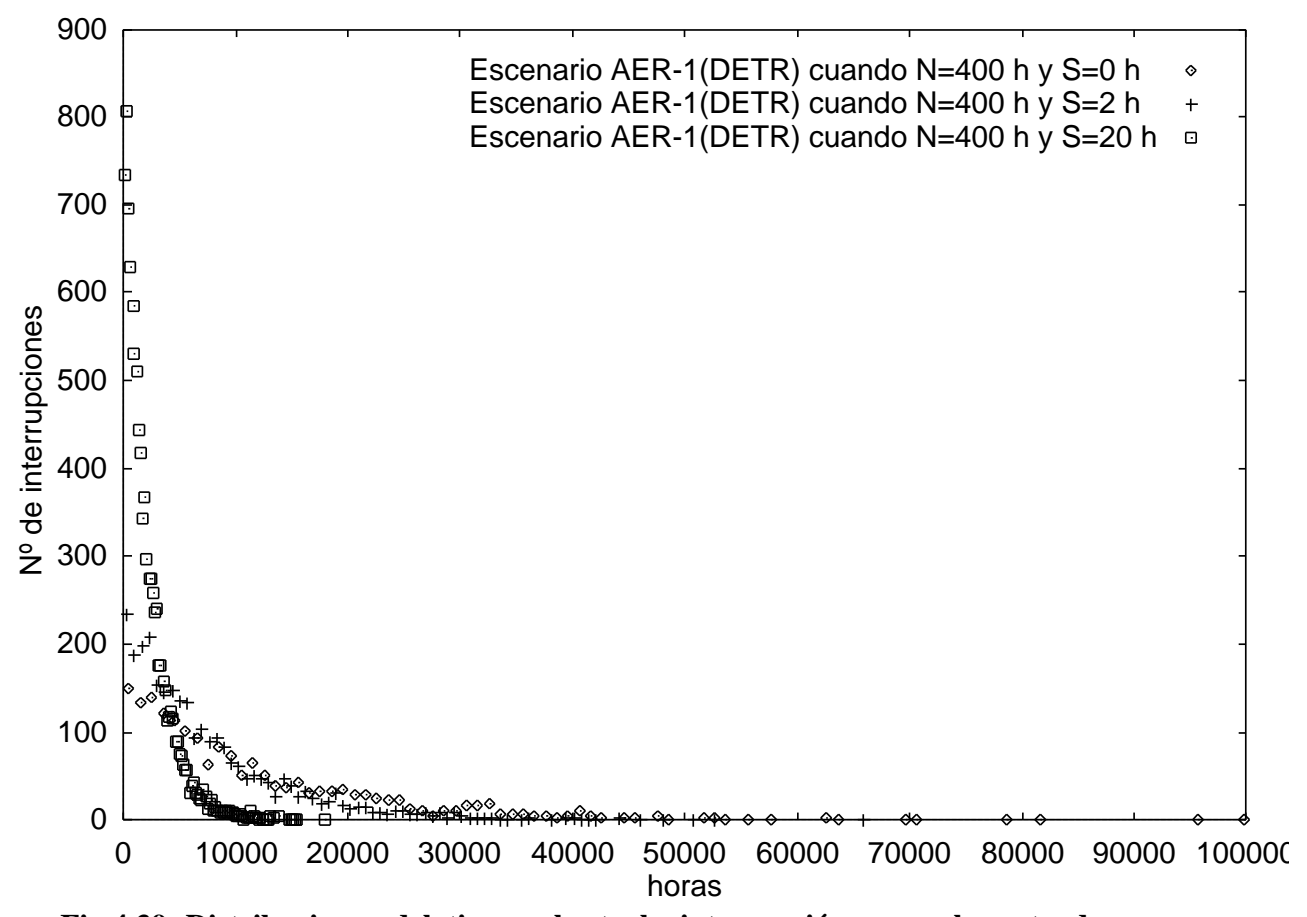

Fig.4.39.-Distribuciones del tiempo hasta la interrupción para el punto de carga número " 0 " de la red aérea cuando se asigna a $S$ valores de 0,2 y 20 horas.

Si continuamos aumentando la duración media de los ciclos de mal tiempo, $S$, hasta superar la de los ciclos de buen tiempo, $N$, llega un momento en el que desciende el número de interrupciones, como se recoge en la figura 4.40. A este resultado también llegamos en el escenario IND-3 del apartado 4.2. Esto es debido a que, mientras dura el mal tiempo, no se puede reponer el servicio, permaneciendo los elementos cada vez más tiempo en el estado de fallo sin posibilidad de ser reparados y por tanto con imposibilidad de fallar.

La figura 4.41 recoge la disminución de los límites superior e inferior de continuidad de suministro respecto al aumento en la duración media de los ciclos de mal tiempo, $S$. También se observa, para el valor de $\mathrm{S}$ de 800 horas el efecto anteriormente descrito para la figura 4.40 . 


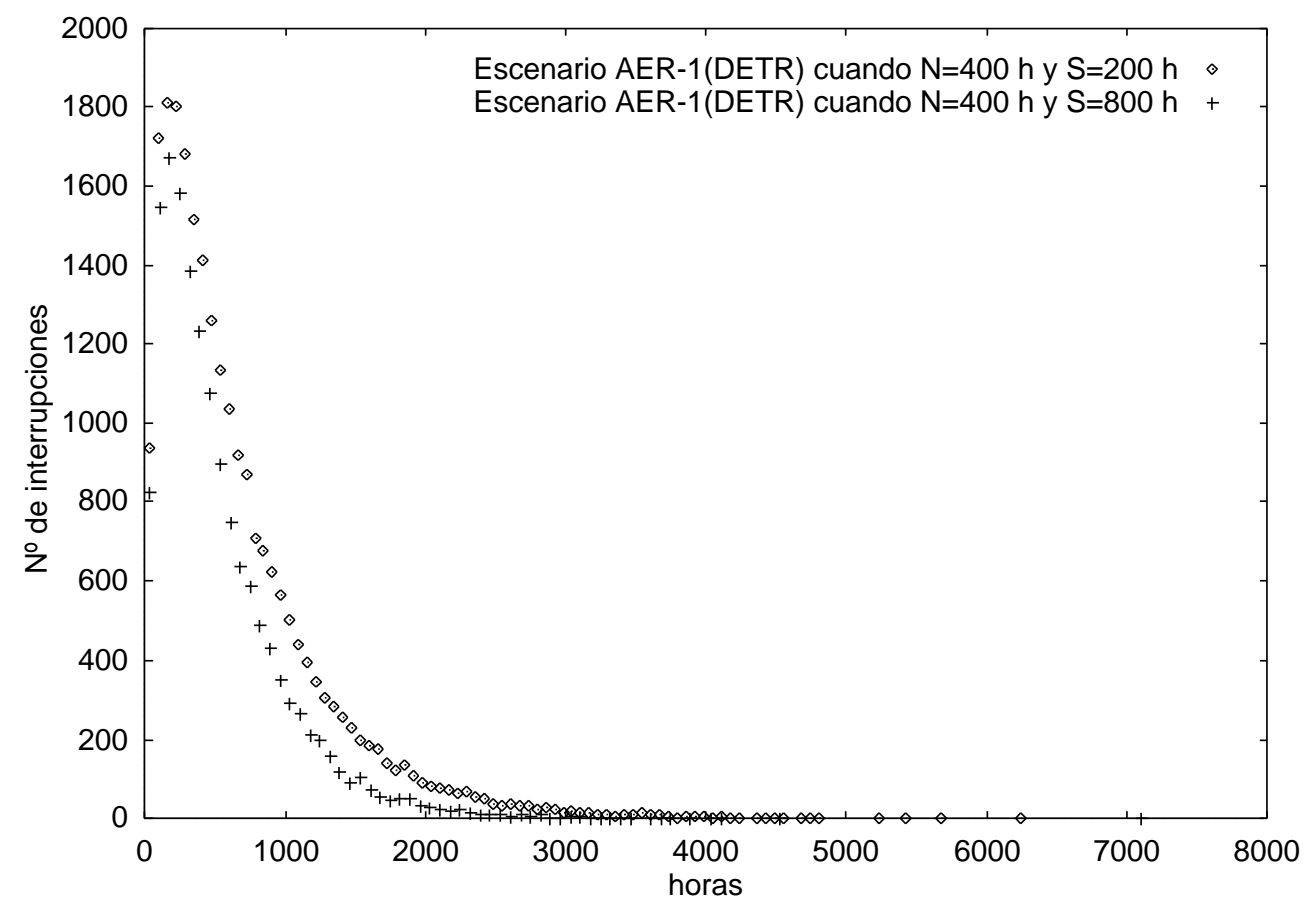

Fig.4.40.-Distribuciones del tiempo hasta la interrupción para el punto de carga número " 0 " de la red aérea cuando se asigna a $S$ valores de 200 y 800 horas.

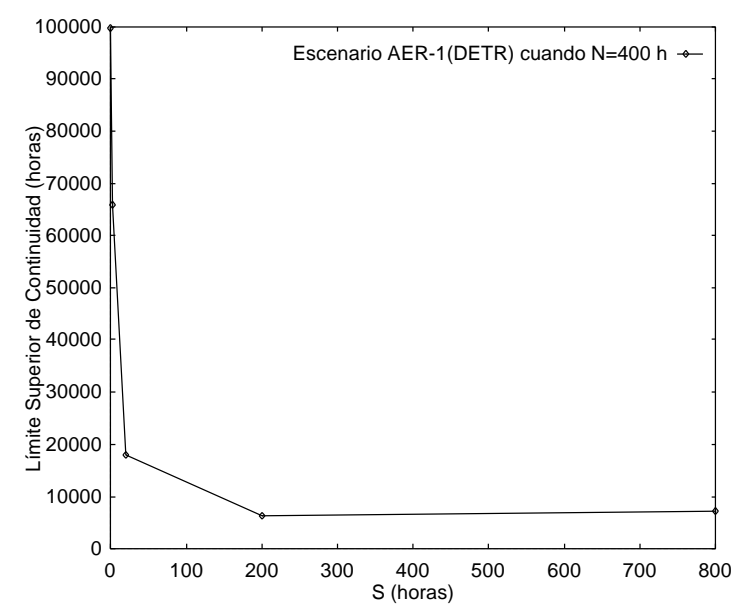

\begin{tabular}{|c|c|c|}
\hline $\begin{array}{c}\text { S } \\
\text { (horas) }\end{array}$ & $\begin{array}{c}\text { Límite Superior de } \\
\text { Continuidad (horas) }\end{array}$ & $\begin{array}{c}\text { Límite Inferior de } \\
\text { Continuidad (horas) }\end{array}$ \\
\hline \hline 0 & 99.747 & 507 \\
\hline 2 & 65.946 & 332 \\
\hline 20 & 17.979 & 90 \\
\hline 200 & 6.242 & 31 \\
\hline 800 & 7.102 & 36 \\
\hline
\end{tabular}

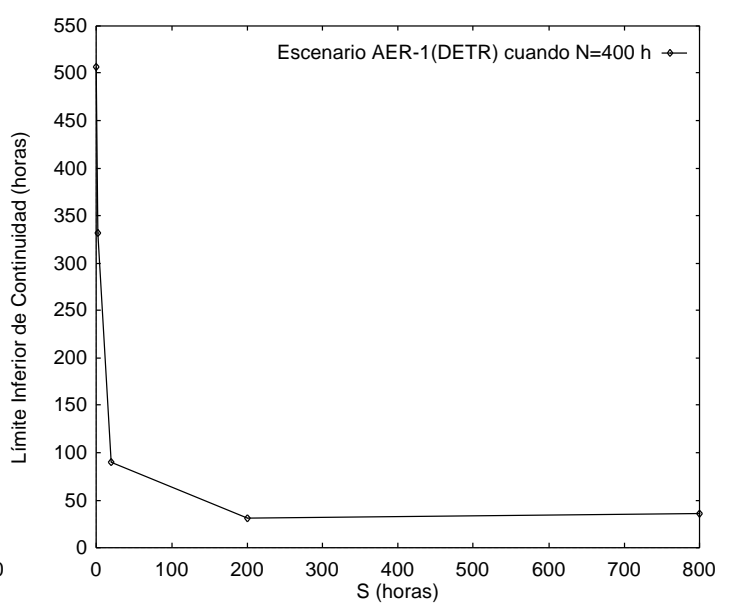

Fig.4.41.-Evolución del límite superior e inferior de continuidad de suministro para el punto de carga número " 0 " de la red aérea cuando se asigna a $S$ valores de 0 , 2, 20, 200 y 800 horas. 
La distribución de tiempo hasta la interrupción del punto de carga número "0" para $N=400$ h y $S=20$ h se puede ajustar por una función exponencial

$$
f(x)=e^{(a-b x)}
$$

como puede verificarse en la figura 4.42 .

Como en el caso de la red subterránea, es fácil comprobar la generalidad de este resultado para todos los puntos de carga, en los dos escenarios contemplados y con cualquiera de los dos tipos de distribución que, para el tiempo de reparación de los elementos de la red, se han empleado.

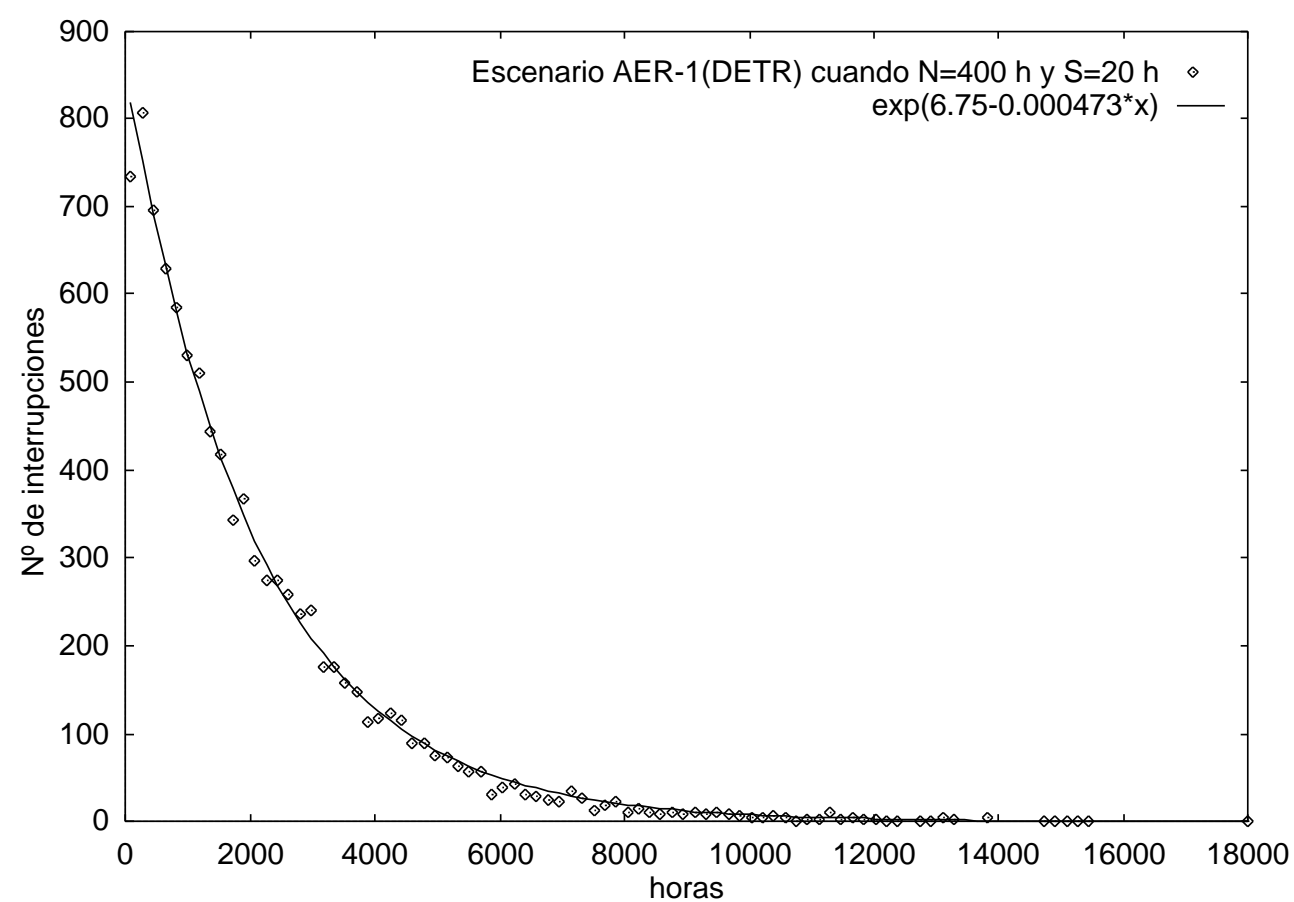

Fig.4.42.-Ajuste por una función exponencial de la distribución del tiempo hasta la interrupción para el punto de carga número " 0 " de la red aérea cuando $N$ $=400$ horas y $S=20$ horas.

\section{Escenario AER-2 (DETR)}

Fijemos ahora, en dos horas, la duración media $S$ de los ciclos de mal tiempo, y variemos la duración media de los ciclos de buen tiempo, $N$. Se trata de intentar apreciar el efecto resultante en las distribuciones de tiempo de indisponibilidad y tiempo hasta la interrupción.

La disminución del valor de $N$ no repercute en la forma de la ley de distribución del tiempo de indisponibilidad; aumenta el número de interrupciones, pero no la forma de distribuirse, como se puede comprobar en la figura 4.43. 
Aunque, como vimos en el apartado 4.1.2., si $N$ fuese menor que el tiempo medio de reparación de los elementos que componen la red (4 horas para las LA1), sí que aumentaría la duración de las interrupciones, dado que resultarían demasiado pequeños los períodos de buen tiempo para poder reponer el servicio.

El aumento del número de interrupciones es debido a que los fallos de los elementos se producen mayoritariamente en mal tiempo, y al disminuir la duración media de los ciclos del buen tiempo estamos aumentando la proporción de mal tiempo en cualquier intervalo de tiempo que se considere.

También, se puede ver, en la figura 4.43, que las duraciones de los tiempos de indisponibilidad no aumentan apreciablemente pues se mantiene constante el valor de $S$. Sin embargo, el límite superior del tiempo de indisponibilidad aumenta de 35 a 70 horas. Esto es debido a que, al existir más interrupciones, existen mayores frecuencias de aparición en todos los valores de indisponibilidad. Para que no hubiese aumentado el límite superior del tiempo de indisponibilidad, deberíamos haber utilizado una distribución exponencial truncada para los tiempos de reparación de los elementos que forman la red.

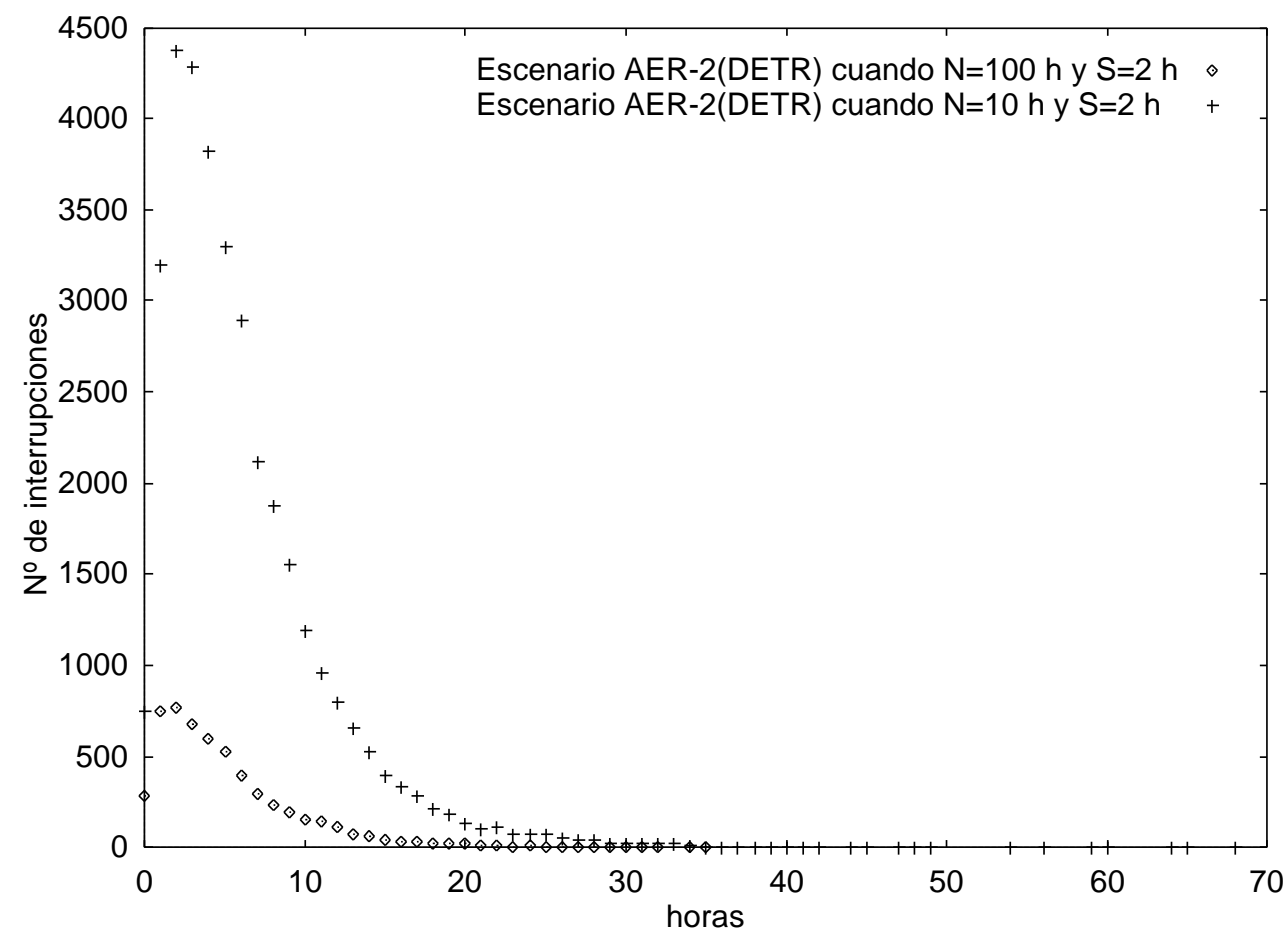

Fig.4.43.-Distribuciones del tiempo de indisponibilidad para el punto de carga número " 0 " de la red aérea cuando se asigna a $N$ valores de 100 y 10 horas. 
En la figura 4.44, se pone de manifiesto que, al disminuir la duración del buen tiempo, el número de interrupciones total aumenta, desplazándose la distribución hacia tiempos hasta la interrupción menores. También disminuye el límite superior e inferior del tiempo de continuidad de suministro, que pasa de 25.900 y 130 horas, respectivamente, a 6.600 y 30 horas.

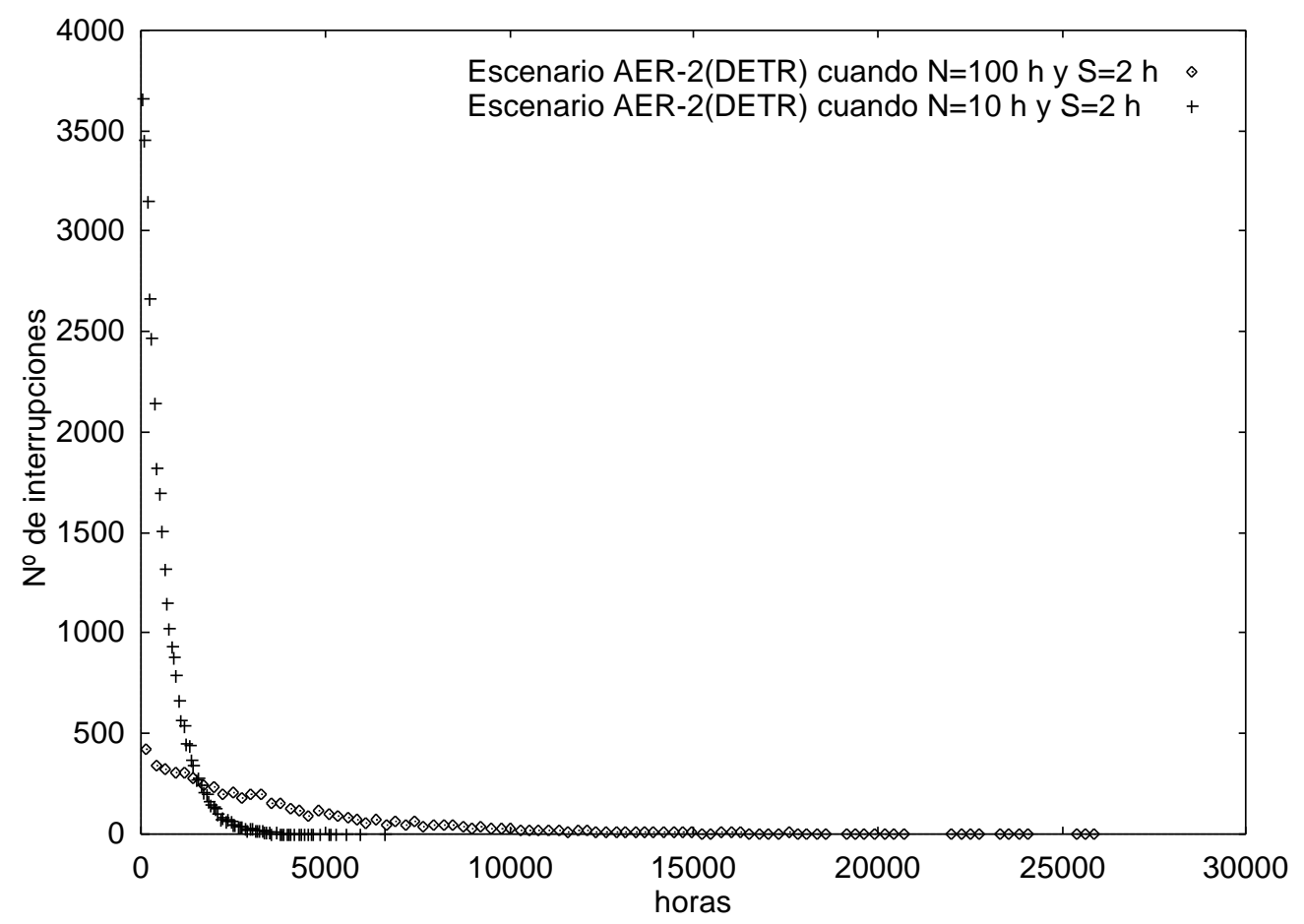

Fig.4.44.-Distribuciones del tiempo hasta la interrupción para el punto de carga número " 0 " de la red aérea cuando se asigna a $N$ valores de 100 y 10 horas.

Cuando mantenemos constante el valor de $S$ en 2 horas y disminuimos el valor de $N$ por debajo de las 100 horas, la distribución del tiempo de indisponibilidad deja de ser exponencial y se convierte en una distribución logarítmico-normal

$$
f(x)=\frac{1}{x \sigma \sqrt{2 \pi}} e^{-\frac{(\ln x-\mu)^{2}}{2 \sigma^{2}}}
$$

como se puede apreciar en la figura 4.45.

Este resultado es general para cualquier punto de carga. 


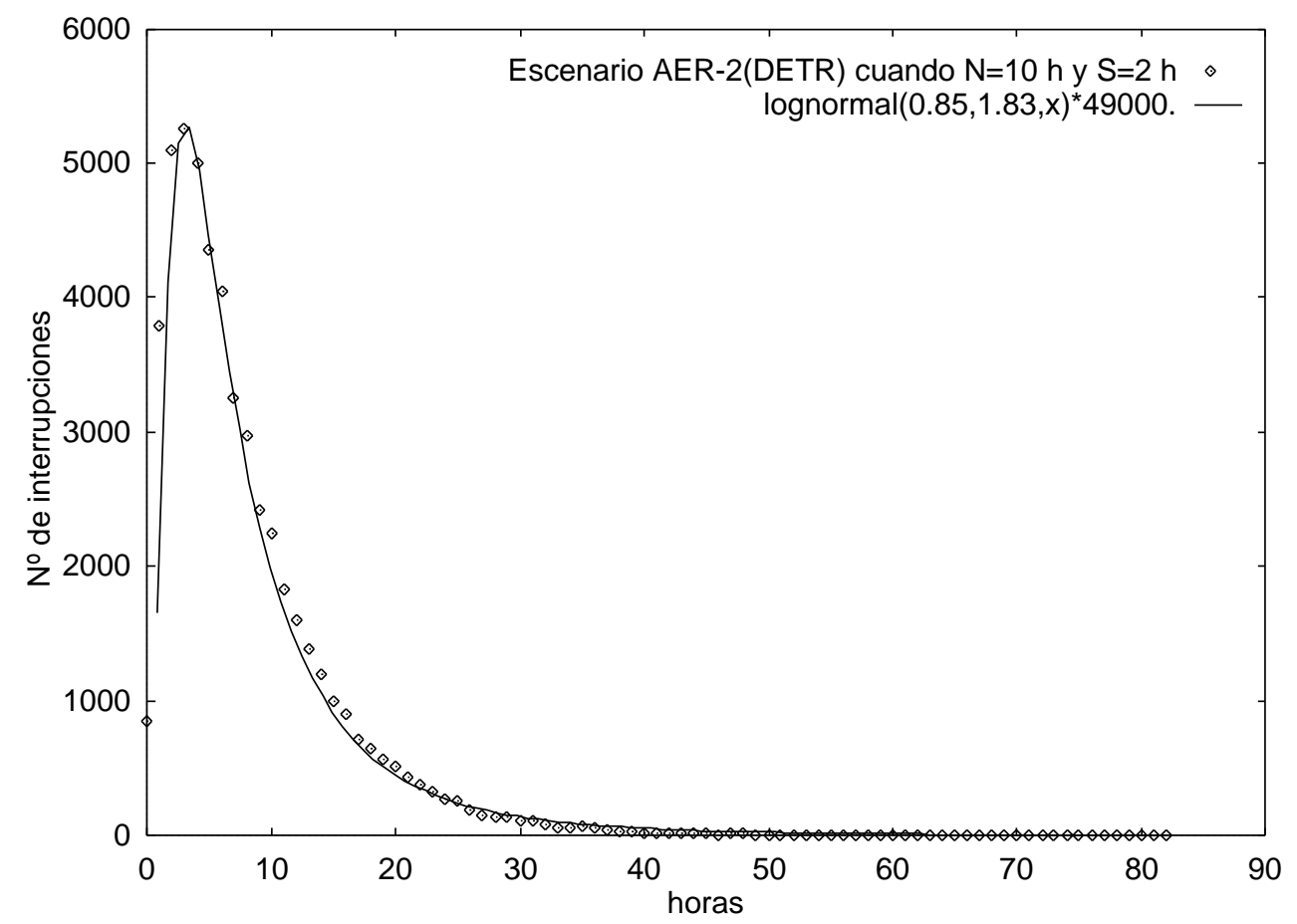

Fig.4.45.-Ajuste por una distribución logarítmico-normal de la distribución del tiempo de indisponibilidad para el punto de carga número 10 de la red aérea considerando que $N=10$ horas y $S=2$ horas.

\section{DISTRIBUCIÓN NORMAL PARA EL TIEMPO DE REPARACIÓN (DNTR) DE LOS ELEMENTOS DE LA RED.}

Consideremos ahora que la hipótesis de distribución de los tiempos de reparación de los elementos de la red, es que estén distribuidos siguiendo una normal truncada. Admitiremos que los valores para las tasas de fallo y tiempos de reparación de todos los elementos, y las duraciones medias del buen y mal tiempo $(N$ y $S$ ) que soportarán estos elementos serán los mismos que en los dos escenarios anteriores.

Al igual que sucedía con la red subterránea, es general el hecho de que en la ley de distribución del tiempo hasta la interrupción de cada punto de carga, solamente influya el tipo de distribución del tiempo hasta el fallo de los elementos que forman la red, y ésta en definitiva, es exponencial para todos los escenarios. Por consiguiente, es indiferente a estos efectos, que el tiempo de reparación obedezca a una distribución exponencial (DETR) o a una normal truncada (DNTR), pues el tiempo hasta la interrupción de cada punto de carga será sistemáticamente exponencial. No sucederá así con la ley de distribución del tiempo de indisponibilidad como veremos a continuación.

\section{Escenario AER-1 (DNTR)}


Fijaremos una duración media para los ciclos de buen tiempo, $N$, de 400 horas, y variaremos la media del mal tiempo para analizar las repercusiones en las distribuciones de tiempo de indisponibilidad y poder comparar con estrategias similares cuando considerábamos que los tiempos de reparación de cada uno de los elementos se distribuían exponencialmente.

En las figuras 4.46 y 4.47 se aprecia que las frecuencias de aparición de las distribuciones de tiempo de indisponibilidad casi no difieren de las obtenidas en los escenarios AER-1 (DETR) en que empleábamos tiempos de reparación exponenciales, siendo esta semejanza mayor cuanto más incrementamos el valor de $S$, véase figuras 4.34 y 4.34 .
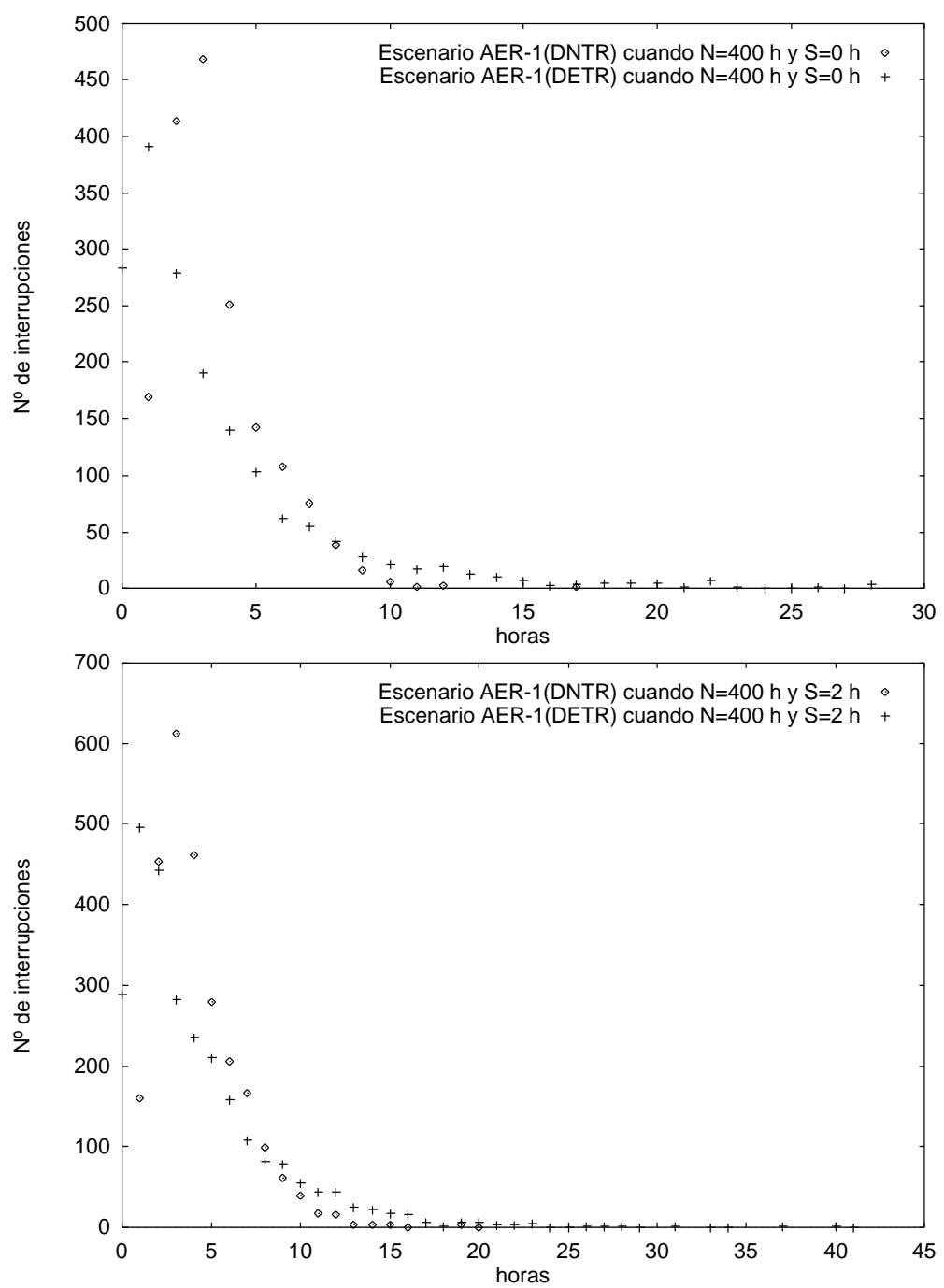

Fig.4.46.-Comparación entre las distribución del tiempo de indisponibilidad para el punto de carga número " 0 " de la red aérea en el supuesto en el que el tiempo de reparación de los elementos se considere distribuido exponencialmente o normalmente. 

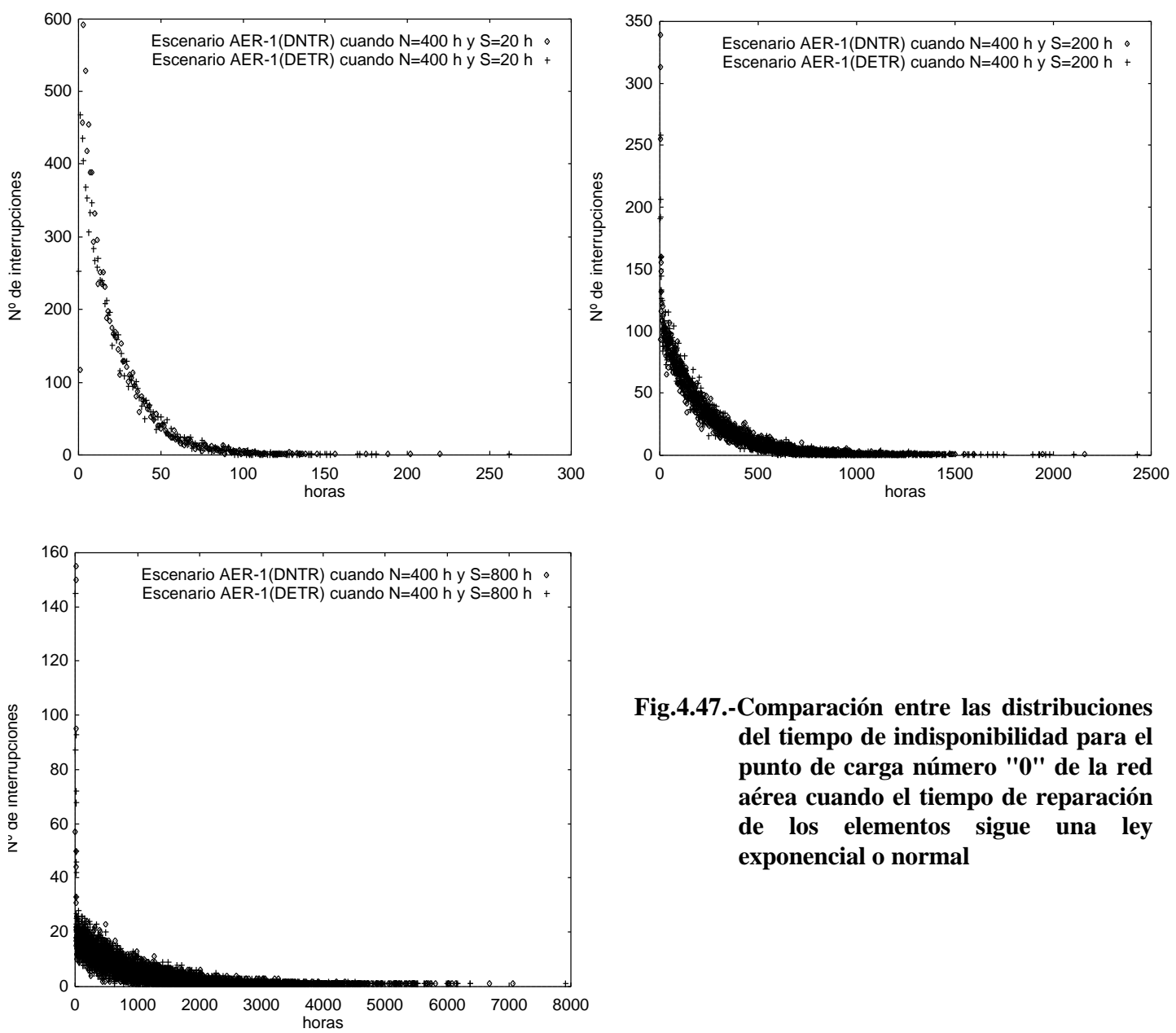

Fig.4.47.-Comparación entre las distribuciones del tiempo de indisponibilidad para el punto de carga número " 0 " de la red aérea cuando el tiempo de reparación de los elementos sigue una ley exponencial o normal

También se puede apreciar que los resultados presentan una dispersión menor que en sus equivalentes con tiempos de reparación exponenciales porque truncamos las colas de las distribuciones normales. Esto provoca que para $S$ igual a 0 horas y a 2 horas, el límite superior del tiempo de indisponibilidad en el escenario AER-1 (DNTR) disminuya a la mitad del valor que poseía en el escenario AER-1 (DETR). Para el resto de valores que asignamos a la duración media de los ciclos de mal tiempo, la diminución es apreciable aunque de menor magnitud, véase figura 4.47.

Como se aprecia en la figura 4.48, la distribución que se obtiene para el tiempo de indisponibilidad cuando $N$ es igual a 400 horas y $S$ igual a 20 horas, se ajusta perfectamente por una distribución suma de tres distribuciones normales.

Este resultado es general para cualquier punto de carga. 


\section{CAPÍTULO 4 - RESULTADOS}

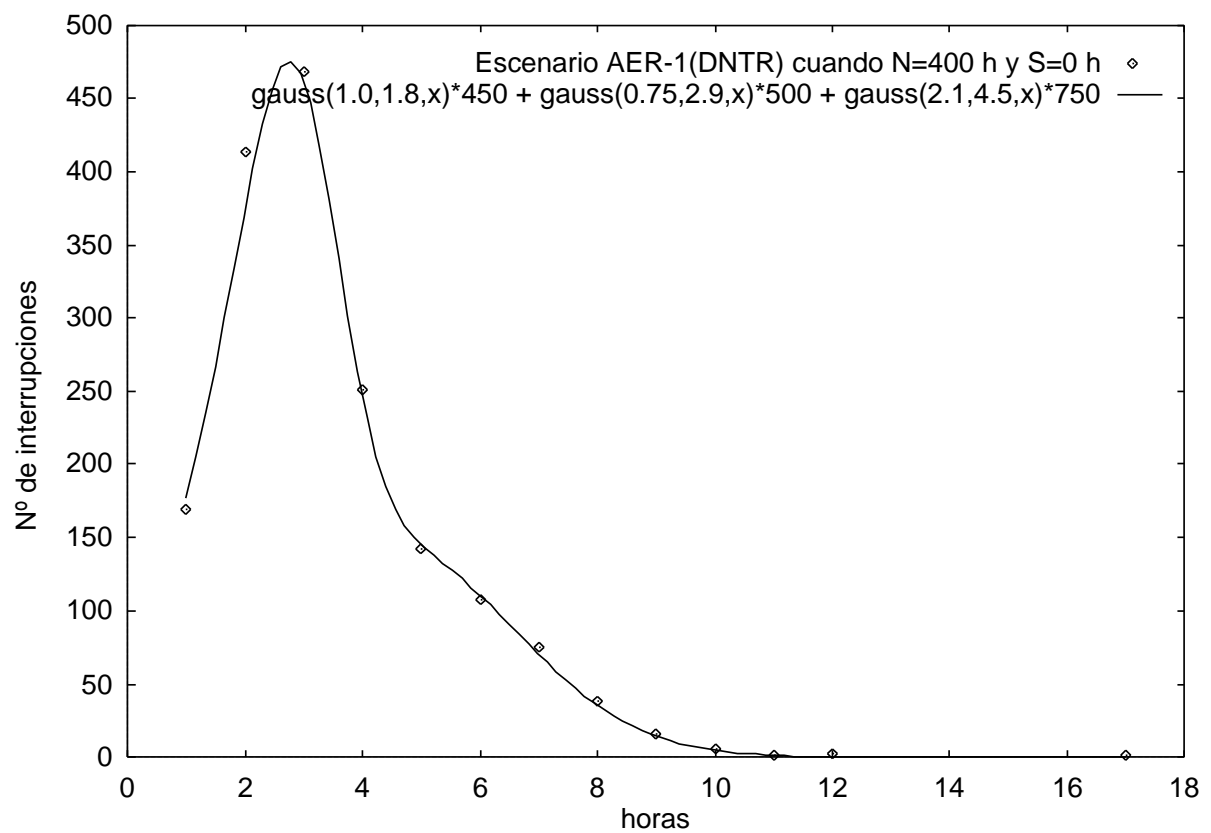

Fig.4.48.-Ajuste mediante una suma de tres distribuciones normales de la distribución del tiempo de indisponibilidad para el punto de carga número " 0 " de la red aérea cuando $N=400$ h y $S=0 \mathrm{~h}$.

Si continuamos aumentando la duración media $S$, de los ciclos de mal tiempo, la distribución obtenida para el tiempo de indisponibilidad, cuando $N$ vale 400 horas y $S 20$ horas, se ajusta perfectamente por una distribución logarítmico-normal, como puede comprobarse en la figura 4.49.

Este resultado es general para cualquier punto de carga.

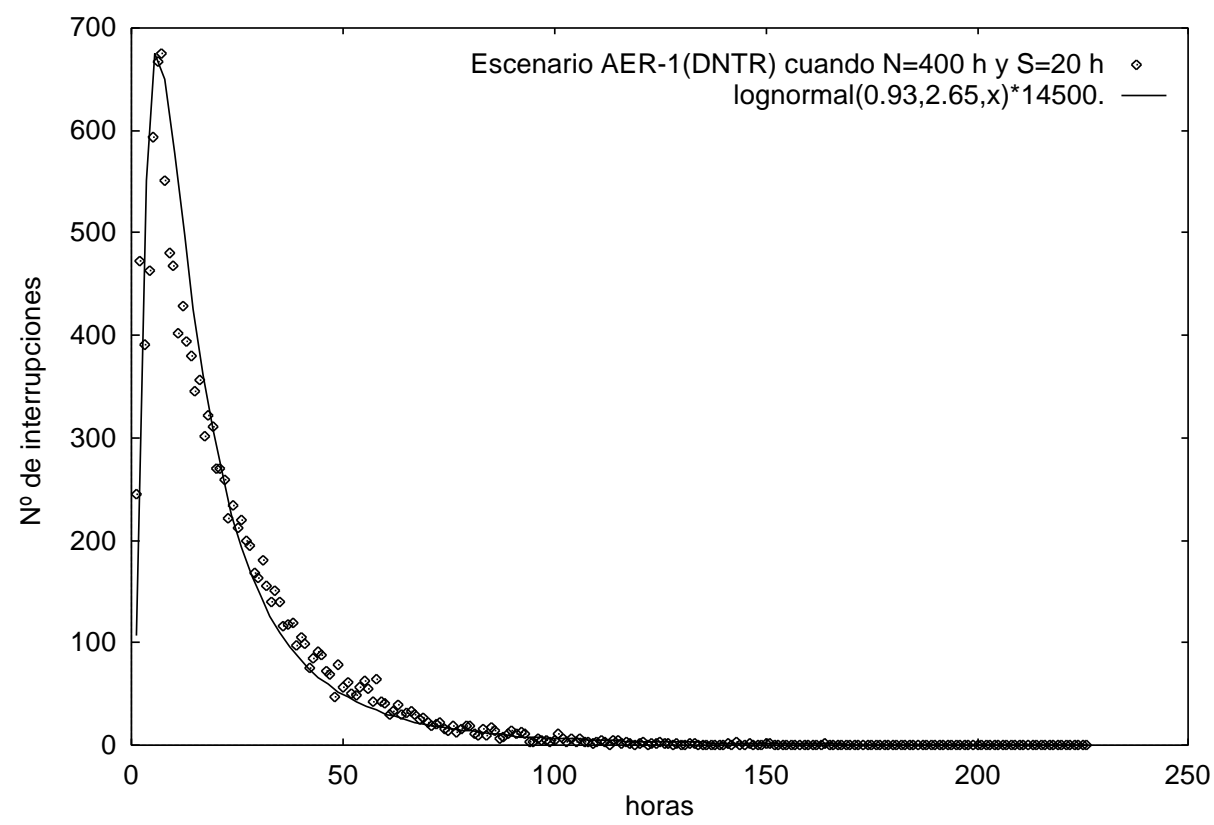

Fig.4.49.-Ajuste por una distribución logarítmico-normal de la distribución del tiempo de indisponibilidad para el punto de carga número "10" de la red aérea cuando $N=400$ horas y $S=20$ horas. 
Si continuamos aumentando la duración media de los ciclos de mal tiempo por encima de las 100 horas, la distribución de tiempo de indisponibilidad resultante es idéntica a la obtenida utilizando una distribución exponencial para el tiempo de reparación de los elementos, como ya se pudo observar en la figura 4.47. Por tanto, estas distribuciones se podrán ajustar por una distribución suma de dos distribuciones exponenciales, como quedó ya patente en la figura 4.38.

\section{Escenario AER-2 (DNTR)}

Ahora fijaremos la duración media de los ciclos de mal tiempo, $S$, en 2 horas, y variaremos la duración media de los ciclos buen tiempo, $N$, para poder apreciar los efectos que provoca en las distribuciones de tiempo de indisponibilidad y tiempo hasta la interrupción de los puntos de carga de la red y compararlos con los mismos cambios cuando considerábamos que los tiempos de reparación de los elementos se distribuían exponencialmente.

Como se puede observar en la figura 4.50, y al igual que sucedía en el escenario anterior, las distribuciones son muy parecidas a las obtenidas considerando tiempos de reparación exponencialmente distribuidos, aunque con una menor dispersión.
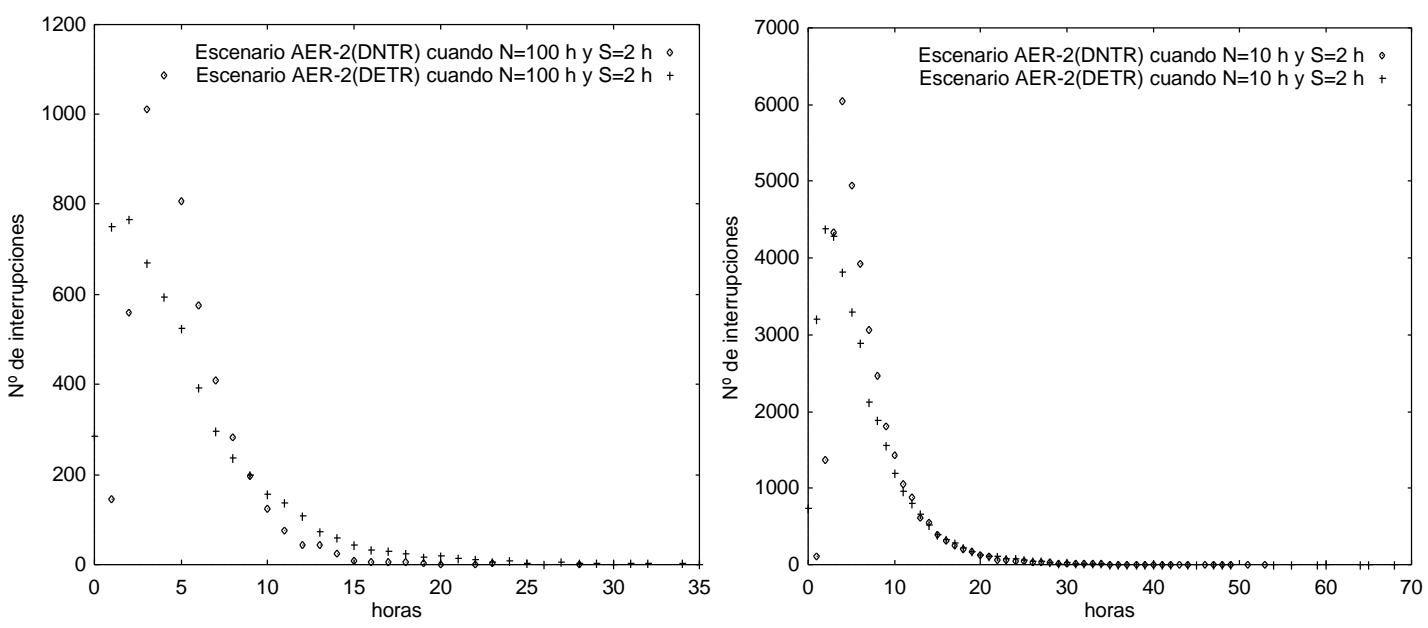

Fig.4.50.-Comparación entre las distribución del tiempo de indisponibilidad para el punto de carga número " 0 " de la red aérea en el supuesto en el que el tiempo de reparación de los elementos se considere distribuido exponencialmente o normalmente. 
Para un valor de $N$ igual a 10 horas y $S$ igual a 2 horas, la distribución que obtenemos para el tiempo de indisponibilidad se puede ajustar perfectamente por una distribución logarítmico-normal, como puede comprobarse en la figura 4.51. Este resultado es igual al obtenido cuando el tiempo de reparación de los elementos de la red se distribuía exponencialmente, como ya se puso de manifiesto en la figura 4.45.

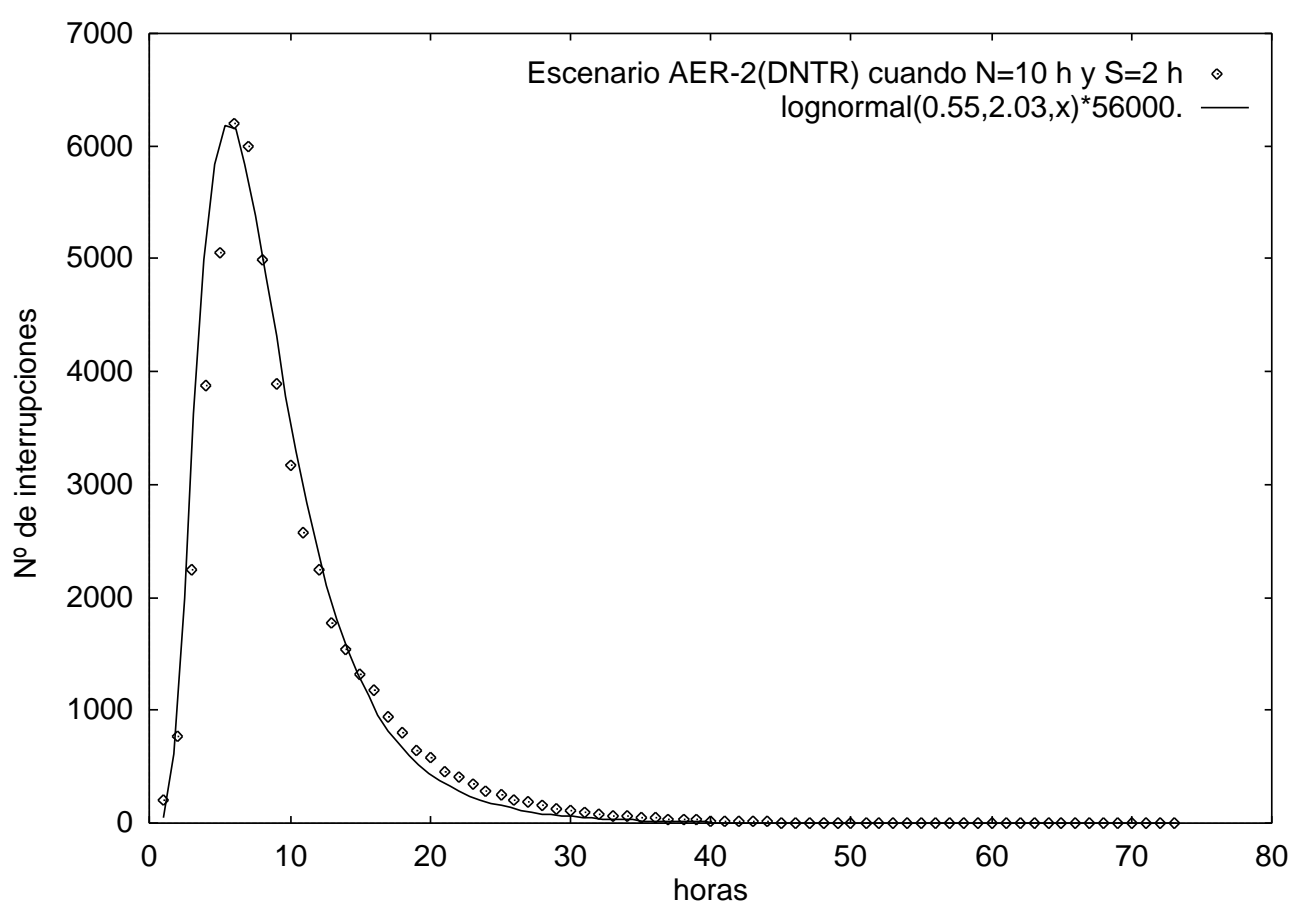

Fig.4.51.-Ajuste por una distribución logarítmico-normal de la distribución del tiempo de indisponibilidad para el punto de carga número "10" de la red aérea cuando $N=10$ horas y $S=2$ horas

\section{ASPECTOS A DESTACAR SOBRE LAS REDES AÉREAS}

Hemos hallado las distribuciones del tiempo hasta la interrupción y del tiempo de indisponibilidad en todos los escenarios analizados.

Hemos podido conocer los límites superior e inferior de las distribuciones del tiempo hasta la interrupción de los puntos de carga de la red en todos los escenarios analizados, éstos decrecen mientras disminuye la duración media de los ciclos de buen tiempo, $N$ y aumenta la duración media de los ciclos de mal tiempo, $S$.

También pudimos medir el límite superior de las distribuciones del tiempo de indisponibilidad, éste crece mientras aumenta el valor de $S$. Al cambiar el tipo de distribución para el tiempo de reparación de los elementos, y pasar de una exponencial a una normal truncada, disminuye el límite superior de la distribución del tiempo de indisponibilidad. 
Hemos encontrado, para todos los escenarios, el tipo de distribución estadística que siguen las distribuciones del "tiempo hasta la interrupción" y del "tiempo de indisponibilidad" de cada punto de carga que compone la red aérea:

- En todos los escenarios analizados, se puede ajustar la distribución del tiempo hasta la interrupción de los puntos de carga por una distribución exponencial.

- Respecto al ajuste de las distribuciones del tiempo de indisponibilidad de los puntos de carga. En los escenarios en los que consideramos como hipótesis de distribución de los tiempos de reparación de los elementos de la red una distribución exponencial, se puede ajustar, bien, por una distribución exponencial (p.ej. cuando $N=400 \mathrm{~h}$ y $S=20 \mathrm{~h}$ ), o una suma de dos exponenciales (p.ej. cuando $N=400 \mathrm{~h}$ y $S>100 \mathrm{~h}$ ), o una distribución logarítmico-normal (p.ej. cuando $N<100$ h y $S=2$ h). En los escenarios en los que consideramos como hipótesis de distribución de los tiempos de reparación de los elementos de la red una distribución normal truncada, se puede ajustar, bien, por una suma de distribuciones normales (p.ej. cuando $N=400 \mathrm{~h}$ y $S=0$ h), o por una distribución logarítmico-normal (p.ej. cuando $N=400$ h y $S=20$ h, o cuando $N=10$ h y $S=2$ h).

\subsubsection{RED MIXTA}

Como se enunció en el apartado 3.1.2, el siguiente análisis se centrará en el efecto de las condiciones meteorológicas sobre la indisponibilidad en los puntos de carga cuyo suministro dependa de algún tramo aéreo, manteniendo constantes las características de fiabilidad de los elementos de la red. Para esto contamos con dos escenarios idénticos a los utilizados para la red aérea.

Al igual que en las dos redes anteriores, los escenarios que se consideran se utilizarán suponiendo que la hipótesis de distribución de los tiempos de reparación de los elementos de la red sigue, indistintamente, una distribución exponencial o una normal truncada.

Para representar los resultados hemos elegido el punto de carga número "58", que se halla en el nudo de carga "310" en el centro de transformación 59 (CT317). No hay ninguna razón para elegir este punto de carga entre todos los que dependen de algún tramo aéreo; podríamos haber elegido otro cualesquiera ya que los restantes puntos de carga se comportan cualitativamente de la misma manera que éste.

\section{Escenario MIX-1 y MIX-2 (DETR)}

Los resultados obtenidos para estos dos escenarios son similares a los obtenidos en la red aérea.Y los resultados se muestran en las figuras 4.52, $4.53 \mathrm{y}$ 4.54 . 


\section{CAPÍTULO 4 - RESULTADOS}
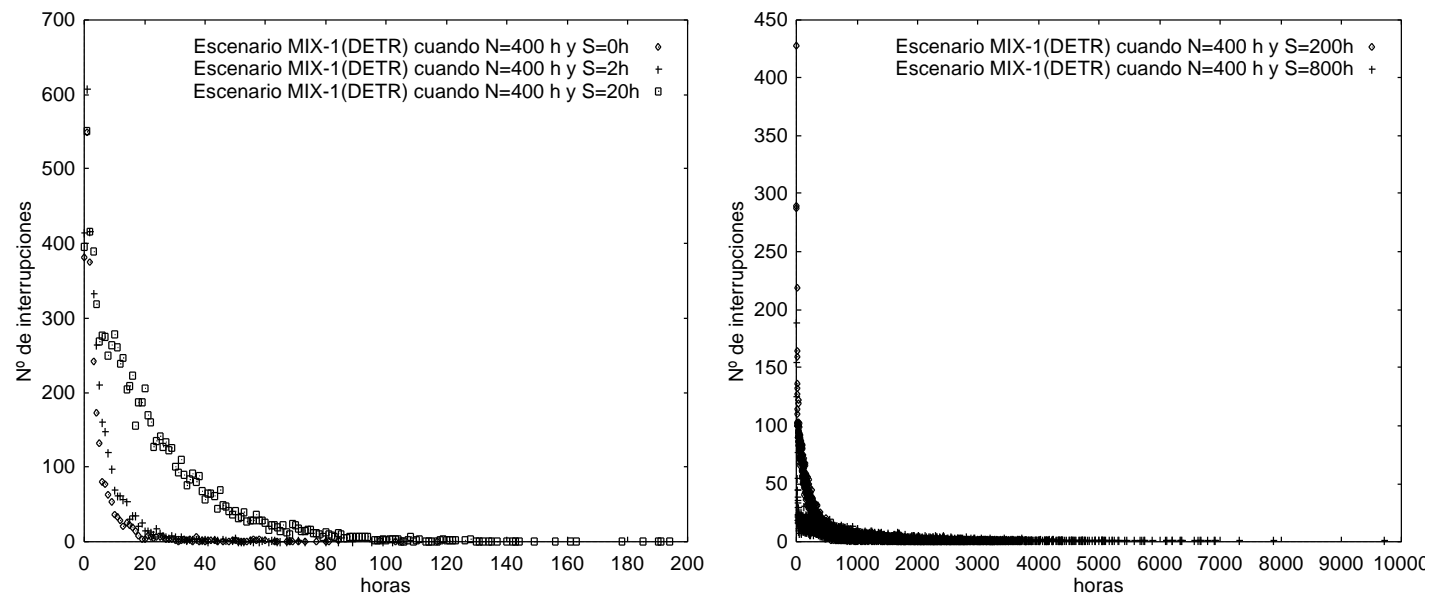

Fig.4.52.-Distribuciones del tiempo de indisponibilidad para el punto de carga número "58" de la red mixta cuando se asigna a $S$ valores de $0,2,20,200$ y 800 horas.
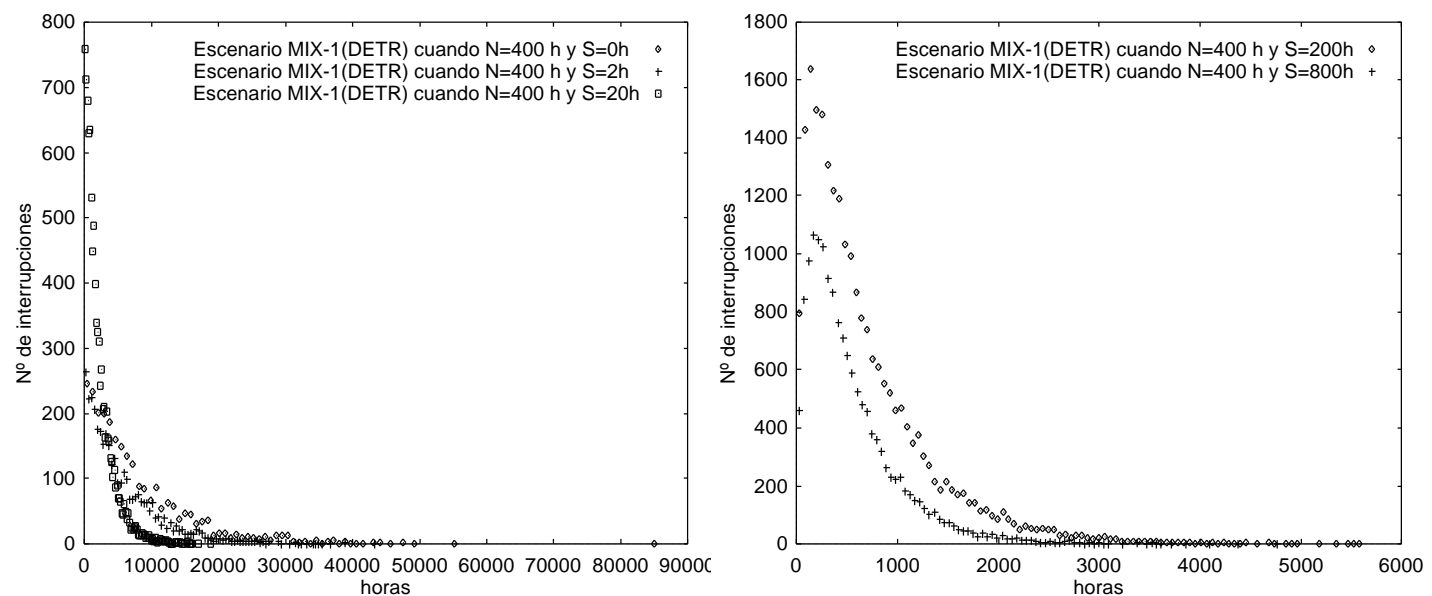

Fig.4.53.-Distribuciones del tiempo hasta la interrupción para el punto de carga número "58" de la red mixta cuando se asigna a $S$ valores de 0, 2, 20, 200 y 800 horas.
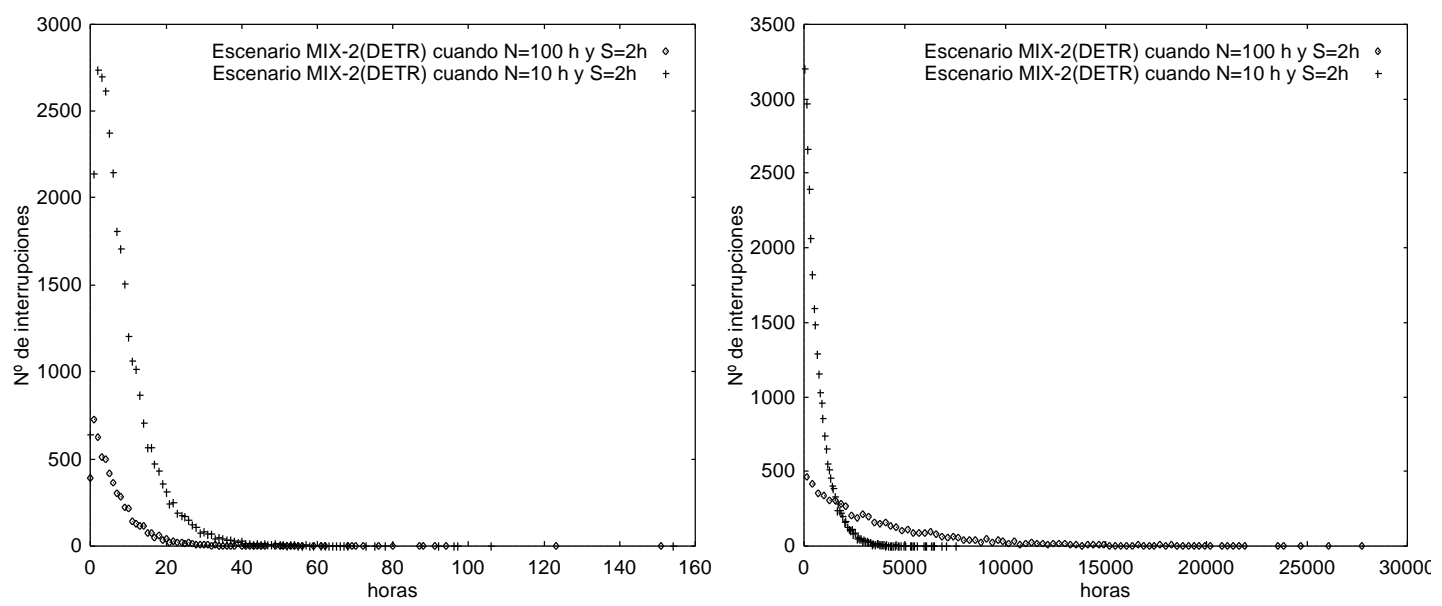

Fig.4.54.-Distribuciones del tiempo de indisponibilidad (figura de la izquierda) y tiempo hasta la interrupción (figura de la derecha) para el punto de carga número "58" de la red mixta cuando se asigna a $N$ valores de 100 y 10 horas. 


\section{Escenario MIX-1 y MIX-2 (DNTR)}

Al igual que en los dos escenarios anteriores, os resultados obtenidos son similares a los obtenidos en la red aérea. Los resultados se recogen en las figuras $4.55,4.56$ y 4.57 .
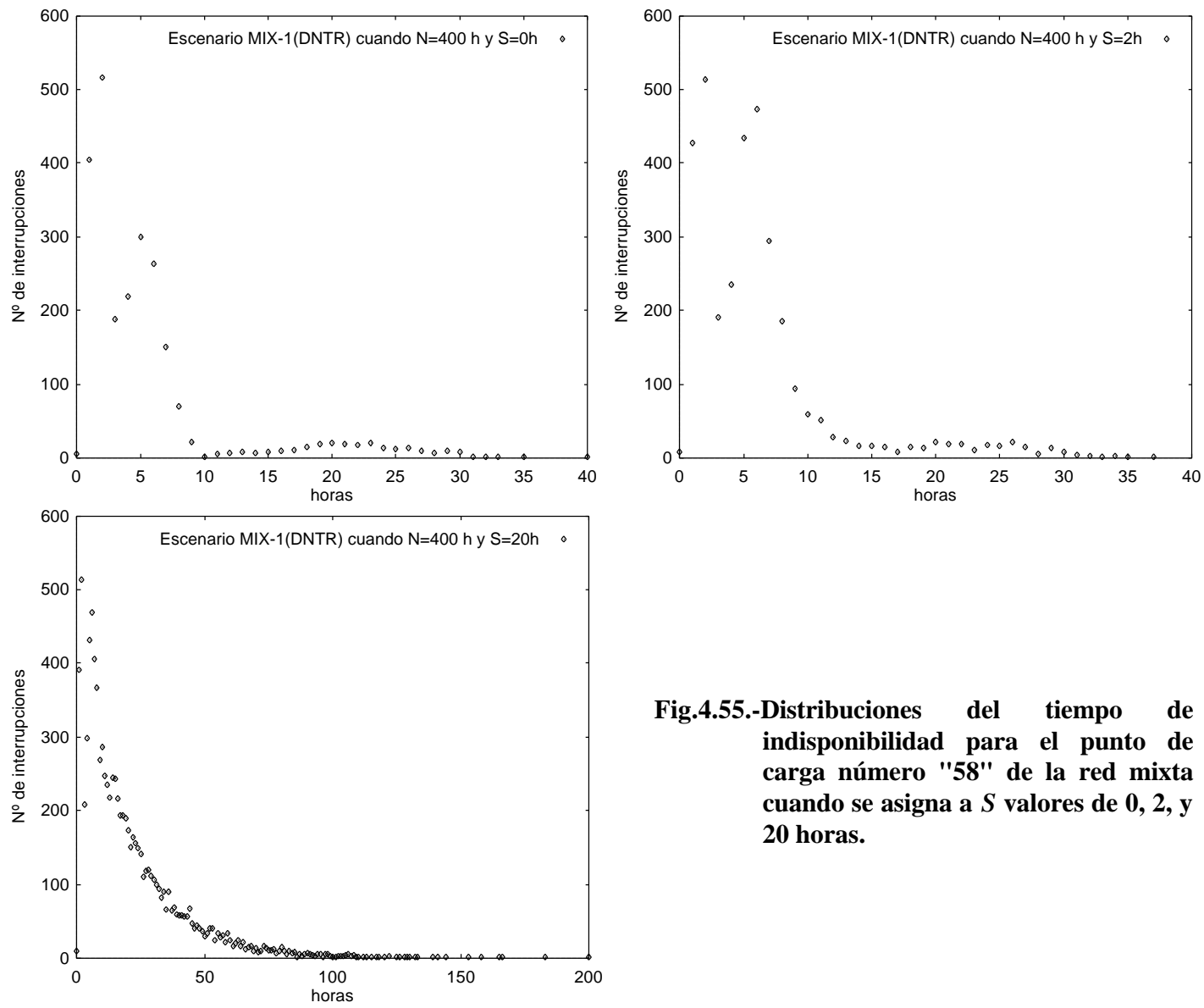

Fig.4.55.-Distribuciones del tiempo de indisponibilidad para el punto de carga número "58" de la red mixta cuando se asigna a $S$ valores de $0,2, y$ 20 horas.
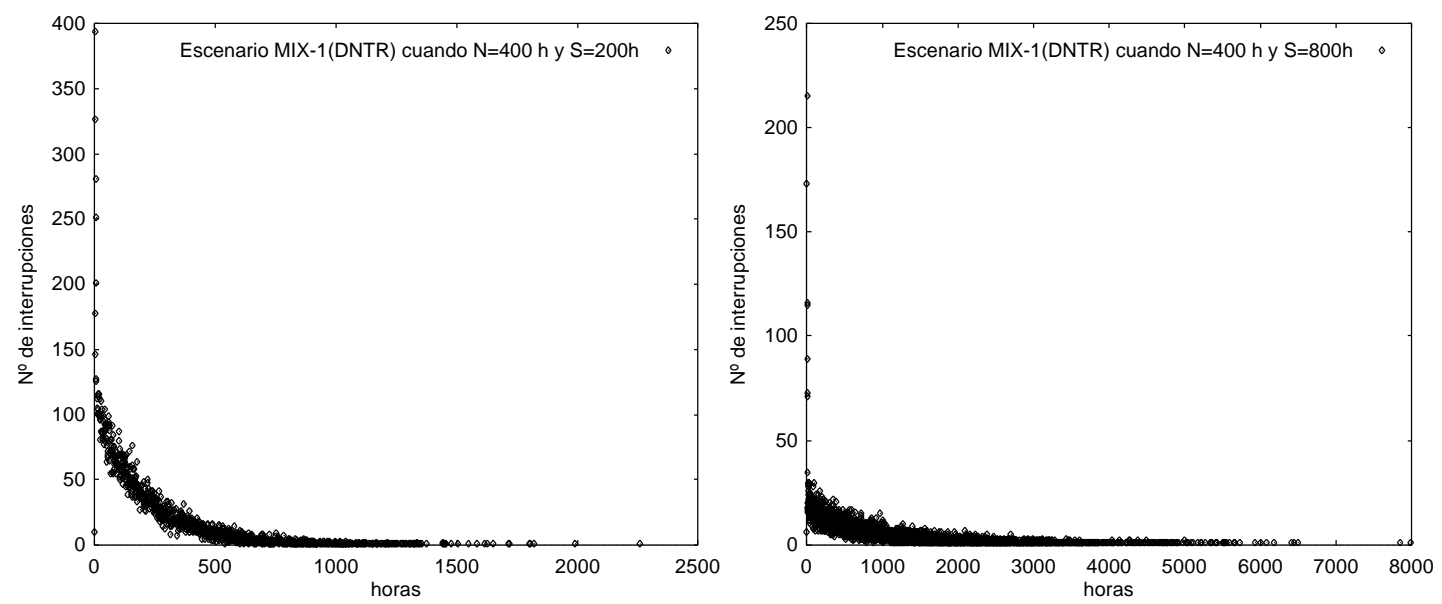

Fig.4.56.-Distribuciones del tiempo de indisponibilidad para el punto de carga número "58" de la red mixta cuando se asigna a $S$ valores de 200 y 800 horas. 

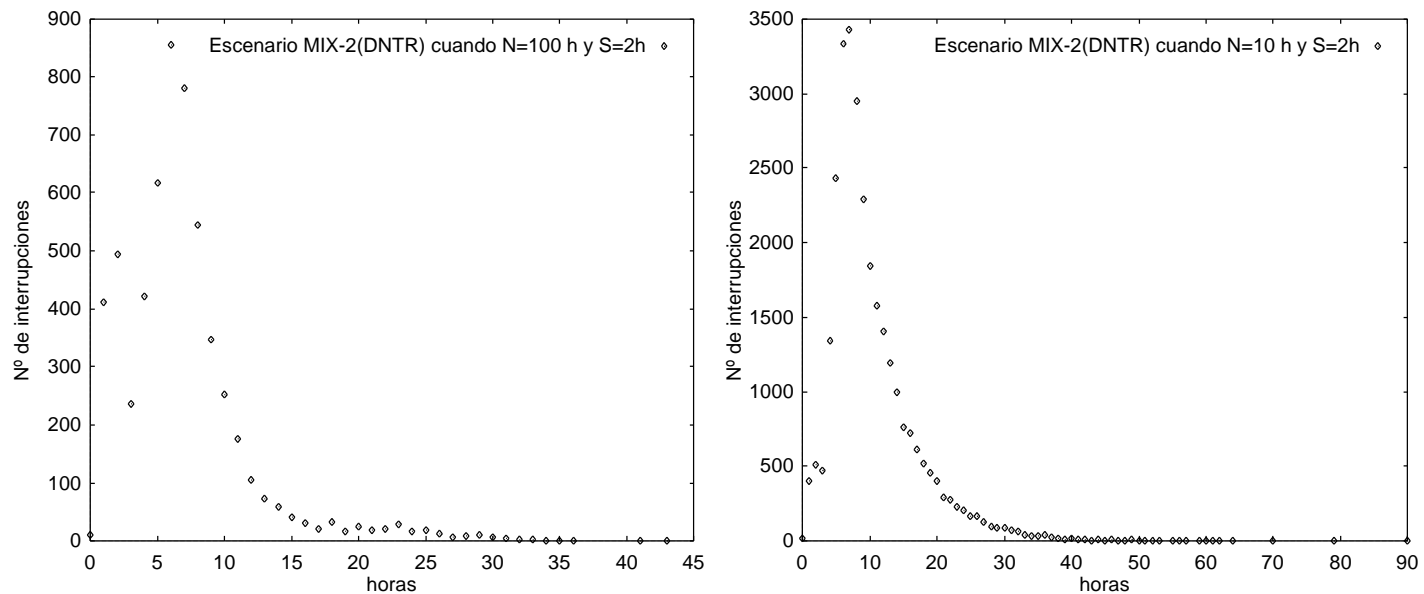

Fig.4.57.-Distribuciones del tiempo de indisponibilidad para el punto de carga número "58" de la red mixta cuando se asigna a $N$ valores de 100 y 10 horas.

\section{ASPECTOS A DESTACAR SOBRE LAS REDES MIXTAS}

Las conclusiones sobre la red mixta son idénticas a la obtenidas para la red aérea.

\subsection{SIMULACIÓN DEL COMPORTAMIENTO DE LA RED MEDIANTE LAS DISTRIBUCIONES ASOCIADAS A SUS PUNTOS DE CARGA}

Al principio de este capítulo señalábamos que era importante encontrar el tipo de distribución estadística que sigue el "Tiempo hasta la Interrupción" y el "Tiempo de Indisponibilidad" de la secuencia equivalente de ciclos de funcionamiento-fallo de cada punto de carga que componen una red.

Una de las dos razones que aducíamos para esta afirmación era que para obtener el ciclo de funcionamiento-fallo de cada punto de carga ya no necesitaríamos simular el funcionamiento de todos los elementos del punto de carga para luego sumarlos; bastaría con utilizar estas dos distribuciones asociadas a cada punto de carga como si de la simulación de un único elemento se tratase. Estas distribuciones son la síntesis, desde el punto de vista de la fiabilidad, de todos los elementos que componen los GMC de cada punto de carga.

Este modo de actuar incrementaría la velocidad de computo para posteriores simulaciones en las que buscásemos otros resultados derivados de los ciclos funcionamiento-fallo de los puntos de carga de la red, y asociados, por ejemplo, a los costos que los fallos de la red provocan en el consumidor o la compañía. Esta reducción en el tiempo de computo necesario para completar una simulación, podemos cuantificarla, para la red subterránea, en un 30\%, y, para la red aérea, en un $90 \%$. 
La automatización, mediante una aplicación informática, del proceso de identificación de la distribución estadística que siguen el "Tiempo hasta la Interrupción" y el "Tiempo de Indisponibilidad" de cada punto de carga en todos los escenarios, no es propósito de esta Tesis. Sin embargo, a modo ilustrativo se ha desarrollado este proceso para algunos escenarios, como se describe a continuación.

En las figuras 4.58 y 4.59 se muestran los resultados obtenidos para la red subterránea en el escenario SUB-1 (DETR). Se aprecia que la ley de las dos distribuciones obtenidas, tanto para el tiempo de indisponibilidad como para el tiempo hasta la interrupción, es la misma. Además, la concordancia cuantitativa, entre las distribuciones obtenidas por simulación de todos los elementos y las obtenidas mediante las distribuciones aociadas a cada punto de carga, también es notable.

El tiempo de cómputo de la indisponibilidad asociada a la red subterránea y a sus puntos carga, se redujo en un $30 \%$ para este escenario, figuras 4.58 y 4.59 .

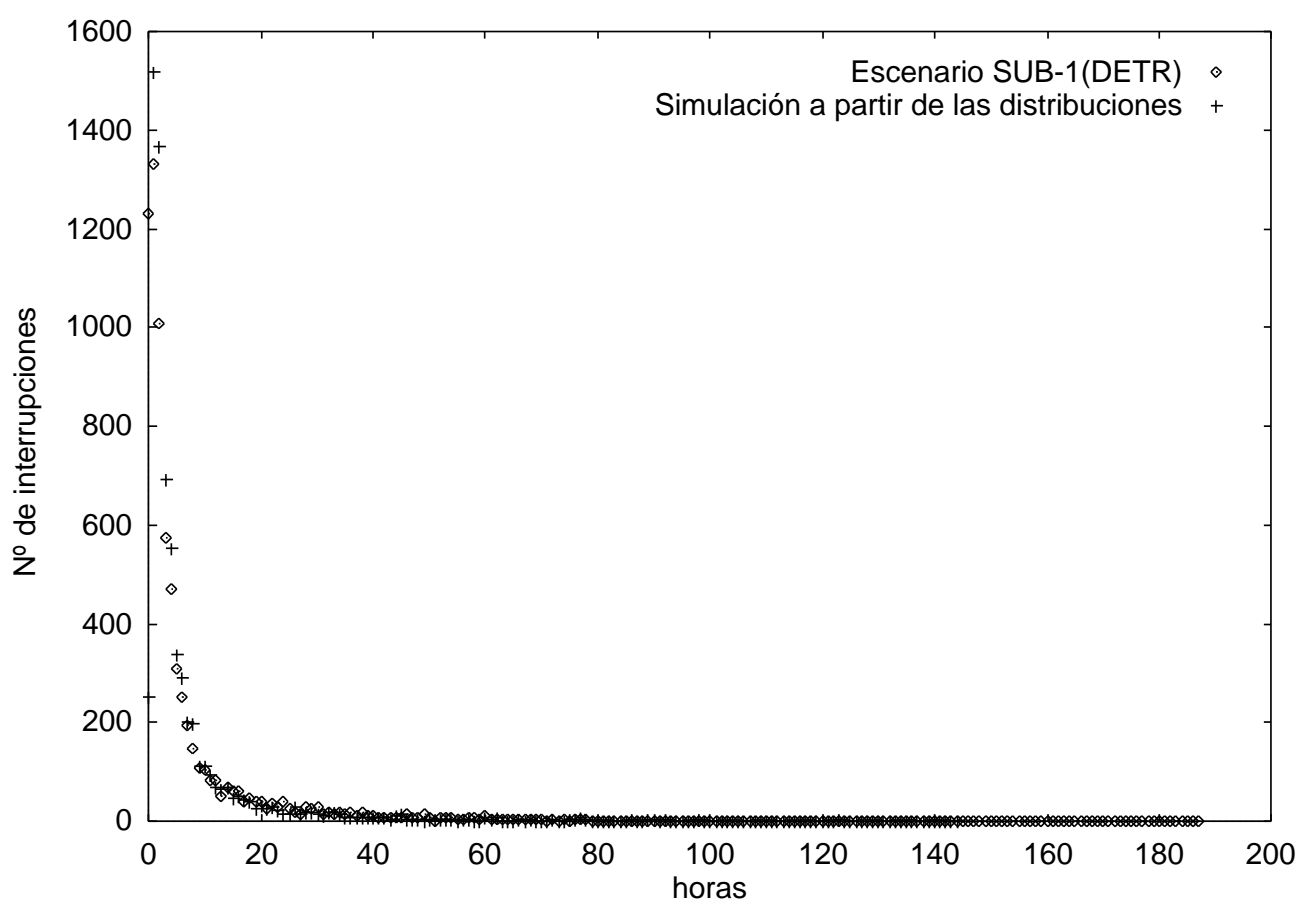

Fig.4.58.-Comparación entre las distribuciones del tiempo de indisponibilidad para el punto de carga número "10" de la red subterránea en el escenario SUB-1 (DETR) y cuando simulamos a partir de las distribuciones estadísticas obtenidas para ese punto de carga. 


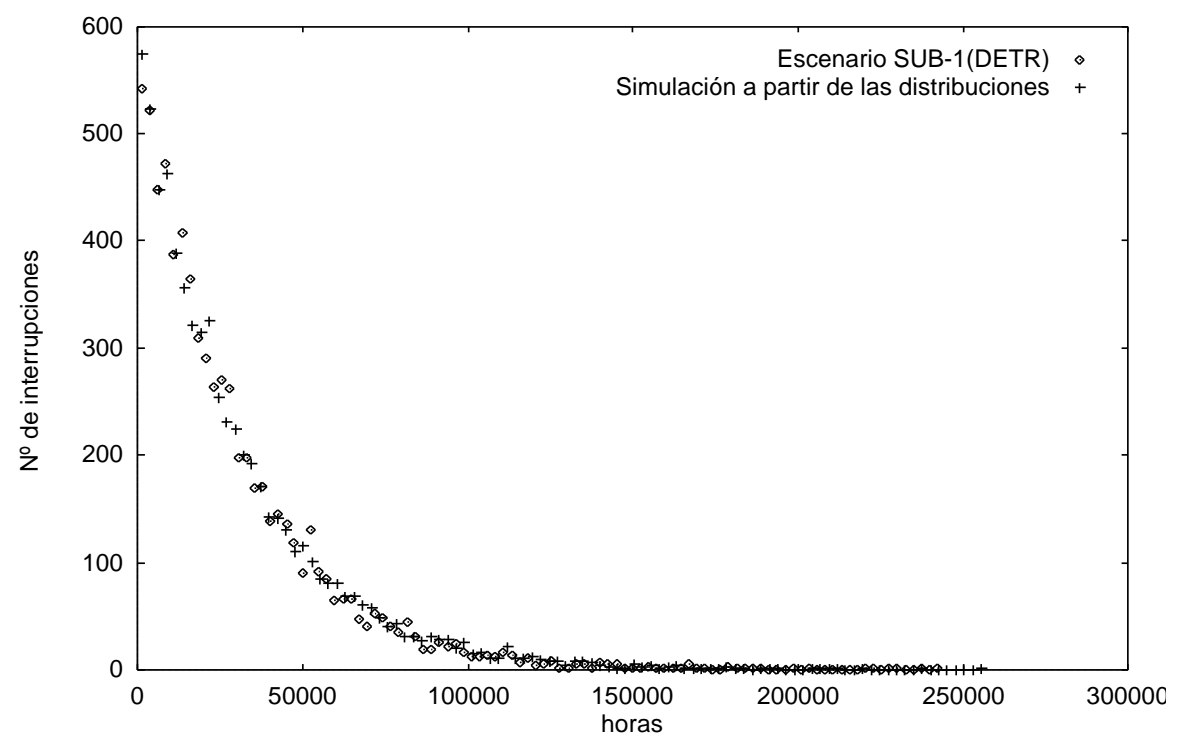

Fig.4.59.-Comparación entre las distribuciones del tiempo hasta la interrupción para el punto de carga número "10" de la red subterránea en el escenario SUB-1 (DETR) y cuando simulamos a partir de las distribuciones estadísticas obtenidas para ese punto de carga.

En las figuras 4.60 y 4.61 se muestran los resultados obtenidos para la red aérea en el escenario AER-1 (DETR) cuando $\mathrm{N}=400 \mathrm{~h}$ y $\mathrm{S}=2 \mathrm{~h}$. El resultado es similar al obtenido, anteriormente, con la red subterránea, ya que se aprecia que la ley de las dos distribuciones obtenidas, tanto para el tiempo de indisponibilidad como para el tiempo hasta la interrupción, es la misma. No obstante, existe una pequeña tendencia a aumentar el número de interrupciones en la distribución obtenida mediante síntesis. Esta tendencia a incrementar el número de interrupciones es un error corregible y debido a que el proceso de automatización necesita un ajuste más fino.

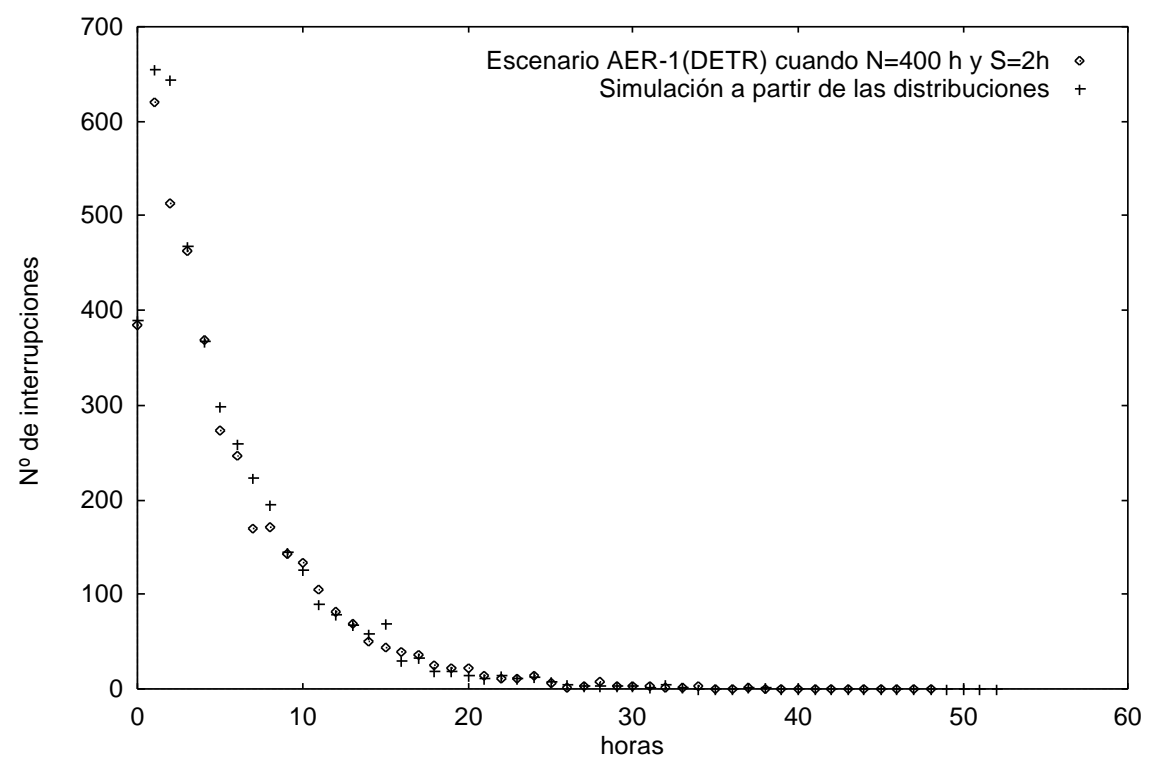

Fig.4.60.-Comparación entre las distribuciones del tiempo de indisponibilidad para el punto de carga número "10" de la red aérea en el escenario AER-1 (DETR) y cuando simulamos a partir de las distribuciones estadísticas obtenidas para ese punto de carga. 
El tiempo de cómputo de la indisponibilidad asociada a la red aérea y a sus puntos carga, se redujo en un $90 \%$ para este escenario, figuras 4.60 y 4.61 .

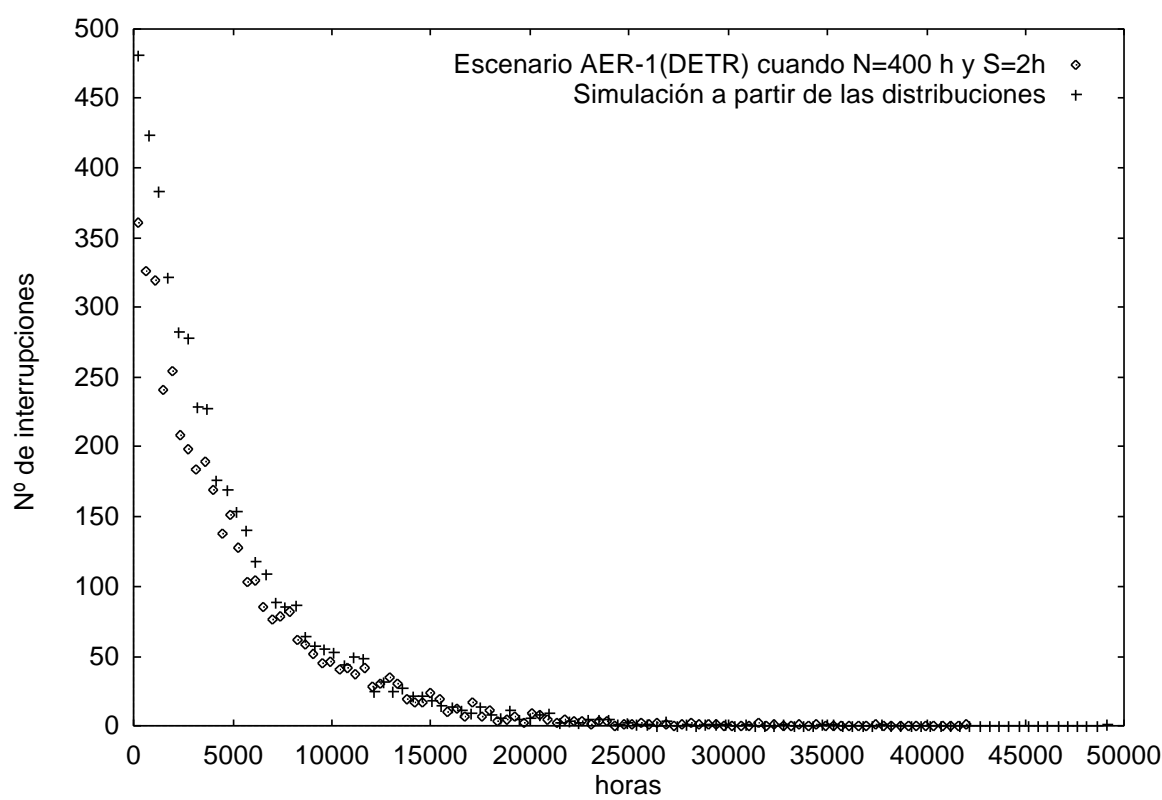

Fig.4.61.-Comparación entre las distribuciones del tiempo hasta la interrupción para el punto de carga número "10" de la red aérea en el escenario AER-1 (DETR) y cuando simulamos a partir de las distribuciones estadísticas obtenidas para ese punto de carga. 
CONCLUSIONES

\subsection{CONCLUSIONES}

Se ha alcanzado el objetivo de diseñar un procedimiento de simulación estocástica de eventos para diversas configuraciones de componentes en una Red de Distribución Eléctrica de Media Tensión. Este procedimiento de simulación ha sido implementado en una computadora digital.

Partiendo de los datos estimados de tasas de fallo y tiempos de reposición de servicio de los componentes de la red, se ha conseguido:

- Verificar o, en su caso, predecir los Índices de Calidad al uso en Sistemas de Distribución

- Obtener la distribución de frecuencias del "Tiempo hasta la Interrupción" y del "Tiempo de Reposición de Servicio" o "Tiempo de Indisponibilidad" de cada punto de carga del Sistema de Distribución de Energía Eléctrica en Media Tensión, como magnitudes debidamente parametrizadas. No existe, a este recpecto, ningún resultado publicado, ni tratamiento equivalente.

Dada la posibilidad que poseen los métodos de simulación para proporcionar técnicas de análisis más flexibles que los planteamientos denominados clásicos, esta Tesis ha dedicado una especial atención a los sistemas cuyos componentes se encuentran sometidos a ciclos de funcionamiento-reparación bajo condiciones atmosféricas variables, y en donde las duraciones correspondientes a los ciclos atmosféricos, no se hallan, necesariamente, distribuidos de forma exponencial. 
Se ha comprobado la influencia de distintos generadores de números aleatorios en la velocidad y grado de convergencia, a su valor teórico, de nuestro método de simulación, encontrando algunas diferencias entre los distintos generadores empleados. El proceso de selección final del generador más adecuado, requirió diversos tipos de pruebas, tanto estadísticas como prácticas, para analizar tanto la uniformidad como la aleatoriedad de los conjuntos de números generados.

Hasta ahora, no se había estudiado la dependencia que los índices de calidad al uso en Sistemas de Distribución tienen respecto de la ley de distribución del tiempo de reparación de los elementos que componen una red. En consecuencia, hemos analizado si, realmente, las aportaciones realizadas mediante la simulación modifican sustancialmente los resultados de las técnicas clásicas y, el grado de validez de las hipótesis simplificadoras empleadas por tales técnicas. Ello ha determinado que, además de la distribución exponencial, que es la empleada por las técnicas clásicas, se haya utilizado una distribución normal truncada, como aproximación más realista. Los resultados alcanzados señalan, en lo que a índices de calidad se refiere, variaciones poco significativas para los tipos de redes objeto de nuestro análisis.

Se ha encontrado además, que, las hipótesis simplificadoras que normalmente se hacen en la técnica basada en los procesos de Markov, son bastante razonables, excepto cuando, la tasa de fallo de algún elemento o la duración media de los ciclos de mal tiempo sean anormalmente elevadas. No obstante, nuestro proceso de simulación, se muestra superior ya que tiene en cuenta los fallos en modo común.

Se ha encontrado una dependencia lineal entre la disminución del tiempo medio de localización y desplazamiento y el aumento del valor de los índices de calidad de una red.

Se ha estudiado la influencia relativa en los índices de calidad resultantes, de las características de fiabilidad de distintos elementos que componen una red de distribución. Como conclusión fundamental hemos obtenido que, para mejorar la calidad de servicio de este tipo de redes, sería necesario realizar una inversión encaminada a disminuir la tasa de fallo y/o tiempo de reparación de los tramos aéreos, con prioridad a una actuación equivalente sobre cualquier otro elemento.

Para el tipo de redes objeto de esta Tesis, se han obtenido resultados de referencia de índices de calidad y de las distribuciones del "Tiempo hasta la Interrupción" y del "Tiempo de Indisponibilidad". Se han incorporado, para ello, diferentes condiciones meteorológicas y distintas características de fiabilidad de los elementos que componen las redes.

Resulta una aportación significativa, en esta Tesis, el haber encontrado, para las redes bajo análisis, el tipo de distribución estadística que siguen las distribuciones del "Tiempo hasta la Interrupción" y del "Tiempo de Indisponibilidad" de cada punto carga. La capacidad de ajustar por una distribución estadística conocida estas dos distribuciones, asociadas a cada punto carga de la red, nos facilita la búsqueda de 
otros índices relacionados con una distribución de probabilidad, como la probabilidad de que la duración de una interrupción sea menor que una de valor dado, o la probabilidad de que se llegue a un tiempo dado sin que el servicio se halla interrumpido.

Para las redes bajo análisis, en esta Tesis, se ha encontrado que puede ajustarse la distribución del "Tiempo hasta la Interrupción" de los puntos carga por una distribución exponencial.

El tratamiento ha permitido encontrar que, en lo que se refiere a la distribución del "Tiempo de Indisponibilidad" de los puntos carga de una red subterránea y cuando la hipótesis de distribución de los tiempos de reparación de los elementos de la red obedecen a una distribución exponencial, aquel puede ajustarse mediante una suma de funciones exponenciales o por una función de tipo exponencial. Cuando la hipótesis de distribución de los tiempos de reparación de los elementos de la red es una normal truncada, el tiempo de indisponibilidad puede ajustarse mediante una suma de distribuciones normales.

Igualmente resulta significativo destacar, que la distribución del "Tiempo de Indisponibilidad" de los puntos carga de una red aérea y/o mixta, cuando la hipótesis de distribución de los tiempos de reparación de los elementos de la red obedecen a una distribución exponencial, puede ajustarse mediante una distribución exponencial, una suma de dos exponenciales, o una distribución logarítmico-normal, dependiendo de las duraciones medias de los ciclos de buen y mal tiempo. Cuando la hipótesis de distribución de los tiempos de reparación sigue una distribución normal truncada, la distribución del "Tiempo de Indisponibilidad" puede ajustarse mediante una suma de distribuciones normales, o por una distribución logarítmico-normal, dependiendo, también, de las duraciones medias de los ciclos de buen y mal tiempo.

Igualmente significativa resulta la aportación relativa a la medida de los límites superior e inferior de las distribuciones del "Tiempo hasta la Interrupción" de los puntos carga para redes de Distribución, constatando su estrecha dependencia con las tasas de fallo de los elementos que las componen y con la duracion media de los ciclos de buen y mal tiempo.

De forma paralela se ha medido el límite superior de las distribuciones del "Tiempo de Indisponibilidad", quedando patente su dependencia con los tiempos medios de reparación, y con el tipo de distribución elegida para estos y para la duración media de los ciclos de mal tiempo. Si se utiliza una normal truncada para la distribución del tiempo medio de repación, se ha encontrado que disminuye el límite superior de la distribución del "Tiempo de Indisponibilidad". En el caso de una red subterránea, el factor de disminución puede alcanzar hasta un valor 5.

El acotado de la duración de las interrupciones y de la continuidad de servicio, son nuevos índices que permiten un mayor conocimiento de la calidad del servicio 
que ofrece una Compañía Eléctrica en una red de Distribución, y como tal se proponen.

Ha quedado puesto de manifiesto, que asociar la utilización de las distribuciones del "Tiempo hasta la Interrupción" y del "Tiempo de Indisponibilidad" a cada punto carga, para posteriores simulaciones, como si de la simulación de un único elemento se tratase, incrementa, sensiblemente, la velocidad de cálculo. El tiempo de cálculo se ha reducido hasta un $30 \%$ en el caso de las redes subterráneas y en un $90 \%$ en el caso de las redes aéreas. Esta técnica permitiría agilizar la búsqueda de otros resultados derivados de los ciclos funcionamiento-fallo de puntos carga de la red, como por ejemplo la repercusión de los costos de los fallos de red en el consumidor o la compañía.

Valladolid, 7 de octubre de 1996

Fernando A. Frechoso Escudero 
(M_CAL-96): Iberdrola, Uitesa, Dept. Ingeniería Eléctrica de la Universidad de Valladolid. Memoria del Proyecto PIE : CALSER (Análisis Predictivo de la Fiabilidad en Redes de Distribución Para la mejora de la Calidad de Servicio), Actividad C, Enero1996.

(A_RIE-FRE-96): M.V. Riesco, F.A. Frechoso, P.C. Alvarez, A. Gordaliza. Validating Theoretical Hypothesis on Access Times and Repair Times Statistical Distributions for Electric Power Distribution Network, Melecon 96 Proceedings, Vol. 2, p765-767.

(A_SAN-BIL-95): A. Sankarakrishnan, R. Billinton. Sequential Monte Carlo Simulation for Composite Power System Reliability Analysis with Time Varying Loads, IEEE Trans. on PS, Vol. 10, No 3, August 1995, p1540-1545.

(A_BHU-ALL-94): M.R. Bhuiyan, R.N. Allan. Inclusion of Weather Effects in Composite System Reliability Evaluation Using Sequential Simulation, IEE Proc.-Gener. Transm. Distrib., Vol. 141, No 6, November 1994, p575-584.

(A_FRE-ALA-94): F.A. Frechoso Escudero, J.L. del Alamo y del Sarmiento, J. Devesa Fernández. La generación de números aleatorios y su influencia en la simulación estocástica de eventos, Libro de comunicaciones del Congreso de Recursos y Tecnologías Electro-Energéticas, Orense (España), octubre de 1994.

(A_BILL-CHA-94): R. Billinton, E. Chan, G. Wacker. Probability Distribution Approach to Describe Costs Due to Electric Supply Interruptions, IEE Proc.-Gener. Transm. Distrib., Vol. 141, No 6, November 1994, p594-598.

(A_BIL-LI-93): R. Billinton, W. Li. A System State Transition Sampling Method for Composite System Reliability Evaluation, IEEE Trans. on PS, Vol. 8, No 3, August 1993, p761-766.

(A_CHO-TAY-93): M. Chow, L.S. Taylor. A Novel Approach for Distribution Fault Analysis, IEEE Trans. on PD, Vol. 8, No 4, October 1993, p1882-1888.

(A_PER-PIN-92): M.V.F.. Pereira, L.M.V.G.. Pinto. A New Computational Tool for Composite Reliability Evaluation, IEEE Trans. on PS, Vol. 7, No 1, February 1992, p258-264. 
(A_PER-PIN-92 bis): M.V.F.. Pereira, M.E.P. Maceira, G.C. Oliveira, L.M.V.G.. Pinto. Combining Analytical Models and Monte-Carlo Techniques in Probabilistic Power System Analysis, IEEE Trans. on PS, Vol. 7, No 1, February 1992, p265-272.

(A_UBE-ALL-92): J.R. Ubeda, R.N. Allan. Sequential Simulation to Composite System Reliability Evaluation, IEE Proc.-C, Vol. 139, No 2, March 1992, p81-86.

(L_BIL-ALL-92): R. Billinton, R.N. Allan. Reliability Evaluation of Engineering Systems, Plenum Press, 1992.

(L_VILL-92): A. Villemeur. Reliability, Availability, Maintainability and Safety Assessment, John Wiley \& Sons, 1992.

(L_MAT-92): Drago Matko, Rihard Karba, Borut Zupancic. Simulation and Modelling of Continuous Systems, Prentice Hall, 1992.

(L_PRE-TEU-92): W.H. Press, S.A. Teukolsky, etal. Numerical Recipes in C, Cambridge University Press, 1992.

(A_BIL-WEN-91): R. Billinton, L.. Wenyuan. Hybrid Approach for Reliability Evaluation of Composite Generation and Transmission Systems Using Monte-Carlo Simulation and Enumeration Technique, IEE Proc.-C, Vol. 138, No 3, May 1991, p233-241.

(A_BIL-WEN-91 bis): R. Billinton, L.. Wenyuan. A Novel Method for Incorporating Weather Effects in Composite System Adequacy Evaluation, IEEE Trans. on PS, Vol. 6, No 3, August 1991, p11541160 .

(L_IEEE-91): IEEE. IEEE Recommended Practice for the Design of Reliable Industrial and Commercial Power Systems, IEEE Inc., 1991.

(L_CEL-91): François E. Cellier. Continuous System Modelling, Springer-Verlag, 1991.

(A_ROL-ALV-91): C. Roldán, C. Álvarez, E. Quiles. Evaluación Probabilística de la Fiabilidad en Redes de Distribución Reconfigurables. Comunicación interna.

(A_WAN-89): L. Wang. Approximate Confidence Bounds on Monte Carlo Simulation Results for Energy Production, IEEE Trans. on PS, Vol. 4, No 1, February 1989, p69-74.

(A_OLI-PER-89): G.C. Oliveira, M.V.F.. Pereira, S.H.F.. Cunha. A Technique for Reducing Computational Effort in Monte-Carlo based Composite Reliability Evaluation, IEEE Trans. on PS, Vol. 4, No 4, October 1989, p1309-1315.

(A_LEC-88): P. L'Ecuyer.Communications of th ACM, Vol 31,1988, p742-774.

(L_DAG-88): J. Dagpunar. Principles of Random Variate Generation, Oxford University Press, 1988.

(A_PAR-MIL-88): S.K. Park, K.W. Miller.Communications of th ACM, Vol 31,1988, p1192-1201.

(A_PAT-BLA-88): A.D. Patton, J.H. Blackstone, N.J. Balu. A Monte Carlo Simulation Approach to the Reliability Modeling of Generating Systems Recognizing Operating Considerations, IEEE Trans. on PS, Vol. 3, No 3, August 1988, p1174-1180. 
(A_CUN-PER-85): S.H.F.. Cunha, M.V.F.. Pereira, L.M.V.G. Pinto, G.C. Oliveira. Composite Generation and Transmission System Reliability Evaluation in Large Hydroelectric Systems, IEEE Trans. on PAS, Vol. 104, No 10, October 1985, p2657-2663.

(A_SAL-BIL-85): L. Salvaderi, R. Billinton. A Comparison between Two Fundamentally Different Approaches to Composite System Reliability Evaluation, IEEE Trans. on PAS, Vol. 104, No 12, December 1985, p3486-3492.

(A_MAR-ROB-83): K. Marse, S.D. Roberts. Implementing a Portable Fortran Uniform $(0,1)$ Generator, Simulation, Vol. 41, 1983, p135-139.

(L_BIL-ALL-83): R. Billinton, R.N. Allan. Reliability Evaluation of Engineering Systems, Concepts and Techniques, Pitman Advanced Publishing Programs, 1983.

(L_HIL-LIE-82): F. Hillier, G.J.Lieberman. Introducción a la Investigación de Operaciones, McGraw-Hill $1^{a}$ edición en español, 1982.

(L_COS-82): R. Coss Bu. Simulación un enfoque práctico, Limusa S.A., 1982.

(L_RUB-81): R. Y. Rubinstein. Simulation and the Monte Carlo Method, John Wiley \& Sons, 1981.

(L_KNU-81): D.E. Knuth. The Art of Computer Programming: Seminumerical Algorithms, Vol. 2, Addison-Wesley, 1981.

(A_KOV-BIL-79): D.O. Koval, R. Billinton. Evaluation of Distribution Circuit Reliability, IEEE Trans. on PAS, Vol. 98, No 2, March/April 1979, p509-517.

(A_ALL-DIA-79): R.N. Allan, E.N. Dialynas, I.R. Homer. Modelling and Evaluating the Reliability of Distribution Systems, IEEE Trans. on PAS, Vol. 98, No 6, November/December 1979, p21812189.

(A_SCH-79): L. Schrage. ACM Transactions on Mathematical Software, Vol 5, 1979, p132-138.

(L_ARN-78): G. Arnáiz Vellando, Introducción a la Estadística Teórica, Lex Nova, 1978.

(L_SOB-76): I.M. Sóbol. Método de Montecarlo, Mir, 1976.

(A_BAY-DUR-76):.C. Bays, S.D. Durham. ACM Transactions on Mathematical Software, Vol 2, 1976, p59-64.

(A_NOF-PAR-75): P.L. Noferi, L. Paris, L. Salvaderi. Proccedings 1975 Annual Reliability and Maintainability Symposium, January 1975, p449-459.

(A_BIL-GRO-75): R. Billinton, M.S. Grover. Reliability Assessment of Transmission and Distribution Schemes, IEEE Trans. on PAS, Vol. 94, No 3, May/June 1975, p724-732.

(L_SHA-75): R. E. Shannon. Systems Simulation: The Art and Science, Prentice-Hall, 1975.

(L_BIL-RIN-73): R. Billinton, R.J. Ringlee, A.J. Wood. Power-System Reliability Calculations, The MIT Press, 1973.

(A_RIN-GOO-70): R.J. Ringlee, S.D. Goode. On Procedures for Reliability Evaluations of Transmission Systems, IEEE Trans. on PAS, Vol. 80, No 4, April 1970, p527-536. 
(A_BIL-BOL-68): R. Billinton, K.E. Bollinger. Transmission System Reliability Evaluation Using Markov Processes, IEEE Trans. on PAS, Vol. 87, No 2, February 1968, p538-547.

(L_NAY-66): T.J. Naylor, J.L. Balintfy, D.S. Burdick, and K. Chu, Computer Simulation Techniques, Wiley, 1966.

(A_GAV-MON-64): D.P. Gaver, F.E. Montmeat, A.D. Patton. Power System Reliability. 1-Measures of Reliability and Methods of Calculation, IEEE Trans. on PAS, July 1964, p727-737.

(A_TOD-64): Z.G. Todd. A Probability Method for Transmision and Distribution Outage Calculations, IEEE Trans. on PAS, July 1964, p695-701.

(A_MAR-62): G. Marsaglia. Boeing Scientific Res. Lab. report, D1-82-0203, 1962.

(L_COX-62): D.R. Cox, Renewal Theory, Methuen \& Co. Ltd, 1962.

(A_BOX-MUL-58): G.E.P. Box, M.E. Muller. A Note on the Generation of Random Normal Deviates, Annals Math. Statist., Vol 29, 1958, p610-611.

(A_LEH-51):.D.H. Lehmer. Proccedings 2nd Symposium on Large-Scale Digital Calculating Machinery, Harvard University Press, 1951, p141-146. 
COMPONENTES CONSIDERADOS

\section{ANEXO 1: COMPONENTES CONSIDERADOS}

Definiremos en este anexo:

- Tipos y subtipos de componentes incorporables a los análisis por simulación.

- Selección de los que, específicamente, se integran en las redes de ensayo utilizadas.

- Breve descripción de todos los elementos que componen las redes de ensayo.

Se tabulan en la Tabla A1-1, los diferentes tipos y subtipos de componentes que susceptibles de analizarse, mediante la aplicación informática desarrollada en esta Tesis.

Todos los elementos que se especifican en la Tabla A1-1 no serán empleados para construir las redes de ensayo; por sencillez se utilizará una selección de estos. Específicamente, los resultados que se aportan en esta Tesis, se basan en considerar a los siguientes elementos (ver Tabla A1-2) como constituyentes, en su caso, de cada Red de Distribución empleada como ejemplo.

\begin{tabular}{|c|c|c|}
\hline Tipo de componente & Subtipo & Nombre \\
\hline
\end{tabular}




\begin{tabular}{|c|c|c|}
\hline Cable subterráneo & $\begin{array}{l}\text { Cable unipolar de aislamiento seco de cobre } \\
\text { Cable unipolar de aislamiento seco de aluminio } \\
\text { Cable unipolar de papel impregnado de cobre } \\
\text { Cable unipolar de papel impregnado de aluminio } \\
\text { Cable tripolar de aislamiento seco de cobre } \\
\text { Cable tripolar de aislamiento seco de aluminio } \\
\text { Cable tripolar de papel impregnado de cobre } \\
\text { Cable tripolar de papel impregnado de aluminio }\end{array}$ & $\begin{array}{l}\text { CS0 } \\
\text { CS1 } \\
\text { CS3 } \\
\text { CS4 } \\
\text { CS6 } \\
\text { CS7 } \\
\text { CS8 } \\
\text { CS9 }\end{array}$ \\
\hline Línea aérea & $\begin{array}{l}\text { con conductor LA56, UNE21018 } \\
\text { con conductor LA78, UNE21018 } \\
\text { con conductor LA110, UNE21018 }\end{array}$ & $\begin{array}{l}\text { LA0 } \\
\text { LA1 } \\
\text { LA2 }\end{array}$ \\
\hline $\begin{array}{l}\text { Interruptor automático de } \\
\text { cabecera de línea }\end{array}$ & $\begin{array}{l}\text { de interior extraíble } \\
\text { de interior fijo } \\
\text { de intemperie fijo } \\
\end{array}$ & $\begin{array}{l}\text { IA0 } \\
\text { IA4 } \\
\text { IA7 } \\
\end{array}$ \\
\hline Autoseccionador & $\begin{array}{l}\text { de intemperie manual } \\
\text { de intemperie telemandado }\end{array}$ & $\begin{array}{l}\text { AS0 } \\
\text { AS7 }\end{array}$ \\
\hline Ruptofusible & & RF0 \\
\hline Seccionador-Fusible & & XS0 \\
\hline Seccionador & $\begin{array}{l}\text { de interior manual } \\
\text { de interior telemandado } \\
\text { de intemperie manual } \\
\text { de intemperie telemandado }\end{array}$ & $\begin{array}{l}\text { SV0 } \\
\text { SV3 } \\
\text { SV5 } \\
\text { SV7 }\end{array}$ \\
\hline Interruptor-Seccionador & $\begin{array}{l}\text { de interior manual } \\
\text { de interior telemandado } \\
\text { de intemperie manual } \\
\text { de intemperie telemandado }\end{array}$ & $\begin{array}{l}\text { IS0 } \\
\text { IS3 } \\
\text { IS5 } \\
\text { IS7 } \\
\end{array}$ \\
\hline Fusible & & FU0 \\
\hline Reconectador & $\begin{array}{l}\text { de intemperie manual } \\
\text { de intemperie telemandado }\end{array}$ & $\begin{array}{l}\mathrm{RC} 0 \\
\mathrm{RC} 5\end{array}$ \\
\hline Transformador & $\begin{array}{l}\text { de interior } \\
\text { de intemperie }\end{array}$ & $\begin{array}{l}\text { TF0 } \\
\text { TF5 } \\
\end{array}$ \\
\hline $\begin{array}{l}\text { Salida de Subestación de } \\
\text { Maniobra y/o Reparto }\end{array}$ & & SA0 \\
\hline
\end{tabular}

Tabla A1-1. Tipos y subtipos de componentes incorporables a los análisis por simulación.

\begin{tabular}{|c|c|c|c|}
\hline Nombre & Red aérea & Red subterránea & Red mixta \\
\hline CS0 & & $\mathrm{X}$ & $\mathrm{X}$ \\
\hline LA1 & $\mathrm{X}$ & & $\mathrm{X}$ \\
\hline IA0 & $\mathrm{X}$ & $\mathrm{X}$ & $\mathrm{X}$ \\
IA4 & & & $\mathrm{X}$ \\
\hline AS0 & $\mathrm{X}$ & $\mathrm{X}$ & $\mathrm{X}$ \\
\hline RF0 & & $\mathrm{X}$ & $\mathrm{X}$ \\
\hline XS0 & $\mathrm{X}$ & & $\mathrm{X}$ \\
\hline SV0 & $\mathrm{X}$ & $\mathrm{X}$ & $\mathrm{X}$ \\
SV5 & & & $\mathrm{X}$ \\
\hline IS0 & $\mathrm{X}$ & $\mathrm{X}$ & $\mathrm{X}$ \\
\hline FU0 & $\mathrm{X}$ & $\mathrm{X}$ & $\mathrm{X}$ \\
\hline TF0 & $\mathrm{X}$ & & \\
TF5 & & & \\
\hline SA0 & & & \\
\hline
\end{tabular}

Tabla A1-2. Elementos que, específicamente, se integran en las Redes de Ensayo utilizadas.

Brevemente, se describen los tipos de elementos considerados:

\section{Lineas Aéreas}


Se conceptúan como un todo que incluye el conductor, los apoyos, aisladores, grapas, etc., así como otros elementos auxiliares (señalizadores de paso de falta, pararrayos, etc.). Se ha considerado al conductor, normalmente, desnudo y constituido de cobre o aluminio con alma de acero. Su tasa de fiabilidad se define por $\mathrm{Km}$. de longitud. Al ser un elemento muy abundante y el hecho de encontrarse expuesto a las inclemencias meteorológicas, le otorga una gran relevancia en los análisis de la fiabilidad total del sistema.

\section{Cables Subterráneos}

Comprenden al conductor y las botellas terminales. Al no encontrarse expuesto a situaciones atmosféricas adversas, excepto las botellas terminales intemperie, su fiabilidad es mayor que la de las líneas aéreas. Existen varios parámetros que los caracterizan: el material conductor (aluminio o cobre), la disposición de los conductores (unipolar o tripolar), y el tipo de aislamiento (seco o de papel impregnado).

\section{Interruptor Automático}

Como elemento capaz de establecer, mantener, o interrumpir la intensidad de la corriente de servicio o de interrumpir automáticamente o establecer, en condiciones predeterminadas, intensidades de corriente anormalmente elevadas, tales como las corrientes de cortocircuito. Pueden ser de interior (fijos o extraibles) o de intemperie. Se encuentran, bien en las Subestaciones de Maniobra y/o Reparto, o bien en los Centros de maniobra y Reparto.

\section{Autoseccionador (Seccionalizador)}

Como elemento que abre un circuito automáticamente en condiciones predeterminadas, cuando el circuito está sin tensión. La apertura se produce cuando detecta un número discreto de veces una sobreintensidad en una parte de la red (o un cero de tensión provocado por la apertura de un reconectador situado aguas arriba). $\mathrm{Su}$ aportación principal es que permite distinguir las faltas transitorias de las permanentes. Ejerce eventualmente funciones de maniobra. El accionamiento para maniobra es casi siempre manual aunque en ocasiones puede ser, además, telemandado. Es de intemperie, y va situado en cabecera de derivaciones importantes del alimentador principal, en cabecera de algunas derivaciones secundarias, o incluso en el alimentador principal, a distancia de los reconectadores.

\section{Ruptofusible}

Interruptor que, en su posición de apertura, satisface las condiciones de aislamiento especificadas para un seccionador y en el que uno o más polos poseen un fusible en serie en un aparato combinado, permitiendo de esta forma actuar de modo automático. Su función también es la maniobra. Como tal, su accionamiento es exclusivamente local. Van instalados siempre en interior, en las celdas de protección del trafos de interior.

\section{Seccionador-Fusible}


Es un aparato mecánico en el que el contacto móvil está formado por un elemento recambiable. Se denomina también cortacircuito fusible o fusible de expulsión. Como elemento de maniobra, su accionamiento es exclusivamente manual. Es un elemento de intemperie y constituye una protección en trafos de intemperie, y en cabeceras de lineas de clientes, así como en alguna derivaciones poco importantes.

\section{Seccionador}

Es un dispositivo mecánico de conexión que, por razones de seguridad, en posición de abierto, asegura una distancia de seccionamiento que satisface unas condiciones especificadas. Es capaz de abrir o cerrar el circuito cuando la corriente a interrumpir es despreciable, o bien cuando no se produce cambio significativo de tensión en los bornes de cada uno de los polos del seccionador. Es capaz de soportar corrientes nominales de paso así como corrientes de cortocircuito durante un tiempo especificado. El accionamiento puede ser telemandado o manual. Se instalan, tanto en interior como en intemperie. Van ubicados en posiciones intermedias de lineas aéreas, en algunas derivaciones, o como elementos constituyentes de celdas de maniobra.

\section{Interruptor-Seccionador}

Interruptor que, en posición de apertura, satisface las condiciones de aislamiento especificadas para un seccionador. Se denomina también, impropiamente, Seccionador en Carga. El mando puede ser manual o telemandado. Se instalan tanto en interior, como en intemperie en Subestaciones de Maniobra y/o Reparto o en posiciones intermedias de lineas aéreas, respectivamente.

\section{Fusible}

Elemento que actúa por fusión, dejando abierta, al menos, una fase de un circuito. Destinado a proteger, por fusión, una instalación o parte de ella contra sobreintensidades. Poseen alto poder de corte. Se sitúan tanto en interior como en intemperie, sirviendo como protección a los transformadores.

\section{Reconectador}

Interruptor automático de intemperie situado en zonas intermedias de las líneas en lugar de en la cabecera de las mismas. Usualmente asociado a seccionalizadores, que permiten el aislamiento definitivo de la falta tras un número predeterminado de reenganches efectuados mediante el propio reconectador.

\section{Transformador de Potencia}

Máquina estática, trifásica, con dos o más devanados por fase, destinada a modificar el nivel de tensión de la energía eléctrica. 


\section{PARAMETROS DE FIABILIDAD DE LOS COMPONENTES}

\section{ANEXO 2: PARAMETROS DE FIABILIDAD DE LOS COMPONENTES}

$\mathrm{Al}$ objeto de poder estimar tanto el número de incidencias que se produce en un punto de carga como el tiempo acumulado de indisponibilidad en el mismo, y dado que estas incidencias en la red tienen su origen en las indisponibilidades surgidas en sus componentes, es necesario conocer los parámetros de fiabilidad individual de los mismos. Estos parámetros se refieren tanto al número medio de fallos permanentes esperado en cada tipo de componente como a los tiempos medios de duración de la indisponibilidad en los mismos.

Definiremos en este anexo:

- Parámetros asociados con modelos de fiabilidad individuales.

- Nomenclatura para los modos de fallo y reposición que se consideran en esta Tesis.

- Datos numéricos de los parámetros de fiabilidad de todos los componentes incorporables a los análisis por simulación. 
Los parámetros asociados con los modelos de fiabilidad individuales de los componentes de las redes de Media Tensión, que utilizaremos en esta Tesis, son los que se reflejan en la Tabla A2-1.

\begin{tabular}{|c|c|}
\hline Componente & Parámetros de Fiabilidad \\
\hline Lineas Aéreas & $\begin{array}{l}\text { Tasa Media de Fallo: } \frac{\mathrm{N}^{\circ} \text { de averías por año }}{\mathrm{N}^{\circ} \text { de Km de línea }} \\
\text { Tiempo Medio de Reparación } \\
\text { Tiempo Medio de maniobra/accionamiento }\end{array}$ \\
\hline Cables Subterráneos & $\begin{array}{l}\text { Tasa Media de Fallo: } \frac{\mathrm{N}^{\circ} \text { de averías por año }}{\mathrm{N}^{\circ} \text { de Km de cable }} \\
\text { Tiempo Medio de Reparación } \\
\text { Tiempo Medio de maniobra/accionamiento }\end{array}$ \\
\hline $\begin{array}{l}\text { Elementos de Maniobra y/o Protección: } \\
\text { - Interruptor Automático } \\
\text { - Reconectador } \\
\text { - Autoseccionador } \\
\text { - Seccionador-Fusible } \\
\text { - Rupto-Fusible } \\
\text { - Fusible } \\
\text { - Seccionador } \\
\text { - Interruptor-Seccionador }\end{array}$ & $\begin{array}{l}\text { Tasa Media de fallo: } \frac{\mathrm{N}^{\circ} \text { de averías por año }}{\mathrm{N}^{\circ} \text { de componentes funcionando }} \\
\text { Tiempo Medio de Reparación } \\
\text { Tiempo Medio de pseudosubstitución } \\
\text { Tiempo Medio de maniobra/accionamiento }\end{array}$ \\
\hline Transformador Intemperie & $\begin{array}{l}\text { Tasa Media de Fallo: } \frac{\mathrm{N}^{\circ} \text { de averías por año }}{\mathrm{N}^{\circ} \text { de componentes funcionando }} \\
\text { Tiempo Medio de Reparación }\end{array}$ \\
\hline Transformador Interior & $\begin{array}{l}\text { Tasa Media de fallo: } \frac{\mathrm{N}^{\circ} \text { de averías por año }}{\mathrm{N}^{\circ} \text { de componentes funcionando }} \\
\text { Tiempo Medio de Reparación }\end{array}$ \\
\hline
\end{tabular}

Tabla A2-1. Parámetros asociados con modelos de fiabilidad individuales.

La terminología utilizada en la Tabla A2-1, se utiliza con el siguiente significado (veáse también el apartado 2.4):

- Tiempo Medio de Reparación: es el tiempo medio, en horas, de duración de cada operación de reparación a las que deba ser sometido un elemento en estado de avería para conseguir pasar a su estado de funcionamiento. Dentro de las actuaciones de reparación se contemplará también la sustitución del componente por otro de similares características y que desempeñe las mismas funciones. 
- Tiempo Medio de Pseudosustitución: es el tiempo medio de duración de cada operación de sustitución de un elemento en estado de avería por otro que no realice exactamente la misma función, pero que permita reanudar el servicio durante un tiempo determinado.

- Tiempo Medio de Maniobra/Accionamiento: es el tiempo medio de duración de las acciones efectuadas para que un elemento pase de su posición de abierto a cerrado o viceversa.

La nomenclatura para los modos de fallo y de reposición de servicio considerados es la que se recoge en la Tabla A2-2:

\begin{tabular}{|c|c|c|}
\hline \multirow{2}{*}{ Modos de fallo } & \multicolumn{2}{|c|}{ Tasas de fallo (fallos/año) (*) } \\
\cline { 2 - 3 } & tiempo normal & mal tiempo \\
\hline \multirow{2}{*}{ Permanente (Interrupción mayor a 1 minuto ) } & $\lambda_{\mathrm{j}} \mathrm{P}$ & $\mathrm{w}_{\mathrm{j}} \mathrm{P}$ \\
\hline \multirow{3}{*}{ Modos de reposición } & \multicolumn{2}{|c|}{ Duración de reposición (horas) } \\
\cline { 2 - 3 } & tiempo normal & mal tiempo \\
\hline Reparación/sustitución & $\mathrm{r}_{\mathrm{j}} \mathrm{R}$ & $\rho_{\mathrm{j}}^{\mathrm{R}}$ \\
Maniobra/accionamiento & $\mathrm{r}_{\mathrm{j}} \mathrm{A}$ & $\rho_{\mathrm{j}}^{\mathrm{A}}$ \\
Pseudosustitución & $\mathrm{r}_{\mathrm{j}}^{\mathrm{S}}$ & $\rho_{\mathrm{j}}^{\mathrm{S}}$ \\
\hline
\end{tabular}

(*) Las tasas de fallo se expresan en: fallos / Km·año) para Líneas Aéreas y Cables Subterráneos fallos / año para el resto de componentes.

Tabla A2-2. Nomenclatura para modos de fallo y reposición de servicio considerados. 
Los datos de fiabilidad básicos de los componentes susceptibles de analizarse mediante la aplicación informática desarrollada en esta Tesis, son los que se muestran en Tabla A2-3.

\begin{tabular}{|c|c|c|c|c|c|c|c|c|}
\hline \multirow[b]{3}{*}{ Nombre } & \multirow{2}{*}{\multicolumn{2}{|c|}{$\begin{array}{c}\text { Tasas de fallo }(\lambda=\text { buen } \\
\text { tiempo, } w=\text { mal tiempo) }\end{array}$}} & \multicolumn{6}{|c|}{$\begin{array}{c}\text { Duración de la reposición } \\
\text { ( } \mathrm{r}=\text { buen tiempo, } \rho=\text { mal tiempo) }\end{array}$} \\
\hline & & & \multicolumn{2}{|c|}{ Reparac./sustitución } & \multicolumn{2}{|c|}{ Maniobra/acciona. } & \multicolumn{2}{|c|}{ Pseudosustitución } \\
\hline & $\lambda^{\mathrm{P}}$ & ${ }^{P}{ }^{P}$ & $\mathrm{r}^{\mathrm{R}}$ & $\rho^{R}$ & $r^{\mathrm{A}}$ & $\rho^{A}$ & $\mathrm{r}^{\mathrm{S}}$ & $\rho^{S}$ \\
\hline CSO & 0,02 & 0,02 & 20,0 & 20,0 & 0,0167 & 0,0167 & -1 & -1 \\
\hline CS1 & 0,023 & 0,023 & 21,0 & 21,0 & 0,0177 & 0,0177 & -1 & -1 \\
\hline CS6 & 0,026 & 0,026 & 23,0 & 23,0 & 0,0157 & 0,0157 & -1 & -1 \\
\hline CS7 & 0,021 & 0,021 & 24,0 & 24,0 & 0,0167 & 0,0167 & -1 & -1 \\
\hline CS3 & 0,01 & 0,01 & 30,0 & 30,0 & 0,0187 & 0,0187 & -1 & -1 \\
\hline CS4 & 0,012 & 0,012 & 31,0 & 31,0 & 0,0147 & 0,0147 & -1 & -1 \\
\hline CS8 & 0,015 & 0,015 & 33,0 & 33,0 & 0,0167 & 0,0167 & -1 & -1 \\
\hline CS9 & 0,013 & 0,013 & 30,0 & 30,0 & 0,0167 & 0,0167 & -1 & -1 \\
\hline LA0 & 0,053 & 10,6 & 4,3 & -1 & 1,6 & -1 & -1 & -1 \\
\hline LA1 & 0,05 & 10,0 & 4,0 & -1 & 1,5 & -1 & -1 & -1 \\
\hline LA2 & 0,051 & 10,2 & 4,5 & -1 & 1,4 & -1 & -1 & -1 \\
\hline IAO & 0,005 & 0,005 & 0,0167 & 0,0167 & 0,0167 & 0,0167 & -1 & -1 \\
\hline IA4 & 0,005 & 0,005 & 3,0 & 3,0 & 0,0167 & 0,0167 & -1 & -1 \\
\hline IA7 & 0,02 & 0,02 & -1 & -1 & 0,0157 & 0,0167 & 1,0 & 1,0 \\
\hline AS0 & 0,02 & 0,02 & -1 & -1 & 0,0167 & -1 & 1,0 & -1 \\
\hline AS7 & 0,025 & 0,025 & -1 & -1 & 0,0167 & 0,0167 & 0,5 & -1 \\
\hline RF0 & 0,005 & 0,005 & 1,0 & 1,0 & 0,0167 & 0,0167 & -1 & -1 \\
\hline XSO & 0,02 & 0,02 & 3,0 & -1 & 0,0167 & -1 & -1 & -1 \\
\hline SVO & 0,02 & 0,02 & -1 & -1 & 0,25 & 0,25 & 1,0 & 1,0 \\
\hline SV3 & 0,025 & 0,025 & -1 & -1 & 0,0167 & 0,0167 & 1,2 & 1,2 \\
\hline SV5 & 0,02 & 0,02 & -1 & -1 & 0,25 & -1 & 1,0 & -1 \\
\hline SV7 & 0,028 & 0,028 & -1 & -1 & 0,0167 & 0,0167 & 1,1 & -1 \\
\hline IS0 & 0,005 & 0,005 & 3,0 & 3,0 & 0,0167 & 0,0167 & -1 & -1 \\
\hline IS3 & 0,0053 & 0,0053 & 3,2 & 3,2 & 0,0147 & 0,0167 & -1 & -1 \\
\hline IS5 & 0,022 & 0,022 & -1 & -1 & 0,0157 & -1 & 1,1 & -1 \\
\hline IS7 & 0,02 & 0,02 & -1 & -1 & 0,0177 & 0,0167 & 1,0 & -1 \\
\hline FU0 & 0,02 & 0,02 & 0,5 & -1 & -1 & -1 & -1 & -1 \\
\hline RC0 & 0,022 & 0,022 & -1 & -1 & 0,0177 & 3 & 1,0 & -1 \\
\hline RC5 & 0,02 & 0,02 & -1 & -1 & 0,0157 & 0,0167 & 1,3 & -1 \\
\hline TF0 & 0,007 & 0,007 & 5,0 & 5,0 & -1 & -1 & -1 & -1 \\
\hline TF5 & 0,07 & 0,07 & 5,0 & -1 & -1 & -1 & -1 & -1 \\
\hline SA0 & 0,0 & 0,0 & -1 & -1 & -1 & -1 & -1 & -1 \\
\hline
\end{tabular}

Tabla A2-3. Datos numéricos base de los parámetros de fiabilidad de componentes individuales.

Debe recordarse que las duraciones medias de la reposición de servicio de los elementos que aparecen en la Tabla A2-3, se refieren al tiempo medio de reparación propiamente dicho y que, para obtener el tiempo medio de reposición total, hay que sumarle a aquél el tiempo medio de desplazamiento y localización que será introducido de forma interactiva para cada experimento de simulación que efectuemos, tal como se indicó en el apartado 2.3. 


\section{DATOS TOPOLÓGICOS DE LAS REDES}

\section{ANEXO 3: DATOS TOPOLÓGICOS DE LAS REDES}

En este anexo describiremos la estructura y contenidos de los ficheros topológicos que definen la estructura de las redes aérea, subterránea y mixta utilizadas en esta Tesis.

Los datos topológicos de las redes definidas en el apartado 3.1 son los que a continuación se detallan.

\section{- Características generales de la red.}

1. Número de componentes total en la red.

2. Número de puntos de carga total en la red.

3. Potencia instalada, en kVA.

4. Potencia media demandable, en kVA.

5. Duración media del buen tiempo $(\mathrm{N})$, en horas.

6. Duración media del mal tiempo (S), en horas.

7. Tensión nominal, en voltios.

8. Tensión en subestación, en voltios.

9. Máxima caída de tensión admisible en condiciones de emergencia. En valores p.u., tomando como base la tensión en la subestación. 
- Características de los puntos de carga.

1. Número de orden del punto carga o centro de transformación expresado en el esquema topológico.

2. Número del nudo de carga que corresponde al centro de transformación. A partir de este número seremos capaces de localizar el punto carga en los esquemas topológicos representados en las figuras 3.1, 3.2 y 3.3 del apartado 3.1 .

3. Número de máquinas (transformadores) en el centro de tranformación.

4. Potencia de cada máquina en kVA.

5. $\mathrm{N}^{\mathrm{o}}$ de clientes por máquina.

6. $\operatorname{Cos} \varphi$ o factor de potencia de la carga.

7. Factor de Carga, considerado como el cociente entre la potencia media demandada y la instalada.

\section{- Características de los elementos.}

1. Nombre: la estructura del nombre será una cadena de la forma LA 1 0001. Las dos primeras letras indican el tipo de elemento y el primer número indica el subtipo (según la nomenclatura del Anexo 1). La última cifra indica el número de orden del elemento expresado en el esquema topológico que podemos ver en las figuras 3.1, 3.2 y 3.3 del apartado 3.1.

2. $1^{\circ}$ nudo al comienzo del elemento en el esquema topológico.

3. $2^{\circ}$ nudo al final del elemento en el esquema topológico.

4. Estado: representa el estado del componente, en principio abierto "0" y cerrado "1". El "2" representa el estado fuera de servicio.

5. Longitud en km. de las líneas aéreas y cables subterráneos. En el resto de los elementos aparecerá un cero. 
RED SUBTERRÁNEA

\begin{tabular}{|ll|}
\hline \multicolumn{2}{|c|}{ CARACTERISTICAS GENERALES DE LA RED } \\
\hline Número de componentes & 123 \\
Número de puntos de carga & 29 \\
\hline Potencia instalada & $11.340 \mathrm{kVA}$ \\
\hline Potencia media demandable & $8.321 \mathrm{kVA}$ \\
\hline Tensión nominal & $15000,0 \mathrm{~V}$ \\
\hline Tensión en subestación & $15200,0 \mathrm{~V}$ \\
\hline Máxima caida de tensión admisible & 0,1 \\
\hline
\end{tabular}

\begin{tabular}{|c|c|c|c|c|c|c|}
\hline \multicolumn{7}{|c|}{ CARACTERISTICAS DE LOS PUNTOS DE CARGA } \\
\hline $\begin{array}{l}\mathrm{N}^{0} \mathrm{de} \\
\text { orden }\end{array}$ & $\begin{array}{c}\mathrm{N}^{\mathbf{o}} \text { de } \\
\text { nudo de } \\
\text { carga }\end{array}$ & $\begin{array}{c}\mathrm{N}^{\mathbf{0}} \mathrm{de} \\
\text { máquinas }\end{array}$ & $\begin{array}{c}\text { Potencia por } \\
\text { máquina } \\
\text { (KVA) }\end{array}$ & $\begin{array}{l}\mathrm{N}^{\circ} \text { de clientes } \\
\text { por máquina }\end{array}$ & $\operatorname{Cos} \phi$ & $\begin{array}{c}\text { Factor de } \\
\text { Carga }\end{array}$ \\
\hline 0 & 7 & 1 & 630,0 & 100 & 0,8 & 0,8 \\
\hline 1 & 9 & 1 & 630,0 & 100 & 0,8 & 0,8 \\
\hline 2 & 14 & 1 & 250,0 & 100 & 0,8 & 0,8 \\
\hline 3 & 16 & 1 & 250,0 & 100 & 0,8 & 0,8 \\
\hline 4 & 21 & 1 & 250,0 & 100 & 0,8 & 0,8 \\
\hline 5 & 23 & 1 & 630,0 & 100 & 0,8 & 0,7 \\
\hline 6 & 34 & 1 & 250,0 & 100 & 0,8 & 0,8 \\
\hline 7 & 36 & 1 & 250,0 & 100 & 0,8 & 0,8 \\
\hline 8 & 41 & 1 & 250,0 & 100 & 0,8 & 0,7 \\
\hline 9 & 43 & 1 & 250,0 & 100 & 0,8 & 0,8 \\
\hline 10 & 48 & 1 & 400,0 & 100 & 0,8 & 0,8 \\
\hline 11 & 53 & 1 & 630,0 & 100 & 0,8 & 0,4 \\
\hline 12 & 55 & 1 & 250,0 & 100 & 0,8 & 0,8 \\
\hline 13 & 63 & 1 & 250,0 & 100 & 0,8 & 0,6 \\
\hline 14 & 65 & 1 & 400,0 & 100 & 0,8 & 0,7 \\
\hline 15 & 70 & 1 & 250,0 & 100 & 0,8 & 0,8 \\
\hline 16 & 72 & 1 & 400,0 & 100 & 0,8 & 0,8 \\
\hline 17 & 77 & 1 & 630,0 & 100 & 0,8 & 0,8 \\
\hline 18 & 83 & 1 & 400,0 & 100 & 0,8 & 0,8 \\
\hline 19 & 85 & 1 & 630,0 & 100 & 0,8 & 0,6 \\
\hline 20 & 90 & 1 & 400,0 & 100 & 0,8 & 0,5 \\
\hline 21 & 96 & 1 & 400,0 & 100 & 0,8 & 0,8 \\
\hline 22 & 98 & 1 & 250,0 & 100 & 0,8 & 0,8 \\
\hline 23 & 103 & 1 & 250,0 & 100 & 0,8 & 0,6 \\
\hline 24 & 108 & 1 & 250,0 & 100 & 0,8 & 0,8 \\
\hline 25 & 110 & 1 & 400,0 & 100 & 0,8 & 0,8 \\
\hline 26 & 116 & 1 & 250,0 & 100 & 0,8 & 0,7 \\
\hline 27 & 118 & 1 & 630,0 & 100 & 0,8 & 0,8 \\
\hline 28 & 123 & 1 & 630,0 & 100 & 0,8 & 0,8 \\
\hline
\end{tabular}




\begin{tabular}{|c|c|c|c|c|}
\hline \multicolumn{5}{|c|}{$\begin{array}{c}\text { CARACTERÍSTICAS DE LOS } \\
\text { ELEMENTOS }\end{array}$} \\
\hline Nombre & $\begin{array}{c}\mathbf{1}^{\circ} \\
\text { Nudo } \\
\end{array}$ & $\begin{array}{c}2^{\circ} \\
\text { Nudo }\end{array}$ & Estado & $\begin{array}{l}\text { Longitu } \\
\mathbf{d}(\mathbf{K m})\end{array}$ \\
\hline CS00001 & 3 & 4 & 1 & 0,7 \\
\hline CS00002 & 10 & 11 & 1 & 0,6 \\
\hline $\mathrm{CS} 00003$ & 17 & 18 & 1 & 1,1 \\
\hline CS00004 & 24 & 25 & 1 & 0,5 \\
\hline CS00005 & 30 & 31 & 1 & 0,2 \\
\hline CS00006 & 37 & 38 & 1 & 0,2 \\
\hline CS00007 & 44 & 45 & 1 & 0,3 \\
\hline CS00008 & 49 & 50 & 1 & 0,4 \\
\hline CS00009 & 56 & 57 & 1 & 0,2 \\
\hline CS00010 & 59 & 60 & 1 & 0,2 \\
\hline CS00011 & 66 & 67 & 1 & 0,2 \\
\hline CS00012 & 73 & 74 & 1 & 0,3 \\
\hline CS00013 & 79 & 80 & 1 & 0,8 \\
\hline CS00014 & 86 & 87 & 1 & 0,3 \\
\hline CS00015 & 91 & 92 & 1 & 0,4 \\
\hline CS00016 & 92 & 93 & 1 & 0,2 \\
\hline $\mathrm{CS} 00017$ & 99 & 100 & 1 & 0,1 \\
\hline CS00018 & 104 & 105 & 1 & 0,1 \\
\hline CS00019 & 111 & 112 & 2 & 0,2 \\
\hline CSO0020 & 92 & 113 & 1 & 0,4 \\
\hline CS00021 & 119 & 120 & 1 & 0,2 \\
\hline SA00001 & 1 & 2 & 1 & 0,0 \\
\hline IA00001 & 2 & 3 & 1 & 0,0 \\
\hline IA40002 & 25 & 26 & 1 & 0,0 \\
\hline IA40003 & 28 & 29 & 1 & 0,0 \\
\hline IA40004 & 57 & 58 & 1 & 0,0 \\
\hline IA40005 & 58 & 79 & 1 & 0,0 \\
\hline SV00001 & 26 & 27 & 1 & 0,0 \\
\hline SV00002 & 27 & 28 & 1 & 0,0 \\
\hline SV00003 & 29 & 30 & 1 & 0,0 \\
\hline SV00004 & 58 & 59 & 1 & 0,0 \\
\hline IS00001 & 4 & 5 & 1 & 0,0 \\
\hline RF00001 & 5 & 6 & 1 & 0,0 \\
\hline TF00001 & 6 & 7 & 1 & 0,0 \\
\hline IS00101 & 5 & 10 & 1 & 0,0 \\
\hline RF00101 & 5 & 8 & 1 & 0,0 \\
\hline TF00101 & 8 & 9 & 1 & 0,0 \\
\hline IS00002 & 11 & 12 & 1 & 0,0 \\
\hline RF00002 & 12 & 13 & 1 & 0,0 \\
\hline TF00002 & 13 & 14 & 1 & 0,0 \\
\hline IS00102 & 12 & 17 & 1 & 0,0 \\
\hline RF00102 & 12 & 15 & 1 & 0,0 \\
\hline TF00102 & 15 & 16 & 1 & 0,0 \\
\hline IS00003 & 18 & 19 & 1 & 0,0 \\
\hline RF00003 & 19 & 20 & 1 & 0,0 \\
\hline TF00003 & 20 & 21 & 1 & 0,0 \\
\hline IS00103 & 19 & 24 & 1 & 0,0 \\
\hline RF00103 & 19 & 22 & 1 & 0,0 \\
\hline TF00103 & 22 & 23 & 1 & 0,0 \\
\hline IS00004 & 31 & 32 & 1 & 0,0 \\
\hline RF00004 & 32 & 33 & 1 & 0,0 \\
\hline TF00004 & 33 & 34 & 1 & 0,0 \\
\hline IS00104 & 32 & 37 & 1 & 0,0 \\
\hline RF00104 & 32 & 35 & 1 & 0,0 \\
\hline TF00104 & 35 & 36 & 1 & 0,0 \\
\hline IS00005 & 38 & 39 & 1 & 0,0 \\
\hline RF00005 & 39 & 40 & 1 & 0,0 \\
\hline TF00005 & 40 & 41 & 1 & 0,0 \\
\hline IS00105 & 39 & 44 & 1 & 0,0 \\
\hline RF00105 & 39 & 42 & 1 & 0,0 \\
\hline TF00105 & 42 & 43 & 1 & 0,0 \\
\hline IS00006 & 45 & 46 & 1 & 0,0 \\
\hline
\end{tabular}

\begin{tabular}{|c|c|c|c|c|}
\hline \multicolumn{5}{|c|}{$\begin{array}{c}\text { CARACTERÍSTICAS DE LOS } \\
\text { ELEMENTOS }\end{array}$} \\
\hline Nombre & $\begin{array}{c}1^{\circ} \\
\text { Nudo }\end{array}$ & $\begin{array}{c}2^{\circ} \\
\text { Nudo }\end{array}$ & Estado & $\begin{array}{l}\text { Longitu } \\
\text { d (Km) }\end{array}$ \\
\hline RF00006 & 46 & 47 & 1 & 0,0 \\
\hline TF00006 & 47 & 48 & 1 & 0,0 \\
\hline IS00106 & 46 & 49 & 1 & 0,0 \\
\hline IS00007 & 50 & 51 & 1 & 0,0 \\
\hline RF00007 & 51 & 52 & 1 & 0,0 \\
\hline TF00007 & 52 & 53 & 1 & 0,0 \\
\hline IS00107 & 51 & 56 & 1 & 0,0 \\
\hline RF00107 & 51 & 54 & 1 & 0,0 \\
\hline TF00107 & 54 & 55 & 1 & 0,0 \\
\hline IS00008 & 60 & 61 & 1 & 0,0 \\
\hline RF00008 & 61 & 62 & 1 & 0,0 \\
\hline TF00008 & 62 & 63 & 1 & 0,0 \\
\hline IS00108 & 61 & 66 & 1 & 0,0 \\
\hline RF00108 & 61 & 64 & 1 & 0,0 \\
\hline TF00108 & 64 & 65 & 1 & 0,0 \\
\hline IS00009 & 67 & 68 & 1 & 0,0 \\
\hline RF00009 & 68 & 69 & 1 & 0,0 \\
\hline TF00009 & 69 & 70 & 1 & 0,0 \\
\hline IS00109 & 68 & 73 & 1 & 0,0 \\
\hline RF00109 & 68 & 71 & 1 & 0,0 \\
\hline TF00109 & 71 & 72 & 1 & 0,0 \\
\hline IS00010 & 74 & 75 & 1 & 0,0 \\
\hline RF00010 & 75 & 76 & 1 & 0,0 \\
\hline TF00010 & 76 & 77 & 1 & 0,0 \\
\hline IS00110 & 75 & 78 & 0 & 0,0 \\
\hline IS00011 & 80 & 81 & 1 & 0,0 \\
\hline RF00011 & 81 & 82 & 1 & 0,0 \\
\hline TF00011 & 82 & 83 & 1 & 0,0 \\
\hline IS00111 & 81 & 86 & 1 & 0,0 \\
\hline RF00111 & 81 & 84 & 1 & 0,0 \\
\hline TF00111 & 84 & 85 & 1 & 0,0 \\
\hline IS00012 & 87 & 88 & 1 & 0,0 \\
\hline RF00012 & 88 & 89 & 1 & 0,0 \\
\hline TF00012 & 89 & 90 & 1 & 0,0 \\
\hline IS00112 & 88 & 91 & 1 & 0,0 \\
\hline IS00013 & 93 & 94 & 1 & 0,0 \\
\hline RF00013 & 94 & 95 & 1 & 0,0 \\
\hline TF00013 & 95 & 96 & 1 & 0,0 \\
\hline IS00113 & 94 & 99 & 1 & 0,0 \\
\hline RF00113 & 94 & 97 & 1 & 0,0 \\
\hline TF00113 & 97 & 98 & 1 & 0,0 \\
\hline IS00014 & 100 & 101 & 1 & 0,0 \\
\hline RF00014 & 101 & 102 & 1 & 0,0 \\
\hline TF00014 & 102 & 103 & 1 & 0,0 \\
\hline IS00114 & 101 & 104 & 1 & 0,0 \\
\hline IS00015 & 105 & 106 & 1 & 0,0 \\
\hline RF00015 & 106 & 107 & 1 & 0,0 \\
\hline TF00015 & 107 & 108 & 1 & 0,0 \\
\hline IS00115 & 106 & 111 & 1 & 0,0 \\
\hline RF00115 & 106 & 109 & 1 & 0,0 \\
\hline TF00115 & 109 & 110 & 1 & 0,0 \\
\hline IS00016 & 113 & 114 & 1 & 0,0 \\
\hline RF00016 & 114 & 115 & 1 & 0,0 \\
\hline TF00016 & 115 & 116 & 1 & 0,0 \\
\hline IS00116 & 114 & 119 & 1 & 0,0 \\
\hline RF00116 & 114 & 117 & 1 & 0,0 \\
\hline TF00116 & 117 & 118 & 1 & 0,0 \\
\hline IS00017 & 120 & 121 & 1 & 0,0 \\
\hline RF00017 & 121 & 122 & 1 & 0,0 \\
\hline TF00017 & 122 & 123 & 1 & 0,0 \\
\hline IS00117 & 121 & 124 & 0 & 0,0 \\
\hline
\end{tabular}


RED AÉREA

\section{CARACTERISTICAS GENERALES DE LA RED}

\begin{tabular}{ll}
\hline Número de componentes & 105
\end{tabular}

$\begin{array}{ll}\text { Número de puntos de carga } & 18\end{array}$

$\begin{array}{ll}\text { Potencia instalada } & 1.680 \mathrm{kVA}\end{array}$

Potencia media demandable $\quad 1.344 \mathrm{kVA}$

Duración media del buen tiempo(N) 400,0 horas

Duración media del mal tiempo(S) 2,0 horas

\begin{tabular}{ll}
\hline Tensión nominal & $15000,0 \mathrm{~V}$
\end{tabular}

\begin{tabular}{ll}
\hline Tensión en subestación & $15200,0 \mathrm{~V}$
\end{tabular}

Máxima caida de tensión admisible 0,1

\begin{tabular}{|c|c|c|c|c|c|c|}
\hline \multicolumn{7}{|c|}{ CARACTERISTICAS DE LOS PUNTOS DE CARGA } \\
\hline $\begin{array}{c}\mathbf{N}^{\mathbf{0}} \text { de } \\
\text { orden }\end{array}$ & $\begin{array}{c}\mathbf{N}^{\mathbf{0}} \text { de } \\
\text { nudo de } \\
\text { carga }\end{array}$ & $\begin{array}{c}\mathbf{N}^{\mathbf{0}} \text { de } \\
\text { máquinas }\end{array}$ & $\begin{array}{c}\text { Potencia por } \\
\text { máquina } \\
\text { KVA) }\end{array}$ & $\begin{array}{c}\mathbf{N}^{\mathbf{0}} \text { de clientes } \\
\text { por máquina }\end{array}$ & Cos $\phi$ & $\begin{array}{c}\text { Factor de } \\
\text { Carga }\end{array}$ \\
\hline 0 & 18 & 1 & 100,0 & 100 & 0,8 & 0,8 \\
\hline 1 & 22 & 1 & 50,0 & 100 & 0,8 & 0,8 \\
\hline 2 & 26 & 1 & 100,0 & 100 & 0,8 & 0,8 \\
\hline 3 & 32 & 1 & 160,0 & 100 & 0,8 & 0,8 \\
\hline 4 & 37 & 1 & 50,0 & 100 & 0,8 & 0,8 \\
\hline 5 & 42 & 1 & 160,0 & 100 & 0,8 & 0,8 \\
\hline 6 & 147 & 1 & 100,0 & 100 & 0,8 & 0,8 \\
\hline 7 & 51 & 1 & 50,0 & 100 & 0,8 & 0,8 \\
\hline 8 & 161 & 1 & 100,0 & 100 & 0,8 & 0,8 \\
\hline 9 & 94 & 1 & 100,0 & 100 & 0,8 & 0,8 \\
\hline 10 & 99 & 1 & 50,0 & 100 & 0,8 & 0,8 \\
\hline 11 & 204 & 1 & 100,0 & 100 & 0,8 & 0,8 \\
\hline 12 & 79 & 1 & 160,0 & 100 & 0,8 & 0,8 \\
\hline 13 & 88 & 1 & 50,0 & 100 & 0,8 & 0,8 \\
\hline 14 & 84 & 1 & 100,0 & 100 & 0,8 & 0,8 \\
\hline 15 & 68 & 1 & 100,0 & 100 & 0,8 & 0,8 \\
\hline 16 & 73 & 1 & 100,0 & 100 & 0,8 & 0,8 \\
\hline 17 & 55 & 1 & 50,0 & 100 & 0,8 & 0,8 \\
\hline
\end{tabular}




\begin{tabular}{|c|c|c|c|c|}
\hline \multicolumn{5}{|c|}{$\begin{array}{c}\text { CARACTERÍSTICAS DE LOS } \\
\text { ELEMENTOS }\end{array}$} \\
\hline Nombre & $\begin{array}{c}1^{\circ} \\
\text { Nudo }\end{array}$ & $\begin{array}{c}2^{\circ} \\
\text { Nudo }\end{array}$ & Estado & $\begin{array}{l}\text { Longitu } \\
\text { d }(\mathbf{K m})\end{array}$ \\
\hline LA10001 & 3 & 4 & 1 & 1,0 \\
\hline LA10002 & 4 & 5 & 1 & 1,0 \\
\hline LA10003 & 5 & 6 & 1 & 1,0 \\
\hline LA10004 & 6 & 7 & 1 & 1,0 \\
\hline LA10005 & 10 & 11 & 1 & 1,0 \\
\hline LA10006 & 11 & 12 & 1 & 1,0 \\
\hline LA10007 & 12 & 13 & 1 & 1,0 \\
\hline LA10008 & 13 & 14 & 2 & 1,0 \\
\hline LA10009 & 23 & 24 & 1 & 0,5 \\
\hline LA10017 & 15 & 16 & 1 & 0,5 \\
\hline LA10018 & 19 & 20 & 1 & 0,5 \\
\hline LA10020 & 27 & 28 & 1 & 1,0 \\
\hline LA10021 & 29 & 30 & 1 & 0,5 \\
\hline LA10022 & 28 & 33 & 1 & 1,0 \\
\hline LA10023 & 34 & 35 & 1 & 0,5 \\
\hline LA10024 & 33 & 38 & 1 & 1,0 \\
\hline LA10025 & 39 & 40 & 1 & 0,5 \\
\hline LA10026 & 38 & 43 & 1 & 1,0 \\
\hline LA10027 & 44 & 45 & 1 & 2,5 \\
\hline LA10051 & 48 & 49 & 1 & 0,5 \\
\hline LA10052 & 52 & 53 & 1 & 0,5 \\
\hline LA10028 & 56 & 57 & 1 & 1,0 \\
\hline LA10029 & 58 & 59 & 1 & 2,5 \\
\hline LA10030 & 57 & 62 & 1 & 1,0 \\
\hline LA10031 & 89 & 90 & 1 & 1,0 \\
\hline LA10032 & 91 & 92 & 1 & 0,5 \\
\hline LA10033 & 90 & 95 & 1 & 1,0 \\
\hline LA10034 & 96 & 97 & 1 & 0,5 \\
\hline LA10035 & 95 & 100 & 1 & 1,0 \\
\hline LA10036 & 101 & 102 & 1 & 2,5 \\
\hline LA10037 & 74 & 75 & 1 & 1,0 \\
\hline LA10038 & 76 & 77 & 1 & 0,5 \\
\hline LA10039 & 75 & 80 & 1 & 1,0 \\
\hline LA10040 & 81 & 82 & 1 & 0,5 \\
\hline LA10041 & 85 & 86 & 1 & 0,5 \\
\hline LA10042 & 63 & 64 & 1 & 1,0 \\
\hline LA10043 & 65 & 66 & 1 & 0,5 \\
\hline LA10044 & 64 & 69 & 1 & 1,0 \\
\hline LA10045 & 70 & 71 & 1 & 0,5 \\
\hline LA10010 & 7 & 8 & 1 & 0,5 \\
\hline SA00001 & 1 & 2 & 1 & 0,0 \\
\hline IA00001 & 2 & 3 & 1 & 0,0 \\
\hline SV50001 & 8 & 10 & 1 & 0,0 \\
\hline SV50002 & 45 & 46 & 1 & 0,0 \\
\hline SV50003 & 59 & 60 & 1 & 0,0 \\
\hline SV50004 & 102 & 103 & 1 & 0,0 \\
\hline XS00001 & 4 & 15 & 1 & 0,0 \\
\hline XS00002 & 5 & 19 & 1 & 0,0 \\
\hline XS00003 & 6 & 23 & 1 & 0,0 \\
\hline XS00004 & 7 & 27 & 1 & 0,0 \\
\hline XS00005 & 28 & 29 & 1 & 0,0 \\
\hline XS00006 & 33 & 34 & 1 & 0,0 \\
\hline XS00007 & 38 & 39 & 1 & 0,0 \\
\hline XS00008 & 43 & 44 & 1 & 0,0 \\
\hline XS00009 & 11 & 48 & 1 & 0,0 \\
\hline XS00010 & 57 & 58 & 1 & 0,0 \\
\hline XS00011 & 90 & 91 & 1 & 0,0 \\
\hline XS00012 & 95 & 96 & 1 & 0,0 \\
\hline XS00013 & 100 & 101 & 1 & 0,0 \\
\hline XS00014 & 75 & 76 & 1 & 0,0 \\
\hline XS00015 & 80 & 85 & 1 & 0,0 \\
\hline XS00016 & 80 & 81 & 1 & 0,0 \\
\hline XS00017 & 64 & 65 & 1 & 0,0 \\
\hline XS00018 & 69 & 70 & 1 & 0,0 \\
\hline XS00019 & 13 & 52 & 1 & 0,0 \\
\hline
\end{tabular}

\begin{tabular}{|c|c|c|c|c|}
\hline \multicolumn{3}{|c|}{$\begin{array}{l}\text { CARACTERÍSTICAS } \\
\text { ELEMENTOS }\end{array}$} & DE & \multirow{2}{*}{$\begin{array}{c}\text { LOS } \\
\text { Longitu } \\
\text { d }(\mathrm{Km})\end{array}$} \\
\hline Nombre & $\begin{array}{c}1^{\mathbf{0}} \\
\text { Nudo }\end{array}$ & $\begin{array}{c}2^{\mathbf{0}} \\
\text { Nudo }\end{array}$ & Estado & \\
\hline AS00001 & 12 & 56 & 1 & 0,0 \\
\hline AS00002 & 62 & 89 & 1 & 0,0 \\
\hline AS00003 & 62 & 74 & 1 & 0,0 \\
\hline AS00004 & 62 & 63 & 1 & 0,0 \\
\hline FU00001 & 16 & 17 & 1 & 0,0 \\
\hline TF50001 & 17 & 18 & 1 & 0,0 \\
\hline FU00002 & 20 & 21 & 1 & 0,0 \\
\hline TF50002 & 21 & 22 & 1 & 0,0 \\
\hline FU00003 & 24 & 25 & 1 & 0,0 \\
\hline TF50003 & 25 & 26 & 1 & 0,0 \\
\hline FU00004 & 30 & 31 & 1 & 0,0 \\
\hline TF50004 & 31 & 32 & 1 & 0,0 \\
\hline FU00005 & 35 & 36 & 1 & 0,0 \\
\hline TF50005 & 36 & 37 & 1 & 0,0 \\
\hline FU00006 & 40 & 41 & 1 & 0,0 \\
\hline TF50006 & 41 & 42 & 1 & 0,0 \\
\hline FU00007 & 46 & 47 & 1 & 0,0 \\
\hline TF50007 & 47 & 147 & 1 & 0,0 \\
\hline FU00008 & 49 & 50 & 1 & 0,0 \\
\hline TF50008 & 50 & 51 & 1 & 0,0 \\
\hline FU00009 & 60 & 61 & 1 & 0,0 \\
\hline TF50009 & 61 & 161 & 1 & 0,0 \\
\hline FU00010 & 92 & 93 & 1 & 0,0 \\
\hline TF50010 & 93 & 94 & 1 & 0,0 \\
\hline FU00011 & 97 & 98 & 1 & 0,0 \\
\hline TF50011 & 98 & 99 & 1 & 0,0 \\
\hline FU00012 & 103 & 104 & 1 & 0,0 \\
\hline TF50012 & 104 & 204 & 1 & 0,0 \\
\hline FU00013 & 77 & 78 & 1 & 0,0 \\
\hline TF50013 & 78 & 79 & 1 & 0,0 \\
\hline FU00014 & 86 & 87 & 1 & 0,0 \\
\hline TF50014 & 87 & 88 & 1 & 0,0 \\
\hline FU00015 & 82 & 83 & 1 & 0,0 \\
\hline TF50015 & 83 & 84 & 1 & 0,0 \\
\hline FU00016 & 66 & 67 & 1 & 0,0 \\
\hline TF50016 & 67 & 68 & 1 & 0,0 \\
\hline FU00017 & 71 & 72 & 1 & 0,0 \\
\hline TF50017 & 72 & 73 & 1 & 0,0 \\
\hline FU00018 & 53 & 54 & 1 & 0,0 \\
\hline TF50018 & 54 & 55 & 1 & 0,0 \\
\hline
\end{tabular}


RED MIXTA

\begin{tabular}{|ll|}
\hline \multicolumn{2}{|c|}{ CARACTERISTICAS GENERALES DE LA RED } \\
\hline Número de componentes & 300 \\
Número de puntos de carga & 59 \\
\hline Potencia instalada & $7.430 \mathrm{kVA}$ \\
\hline Potencia media demandable & $5.629 \mathrm{kVA}$ \\
\hline Duración media del buen tiempo(N) & 400,0 horas \\
\hline Duración media del mal tiempo(S) & 2,0 horas \\
\hline Tensión nominal & $15000,0 \mathrm{~V}$ \\
\hline Tensión en subestación & $15200,0 \mathrm{~V}$ \\
\hline Máxima caida de tensión admisible & 0,1 \\
\hline
\end{tabular}

\begin{tabular}{|c|c|c|c|c|c|c|}
\hline \multicolumn{7}{|l|}{ CARACTERISTICAS DE LOS PUNTOS DE CARGA } \\
\hline $\begin{array}{c}\mathbf{N}^{\mathbf{0}} \text { de } \\
\text { orden }\end{array}$ & $\begin{array}{c}\mathbf{N}^{\mathbf{0}} \text { de } \\
\text { nudo de } \\
\text { carga }\end{array}$ & $\begin{array}{c}\mathbf{N}^{\mathbf{0}} \text { de } \\
\text { máquinas }\end{array}$ & $\begin{array}{c}\text { Potencia por } \\
\text { máquina } \\
\text { (KVA) }\end{array}$ & $\begin{array}{c}\mathbf{N}^{\mathbf{0}} \text { de clientes } \\
\text { por máquina }\end{array}$ & Cos $\phi$ & $\begin{array}{c}\text { Factor de } \\
\text { Carga }\end{array}$ \\
\hline 0 & 18 & 1 & 100,0 & 100 & 0,8 & 0,8 \\
\hline 1 & 22 & 1 & 50,0 & 100 & 0,8 & 0,8 \\
\hline 2 & 32 & 1 & 160,0 & 100 & 0,8 & 0,8 \\
\hline 3 & 37 & 1 & 50,0 & 100 & 0,8 & 0,8 \\
\hline 4 & 42 & 1 & 160,0 & 100 & 0,8 & 0,8 \\
\hline 5 & 48 & 1 & 100,0 & 100 & 0,8 & 0,8 \\
\hline 6 & 52 & 1 & 50,0 & 100 & 0,8 & 0,8 \\
\hline 7 & 63 & 1 & 100,0 & 100 & 0,8 & 0,8 \\
\hline 8 & 96 & 1 & 100,0 & 100 & 0,8 & 0,8 \\
\hline 9 & 101 & 1 & 50,0 & 100 & 0,8 & 0,8 \\
\hline 10 & 1071 & 1 & 100,0 & 100 & 0,8 & 0,8 \\
\hline 11 & 81 & 1 & 160,0 & 100 & 0,8 & 0,8 \\
\hline 12 & 90 & 1 & 50,0 & 100 & 0,8 & 0,8 \\
\hline 13 & 86 & 1 & 100,0 & 100 & 0,8 & 0,8 \\
\hline 14 & 70 & 1 & 100,0 & 100 & 0,8 & 0,8 \\
\hline 15 & 75 & 1 & 100,0 & 100 & 0,8 & 0,8 \\
\hline 16 & 56 & 1 & 50,0 & 100 & 0,8 & 0,8 \\
\hline 17 & 147 & 1 & 100,0 & 100 & 0,8 & 0,8 \\
\hline 18 & 140 & 1 & 100,0 & 100 & 0,8 & 0,8 \\
\hline 19 & 26 & 1 & 50,0 & 100 & 0,8 & 0,8 \\
\hline 20 & 22 & 1 & 100,0 & 100 & 0,8 & 0,8 \\
\hline 21 & 34 & 1 & 100,0 & 100 & 0,8 & 0,8 \\
\hline 22 & 54 & 1 & 50,0 & 100 & 0,8 & 0,8 \\
\hline 23 & 90 & 1 & 50,0 & 100 & 0,8 & 0,8 \\
\hline 24 & 86 & 1 & 100,0 & 100 & 0,8 & 0,8 \\
\hline 25 & 70 & 1 & 50,0 & 100 & 0,8 & 0,8 \\
\hline 26 & 74 & 1 & 100,0 & 100 & 0,8 & 0,8 \\
\hline
\end{tabular}


CARACTERISTICAS DE LOS PUNTOS DE CARGA

\begin{tabular}{|c|c|c|c|c|c|c|}
\hline $\begin{array}{l}\mathrm{N}^{0} \mathrm{de} \\
\text { orden }\end{array}$ & $\begin{array}{c}\mathrm{N}^{\circ} \text { de } \\
\text { nudo de } \\
\text { carga } \\
\end{array}$ & $\begin{array}{c}\mathbf{N}^{0} \text { de } \\
\text { máquinas }\end{array}$ & $\begin{array}{c}\text { Potencia por } \\
\text { máquina } \\
\text { (KVA) }\end{array}$ & $\begin{array}{l}\mathrm{N}^{0} \text { de clientes } \\
\text { por máquina }\end{array}$ & $\operatorname{Cos} \phi$ & $\begin{array}{c}\text { Factor de } \\
\text { Carga }\end{array}$ \\
\hline 27 & 164 & 1 & 100,0 & 100 & 0,8 & 0,8 \\
\hline 28 & 78 & 1 & 100,0 & 100 & 0,8 & 0,8 \\
\hline 29 & 130 & 1 & 50,0 & 100 & 0,8 & 0,8 \\
\hline 30 & 94 & 1 & 250,0 & 100 & 0,8 & 0,8 \\
\hline 31 & 96 & 1 & 250,0 & 100 & 0,8 & 0,8 \\
\hline 32 & 01 & 1 & 100,0 & 100 & 0,8 & 0,8 \\
\hline 33 & 03 & 1 & 100,0 & 100 & 0,8 & 0,8 \\
\hline 34 & 208 & 1 & 100,0 & 100 & 0,8 & 0,8 \\
\hline 35 & 210 & 1 & 250,0 & 100 & 0,8 & 0,7 \\
\hline 36 & 221 & 1 & 100,0 & 100 & 0,8 & 0,8 \\
\hline 37 & 223 & 1 & 100,0 & 100 & 0,8 & 0,8 \\
\hline 38 & 228 & 1 & 100,0 & 100 & 0,8 & 0,7 \\
\hline 39 & 230 & 1 & 100,0 & 100 & 0,8 & 0,8 \\
\hline 40 & 235 & 1 & 200,0 & 100 & 0,8 & 0,8 \\
\hline 41 & 240 & 1 & 250,0 & 100 & 0,8 & 0,4 \\
\hline 42 & 242 & 1 & 100,0 & 100 & 0,8 & 0,8 \\
\hline 43 & 250 & 1 & 100,0 & 100 & 0,8 & 0,6 \\
\hline 44 & 252 & 1 & 200,0 & 100 & 0,8 & 0,7 \\
\hline 45 & 257 & 1 & 100,0 & 100 & 0,8 & 0,8 \\
\hline 46 & 259 & 1 & 200,0 & 100 & 0,8 & 0,8 \\
\hline 47 & 264 & 1 & 250,0 & 100 & 0,8 & 0,8 \\
\hline 48 & 270 & 1 & 200,0 & 100 & 0,8 & 0,8 \\
\hline 49 & 272 & 1 & 250,0 & 100 & 0,8 & 0,6 \\
\hline 50 & 277 & 1 & 200,0 & 100 & 0,8 & 0,5 \\
\hline 51 & 283 & 1 & 200,0 & 100 & 0,8 & 0,8 \\
\hline 52 & 285 & 1 & 100,0 & 100 & 0,8 & 0,8 \\
\hline 53 & 290 & 1 & 100,0 & 100 & 0,8 & 0,6 \\
\hline 54 & 295 & 1 & 100,0 & 100 & 0,8 & 0,8 \\
\hline 55 & 297 & 1 & 200,0 & 100 & 0,8 & 0,8 \\
\hline 56 & 303 & 1 & 100,0 & 100 & 0,8 & 0,7 \\
\hline 57 & 305 & 1 & 250,0 & 100 & 0,8 & 0,8 \\
\hline 58 & 310 & 1 & 250,0 & 100 & 0,8 & 0,8 \\
\hline
\end{tabular}




\begin{tabular}{|c|c|c|c|c|}
\hline \multicolumn{5}{|c|}{$\begin{array}{c}\text { CARACTERÍSTICAS DE LOS } \\
\text { ELEMENTOS }\end{array}$} \\
\hline Nombre & $\begin{array}{c}1^{\circ} \\
\text { Nudo }\end{array}$ & $\begin{array}{c}2^{\circ} \\
\text { Nudo }\end{array}$ & Estado & $\begin{array}{l}\text { Longitu } \\
\text { d (Km) }\end{array}$ \\
\hline LA10001 & 3 & 4 & 1 & 1.0 \\
\hline LA10002 & 4 & 5 & 1 & 1.0 \\
\hline LA10003 & 5 & 7 & 1 & 1.0 \\
\hline LA10005 & 10 & 11 & 1 & 1.0 \\
\hline LA10006 & 11 & 12 & 1 & 1.0 \\
\hline LA10007 & 12 & 13 & 1 & 1.0 \\
\hline LA10008 & 13 & 14 & 1 & 1.0 \\
\hline LA10010 & 7 & 8 & 1 & 0.5 \\
\hline LA10017 & 15 & 16 & 1 & 0.5 \\
\hline LA10018 & 19 & 20 & 1 & 0.5 \\
\hline LA10020 & 27 & 28 & 1 & 1.0 \\
\hline LA10021 & 29 & 30 & 1 & 0.5 \\
\hline LA10022 & 28 & 33 & 1 & 1.0 \\
\hline LA10023 & 34 & 35 & 1 & 0.5 \\
\hline LA10024 & 33 & 38 & 1 & 1.0 \\
\hline LA10025 & 39 & 40 & 1 & 0.5 \\
\hline LA10026 & 38 & 43 & 1 & 1.0 \\
\hline LA10027 & 44 & 45 & 1 & 2.5 \\
\hline LA10051 & 49 & 50 & 1 & 0.5 \\
\hline LA10052 & 53 & 54 & 1 & 0.5 \\
\hline LA10028 & 57 & 58 & 1 & 1.0 \\
\hline LA10029 & 59 & 60 & 1 & 2.5 \\
\hline LA10030 & 58 & 64 & 1 & 1.0 \\
\hline LA10031 & 91 & 92 & 1 & 1.0 \\
\hline LA10032 & 93 & 94 & 1 & 0.5 \\
\hline LA10033 & 92 & 97 & 1 & 1.0 \\
\hline LA10034 & 98 & 99 & 1 & 0.5 \\
\hline LA10035 & 97 & 102 & 1 & 1.0 \\
\hline LA10036 & 103 & 104 & 1 & 2.5 \\
\hline LA10037 & 76 & 77 & 1 & 1.0 \\
\hline LA10038 & 78 & 79 & 1 & 0.5 \\
\hline LA10039 & 77 & 82 & 1 & 1.0 \\
\hline LA10040 & 83 & 84 & 1 & 0.5 \\
\hline LA10041 & 87 & 88 & 1 & 0.5 \\
\hline LA10042 & 65 & 66 & 1 & 1.0 \\
\hline LA10043 & 67 & 68 & 1 & 0.5 \\
\hline LA10044 & 66 & 71 & 1 & 1.0 \\
\hline LA10045 & 72 & 73 & 1 & 0.5 \\
\hline SA00001 & 1 & 2 & 1 & 0.0 \\
\hline IA00001 & 2 & 3 & 1 & 0.0 \\
\hline SV50001 & 8 & 10 & 1 & 0.0 \\
\hline SV50002 & 45 & 46 & 1 & 0.0 \\
\hline SV50003 & 60 & 61 & 1 & 0.0 \\
\hline SV50004 & 104 & 105 & 1 & 0.0 \\
\hline XS00001 & 4 & 15 & 1 & 0.0 \\
\hline $\mathrm{XS} 00002$ & 5 & 19 & 1 & 0.0 \\
\hline XS00004 & 7 & 27 & 1 & 0.0 \\
\hline XS00005 & 28 & 29 & 1 & 0.0 \\
\hline XS00006 & 33 & 34 & 1 & 0.0 \\
\hline XS00007 & 38 & 39 & 1 & 0.0 \\
\hline XS00008 & 43 & 44 & 1 & 0.0 \\
\hline XS00009 & 11 & 49 & 1 & 0.0 \\
\hline XS00010 & 58 & 59 & 1 & 0.0 \\
\hline XS00011 & 92 & 93 & 1 & 0.0 \\
\hline XS00012 & 97 & 98 & 1 & 0.0 \\
\hline XS00013 & 102 & 103 & 1 & 0.0 \\
\hline XS00014 & 77 & 78 & 1 & 0.0 \\
\hline XS00015 & 82 & 87 & 1 & 0.0 \\
\hline XS00016 & 82 & 83 & 1 & 0.0 \\
\hline XS00017 & 66 & 67 & 1 & 0.0 \\
\hline XS00018 & 71 & 72 & 1 & 0.0 \\
\hline XS00019 & 13 & 53 & 1 & 0.0 \\
\hline
\end{tabular}

\begin{tabular}{|l|c|c|c|c|}
\hline \multicolumn{5}{|c|}{ CARACTERÍSTICAS DE LOS } \\
\hline \multicolumn{2}{|c|}{ ELEMENTOS } \\
\hline Nombre & $\begin{array}{c}\mathbf{1}^{\mathbf{0}} \\
\text { Nudo }\end{array}$ & $\begin{array}{c}\mathbf{2}^{\mathbf{o}} \\
\text { Nudo }^{2}\end{array}$ & Estado & $\begin{array}{c}\text { Longitu } \\
\text { d (Km) }\end{array}$ \\
\hline AS00001 & 12 & 57 & 1 & 0.0 \\
\hline AS00002 & 64 & 91 & 1 & 0.0 \\
\hline AS00003 & 64 & 76 & 1 & 0.0 \\
\hline AS00004 & 64 & 65 & 1 & 0.0 \\
\hline FU00001 & 16 & 17 & 1 & 0.0 \\
\hline TF50001 & 17 & 18 & 1 & 0.0 \\
\hline FU00002 & 20 & 21 & 1 & 0.0 \\
\hline TF50002 & 21 & 22 & 1 & 0.0 \\
\hline FU00004 & 30 & 31 & 1 & 0.0 \\
\hline TF50004 & 31 & 32 & 1 & 0.0 \\
\hline FU00005 & 35 & 36 & 1 & 0.0 \\
\hline TF50005 & 36 & 37 & 1 & 0.0 \\
\hline FU00006 & 40 & 41 & 1 & 0.0 \\
\hline TF50006 & 41 & 42 & 1 & 0.0 \\
\hline FU00007 & 46 & 47 & 1 & 0.0 \\
\hline TF50007 & 47 & 48 & 1 & 0.0 \\
\hline FU00008 & 50 & 51 & 1 & 0.0 \\
\hline TF50008 & 51 & 52 & 1 & 0.0 \\
\hline FU00009 & 61 & 62 & 1 & 0.0 \\
\hline TF50009 & 62 & 63 & 1 & 0.0 \\
\hline FU00010 & 94 & 95 & 1 & 0.0 \\
\hline TF50010 & 95 & 96 & 1 & 0.0 \\
\hline FU00011 & 99 & 100 & 1 & 0.0 \\
\hline TF50011 & 100 & 101 & 1 & 0.0 \\
\hline FU00012 & 105 & 106 & 1 & 0.0 \\
\hline TF50012 & 106 & 107 & 1 & 0.0 \\
\hline FU00013 & 79 & 80 & 1 & 0.0 \\
\hline TF50013 & 80 & 81 & 1 & 0.0 \\
\hline FU00014 & 88 & 89 & 1 & 0.0 \\
\hline TF50014 & 89 & 90 & 1 & 0.0 \\
\hline FU00015 & 84 & 85 & 1 & 0.0 \\
\hline TF50015 & 85 & 86 & 1 & 0.0 \\
\hline FU00016 & 68 & 69 & 1 & 0.0 \\
\hline TF50016 & 69 & 70 & 1 & 0.0 \\
\hline FU00017 & 73 & 74 & 1 & 0.0 \\
\hline TF50017 & 74 & 75 & 1 & 0.0 \\
\hline FU00018 & 54 & 55 & 1 & 0.0 \\
\hline TF50018 & 55 & 56 & 1 & 0.0 \\
\hline LA10203 & 108 & 109 & 1 & 1.0 \\
\hline LA10204 & 109 & 110 & 1 & 1.0 \\
\hline LA10205 & 112 & 113 & 1 & 1.0 \\
\hline LA10206 & 113 & 114 & 1 & 1.0 \\
\hline LA10207 & 114 & 115 & 1 & 1.0 \\
\hline LA10209 & 144 & 145 & 1 & 0.5 \\
\hline LA10210 & 110 & 111 & 1 & 0.5 \\
\hline LA10220 & 135 & 136 & 1 & 1.0 \\
\hline LA10223 & 137 & 138 & 1 & 0.5 \\
\hline LA10224 & 115 & 116 & 1 & 1.0 \\
\hline LA10225 & 123 & 124 & 1 & 0.5 \\
\hline LA10226 & 116 & 117 & 1 & 1.0 \\
\hline LA10227 & 118 & 119 & 1 & 2.5 \\
\hline LA10251 & 131 & 132 & 1 & 0.5 \\
\hline LA10252 & 127 & 128 & 1 & 0.5 \\
\hline LA10228 & 148 & 149 & 1 & 1.0 \\
\hline LA10229 & 150 & 151 & 1 & 2.5 \\
\hline LA10230 & 149 & 155 & 1 & 1.0 \\
\hline LA10231 & 179 & 180 & 1 & 1.0 \\
\hline LA10234 & 187 & 188 & 1 & 0.5 \\
\hline LA10235 & 180 & 181 & 1 & 1.0 \\
\hline LA10237 & 182 & 183 & 1 & 2.5 \\
\hline
\end{tabular}




\begin{tabular}{|c|c|c|c|c|}
\hline \multicolumn{5}{|c|}{$\begin{array}{c}\text { CARACTERÍSTICAS DE LOS } \\
\text { ELEMENTOS }\end{array}$} \\
\hline Nombre & $\begin{array}{c}1^{\circ} \\
\text { Nudo }\end{array}$ & $\begin{array}{c}2^{\circ} \\
\text { Nudo }\end{array}$ & Estado & $\begin{array}{l}\text { Longitu } \\
\text { d (Km) }\end{array}$ \\
\hline LA10241 & 167 & 168 & 1 & 0.5 \\
\hline LA10242 & 156 & 157 & 1 & 1.0 \\
\hline LA10243 & 161 & 162 & 1 & 0.5 \\
\hline LA10244 & 157 & 158 & 1 & 1.0 \\
\hline LA10245 & 175 & 176 & 1 & 0.5 \\
\hline IA00002 & 2 & 108 & 1 & 0.0 \\
\hline SV50201 & 111 & 112 & 1 & 0.0 \\
\hline SV50202 & 119 & 120 & 1 & 0.0 \\
\hline SV50203 & 151 & 152 & 1 & 0.0 \\
\hline SV50204 & 183 & 184 & 1 & 0.0 \\
\hline XS00203 & 109 & 144 & 1 & 0.0 \\
\hline XS00204 & 110 & 135 & 1 & 0.0 \\
\hline XS00206 & 136 & 137 & 1 & 0.0 \\
\hline XS00207 & 116 & 123 & 1 & 0.0 \\
\hline XS00208 & 117 & 118 & 1 & 0.0 \\
\hline XS00209 & 113 & 131 & 1 & 0.0 \\
\hline XS00210 & 149 & 150 & 1 & 0.0 \\
\hline XS00212 & 180 & 187 & 1 & 0.0 \\
\hline XS00213 & 181 & 182 & 1 & 0.0 \\
\hline XS00215 & 166 & 167 & 1 & 0.0 \\
\hline XS00216 & 166 & 171 & 1 & 0.0 \\
\hline XS00217 & 157 & 161 & 1 & 0.0 \\
\hline XS00218 & 158 & 175 & 1 & 0.0 \\
\hline XS00219 & 115 & 127 & 1 & 0.0 \\
\hline AS00201 & 114 & 148 & 1 & 0.0 \\
\hline AS00202 & 155 & 179 & 1 & 0.0 \\
\hline AS00203 & 155 & 165 & 1 & 0.0 \\
\hline AS00204 & 155 & 156 & 1 & 0.0 \\
\hline FU00201 & 145 & 146 & 1 & 0.0 \\
\hline TF50201 & 146 & 147 & 1 & 0.0 \\
\hline FU00202 & 138 & 139 & 1 & 0.0 \\
\hline TF50202 & 139 & 140 & 1 & 0.0 \\
\hline FU00204 & 124 & 125 & 1 & 0.0 \\
\hline TF50204 & 125 & 126 & 1 & 0.0 \\
\hline FU00205 & 120 & 121 & 1 & 0.0 \\
\hline TF50205 & 121 & 122 & 1 & 0.0 \\
\hline FU00203 & 132 & 133 & 1 & 0.0 \\
\hline TF50203 & 133 & 134 & 1 & 0.0 \\
\hline FU00206 & 152 & 153 & 1 & 0.0 \\
\hline TF50206 & 153 & 154 & 1 & 0.0 \\
\hline FU00207 & 188 & 189 & 1 & 0.0 \\
\hline TF50207 & 189 & 190 & 1 & 0.0 \\
\hline FU00208 & 184 & 185 & 1 & 0.0 \\
\hline TF50208 & 185 & 186 & 1 & 0.0 \\
\hline FU00209 & 168 & 169 & 1 & 0.0 \\
\hline TF50209 & 169 & 170 & 1 & 0.0 \\
\hline FU00210 & 172 & 173 & 1 & 0.0 \\
\hline TF50210 & 173 & 174 & 1 & 0.0 \\
\hline FU00211 & 162 & 163 & 1 & 0.0 \\
\hline TF50211 & 163 & 164 & 1 & 0.0 \\
\hline FU00212 & 176 & 177 & 1 & 0.0 \\
\hline TF50212 & 177 & 178 & 1 & 0.0 \\
\hline FU00213 & 128 & 129 & 1 & 0.0 \\
\hline TF50213 & 129 & 130 & 1 & 0.0 \\
\hline CS00301 & 26 & 191 & 1 & 0.5 \\
\hline CS00302 & 197 & 198 & 1 & 0.4 \\
\hline CS00303 & 204 & 205 & 1 & 0.9 \\
\hline CS00304 & 211 & 212 & 1 & 0.4 \\
\hline CS00305 & 217 & 218 & 1 & 0.2 \\
\hline CS00306 & 224 & 225 & 1 & 0.2 \\
\hline CS00307 & 231 & 232 & 1 & 0.3 \\
\hline CS00308 & 236 & 237 & 1 & 0.4 \\
\hline CS00309 & 243 & 244 & 1 & 0.2 \\
\hline
\end{tabular}

\section{CARACTERÍSTICAS DE LOS} ELEMENTOS

\begin{tabular}{|c|c|c|c|c|}
\hline Nombre & $\begin{array}{c}1^{\circ} \\
\text { Nudo }\end{array}$ & $\begin{array}{c}2^{\circ} \\
\text { Nudo }\end{array}$ & Estado & $\begin{array}{c}\text { Longitu } \\
\text { d }(\mathrm{Km})\end{array}$ \\
\hline CS00310 & 246 & 247 & 1 & 0.2 \\
\hline CS00311 & 253 & 254 & 1 & 0.2 \\
\hline CS00312 & 260 & 261 & 1 & 0.3 \\
\hline CS00313 & 266 & 267 & 1 & 0.6 \\
\hline CS00314 & 273 & 274 & 1 & 0.3 \\
\hline CS00315 & 278 & 279 & 1 & 0.4 \\
\hline CS00316 & 279 & 280 & 1 & 0.2 \\
\hline CS00317 & 286 & 287 & 1 & 0.1 \\
\hline CS00318 & 291 & 292 & 1 & 0.1 \\
\hline CS00319 & 298 & 299 & 2 & 0.2 \\
\hline CSO0320 & 279 & 300 & 1 & 0.4 \\
\hline CS00321 & 306 & 307 & 1 & 0.2 \\
\hline IA00301 & 14 & 26 & 1 & 0.0 \\
\hline IA40302 & 212 & 213 & 1 & 0.0 \\
\hline IA40303 & 215 & 216 & 1 & 0.0 \\
\hline IA40304 & 244 & 245 & 1 & 0.0 \\
\hline IA40305 & 245 & 266 & 1 & 0.0 \\
\hline SV00301 & 213 & 214 & 1 & 0.0 \\
\hline SV00302 & 214 & 215 & 1 & 0.0 \\
\hline SV00303 & 216 & 217 & 1 & 0.0 \\
\hline SV00304 & 245 & 246 & 1 & 0.0 \\
\hline IS00301 & 191 & 192 & 1 & 0.0 \\
\hline RF00301 & 192 & 193 & 1 & 0.0 \\
\hline TF00301 & 193 & 194 & 1 & 0.0 \\
\hline IS00401 & 192 & 197 & 1 & 0.0 \\
\hline RF00401 & 192 & 195 & 1 & 0.0 \\
\hline TF00401 & 195 & 196 & 1 & 0.0 \\
\hline IS00302 & 198 & 199 & 1 & 0.0 \\
\hline RF00302 & 199 & 200 & 1 & 0.0 \\
\hline TF00302 & 200 & 201 & 1 & 0.0 \\
\hline IS00402 & 199 & 204 & 1 & 0.0 \\
\hline RF00402 & 199 & 202 & 1 & 0.0 \\
\hline TF00402 & 202 & 203 & 1 & 0.0 \\
\hline IS00303 & 205 & 206 & 1 & 0.0 \\
\hline RF00303 & 206 & 207 & 1 & 0.0 \\
\hline TF00303 & 207 & 208 & 1 & 0.0 \\
\hline IS00403 & 206 & 211 & 1 & 0.0 \\
\hline RF00403 & 206 & 209 & 1 & 0.0 \\
\hline TF00403 & 209 & 210 & 1 & 0.0 \\
\hline IS00304 & 218 & 219 & 1 & 0.0 \\
\hline RF00304 & 219 & 220 & 1 & 0.0 \\
\hline TF00304 & 220 & 221 & 1 & 0.0 \\
\hline IS00404 & 219 & 224 & 1 & 0.0 \\
\hline RF00404 & 219 & 222 & 1 & 0.0 \\
\hline TF00404 & 222 & 223 & 1 & 0.0 \\
\hline IS00305 & 225 & 226 & 1 & 0.0 \\
\hline RF00305 & 226 & 227 & 1 & 0.0 \\
\hline TF00305 & 227 & 228 & 1 & 0.0 \\
\hline IS00405 & 226 & 231 & 1 & 0.0 \\
\hline RF00405 & 226 & 229 & 1 & 0.0 \\
\hline TF00405 & 229 & 230 & 1 & 0.0 \\
\hline IS00306 & 232 & 233 & 1 & 0.0 \\
\hline RF00306 & 233 & 234 & 1 & 0.0 \\
\hline TF00306 & 234 & 235 & 1 & 0.0 \\
\hline IS00406 & 233 & 236 & 1 & 0.0 \\
\hline IS00307 & 237 & 238 & 1 & 0.0 \\
\hline RF00307 & 238 & 239 & 1 & 0.0 \\
\hline TF00307 & 239 & 240 & 1 & 0.0 \\
\hline IS00407 & 238 & 243 & 1 & 0.0 \\
\hline RF00407 & 238 & 241 & 1 & 0.0 \\
\hline TF00407 & 241 & 242 & 1 & 0.0 \\
\hline IS00308 & 247 & 248 & 1 & 0.0 \\
\hline RF00308 & 248 & 249 & 1 & 0.0 \\
\hline
\end{tabular}

\begin{tabular}{|l|c|c|c|c|}
\hline \multicolumn{5}{|c}{ CARACTERÍSTICAS DE LOS } \\
\hline Nombre & $\begin{array}{c}\mathbf{1}^{\mathbf{0}} \\
\text { Nudo }\end{array}$ & $\begin{array}{c}\mathbf{2}^{\mathbf{0}} \\
\text { Nudo }\end{array}$ & Estado & $\begin{array}{c}\text { Longitu } \\
\text { d (Km) }\end{array}$ \\
\hline
\end{tabular}




$$
\text { 业 }
$$


